\title{
Gefügebildung und Verdichtungsvorgänge bei weichmagnetischen Ferriten
}

\author{
Dissertation \\ zur Erlangung des Doktorgrades \\ der Mathematisch-Naturwissenschaftlichen Fakultäten \\ der Georg-August-Universität zu Göttingen
}

vorgelegt von

Jörg Dreikorn

aus Freiburg im Breisgau

Göttingen 2005 
D7

Referent: Prof. Dr. H.C. Freyhardt

Korreferent: Prof. Dr. W. Felsch

Tag der mündlichen Prüfung: 08. November 2005 


\section{Inhaltsverzeichnis}

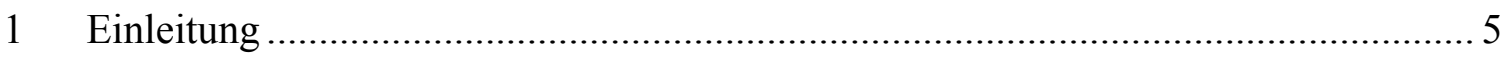

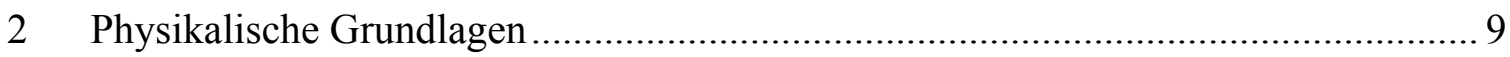

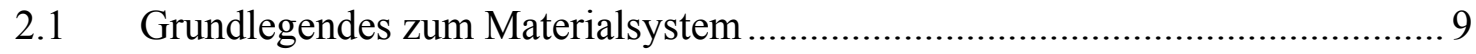

2.1.1 Kristallstruktur und Magnetismus ........................................................ 9

2.1.2 Magnetokristalline Anisotropieenergie............................................... 12

2.1.3 Magnetoelastische Energie................................................................. 15

2.1.4 Empirischer Ansatz zur Bestimmung der Anfangspermeabilität.............. 16

2.1.5 Magnetische Domänen und Magnetisierungsprozess .............................. 17

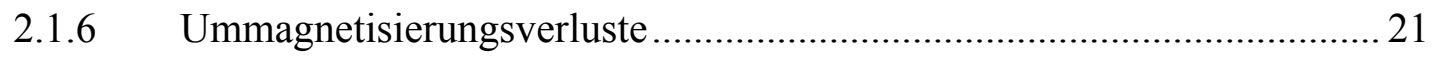

2.1.7 Mikrostruktur und magnetische Eigenschaften.................................... 21

2.2 Gefügebildungs- und Kornwachstumsprozesse …...................................... 23

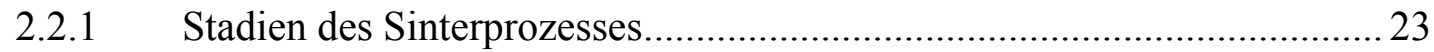

2.2.2 Diskontinuierliches Kornwachstum ...................................................... 29

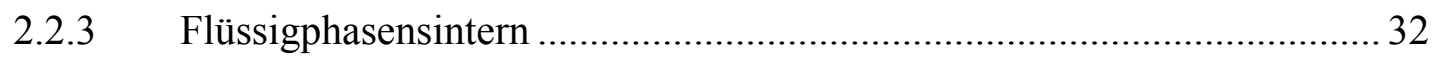

2.2.4 Wismutoxid und Molybdäntrioxid als Dotierungen ............................... 33

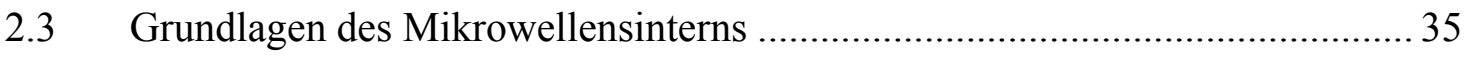

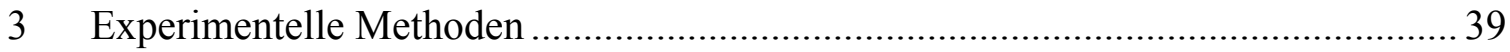

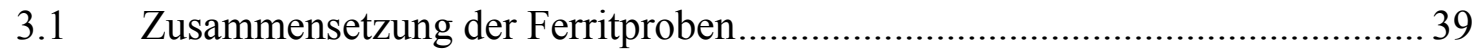

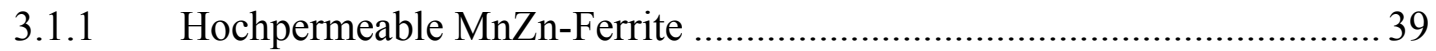

3.1.2 Ferrit für die Leistungsübertragung und NiZn-Ferrit ............................. 41

3.1.3 Überprüfung der Zusammensetzung mittels RFA .................................. 42

3.2 Herstellung von weichmagnetischen Ferriten .............................................. 42

3.2.1 Pulverpräparation und Pressen von Grünkörpern ................................... 43

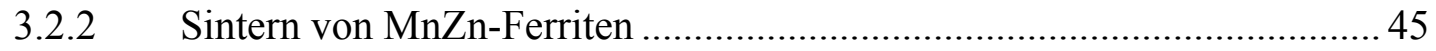

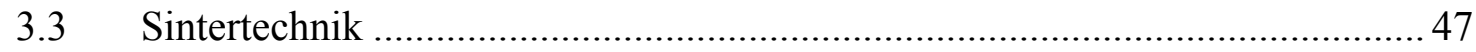

3.3.1 Laborofen mit Widerstandsbeheizung ..................................................... 47

3.3.2 Elektro - Mikrowellen - Hybridsintertechnik ........................................... 48

3.4 Messung der magnetischen Eigenschaften................................................. 50

3.4.1 Messung der komplexen Permeabilität .................................................. 50

3.4.2 Messung der Ummagnetisierungsverluste ............................................. 51

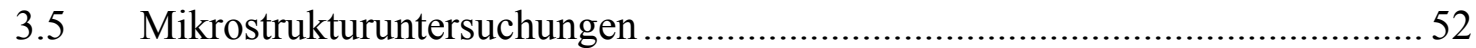


3.5.1 Licht- und Rasterelektronenmikroskopie _.............................................52

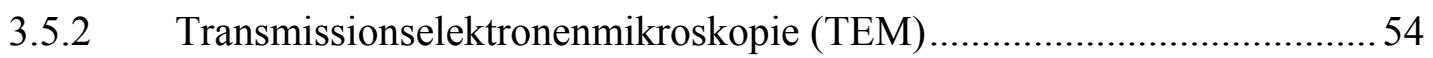

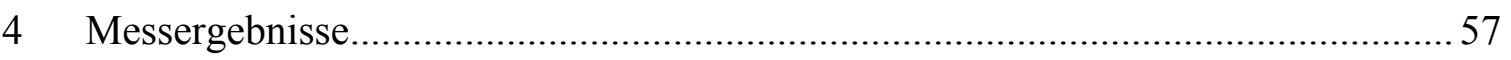

4.1 Einfluss schmelzfließender Phasen auf die Eigenschaften von MnZn-Ferriten 57

4.1.1 Untersuchungen zur Wismutoxid-Ferrit-Phasengrenze an Presslingen .... 57

4.1.2 Mikrostruktur bei Ferriten mit Wismutoxiddotierung ............................ 59

4.1.3 Mikrostruktur bei Molybdäntrioxiddotierung ......................................... 66

4.1.4 Transmissionselektronenmikroskopische Untersuchungen (TEM) .......... 70

4.1.5 Röntgenfluoreszenzanalyse (RFA) ................................................... 74

4.1.6 Messung der magnetischen Eigenschaften.......................................... 75

4.1.7 Messung weiterer magnetischer Eigenschaften ................................... 77

4.2 Gefügeoptimierung durch Elektro - Mikrowellen - Hybridsintern .................... 79

4.2.1 Vergleich mit konventioneller Sinterung ............................................. 79

4.2.2 Optimierung des Mikrowellenanteils bei NiZn-Ferriten........................... 82

4.2.3 Versuche mit MnZn-Ferriten für die Leistungsübertragung ..................... 84

4.2.4 TEM-Untersuchung der Korngrenzen................................................... 86

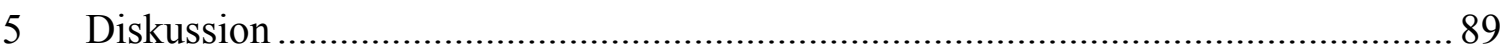

5.1 Wirkung von $\mathrm{Bi}_{2} \mathrm{O}_{3}$ und $\mathrm{MoO}_{3}$ als schmelzfließende Zusätze......................... 89

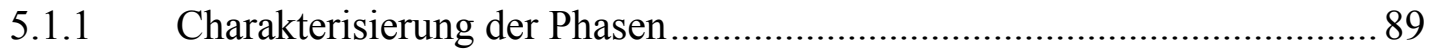

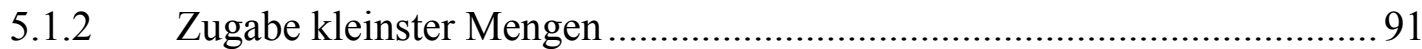

5.1.3 Entstehung von diskontinuierlichem Kornwachstum ............................. 94

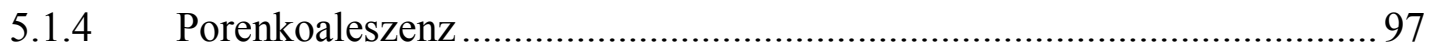

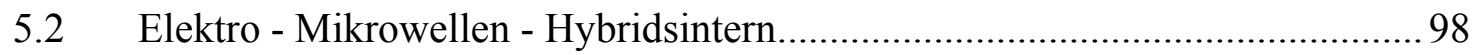

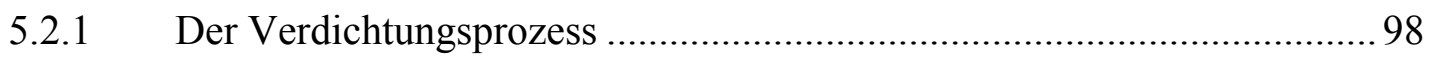

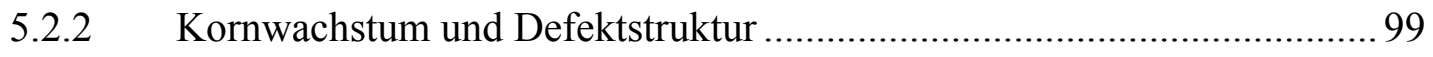

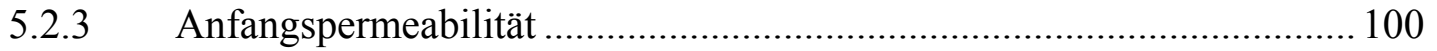

5.2.4 Der optimale Anteil an Mikrowellenleistung......................................... 102

5.2.5 Verlustabsenkung bei Leistungsferriten.............................................. 103

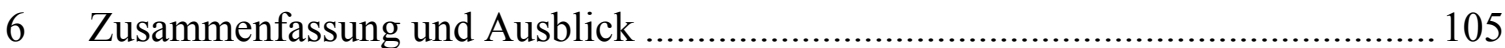

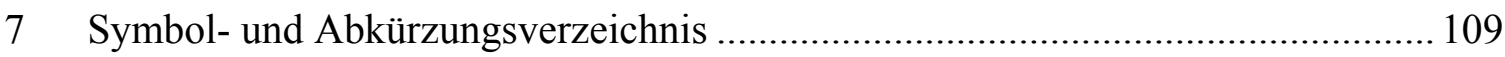

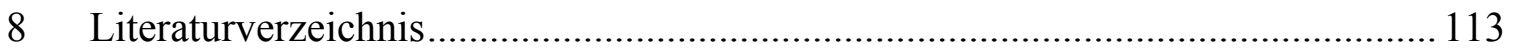




\section{Einleitung}

Gemischte Metalloxide mit $\mathrm{Fe}^{3+}$ als kationischer Hauptkomponente werden als Ferrite bezeichnet. Abhängig von der auftretenden Kristallstruktur und Zusammensetzung zeigen diese Systeme hart- oder weichmagnetische Eigenschaften. Grundlegende Arbeiten hierzu gehen auf L. Néel [1] und S. Hilpert [2, 3] zurück und wurden zum Teil schon Anfang des zwanzigsten Jahrhunderts durchgeführt. Erste technische Anwendungen ergaben sich mit der Entwicklung von Kupfer-Zink-Ferriten $\left(\mathrm{Ni}_{\mathrm{x}} \mathrm{Cu}_{1-\mathrm{x}} \mathrm{Fe}_{2} \mathrm{O}_{4}{ }^{[\mathrm{i}]}\right)$ durch Y. Tako und T. Takei in Japan (1932) und deren patentrechtlichen Verwertung durch die TDK-Corporation ab 1937 [4, 5].

In Europa führten u. a. E.J.W. Verwey und E.L. Heilmann Untersuchungen an Ferriten mit Spinellstruktur durch und klärten die Verteilung der Metallionen auf den Gitterplätzen auf [6]. Auf dieser Grundlage entwickelte L. Néel seine Theorie des unkompensierten Antiferromagnetismus und bezeichnete das Phänomen als Ferrimagnetismus [7]. Arbeiten, die bei Philips seit Mitte der Dreißigerjahre u.a. von Snoek [8, 9] sowie von Smit und Wijn [10] durchgeführt wurden, führten zur breiten Anwendung von weichmagnetischen gemischten Nickel-Zink- und Mangan-ZinkFerriten $\left(\mathrm{Ni}_{\mathrm{x}} \mathrm{Zn}_{1-\mathrm{x}-} \mathrm{Fe}_{2} \mathrm{O}_{4}\right.$ bzw. $\left.\mathrm{Mn}_{\mathrm{x}} \mathrm{Zn}_{1-\mathrm{x}-} \mathrm{Fe}_{2} \mathrm{O}_{4}{ }^{[\mathrm{i}]}\right)$ in Elektronik und Telekommunikation. Vorteilhaft gegenüber den bis dahin eingesetzten metallischen Magnetwerkstoffen ist insbesondere der verhältnismäßig hohe spezifische Widerstand, der bei den halbleitenden MnZn-Ferriten typischerweise bei ca. 1 - $10 \Omega \mathrm{m}$ und bei den isolierenden NiZn-Ferriten bei $10^{5}-10^{7} \Omega \mathrm{m}$ liegt. Er ist damit um mehrere Größenordnungen höher als bei kristallinen metallischen Magnetwerkstoffen $(0,1-1 \mu \Omega m)$ und begünstigt die Verwendung von Ferriten bei Frequenzen $>1 \mathrm{kHz}$, bei denen die in Metallen auftretenden hohen Wirbelstromverluste den bei den Ferriten vorhandenen Nachteil der vergleichsweise niedrigen Permeabilität und Sättigung ausgleichen ${ }^{[i]}$.

Das Einsatzfeld der MnZn- und NiZn-Ferrite im Bereich der Hochfrequenztechnik ist breit gestreut und man kann die verwendeten Materialien in Untergruppen unterteilen.

[i] Genauer ist die Strukturformel $\mathrm{Ni}_{\mathrm{x}} \mathrm{Cu}_{1-\mathrm{x}-\mathrm{y}} \mathrm{Fe}_{2+\mathrm{y}} \mathrm{O}_{4+\delta}$, welche die meistens vorhandenen Abweichungen im Eisen- und Sauerstoffgehalt berücksichtigt (y, $\delta<<1)$. Entsprechendes gilt auch für MnZn- und NiZnFerrite.

[ii] Seit ca. 1970 gelingt durch den Einsatz der sogenannten Melt-Spin-Technik die Herstellung dünner amorpher bzw. nanokristalliner magnetischer Metallbänder mit guten magnetischen Eigenschaften und einem verhältnismäßig hohen spezifischen Widerstand $(\rho>1 \mu \Omega m)$. Durch diese Entwicklung erschloss sich auch den metallischen Werkstoffen ein Einsatzfeld bei Frequenzen $>10 \mathrm{kHz}$. 
MnZn-Ferrite mit mittleren und hohen Anfangspermeabilitäten $\left(\mu_{i}=4000-12000\right)$ kommen bei verhältnismäßig niedrigen Aussteuerungen für die Signalübertragung und zur Entstörung bei Frequenzen bis zu $30 \mathrm{MHz}$ zur Anwendung.

Eine zweite Untergruppe der MnZn-Ferrite zeichnet sich durch Sättigungsmagnetisierungen von ca. $500 \mathrm{mT}$ und niedrige Ummagnetisierungsverluste aus. Sie werden auch als Leistungsferrite bezeichnet und dienen der induktiven Leistungsübertragung in einem typischen Frequenzbereich von $20 \mathrm{kHz}$ bis $1 \mathrm{MHz}$ z.B. in Schaltnetzteilen und beim Hochfrequenzschweißen, sowie der Energiespeicherung in Drosselspulen.

Die Gruppe der NiZn - Ferrite, mit ihrem hohen spezifischen Widerstand, wird z.B. für Antennenstäbe bei Frequenzen bis zu $100 \mathrm{MHz}$, für die Unterdrückung hochfrequenter Störströme und Störstrahlungen sowie für Hochspannungsanwendungen eingesetzt.

Bei allen Materialgruppen finden bis in jüngste Zeit Bemühungen zur Verbesserung der elektromagnetischen und mechanischen Eigenschaften statt. Maßgeblich hierfür ist ein anhaltender Trend zur Miniaturisierung der Bauteile und damit verbunden das Anwachsen der Arbeitsfrequenzen, sowie die Erschließung neuer Einsatzfelder mit erhöhten mechanischen und thermischen Anforderungen z.B. in Luft- und Raumfahrt und der Automobilindustrie. Der Fokus der Materialentwicklung liegt insbesondere auf der Optimierung der Mikrostruktur (mikrostrukturelles Konstruieren), wobei der Einfluss von Fremdatomen, Fehlstellen und anderen Gitterdefekten, die Korn- und Porenstruktur, sowie in Korngrenzen und Tripelpunkten segregierte Phasen von Bedeutung sind.

Die vorliegende Arbeit greift diese Bemühungen auf. Sie wurde durch die Forschungsund Entwicklungstätigkeit der Kaschke KG GmbH \& Co, einem Hersteller von Weichferriten angeregt und gemeinsam mit dem Institut für Materialphysik der Universität Göttingen durchgeführt. Des Weiteren werden Ergebnisse aus einem Forschungsprojekt zum Thema Mikrowellen-Hybridsintertechnik aufgegriffen (AiF.-Nr. 12072 B/2), das von der Forschungsgemeinschaft Industrieofenbau (FOGI) initiiert und vom $B M W i$ unterstützt wurde.

Inhalt ist die Optimierung der Mikrostruktur bei MnZn- und NiZn-Ferriten unter Berücksichtigung zweier Aspekte. Zum ersten ist dies das Flüssigphasensintern von MnZn-Ferriten, bei dem es durch Zugabe geringer Mengen an niedrigschmelzenden Oxiden während des Sintervorgangs zum Entstehen einer Schmelze kommt, die den Verdichtungs- und Kornwachstumsprozess beeinflusst. Ziel ist die Erhöhung der Anfangspermeabilität $\mu_{i}$ und damit ein verbesserter Einsatz für Schnittstellenübertrager in 
der Telekommunikation. Zum zweiten sind es die Möglichkeiten, die der Einsatz der Elektro-Mikrowellen-Hybridsintertechnik bei der Herstellung von MnZn- und NiZnFerriten bietet.

Die Arbeit gliedert sich in sechs Kapitel. Das folgenden Kapitel 2 gibt einen Überblick über die physikalischen Grundlagen. Neben den allgemeinen magnetischen Eigenschaften des Materialsystems wird dabei auf die beim Sintern ablaufenden Prozesse und auf die Wechselwirkung des Ferrits mit der Mikrowellenstrahlung eingegangen. In Kapitel 3 erfolgt eine Einführung in die angewandten experimentellen Methoden. Insbesondere werden die Präparation der Proben, die verwendete Messtechnik und die bei den Mikrostrukturuntersuchungen angewandten Verfahren beschrieben. Kapitel 4 ist zweigeteilt und stellt in Abschnitt 4.1 die Ergebnisse zum Flüssigphasensintern und in Abschnitt 4.2 die mittels Hybridsintertechnik erzielten Resultate vor. In Kapitel 5 findet eine Diskussion dieser Ergebnisse statt, während Kapitel 6 das Erreichte zusammenfasst und mit einem Ausblick auf weitere Entwicklungen verbindet. 


\section{Physikalische Grundlagen}

\subsection{Grundlegendes zum Materialsystem}

Bei Chikazumi [11] findet sich eine Einführung in die Physik des Magnetismus, die verstärkt auf oxidische Werkstoffe eingeht, neben der schon erwähnten Monographie von Smit und Wijn [10] existieren weitere Abhandlungen [12, 13], welche sich speziell mit dem Magnetismus der Ferrite beschäftigen.

\subsubsection{Kristallstruktur und Magnetismus}

Die Spinellstruktur

Eine Definition der Ferrite wurde bereits im vorigen Kapitel gegeben. Kristallografisch treten Ferrite mit Perowskit-, Spinell-, Granat- und hexagonaler-Struktur auf (Tabelle 2.1).

\begin{tabular}{l|l|l|l}
\hline Typ & Struktur & Allgemeine Formel $^{\text {ii] }}$ & Anwendung \\
\hline Spinell & kubisch & $\mathrm{M}^{\mathrm{II}} \mathrm{Fe}_{2} \mathrm{O}_{4}$ & weichmagnetische Werkstoffe \\
\hline Granat & kubisch & $\mathrm{M}^{\mathrm{III}}{ }_{3} \mathrm{Fe}_{5} \mathrm{O}_{12}$ & Mikrowellenanwendungen \\
\hline $\begin{array}{l}\text { Magneto- } \\
\text { plumbit }\end{array}$ & hexagonal & $\mathrm{M}^{\mathrm{II}} \mathrm{Fe}_{12} \mathrm{O}_{19}$ & Hartmagnete \\
\hline Perowskit & kubisch & $\mathrm{SE}^{\mathrm{III}}{ }_{1-\mathrm{x}} \mathrm{EA}^{\mathrm{II}}{ }_{\mathrm{x}} \mathrm{MO}_{3}$ & $\begin{array}{l}\text { für M = Mn (Mangante): magneto- } \\
\text { resistive Materialien }\end{array}$ \\
\hline Tabelle 2.1 & \multicolumn{2}{|c|}{$\begin{array}{l}\text { Übersicht über Ferrite und ihre wichtigsten industriellen Einsatzfelder. (M = } \\
\text { Übergangsmetall, SE = Seltenerdmetall, EA = Erdalkalimetall). }\end{array}$}
\end{tabular}

Für diese Arbeit ist nur die Gruppe der weichmagnetischen Ferrite mit Spinellstruktur von Interesse und hierbei insbesondere die Mangan-Zink-Ferrite und die Nickel-ZinkFerrite.

Der Spinell besteht aus einem kubisch-flächenzentrierten $(\mathrm{kfz})$ Sauerstoffanionengitter, in dem sich die Kationen auf die Zwischengitterplätze verteilen. Eine stöchiometrische Einheitszelle enthält 32 Sauerstoffanionen, 8 Metallkationen mit vierfacher Koordination und 16 Metallkationen mit sechsfacher Koordination (Abbildung 2.1). Die Ersteren werden auch als Tetraeder- oder A-Plätze, die Letzteren als Oktaeder- oder B-Plätze

[i] Bei allen angegebenen Ferriten kann es sowohl bzgl. des Eisen- als auch des Sauerstoffgehaltes Abweichungen von der angegebenen Stöchiometrie geben (vgl. Fußnote [i] auf Seite 5). 
bezeichnet. Die chemische Formel ist $\mathrm{AB}_{2} \mathrm{O}_{4}$, wobei sich im Falle eines normalen (2,3)Spinells ${ }^{[\mathrm{i}]}$ das zweiwertige Ion $\mathrm{A}^{2+}$ auf den Tetraederplätzen und das dreiwertige Ion $\mathrm{B}^{3+}$ auf den Oktaederplätzen befindet.

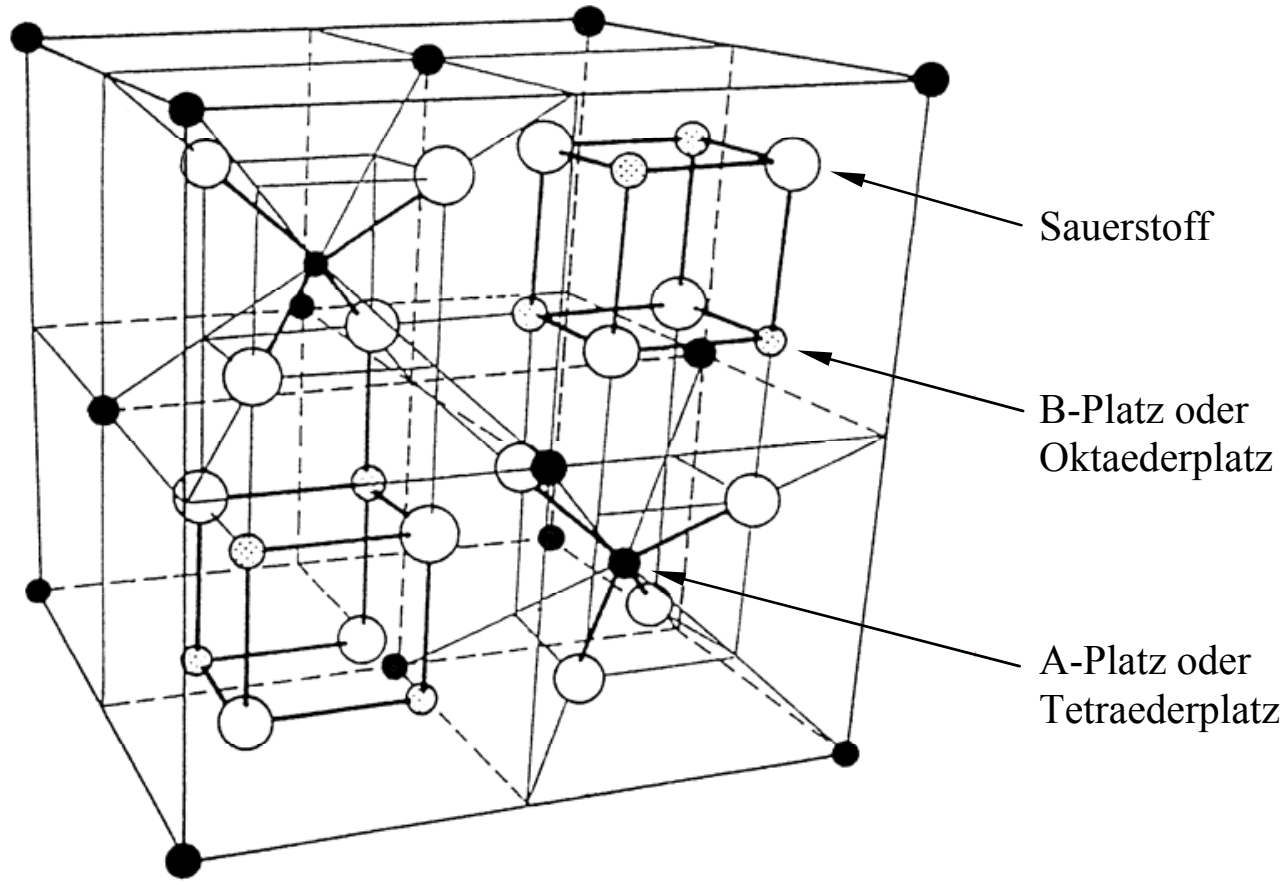

Abbildung 2.1 Einheitszelle des Spinells mit Oktaeder- und Tetraederplätzen nach [14].

Bei den MnZn- und NiZn-Ferriten tritt, wie bei vielen anderen Ferriten auch, teilweise Inversion auf. Dies bedeutet, dass sich sowohl zwei- als auch dreiwertige Kationen auf beiden Zwischengitterplätzen befinden. Mit Hilfe der Mößbauerspektroskopie lassen sich genaue Aussagen über die Besetzung der Untergitterplätze durch die einzelnen Kationen sowie über deren Valenzen machen. Die Ergebnisse sind in Tabelle 2.2 zusammengefasst. Man beachte, dass sowohl Eisen als auch Mangan in ihrer zwei- und dreiwertigen Ionisationsstufe vorkommen.

\begin{tabular}{l|l}
\hline Zwischengitterplatz & Kationen \\
\hline A-Platz oder Tetraederlücke & $\mathrm{Zn}^{2+}, \mathrm{Mn}^{2+}, \mathrm{Mn}^{3+}, \mathrm{Fe}^{3+}$ \\
\hline B-Platz oder Oktaederlücke & $\mathrm{Ni}^{2+}, \mathrm{Mn}^{2+}, \mathrm{Mn}^{3+}, \mathrm{Fe}^{2+}, \mathrm{Fe}^{3+}, \mathrm{Co}^{2+}$ \\
\hline
\end{tabular}

Tabelle 2.2 Verteilung der Ionen bei MnZn- und NiZn-Ferriten.

[i] $(2,3)$ gibt die Wertigkeit der Ionen an. 


\section{Superaustausch-Wechselwirkung}

Die Elektronenkonfigurationen der Übergangsmetalle mit ihrem hohen Anteil an unkompensierten Spinmomenten in der 3d-Schale sind für den bei MnZn-Ferriten beobachteten Magnetismus verantwortlich. Die magnetischen Spinmomente betragen für $\mathrm{Fe}^{3+}$ und $\mathrm{Mn}^{2+}$ jeweils $5 \mu_{B}{ }^{[\mathrm{i}]}$, für $\mathrm{Fe}^{2+}$ und $\mathrm{Mn}^{3+}$ jeweils $4 \mu_{B}$ und für $\mathrm{Zn}^{2+} 0 \mu_{B}$.

Nach dem Kramer-Anderson-Modell [12] erfolgt die Kopplung dieser Momente indirekt antiferromagnetisch über die zwischen den Kationen befindlichen Sauerstoffanionen und wird als Superaustausch-Wechelwirkung bezeichnet. Die Stärke der Kopplung ist umso größer, je geringer der Abstand der Ionen voneinander ist und je weniger ihr Winkel von $180^{\circ}$ abweicht. Im Spinell sind diese Bedingungen am besten für die in Abbildung 2.2 gezeigte Konstellation zwischen einem A- und einem B-Platz -Kation mit einem Kopplungswinkel von $125^{\circ}$ erfüllt. Tritt diese A-B-Kopplung auf ${ }^{[i]}$, so ist sie die energetisch günstigste und man kann zeigen, dass sich zwei antiferromagnetische Untergitter ausbilden, welche mit den kristallografischen A- und B-Plätzen identisch sind.
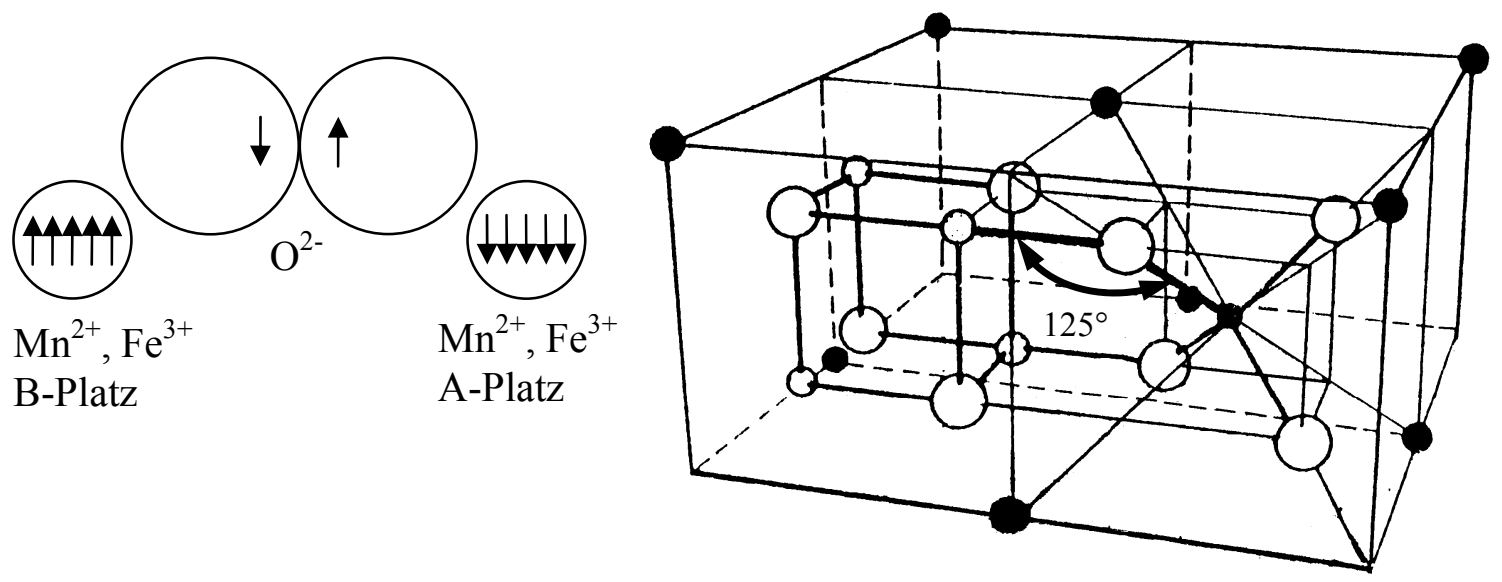

Abbildung 2.2 Darstellung der Superaustauschwechselwirkung A-O-B Kopplung mit einem Winkel von $125^{\circ}$.

Ferrimagnetismus, wie er bei den MnZn- und NiZn-Ferriten beobachtet wird, tritt auf, wenn sich die magnetischen Momente $M_{A}$ und $M_{B}$ der beiden Untergitter nicht vollständig kompensieren.

[i] $\mu_{B}=$ Bohrsches Magneton $\mu_{B}=\hbar e /\left(2 m_{e}\right)=9,274 \cdot 10^{-24} \mathrm{~J} / T$

[ii] Das Auftreten der A-B-Kopplung ist nicht immer gegeben. Bei $\mathrm{Zn}$-Ferrit $\mathrm{ZnFe}_{2} \mathrm{O}_{4}$ besetzt das unmagnetische Zn-Ion sämtliche A-Plätze. Es findet keine A-B-Kopplung, sondern nur B-B-Kopplung statt, was zur Aufspaltung der B-Plätze in zwei antiferromagnetische Untergitter $\mathrm{B}_{1}$ und $\mathrm{B}_{2}$ führt. Die Spins der $\mathrm{Fe}^{3+}$-Ionen auf diesen Untergittern kompensieren sich vollständig. 
Die Sättigungsmagnetisierung ergibt sich angenähert als Differenz der Magnetisierung der Untergitter:

$$
M_{S} \approx\left|M_{B}-M_{A}\right|
$$

\subsubsection{Magnetokristalline Anisotropieenergie}

Die magnetokristalline Anisotropieenergie, die häufig auch verkürzend als Kristallanisotropie bezeichnet wird, spiegelt die Spin-Bahn-Kopplung und die Wechselwirkung der Orbitale mit der Kristallstruktur wieder. Zu ihrer Beschreibung verwendet man die Richtungskosinusse $\alpha_{1}, \alpha_{2}$ und $\alpha_{3}$ zwischen Magnetisierung und den Hauptkristallachsen. Die volumenbezogene Kristallanisotropieenergie $E_{K} / V$ lässt sich für kubische Kristalle in einer Reihe bezüglich der $\alpha_{i}$ entwickeln:

$$
\frac{E_{K}}{V}=K_{0}+K_{1}\left(\alpha_{1}^{2} \alpha_{2}^{2}+\alpha_{2}^{2} \alpha_{3}^{2}+\alpha_{1}^{2} \alpha_{3}^{2}\right)+K_{2}\left(\alpha_{1}^{2} \alpha_{2}^{2} \alpha_{3}^{2}\right)+\ldots
$$

$K_{i}$ ist hierbei die Kristallanisotropiekonstante i-ter Ordnung. Gleichung 2.2 beschreibt die kristallografischen Vorzugsrichtungen der Magnetisierung in Abhängigkeit von den Anisotropiekonstanten (vgl. Abbildung 2.3 b). Von Interesse sind insbesondere die Übergänge zwischen zwei Vorzugsrichtungen, da diese zu einer Zunahme der magnetischen Isotropie und damit auch der Permeabilität führen (vgl. 2.1.4).

a)

$$
\begin{aligned}
& <100>: \frac{E_{K}}{V}=K_{0} \\
& <110>: \frac{E_{K}}{V}=K_{0}+\frac{1}{4} K_{1} \\
& <111>: \frac{E_{K}}{V}=K_{0}+\frac{1}{3} K_{1}+\frac{1}{27} K_{2}
\end{aligned}
$$

b)

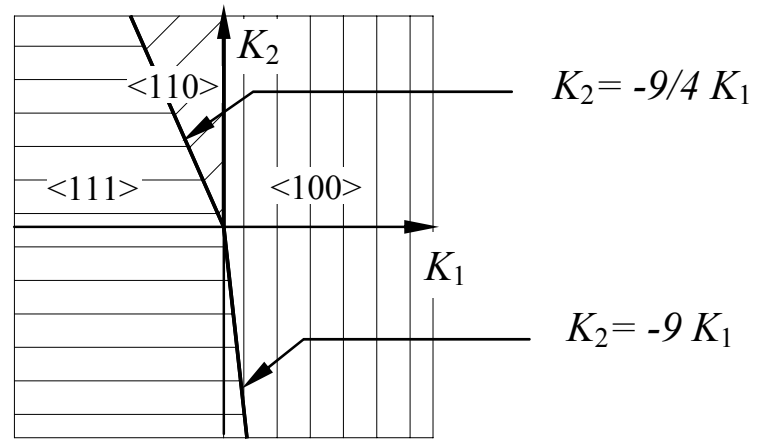

Abbildung 2.3 Aus Gleichung 2.2 ergibt sich die magnetokristalline Anisotropieenergie für die einzelnen kristallografischen Richtungen a). Die Vorzugsrichtungen und die dazugehörigen Übergangsbedingungen b) entsprechen dem Minimum der Anisotropieenergie.

Besonders stark ist der Einfluss der zweiwertigen Ionen $\mathrm{Fe}^{2+}$ und $\mathrm{Co}^{2+}$ auf die Anisotropie. Er lässt sich mit dem sogenannten Einionenmodell der Anisotropie beschreiben, das erstmals von Slonczewski auf kobaltsubstituierten Magnetit angewandt wurde [15] und die Anisotropie als Superposition der Beiträge der einzelnen Kationen angibt. 
Die entstehende Vorzugsrichtung der Magnetisierung für ein einzelnes Ion ergibt sich aus der Kopplung des Spins $S$ an den Bahndrehimpuls $L$ ( $L-S$ - oder Russel-SaundersKopplung), wobei für den Bahndrehimpuls das umgebende Kristallfeld berücksichtigt werden muss, welches aufgrund elektrostatischer Wechselwirkung bei den Kationen zu einer Aufhebung der d-Orbital-Entartung führt. Die dadurch entstehende Vorzugsrichtung für den Bahndrehimpuls ist für die starke magnetokristalline Anisotropie der $\mathrm{Co}^{2+}$ und $\mathrm{Fe}^{2+}$ - Ionen verantwortlich.

Im Rahmen der quantenmechanischen Störungsrechnung wird die Wirkung des Bahndrehimpulses als Störglied im Hamiltonoperator behandelt:

$$
\hat{H}=2 \mu_{B} H \cdot S+\lambda L \cdot S .
$$

Der erste Term beschreibt die Superaustauschwechselwirkung in Form der Wechselwirkung eines effektiven Austauschfeldes (Molekularfeld) $H$ mit dem Spin $S$, während $\lambda L \cdot S$ den Einfluss der Spin-Bahn-Kopplung repräsentiert.

Aus Gleichung 2.3 lässt sich die freie Energie eines einzelnen Ions in Abhängigkeit von der Magnetisierungsrichtung und daraus der entsprechende Beitrag $\mathrm{zu} K_{1}$ und $K_{2}$ berechnen. Für die beschriebenen $\mathrm{Fe}^{2+}$ und $\mathrm{Co}^{2+}$-Ionen auf Oktaederplätzen ergibt sich $K_{1} \gg>0$ und $K_{2}<0$. Beide Ionen haben nach Abbildung 2.3 einen Beitrag zur $<100>$ Richtung als Magnetisierung.

Auch Ionen, welche keinen oder einen eingefrorenen Bahndrehimpuls besitzen, leisten einen Beitrag zur Anisotropieenergie, dieser ist allerdings niedriger als bei den Ionen mit entartetem Grundzustand. Um ihn zu berechnen müssen Glieder höherer Ordnung des Hamilton Operators

$$
\hat{H}=\mu_{B} H \cdot(L+2 S)+\lambda L \cdot S
$$

berücksichtigt werden [12]. Insgesamt ergeben sich qualitativ die in Tabelle 2.3 angegebenen Anteile der einzelnen Ionen zu der Kristallanisotropiekonstante erster Ordnung.

Das Einionenmodell wird durch Vergleichsmessungen sehr gut bestätigt. Insbesondere lässt sich die Temperaturabhängigkeit der Anfangspermeabilität erklären (vgl. Abschnitt 2.1.4). Man beobachtet bei vielen Ferriten die Bildung von sogenannten sekundären Permeabilitätsmaxima (SPM), die mit einem Nulldurchgang der Anisotropiekonstanten 1. Ordnung $K_{1}$ verbunden sind. Am Nulldurchgang kompensieren sich die Beiträge der unterschiedlichen Ionen. Die Lage des SPMs bzw. die Kompensationstemperatur lässt sich durch eine Änderung des $\mathrm{Fe}^{2+}$ - oder $\mathrm{Co}^{2+}$-Gehaltes verschieben [16]. 


\begin{tabular}{l|c|c|l}
\hline Ion & $\begin{array}{c}\text { Gitter- } \\
\text { platz }\end{array}$ & $\begin{array}{c}\text { Beitrag } \\
\text { zu } K_{1}\end{array}$ & Bemerkungen \\
\hline $\mathrm{Fe}^{3+}$ & $\mathrm{B}$ & $<0$ & $\left|K_{1}\left(F e^{3+}\right)_{B}\right| \approx 3\left|K_{1}\left(F e^{3+}\right)_{A}\right|$ \\
\hline $\mathrm{Fe}^{3+}$ & $\mathrm{A}$ & $>0$ & \\
\hline $\mathrm{Fe}^{2+}$ & $\mathrm{B}$ & $>>0$ & \\
\hline $\mathrm{Mn}^{2+}$ & $\mathrm{A}, \mathrm{B}$ & $\sim 0$ & $\left|K_{1}\left(\mathrm{Mn}^{2+}\right)\right| \approx 1 / 10\left|K_{1}\left(F e^{3+}\right)\right|$ \\
\hline $\mathrm{Mn}^{3+}$ & $\mathrm{B}$ & $>0$ & \\
\hline $\mathrm{Co}^{2+}$ & $\mathrm{B}$ & $>>0$ & \\
\hline $\mathrm{Zn}^{2+}$ & $\mathrm{A}$ & - & kein Beitrag \\
\hline
\end{tabular}

Tabelle 2.3 Beiträge einzelner Ionen zu $K_{1}$ nach [13, 17].

\section{Induzierte Anisotropieenergie und Desakkommodation}

Bisher wurde bei der Beschreibung der magnetokristallinen Anisotropieenergie eine kubische Symmetrie vorausgesetzt (vgl. Gleichung 2.2). Beim Auftreten von Ionen- bzw. Leerstellendiffusion kommt es jedoch durch Wechselwirkung zwischen Kristallstruktur und magnetischer Struktur zu einer Verringerung der freien Energie und damit verbunden zur Stabilisierung der (spontanen) Magnetisierungsrichtung und zur Aufhebung der kubischen Symmetrie. Bei den Ferriten spielen hierbei insbesondere Kationen- und Kationen - Kationenleerstellen - Konfigurationen sowie Leerstellenpaare eine wichtige Rolle. Die für die Kationendiffusion notwendige Aktivierungsenergie liegt bei ca. $1 \mathrm{eV}$ bis $4 \mathrm{eV}$ [18-20] und ist damit ungefähr zehnmal so hoch wie bei der Elektronendiffusion. Die Stabilisierung der Bereichsstruktur durch Kationendiffusion ist mit den Phänomenen der induzierten Anisotropie und der Desakkommodation verbunden.

Die einachsige, bidirektionale induzierte Anisotropie lässt sich durch Magnetfeldtempern unterhalb der Curietemperatur erzeugen (induzieren) und überlagert sich der kubischen Anisotropie. Sie folgt der Beziehung $E_{U} / V=K_{U} \sin ^{2} \varphi$, wobei $\varphi$ den Winkel zwischen der induzierten Richtung und der Magnetisierungsrichtung angibt.

Findet der Stabilisierungsprozess ohne ein äußeres Magnetfeld statt, so spricht man von Desakkommodation. Die Anfangspermeabilität sinkt im Zeitraum $t_{2}-t_{1}$ mit zunehmender Stabilisierung der Domänen von $\mu_{i 1}$ auf $\mu_{i 2}$. Dies wird meist 
vereinfachend durch eine logarithmische Abhängigkeit der Form $\Delta \mu_{i}=-D_{F} \mu_{i 1}^{2} \cdot \log \left(t_{2} / t_{1}\right)$ mit dem Desakkommodationsbeiwert $D_{F}$ wiedergegeben.

Desakkommodation und induzierte Anisotropie lassen sich nicht eindeutig einer Ionenkonfiguration zuordnen. Vielmehr geht man von mehreren unterschiedlichen, sich überlagernden Mechanismen aus, an denen verschiedene Kationen bzw. Kationenleerstellen beteiligt sind, welche in Abhängigkeit von der magnetischen Vorzugsrichtung wirksam werden.

\subsubsection{Magnetoelastische Energie}

Einen weiteren Beitrag zur vom Magnetismus abhängigen freien Energie des Kristalls liefert die magnetoelastische Energie. Sie gibt die Verformung oder Magnetostriktion des Kristalls unter Wirkung eines Magnetfeldes wieder und verbindet die elastischen mit den magnetischen Eigenschaften. Die magnetoelastische Energie ist eng verwandt mit der magnetokristallinen Anisotropie und hat ihre Ursache in der Wechselwirkung zwischen den 3d-Orbitalen der Kationen und dem Kristallfeld des durch Spannungen verzerrten Kristallgitters.

Für den Fall der linearen Magnetostriktion, bei der keine Volumenänderung auftritt, führt man wie bei der Kristallanisotropieenergie die Richtungskosinusse $\alpha_{\mathrm{i}}$ zwischen Kristallachse $(i=1,2,3=x, y, z)$ und Magnetisierung ein. Für die in einem kubischen Kristall bei Sättigung auftretenden Längenänderungen ergibt sich der Zusammenhang:

$$
\begin{aligned}
\frac{\Delta l}{l}= & \frac{3}{2} \lambda_{100}\left(\alpha_{1}^{2} \beta_{1}^{2}+\alpha_{2}^{2} \beta_{2}^{2}+\alpha_{3}^{2} \beta_{3}^{2}-\frac{1}{3}\right)+ \\
& 3 \lambda_{111}\left(\alpha_{1} \alpha_{2} \beta_{1} \beta_{2}+\alpha_{2} \alpha_{3} \beta_{2} \beta_{3}+\alpha_{1} \alpha_{3} \beta_{1} \beta_{3}\right)+\ldots .
\end{aligned}
$$

Die Richtungskosinusse $\beta_{i}$ geben hierbei die Richtung relativ zu den Kristallachsen an, in welcher die Längenänderung erfolgt. Die Magnetostriktionskonstanten $\lambda_{100}$ und $\lambda_{111}$ geben die Längenänderung in [100]-Richtung bei Magnetisierung in [100]-Richtung bzw. die Längenänderung in [111]-Richtung bei Magnetisierung in [111]-Richtung wieder. Die Magnetostriktionskonstanten betragen bei MnZn- und NiZn-Ferriten ungefähr $10^{-5}$, können aber insbesondere bei kobalthaltigen Ferriten deutlich höher sein. Die Magnetostriktion besitzt wie die magnetokristalline Anisotropieenergie eine ausgeprägte Temperaturabhängigkeit und fällt zu höheren Temperaturen hin meistens ab. 
Für polykristalline Materialien berechnet man den Mittelwert der Magnetostriktionskonstanten bei Sättigung zu

$$
\lambda_{\text {Poly }}=\frac{2}{5} \lambda_{100}+\frac{3}{5} \lambda_{111}
$$

und die Längenänderung, die mit der Magnetisierungsrichtung den Richtungskosinus $\beta$ bildet, ergibt sich zu

$$
\Delta l / l=\frac{3}{2} \lambda_{\text {Poly }}\left(\beta^{2}-\frac{1}{3}\right)
$$

Die durch die Längenänderung erzeugte Spannungsenergie hängt von den $\alpha_{\mathrm{i}}$, dem Elastizitätsmodul $c_{\mathrm{ij}}$ des Kristalls und von der Dehnung $\varepsilon_{\mathrm{ij}}$ ab und lässt sich in ihrer Richtungsabhängigkeit genau wie die Magnetokristalline Anisotropie nach Gleichung 2.2 entwickeln.

Neben der durch die Magnetisierung hervorgerufenen Dehnung der Kristalle verursachen umgekehrt äußere Spannungen über ihre Wechselwirkung mit der Magnetostriktion einen zusätzlichen Beitrag zur Energie des Festkörpers. Für den Fall einer homogenen Zugoder Druckspannung $\sigma$, deren Richtung mit der Magnetisierung den Winkel $\vartheta$ bildet, berechnet sich die Magnetoelastische Energie zu

$$
\frac{E_{M E}}{V}=\frac{3}{2} \lambda_{P o l y} \sigma \sin ^{2} \vartheta
$$

Im realen Festkörper treten häufig Spannungen aufgrund von Gefügeinhomogenitäten, Fehlstellen, Versetzungen, Ausscheidungen, in Korngrenzen segregierten Phasen oder Materialgradienten auf. Sie wechselwirken über die Magnetoelastische Energie mit der magnetischen Struktur des Festkörpers und können die magnetischen Eigenschaften mitunter stark verschlechtern und insbesondere die Permeabilität der Materialien sowie die Güte $Q^{[\mathrm{i}]}$ verringern.

\subsubsection{Empirischer Ansatz zur Bestimmung der Anfangspermeabilität}

Von Smit und Wijn [12] wurde folgender empirischer Ansatz zur Berechnung der Anfangspermeabilität vorgeschlagen:

$$
\mu_{i}-1 \propto \frac{M_{S}^{2}}{E_{K}+E_{M E}+\ldots}
$$

[i] Die Güte $Q$ berechnet sich aus dem magnetischen Verlustwinkel $\delta_{\mu}$ gemäß $Q=1 / \tan \delta_{\mu}$ (vgl. die Abschnitte 2.3 und 3.4.1). 
Der Nenner beschreibt ganz allgemein den Einfluss einer effektiven Anisotropieenergie und kann gegebenenfalls durch weitere Terme der freien Energie des Ferrits ergänzt werden, wie z.B. der Formanisotropieenergie oder der induzierten Anisotropieenergie.

Mit Hilfe dieses Ansatzes lässt sich die Ausbildung der Permeabilitätsmaxima im Temperaturverlauf der Anfangspermeabilität erklären (Abbildung 2.4).

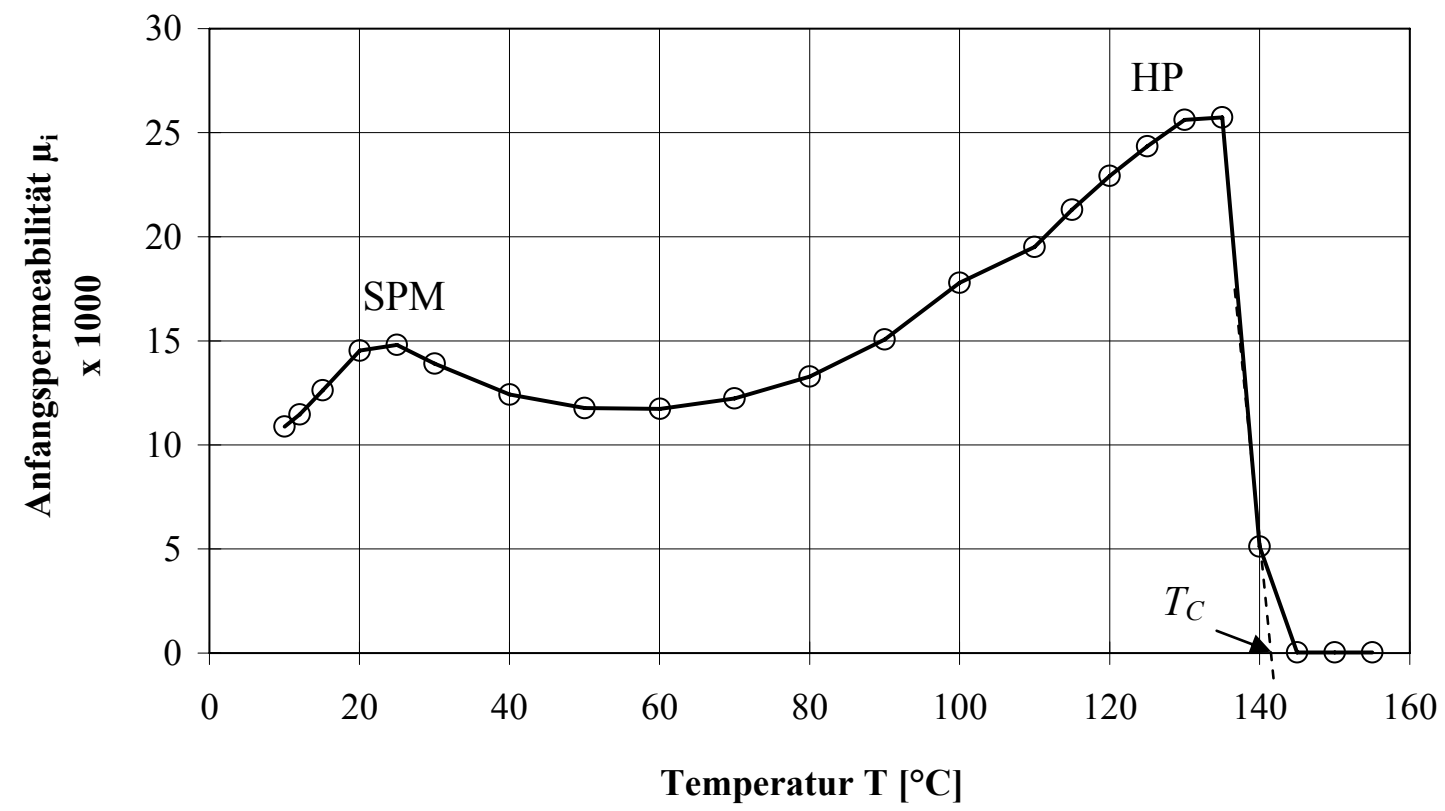

Abbildung 2.4 Temperaturverlauf der Anfangspermeabilität, gemessen an einem hochpermeablen MnZn-Ferrit mit sekundärem Permeabilitätsmaximum (SPM), Hopkinsonpunkt (HP) und Curietemperatur $\mathbf{T}_{\mathbf{C}}$.

Das sekundäre Permeabilitätsmaximum (SPM) und der Hopkinsonpunkt (HP) kommen durch ein Minimum der Kristallanisotropie zustande. Im ersten Fall kompensieren sich die Anteile der einzelnen Ionen aufgrund ihrer unterschiedlichen Temperaturabhängigkeit, im zweiten Fall kommt es aufgrund der Auflösung der magnetischen Ordnung vor Erreichen der Curietemperatur $T_{C}$ zu einem Minimum der magnetischen Anisotropie.

Allerdings hat dieser einfache Ansatz auch deutliche Grenzen, denn Gleichung (2.9) berücksichtigt nicht den in Abschnitt 2.1.7 beschriebenen Einfluss des Gefüges.

\subsubsection{Magnetische Domänen und Magnetisierungsprozess}

Wie von den ferromagnetischen Materialien bekannt, bilden sich auch bei den Weichferriten magnetische Domänen oder Weißsche Bezirke aus [21, 22]. Sie reduzieren das Streufeld und tragen damit zu einer Verringerung der gesamten freien Energie bei. Art und Größe der Domänen, sowie Richtung und Breite der zwischen den Domänen 
befindlichen Blochwände werden von den oben erwähnten magnetischen Eigenschaften und der Mikrostruktur bestimmt [11]. In einem anisotropen Kristall kubischer Symmetrie bilden sich wie in Abbildung 2.5 a) gezeigt $180^{\circ}$ - und $90^{\circ}$-Blochwände aus. Innerhalb der Domänen sättigt das Material in der leichten Magnetisierungsrichtung, ohne dass ein Streufeld in den Außenraum tritt.

a)

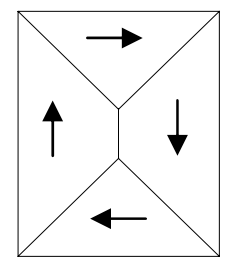

Abbildung 2.5 b)

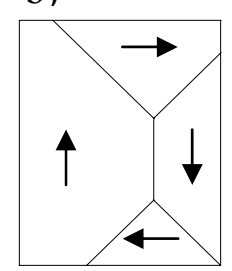

c)

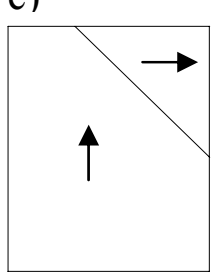

d)

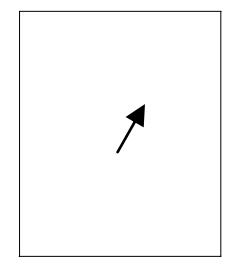

Richtung des äußeren Feldes $H_{a}$

Modellvorstellung des Magnetisierungsprozess für einen anisotropen Einkristall kubischer Symmetrie. Die Magnetisierung nimmt von a) nach d) zu.

Die Energie $\gamma_{B W}$ und die Breite der Blochwand $d$ können nach [12] durch die effektive Austauschkonstante $A^{\prime}$ (in $\mathrm{J} / \mathrm{m}$ ), die normierte Sättigungsmagnetisierungen $M_{S} / M_{S 0}$ $\left(M_{S 0}=\left.M_{S}\right|_{T=0}\right)$ und die Anisotropiekonstante $K_{1}$ abgeschätzt werden:

$$
\gamma_{B W}=p \frac{M_{S}}{M_{S 0}} \sqrt{A^{\prime} K_{1}}
$$

wobei $p \approx 1$ für $90^{\circ}$ Wände und $p \approx 2$ für $180^{\circ}$ Wände gilt.

$$
d=q \frac{M_{S}}{M_{S 0}} \sqrt{\frac{A^{\prime}}{K_{1}}},
$$

mit $q \approx 3 \ldots 20$ (von der kristallografischen Lage der Blochwand abhängiger Faktor).

Mit abnehmender Anisotropie $\left(K_{1} \rightarrow 0\right)$ nimmt auch die Blochwandenergie ab und die Breite $\mathrm{zu}$, bis sich im magnetisch isotropen Kristall die Magnetisierung kontinuierlich über das gesamte Volumen ändert. Mittels eines magnetischen Kraftmikroskops durchgeführte Untersuchungen [22] belegen Blochwandbreiten von ca. 0,2-1,5 $\mu \mathrm{m}$, wobei der erste Wert an einem niederpermeablen NiZn-Ferrit mit Kobaltdotierung $\left(\mathrm{Ni}_{0,49} \mathrm{Zn}_{0,49} \mathrm{Co}_{0,02} \mathrm{Fe}_{1,90} \mathrm{O}_{3,85}, \mu_{i}=100\right)$ und der zweite Wert an einem MnZn-Ferrit der Zusammensetzung $\mathrm{Mn}_{0,60} \mathrm{Zn}_{0,35} \mathrm{Fe}_{2,05} \mathrm{O}_{4}$ gemessen wurde. Desweiteren bestätigen die Untersuchungen die Existenz der in Abbildung 2.6 a) gezeigten, an Korngrenzen abknickenden Blochwände. Allerding erfolgt der Einbau solcher intrakristalliner Blochwände nur bis zu einer kritischen Korngröße von ca. 3 - $4 \mu \mathrm{m}$. Nach deren Unterschreiten treten wahrscheinlich nur noch Eindomänen-Körner auf [21, 23-25].

Weitere experimentell vor allem an Metalloberflächen belegte Modellvorstellungen [11] betreffen die Wechselwirkung der magnetischen Domänen mit intrakristallinen Poren und 
sind in Abbildung 2.6 b) gezeigt. Es handelt sich zum einen um die schon von Néel [26] postulierten dolchförmigen Domänen, welche das Entstehen von Streufeldern verhindern. Zum anderen finden Blochwände an den Poren eine energetische Minimumslage, man spricht in diesem Zusammenhang auch vom „Blochwand-Pinning“. In einem entmagnetisierten Kristall nehmen die Blochwände stets solche energetischen Minimumslagen ein, die sich durch die in Abschnitt 2.1.2 beschriebenen Diffusionsvorgänge weiter vertiefen. Zudem können innere Spannungszustände über eine Änderung der magnetoelastischen Energie die Domänen- und Blochwandstruktur beeinflussen.

a)

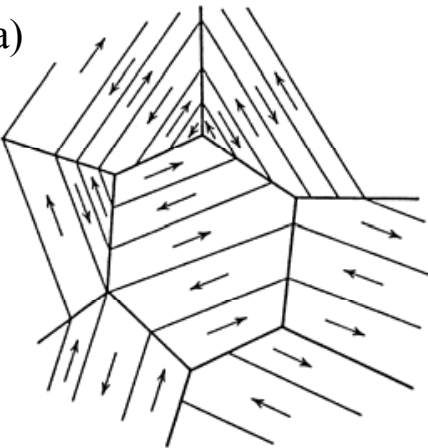

b)

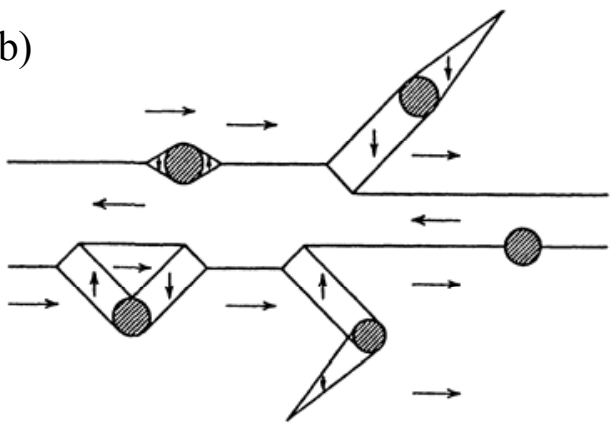

Abbildung 2.6 Modellvorstellungen zur Wechselwirkung von magnetischer Struktur und Mikrostruktur. a) Domänen im polykristallinen Gefüge, b) Wechselwirkung von Blochwänden mit unmagnetischen Ausscheidungen oder Poren. Blochwände werden festgehalten (Blochwand-Pinning) und es bilden sich dolchförmige Domänen [11].

Nach einer u.a. auf A. Globus [27] zurückgehenden Modellvorstellung werden unter der Wirkung eines schwachen äußeren Feldes $H_{a}$ die Domänenwände zuerst nur ausgewölbt, bleiben aber an ihren Verankerungspunkten fixiert und gehen bei abnehmendem Feld wieder in ihre Ausgangslagen zurück. Die Anfangspermeabilität $\mu_{i}$ wird als Grenzwert für reversible Ummagnetisierungsprozesse und verschwindende Felder definiert:

$$
\mu_{i}=\lim _{\hat{H} \rightarrow 0} \frac{\hat{B}}{\hat{H}}=\left.\frac{d B}{d H}\right|_{H, B \approx 0} .
$$

Im Allgemeinen kommt es mit Anwachsen der Feldstärke im zunehmenden Maße zum Losreißen und Verschieben der Domänenwände durch sprunghafte, irreversible Umklappprozesse. Reversible und irreversible Prozesse treten meist gemeinsam auf, wobei die irreversiblen Prozesse mit zunehmendem $H$-Feld zu- und die reversiblen Prozesse abnehmen. Ausnahme bilden die Ferrite mit Perminvar-Effekt ${ }^{[\mathrm{i}]}$, bei denen bis zu einer kritischen Feldstärke nur reversible Prozesse stattfinden und sich die Permeabilität

[i] Der Ausdruck Perminvar besagt, dass die Permeabilität invariant gegenüber Feldänderungen unterhalb der kritischen Feldstärke ist. 
praktisch nicht ändert.

Für höhere Aussteuerungen definiert man die Amplitudenpermeabilität $\mu_{a}$ analog zur Anfangspermeabilität $\mu_{i}$ als (vgl. Abbildung 2.7):

$$
\mu_{a}=\frac{\hat{B}}{\hat{H}}
$$

Aus dem Verlauf der magnetischen Neukurve in Abbildung 2.7 lässt sich die Änderung der Amplitudenpermeabilität mit dem äußeren Feld $H$ konstruieren. Sie steigt mit wachsender Aussteuerung an, erreicht ein Maximum und fällt dann wieder ab.

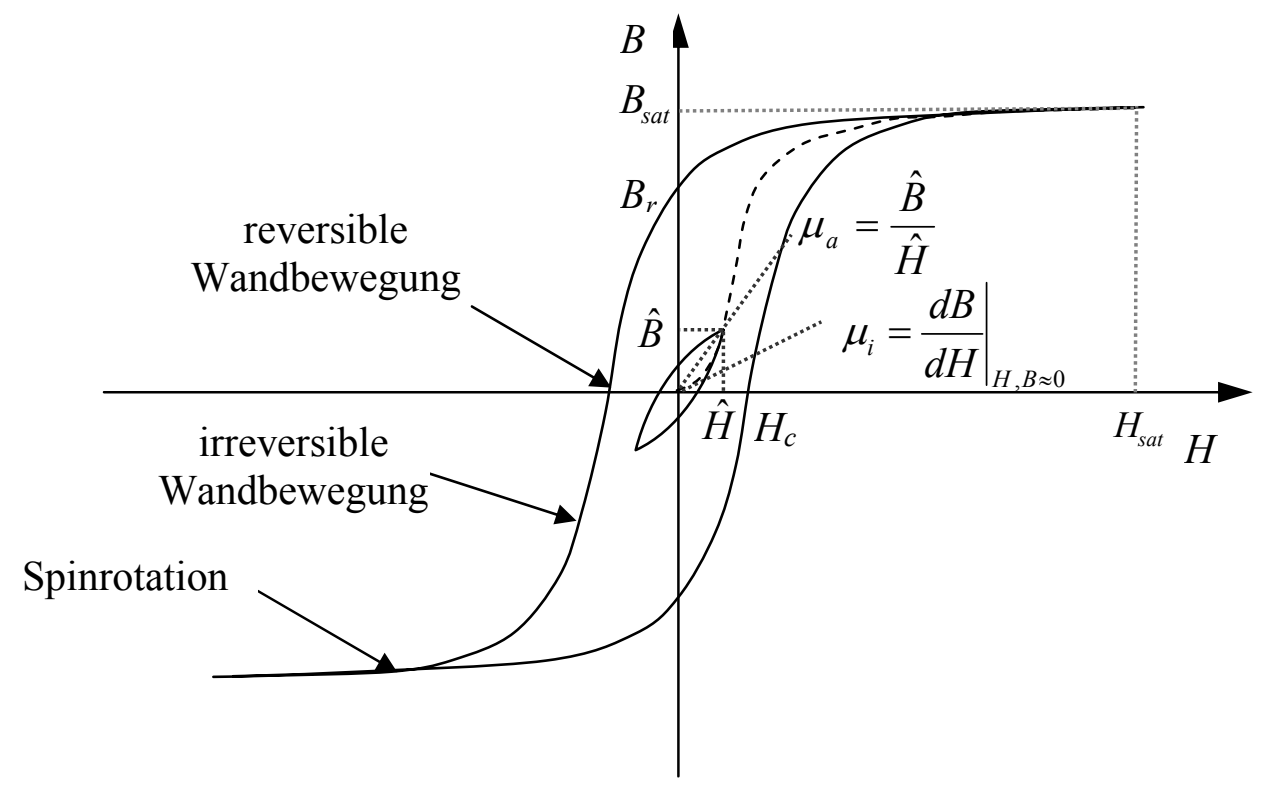

Abbildung 2.7 Hysteresekurve eines weichmagnetischen Werkstoffes mit Sättigungsmagnetisierung $B_{\text {sat }}$, Koerzitivfeld $H_{c}$, Remanenz $B_{r}$, sowie der Anfangs- und Amplitudenpermeabilität $\mu_{i}$ bzw. $\mu_{a}$.

Neben der Blochwandbewegung kann sich die Magnetisierung auch durch Spinrotation erhöhen. Die magnetischen Momente werden unter der Kraft des äußeren Feldes aus ihrer Vorzugsrichtung gedreht.

Abbildung 2.5 zeigt idealisiert die Verschiebung der Blochwände bei der Magnetisierung eines porenfreien Kristalls kubischer Symmetrie mit starker Anisotropie. Hier setzt Spinrotation erst dann ein, wenn sich eine Zunahme der Magnetisierung in Richtung des äußeren Feldes nicht mehr durch Blochwandbewegung erreichen lässt (Abbildung 2.5, Teilabbildung c).

Im Allgemeinen sind Spinrotation und Blochwandbewegung jedoch konkurrierende Prozesse, die in Abhängigkeit von der magnetokristallinen Anisotropieenergie und der Mikrostruktur zumindest teilweise auch gemeinsam auftreten.

Erfolgt die gesamte Magnetisierung $M$ in Richtung des äußeren Feldes, so ist die Sättigungsinduktion $B_{\text {sat }}$ erreicht (vgl. Abbildung 2.7). 


\subsubsection{Ummagnetisierungsverluste}

Beinahe immer werden weichmagnetische Ferrite mit magnetischen Wechselfeldern zyklisch ausgesteuert. Hierbei treten Ummagnetisierungsverluste auf. Zum einen sind dies die Hystereseverluste, welche durch die für einen Ummagnetisierungszyklus benötigte Arbeit gegeben sind:

$$
P_{h}=f \oint H d B
$$

$\left(P_{h}=\right.$ Hystereseverlustleistung $\left[\mathrm{W} / \mathrm{m}^{3}\right], f=$ Frequenz $)$.

Des Weiteren treten aufgrund des endlichen elektrischen Widerstandes des Magnetmaterials induzierte Wirbelströme auf, die $\mathrm{zu}$ Wirbelstromverlusten führen. Wirbelstromverluste nehmen quadratisch mit der Frequenz zu. Sie berechnen sich für intrakristalline Wirbelströme zu

$$
P_{w} \propto f^{2} \hat{B}^{2} \frac{\bar{X}^{2}}{\rho}
$$

$\left(P_{w}=\right.$ Wirbelstromverlustleistung $\left[\mathrm{W} / \mathrm{m}^{3}\right], \rho=$ spez. Widerstand im Korninneren, $\hat{B}=$ magnetische Induktion, $f=$ Frequenz, $\bar{X}=$ mittlere Korngröße).

Hysterese- und Wirbelstromverluste spielen bei der Ummagnetisierung von Ferriten im Frequenzbereich bis $1 \mathrm{MHz}$ die entscheidende Rolle. Zur Separation der beiden Verlustanteile wird folgender einfacher Ansatz mit dem Hystereseverlustfaktor $\alpha_{h}$ und dem Wirbelstromverlustfaktor $\alpha_{w}$ gewählt:

$$
P=\alpha_{h} f+\alpha_{w} f^{2}
$$

$\left(\alpha_{h}\right.$ in $\left[\mathrm{Ws} / \mathrm{m}^{3}\right], \quad \alpha_{w}$ in $\left.\left[\mathrm{Ws}^{2} / \mathrm{m}^{3}\right]\right)$. Weitere Verlustmechanismen wie z.B. die dielektrischen Verluste oder Verluste aufgrund der magnetischen Resonanz spielen bei den betrachteten Frequenzen $<1 \mathrm{MHz}$ keine Rolle. Sie kommen aber bei Mikrowellenfrequenzen zum Tragen (vgl. Abschnitt 2.3).

\subsubsection{Mikrostruktur und magnetische Eigenschaften}

Die in den vorigen Abschnitten beschriebenen magnetischen Eigenschaften werden wesentlich durch das Gefüge bestimmt. Entsprechend kommt es für eine bestimmte Applikation nicht nur darauf an, die Zusammensetzung der Spinellphase auf eine bestimmte Eigenschaft, z.B. eine hohe Sättigungsmagnetisierung, eine hohe Anfangspermeabilität oder einen hohen spezifischen Widerstand, zu optimieren, sondern 
es ist in gleicher Weise wichtig, die Mikrostruktur, Poren, Körner, sowie die Verteilung etwaiger Fremdphasen optimal an diese Anwendungen anzupassen.

Im Allgemeinen lassen sich folgende Regeln für eine optimale Mikrostruktur angeben:

1. Die Mikrostruktur sollte möglichst dicht und frei von größeren Ausscheidungen an Fremdphasen bzw. agglomerierten Poren sein.

2. Ideal ist eine gleichmäßige Korngrößenverteilung. Stark bimodale Verteilungen bzw. das Auftreten diskontinuierlich gewachsener Körner (vgl. 2.2.1) verschlechtern die magnetischen Eigenschaften.

3. Zum Erreichen hoher Permeabilitäten und Sättigungen muss die Porosität möglichst gering sein. Poren reduzieren die Beweglichkeit der Blochwände (Pinning-Effekt) und als unmagnetisches Volumen die Sättigung des Materials.

4. Korngrenzen haben häufig einen ähnlichen Effekt. Die Permeabilität reduziert sich aufgrund einer verringerten Beweglichkeit intrakristalliner Blochwände (Modell von Globus [27]) und wegen der Zunahme der inneren Scherung (NMGB-Modell ${ }^{[\mathrm{i}]}$ von Johnson und Visser [28]). Beide Effekte sind experimentell belegt. Der erste führt zu einer linearen Zunahme der Permeabilität mit der Korngröße und wurde z.B. durch Untersuchungen von Röss und Hanke [29] bestätigt, die bei einem weitestgehend porenfreien grobkristallinen Gefüge mit Korngrößen bis zu $100 \mu \mathrm{m}$ ein lineares Anwachsen der Permeabilität mit der Korngröße auf $\mu_{i}=40000$ nachweisen.

Der zweite kommt insbesondere bei kleinen Korngrößen in der Nähe der für eindomänige Körner typischen Durchmesser von 3 - $4 \mu \mathrm{m}$ zum Tragen [21, 23] und führt dort zu einem verhältnismäßig starken Permeabilitätsabfall.

5. Andererseits erhöhen sich bei zu hohen Korngrößen $\bar{X}$ die intrakristallinen Wirbelstromverluste. Diese spielen bei Frequenzen $>300 \mathrm{kHz}$ eine wichtige Rolle. Bei Materialien, welche zur Leistungsübertragung bei diesen Frequenzen eingesetzt werden, ist deshalb ein möglichst feinkörniges Gefüge mit Korngrößen $<10 \mu \mathrm{m}$ vorteilhaft.

6. Korngrenzen bilden eine natürliche Barriere für den Strom. Koops [30] beschreibt das Verhalten eines polykristallinen Gefüges als elektrische Schaltung, bestehend aus Korngrenzenkapazitäten und Widerständen.

Ein möglichst hoher Korngrenzenwiderstand lässt sich durch die Segregation von Fremdphasen in der Korngrenze erreichen und reduziert die intergranularen Wirbelströme. Als Dotierungen werden zu diesem Zweck häufig Kalzium- und 
Siliziumoxid eingesetzt, die als Glasbildner amorphe Kornzwischenphasen erzeugen oder sich in Tripelpunkten anreichern [31]. Die Zusammensetzung, Struktur und Verteilung der Fremdphasen spielt eine wichtige Rolle und wird auch durch den keramischen Prozess, insbesondere durch die Pulveraufbereitung und das Sinterregime beeinflusst [32 - 34].

7. Defekte und insbesondere Poren erhöhen durch Blochwand-Pinning sowohl die Koerzitivfeldstärke als auch die Remanenz des Ferrits und damit die Hystereseverluste.

\subsection{Gefügebildungs- und Kornwachstumsprozesse}

Die meisten aktuellen Untersuchungen zum Gefügebildungsprozess beschäftigen sich mit technologischen Fragestellungen wie beispielsweise dem Einfluss feinteiliger Ausgangsprodukte oder der für die Multilayerfertigung wichtigen Absenkung der Sintertemperatur [35].

Die Probleme eines exakten physikalischen Zugangs liegen bei den MnZn-Ferriten insbesondere in der nach der Kalzination (vgl. Abschnitt 3.2) vorhandenen Mehrphasigkeit des Systems und in den während des Aufheizvorganges stattfindenden komplexen Phasenbildungs- und Phasenumwandlungsprozessen begründet. Des Weiteren kann das Dotierungssystem das Sinterverhalten mitunter in beträchtlichem Maße beeinflussen [36].

Auch wenn die Unterschiede zu den in der Literatur untersuchten einphasigen Systemen und erst recht $\mathrm{zu}$ den auf Kugeln und Kugelschüttungen beruhenden Modellsystemen groß sind, so lassen sich doch Einzelheiten auf das Sintern eines mehrphasigen Grünkörpers ${ }^{[i]}$ mit seiner inhomogenen Dichteverteilung und seinen stark defektbehafteten Kontaktzonen übertragen. An dieser Stelle soll nur auf die wichtigsten Aspekte der Sintertheorie eingegangen werden, ausführliche weiterführende Beschreibungen finden $\operatorname{sich}$ in $[37-41]$.

\subsubsection{Stadien des Sinterprozesses}

Man unterscheidet gemeinhin drei unterschiedliche Stadien [37,38]. Das Anfangsstadium, welches durch ein offenporiges Gefüge und die Bildung von

[i] Als Grünkörper oder „Grünling“ werden keramische Formkörper vor dem Sintern bezeichnet. Sie bestehen aus der kompaktierten keramischen Masse und organischen Bestandteilen (z.B. Binde- und Gleitmittel). 
Sinterhälsen gekennzeichnet ist, das Zwischenstadium, während dem die einzelnen Pulverteilchen bis auf Porenkanäle versintern und das Endstadium, in welchem eine geschlossene Porosität vorherrscht.

\section{$\underline{\text { Das Anfangsstadium }}$}

Dieses ist geprägt von einer noch weitestgehend schwindungsfreien Verstärkung der Kontakte zwischen Presspulverteilchen, einer Glättung der vorhandenen Oberflächenrauigkeit bzw. von einer Verrundung von Oberflächen.

Im Zweikugelmodell kommt es zur Ausbildung von Sinterhälsen zwischen den kugelförmigen Festkörpern. Ursache hierfür sind unterschiedliche und von der Gleichgewichtskonzentration $C_{V 0}$ abweichende Leerstellenkonzentrationen, die $\mathrm{zu}$ einer Materialdiffusion hin zur Kontaktstelle führen. In Verallgemeinerung der KelvinThomson-Gleichung ist die Leerstellenkonzentration $C_{V}$ in der Nähe einer gekrümmten Oberfläche mit Radius $R$ und Oberflächenenergie $\gamma_{P}$ nach [38] gegeben durch:

$$
C_{V}=C_{V 0}\left(1+\frac{2 \gamma_{P} \Omega}{R k_{B} T}\right)
$$

( $\Omega=$ Leerstellenvolumen, $k_{B}=$ Boltzmannkonstante, $T=$ Temperatur).

Dies bedeutet für die in Abbildung 2.8 dargestellte Situation eines Zweiteilchenmodells, dass im konkaven Bereich des Sinterhalses, mit aus Sicht des umgebenden Gasraumes positivem Radius $r$, die Leerstellenkonzentration größer, im konvexen Bereich mit Radius $-X / 2$ hingegen kleiner als die Gleichgewichtskonzentration ist. Es kommt folglich zu Leerstellenströmen aus dem oberflächennahen Bereich der Kontaktzone bzw. zur Diffusion von Ionen oder Atomen in die umgekehrte Richtung.

Der Materialtransport erfolgt durch Oberflächendiffusion, Volumenselbstdiffusion und im Falle polykristalliner Kugeln auch durch Korngrenzendiffusion. Wie Schatt [38] bemerkt, führen zwar alle in Abbildung 2.8 gezeigten Diffusionswege zum Anwachsen des Sinterhalsdurchmessers, es kommt aber nur bei Diffusion in der Kontaktfläche bzw. im angrenzenden Volumen (Diffusionsweg b) zu einer Annäherung der Kugelzentren.

Die Ausbildung von Kontaktstellen ist also nicht unbedingt mit einer starken Schwindung verbunden. In Übereinstimmung hiermit findet man bei MnZn-Ferriten nach einer einstündigen Temperaturbehandlung bei $600^{\circ} \mathrm{C}$ zwar eine merkliche Verfestigung der Kerne, jedoch nur eine Schwindung von ca. $2 \%$. 

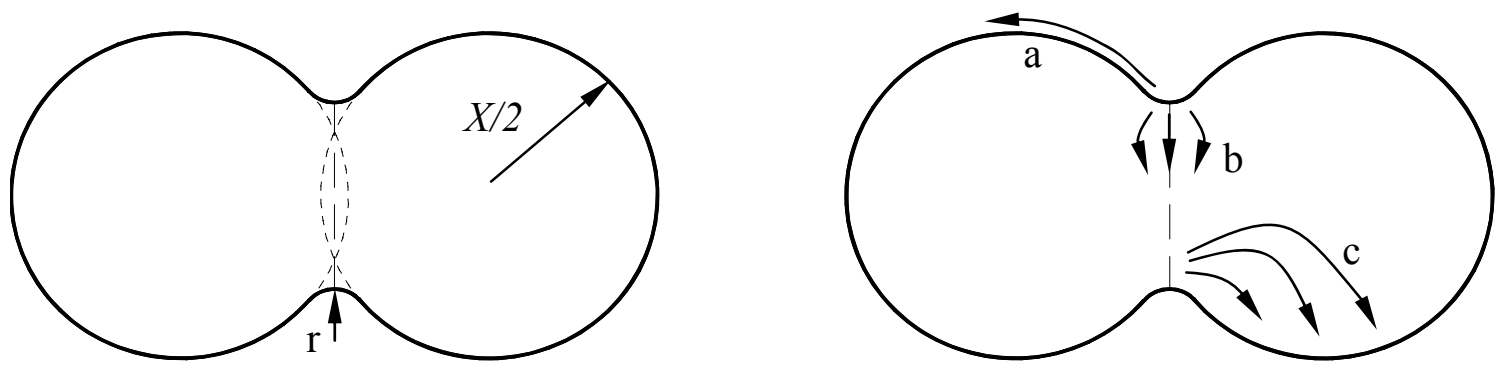

Abbildung 2.8 Zweiteilchenmodell für das Anfangsstadium. Unter der konkaven Oberfläche mit Radius $r$ bildet sich eine Leerstellenquelle, unter der konvexen Oberfläche mit Radius $X / 2$ eine Leerstellensenke. Über Oberflächendiffusion (a), Diffusion entlang der Kontaktstelle (b), Volumenselbstdiffusion bzw. Korngrenzendiffusion (c), entsteht ein Leerstellenfluss aus der Kontaktzone heraus [38, 41].

\section{Das Zwischen-oder Schwindungsintensivstadium}

Wie die Anfangsphase, so findet auch die Zwischenphase zumindest größtenteils während des Aufheizens statt. Die auftretenden Kapillarkräfte bewirken eine starke Verdichtung des Sinterkörpers, was sich auch in dem Namen „Schwindungsintensivstadium“ niederschlägt. Aus dem relativ großen, freien Volumen um die Sinterhälse entstehen Porenkanäle (Abbildung 2.9), welche die noch verbleibenden Poren miteinander verbinden. Die Sinterhälse selbst erweitern sich zu Kontaktkorngrenzen. Am Ende des Schwindungsintensivstadiums stehen der Übergang zum geschlossenporigen Gefüge und das beginnende Kornwachstum.

Die Kapillarspannung wirkt hierbei als eigentliche Triebkraft des Sinterprozesses und ist dabei ganz analog zu einem von außen wirkenden hydrostatischen Druck aufzufassen [37].

Nach [38] ist die mittlere Kapillarspannung $\bar{p}$ für eine Kugelschüttung mit mittlerem Kugeldurchmesser $\bar{X}$ gegeben durch

$$
\bar{p}=A \frac{2 \gamma-\gamma_{B}}{\bar{X}} \Theta
$$

( $A$ = dimensionslose Konstante, $\gamma=$ Oberflächenenergie Korn-Gasphase, $\gamma_{B}=$ Korngrenzenenergie, $\Theta=V_{\text {pore }} / V_{\text {gesamt }}=$ Volumenanteil der Porosität). 


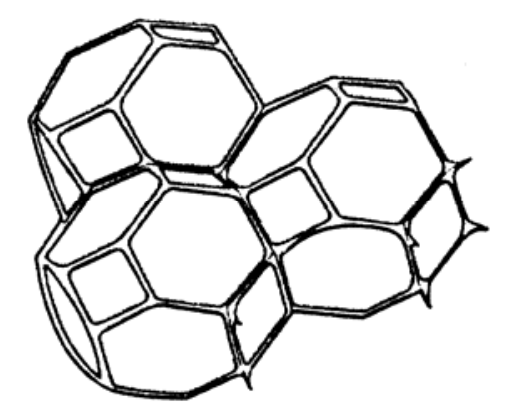

Abbildung 2.9 Gefügemodell für das Zwischenstadium (Tetrakaidekaedermodell) mit den in den Ecken befindlichen Poren und verbindenden Porenkanälen (Coble-Modell nach $[39,42])$.

Als Mechanismen für die Schwindung werden erwähnt:

1. Das Diffusionskriechen oder diffusionsviskose Fließen, bei dem die Schwindung durch eine Atomdiffusion entlang der Korngrenzen (Coble-Mechanismus) oder durch das den Korngrenzen benachbarte Volumen (Nabarro-Herring-Mechanismus) hervorgerufen wird [42]. Die Schwindungsgeschwindigkeit $\dot{\varepsilon}$ schätzt man nach Schatt [38] ab, indem die den Kriechmechanismus auslösende Spannung $\sigma$ durch den Kapillardruck $\bar{p}$ aus Gleichung 2.18 ersetzt wird:

$$
\begin{array}{ll}
\dot{\varepsilon} \approx B_{1} \bar{p} \frac{D_{V} \Omega}{k_{B} T} \frac{1}{\bar{X}^{2}} & \\
\dot{\varepsilon} \approx B_{2} \bar{p} \frac{b D_{B} \Omega}{k_{B} T} \frac{1}{\bar{X}^{3}} & \text { (Nabarro-Herring-Mechanismus) },
\end{array}
$$

$\left(D_{V}=\right.$ Volumenselbstdiffusionskoeffizient, $D_{B}=$ Korngrenzendiffusionkoeffizient, $b=$ wirksame Breite der Korngrenze, $\Omega=$ Leerstellenvolumen, $B_{1} \approx 10$ und $B_{2} \approx 150$ sind Geometriefaktoren).

Im Allgemeinen kann davon ausgegangen werden, dass beim Sintern beide Mechanismen zur Wirkung kommen, wobei der Nabarro-Herring-Mechanismus bei höheren Temperaturen bevorzugt wird.

2. Das Versetzungskriechen oder versetzungsviskose Fließen, bei dem die Viskosität durch kletternde Stufenversetzungen verursacht wird und bei dem die Schwindungsgeschwindigkeit für eine mittlere Versetzungsdichte $\bar{N}_{V}$ gegeben ist durch

$$
\dot{\varepsilon} \approx \bar{p} \frac{D_{V} \Omega}{k_{B} T} \bar{N}_{V}
$$

( $\bar{p}=$ Kapillardruck, $\quad D_{V}=$ Diffusionskonstante der Volumenselbstdiffusion, $\Omega=$ Atomvolumen). 
3. Die superplastische Verformung, bei der sich unter Spannung ganze Körner gegeneinander verschieben [43]. Superplastische Verformung führt zu einer guten Dehnabarkeit unter Zugbelastung, kann aber auch zu einer schnellen Verdichtung und zum Ausfüllen von Hohlräumen beitragen [38].

Die Defektstruktur der Korngrenze, Versetzungen und das Entstehen und Ausheilen interkristalliner Poren sowie die Korngrenzendiffusion spielen eine wichtige Rolle. Bei Keramiken ist das Auftreten superplastischer Verformung typischerweise an Primärkorngrößen $<0,5 \mu \mathrm{m}$ gebunden [44]. Bei den hier untersuchten Ferriten treten nur mittlere Primärkorngrößen von ca. $1 \mu \mathrm{m}$ auf, weshalb echte Superplastizität nur lokal, aufgrund des vorhandenen Feinkornanteils vorkommt. Trotzdem sind die bei der superplastischen Verformung auftretenden Prozesse direkt auf die im Schwindungsintensivstadium stattfindenden Teilchenbewegungen übertragbar.

Schatt [38] belegt, dass die Diffusionskriechvorgänge nicht ausreichen, um die an Metallen bei hohen Aufheizraten gemessenen Schwindungsraten zu erklären. Vielmehr kommt es aufgrund hoher Versetzungskonzentrationen zur Ausbildung niedrigviskoser Kontaktbereiche zwischen den Teilchen und $\mathrm{zu}$ einer schnellen Verdichtung durch Abgleiten ganzer Teilchen sowie durch Materialumfällung in der Kontaktzone (vgl. Abbildung 2.10). Wie Positronenannihilationsmessungen zeigen, sind beide Prozesse mit einer Abnahme der Versetzungsdichte verbunden.

Die Schwindungsgeschwindigkeit $\dot{\varepsilon}$ hängt nicht nur von der Sintertemperatur $T$, sondern wesentlich von der Aufheizgeschwindigkeit $d T / d t$ ab. Ursache hierfür ist das Ausheilen von Defekten, insbesondere von Versetzungen, welches bei langsamem Aufheizen zu einer Verringerung der Defektdichte führt und dadurch das Abgleiten der Körner und die weitere Verdichtung erschwert. Das Phänomen einer schnellen Verdichtung bei großen Aufheizgeschwindigkeiten wird als schnelle, nichtisotherme Schwindung bezeichnet und ist sowohl für Metalle [45] als auch für Keramiken beschrieben [46-48]. 


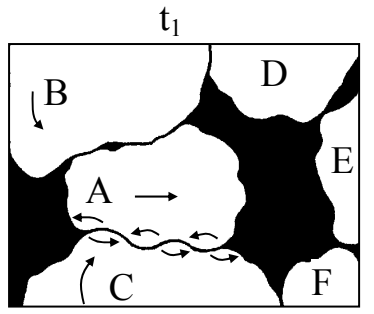

$\mathrm{t}_{2}$

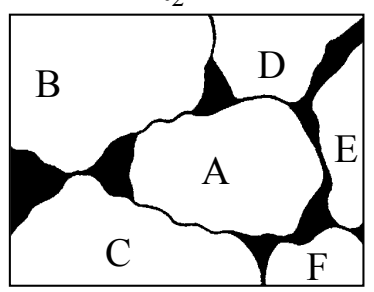

a)

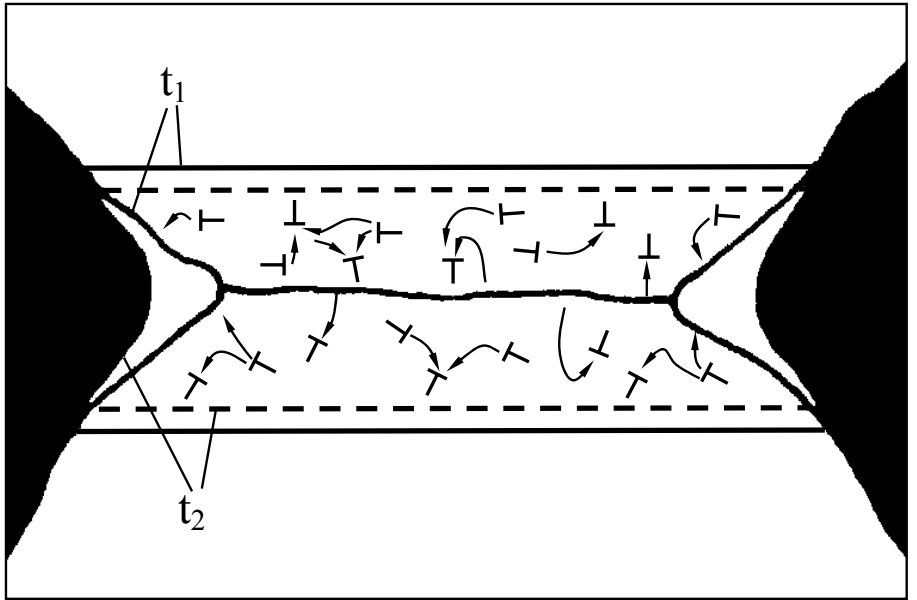

b)

Abbildung 2.10 Modellvorstellungen zur Verdichtung im Schwindungsintensivstadium durch versetzungsviskoses Fließen. Sowohl bei der Teilchenbewegung a), als auch bei der Teilchenannäherung durch Materialumfällung in der Kontaktzone b), ist eine niedrige Viskosität der Kontaktbereiche von Bedeutung (nach [38]).

\section{$\underline{\text { Das Spätstadium }}$}

Mit dem Verschwinden der offenen Porosität beginnt das Spätstadium des Sinterns. Die Dichte liegt in diesem Stadium idealerweise bei ca. $90 \%$ der theoretischen Dichte. Der Kapillardruck ist für ein nun geschlossenes System kugelförmiger Poren mit mittlerem Radius $\bar{R}$ und der Oberflächenenergie im Poreninneren $\gamma_{P}$ gegeben durch:

$$
\bar{p}=A \frac{2 \gamma_{P}}{\bar{R}} \Theta
$$

$\left(\mathrm{A}=\right.$ dimensionslose Konstante). Die Poren können durch Diffusion abgebaut werden ${ }^{[\mathrm{i}]}$. Wichtig ist hierbei die Unterscheidung zwischen inter- und intrakristalliner Porosität. Der Abbau interkristalliner Poren ist wegen des günstigeren Diffusionsweges entlang der Korngrenze meistens beschleunigt. Hingegen bauen sich intrakristalline Poren aufgrund der langsameren Volumendiffusion im Korninneren insbesondere bei großen Abständen zu den Korngrenzen nur sehr langsam oder gar nicht ab. Es besteht sogar die Möglichkeit einer Zunahme einzelner intrakistalliner Poren durch Ostwaldreifung.

[i] In Abhängigkeit von der Sinteratmosphäre und den auftretenden Partialdrücken kann sich in den Poren sowohl Vakuum als auch Gas oder ein Gemisch unterschiedlicher Gase befinden.

Ferrite werden häufig unter Luftatmosphäre bei Normaldruck erwärmt, so dass beim Entstehen einer geschlossenen Porosität vermutlich ein Gasgemisch vorliegt, welches in seiner Zusammensetzung ungefähr der Luftatmosphäre entspricht. Allerdings ist die Diffusion der einzelnen Bestandteile unterschiedlich. Insbesondere wird sich aufgrund einer guten Sauerstoffdiffusion der Sauerstoffpartialdruck im Poreninneren im weiteren Verlauf des Sinterns schneller abbauen. 
In verstärktem Maße tritt nun auch Kornwachstum auf, dessen Kinetik sich nach [49] durch folgende verallgemeinerte phänomenologische Gleichung beschreiben lässt:

$$
\bar{X}^{n}-\bar{X}_{0}^{n}=\tilde{k}\left(t-t_{0}\right) \text { mit } \widetilde{k}=\widetilde{K} \exp \left(\frac{-Q}{k_{B} T}\right)
$$

( $\bar{X}=$ Korngröße zum Zeitpunkt $t, n=$ Exponent, $\bar{X}_{0}=$ Korngröße zum Zeitpunkt $t_{0}, \tilde{k}$, $\widetilde{K}=$ Konstanten in $m^{n} / s, Q=$ Aktivierungsenergie in $J$ ).

Nimmt man an, dass die Geschwindigkeit der Korngrenze $v_{B}$ durch die Korngrenzenbeweglichkeit $M_{B}$ und dem Druck auf die Korngrenze $p=\gamma_{B} \kappa$ gemäß

$$
v_{B}=M_{B} p=M_{B} \gamma_{B} \kappa
$$

gegeben ist $\left(\kappa=\left(\rho_{1}+\rho_{2}\right) / 2 \rho_{1} \rho_{2}=\right.$ mittlere Oberflächenkrümmung der Korngrenze mit den Krümmungsradien $\rho_{1}$ und $\rho_{2}, \gamma_{B}=$ Energie der Korngrenze), so folgt für den Exponenten $n=2$ und $\tilde{k} \propto \gamma_{B} M_{B}$. Diese quadratische Abhängigkeit lässt sich bei einphasigen, ungestörten Gefügen auch experimentell nachweisen. Beim Vorhandensein von Poren oder Ausscheidungen findet man häufig $n \approx 3$ [50]. Dies gilt auch für die in Abschnitt 2.2.3 behandelte Anwesenheit schmelzfließender Phasen und wurde hierfür von Lay theoretisch abgeleitet [51].

Insbesondere beim Vorhandensein unbeweglicher Ausscheidungen werden in der Literatur jedoch auch Werte von bis zu $n \approx 6$ angegeben [52].

Die Aktivierungsenergie $Q$ im Exponenten der Arrheniusfunktion ist durch den das Kornwachstum begrenzenden Diffusionsprozess gegeben, welches im Normalfall die Volumenselbstdiffusion der Sauerstoffionen ist. Paulus [49] bestimmt für MnZn-Ferrite $\mathrm{Q}=3,6 \mathrm{eV}$.

Einschränkend ist zu sagen, dass Gleichung 2.23 nur für den Fall des normalen oder kontinuierlichen Kornwachstums gilt.

\subsubsection{Diskontinuierliches Kornwachstum}

Anders stellt sich die Situation beim Auftreten von diskontinuierlichem Kornwachstum dar. Einzelne Körner wachsen deutlich schneller als die umgebende Matrix und breiten sich bis zu einer Kristallitgröße von mehreren Millimetern aus. Intergranulare Poren, die sich bei normalem Kornwachstum mit der Korngrenze verschieben, werden von der sich schnell bewegenden Korngrenze umwachsen. Diskontinuierliches Kornwachstum geht deshalb meist mit hoher intragranularer Porosität einher. 
Treten sowohl normal als auch diskontinuierlich gewachsene Körner auf, so bezeichnet man dieses Gefüge als Duplexstruktur.

Voraussetzung für diskontinuierliches Kornwachstum ist eine hohe Korngrenzengeschwindigkeit $v_{B}$ und eine hohe Instabilität der Korngrenze. Es ist zu beachten, dass der Korngrenzendruck nicht nur durch die Krümmung der Korngrenze gegeben ist, sondern auch durch intrinsische Korneigenschaften wie z.B. durch unterschiedliche Versetzungsdichten oder Leerstellenkonzentrationen. Aber auch äußere Einflüsse wie ein den Materialtransport bzw. das Kornwachstum stimulierender Temperaturgradient oder ein Magnetfeld bei magnetisch stark anisotropen Materialien können den Druck auf eine Korngrenze erhöhen $[53,54]$. Die Korngrenzenbeweglichkeit $M_{B}$ ist weiterhin stark von der Kornorientierung $[55,56]$ abhängig und wird aufgrund von Pinning-Effekten durch in der Korngrenze vorhandene Fremdphasen oder Poren reduziert [57]. Auch im Gitter gut lösliche Komponenten führen im Allgemeinen zu einer Verringerung der Korngrenzenbeweglichkeit (Solute-Drag-Mechanismus) [58].

Ein von Heuer, Harmer und Brooks [59 - 61] stammender Ansatz zur Beschreibung von diskontinuierlichem Kornwachstum geht vereinfachend von kugelförmigen in der Korngrenze liegenden Poren mit Radius $R$ aus und berechnet die Kraft, die eine Korngrenze maximal auf eine Pore ausüben kann, ehe diese sich losreißt zu

$$
\hat{F}_{P}=\pi R \gamma_{B}
$$

Definiert man ähnlich wie in (2.24) die Porenmobilität als Proportionalitätsfaktor zwischen Porengeschwindigkeit $v_{P}$ und der auf die Pore wirkenden Kraft $F_{P}$, so ergibt sich die maximale Geschwindigkeit, mit der eine Pore in der Korngrenze mitbewegt werden kann, zu:

$$
\hat{v}_{P}=M_{P} \hat{F}_{P}
$$

Diffusionsprozesse auf der Oberfläche der Pore sind für die Porenbeweglichkeit ausschlaggebend [59]. Sie berechnet sich zu:

$$
M_{P}=\frac{\delta D_{S} \Omega}{\pi R^{4} k_{B} T}
$$

$\left(D_{S}=\right.$ Diffusionskonstante der Oberflächendiffusion im Poreninneren, $\delta \approx \Omega^{1 / 3}=$ Oberflächenschicht in der die Diffusion stattfindet, $\Omega=$ Atomvolumen). Die in der Korngrenze liegenden Poren mit mittlerer Dichte $\Theta_{B}$ (in $1 / \mathrm{m}^{2}$ ) üben eine Haltekraft auf 
die Korngrenze aus. Für den Fall, dass sich die Korngrenze gerade noch nicht aus ihrer Verankerung löst (maximale Haltekraft) gilt

$$
v_{B}=M_{B}\left(\gamma_{B} \kappa-\hat{F}_{P} \Theta_{B}\right) .
$$

Die Stabilitätsbedingung für das Losreißen der Korngrenze aus ihrer Verankerung lautet $v_{B}<\hat{v}_{P}$ und diskontinuierliches Korwachstum kann folglich vermieden werden wenn gilt:

$$
\Theta_{B}+\frac{M_{P}}{M_{B}}>\frac{\kappa}{\pi R}
$$

Mit Gleichung 2.29 lassen sich viele Effekte, die zur Entstehung von diskontinuierlichem Kornwachstum führen, zumindest qualitativ erklären. Richtiger ist es allerdings, wie Hsueh et al. [62] und Svoboda und Riedel [63] die tatsächliche, von der Kugelform abweichende und von der Porengeschwindigkeit abhängige Porenform, zu berücksichtigen (vgl. Abbildung 2.11.).

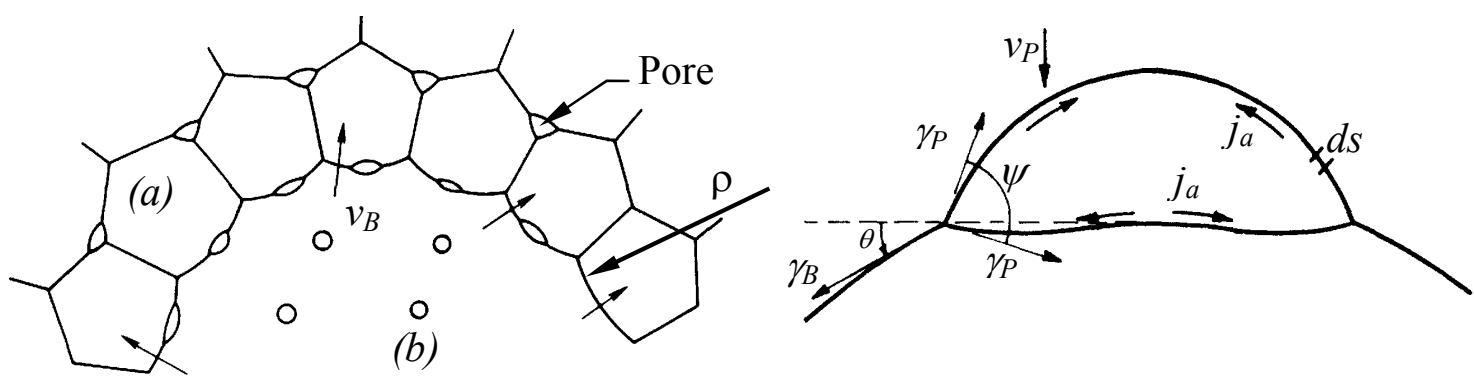

Abbildung 2.11 Modellvorstellung für die Entstehung von diskontinuierlichem Kornwachstum. Das normalgewachsene Gefüge (a) wird von einem diskontinuierlich wachsenden Korn (b) überwachsen. Die Korngrenzen mit Krümmungsradius $\rho$ werden in ihrer Bewegung durch interkristalline Poren gebremst. Die in der rechten Teilabbildung gezeigte Pore bewegt sich mit der Porengeschwindigkeit $v_{P}$ mit der Korngrenze nach unten. Die Porenbeweglichkeit wird durch die Diffusionsströme $j_{a}$ an der Porenoberfläche und durch die Porengröße bestimmt. (nach Hsueh, Evans und Coble) [62].

Der Öffnungswinkel der Pore $\psi$ ist durch die Korngrenzenenergie $\gamma_{B}$ und die Oberflächenenergie im Poreninneren $\gamma_{P}$ gegeben:

$$
\gamma_{B}=2 \gamma_{P} \cos \frac{1}{2} \psi
$$

Unabhängig vom Öffnungswinkel berechnen Svoboda und Riedel [63] für den kritischen (maximalen) Haltewinkel, bei dem die Pore gerade nicht mehr in der Korngrenze mitbewegt werden kann, einen Wert von $\theta_{k r i t},=36,5^{\circ}$. Die einfache Analyse von Heuer [60] findet hingegen für kugelförmige Poren einen kritischen Haltewinkel von $45^{\circ}$, während Hsueh et al. eine starke Abhängigkeit vom Öffnungswinkel $\psi$ und ein 
Anwachsen des kritischen Haltewinkels $\theta_{k r i t}$, von $13^{\circ}$ auf $23^{\circ}$ bei einer Verringerung des Öffnungswinkels $\psi$ von $100^{\circ}$ auf $60^{\circ}$ angeben.

\subsubsection{Flüssigphasensintern}

Unter Flüssigphasensintern versteht man im Allgemeinen die Beeinflussung des Verdichtungs- und Kornwachstumsprozesses durch die Anwesenheit einer flüssigen Phase während des Sintervorganges. Die flüssige Phase tritt meist während des Zwischen- oder Endstadiums durch Aufschmelzen einer Komponente auf und bleibt je nach System entweder nur temporär oder während des gesamten isothermen Sinterschrittes bis zum Unterschreiten des Schmelzpunktes beim Abkühlen existent. Die Menge an flüssiger Phase schwankt normalerweise von $<1 \%$ bis zu über $30 \%$. Bei den hier untersuchten Ferrit- $\mathrm{Bi}_{2} \mathrm{O}_{3}$ - und Ferrit- $\mathrm{MoO}_{3}$-Systemen liegt sie jedoch immer in einem sehr niedrigen Bereich von $<0,1$ Gew.\%.

Im Allgemeinen lassen sich folgende Mechanismen beim Flüssigphasensintern nachweisen $[38,64]$ :

1. Die Entstehung der Schmelze, wobei es sich sowohl um das Aufschmelzen einer Einzelkomponente als auch um ein eutektisches System handeln kann.

2. Die Benetzung der festen Bestandteile unter der Bedingung $\gamma_{S L}<\gamma_{S}+\gamma_{L}\left(\gamma_{S L}=\right.$ Kontakflächenenergie, $\gamma_{S}, \gamma_{L}=$ Oberflächenenergie der festen bzw. flüssigen Phase).

3. Das Eindringen der flüssigen Phase in die Kontaktstellen eines Pulverpresslings bzw. in die Korngrenzen eines polykristallinen Teilchens und die Desintegration (Vereinzelung) der Körner. Unter der Voraussetzung eines Überangebots an flüssiger Phase findet das Eindringen in eine Korngrenze oder Kontaktstelle der Energie $\gamma_{B}$ unter der Bedingung $2 \gamma_{S L}=\gamma_{B L}<\gamma_{B}$ statt $\left(\gamma_{B L}\right.$ ist die Korngrenzenenergie mit flüssiger Phase $)^{[\mathrm{i}]}$.

4. Die Lösung der festen in der flüssigen Phase, deren Wiederausscheidung und daraus resultierend eine beschleunigter Materialtransport in der Korngrenze und an der Oberfläche der Poren, was zu einer Zunahme der Korngrenzen- und Porenmobilität führt. (vgl. Gleichung 2.27). Für die das Kornwachstum beschreibende Gleichung 2.23 lässt sich ein Exponent $n=3$ bei Diffusion über die Schmelze als begrenzender Mechanismus ableiten.

[i] Es ist hierbei zu berücksichtigen, dass die Kontaktstellenenergie eines Pulverpressling aufgrund der beim Verdichtungsvorgang entstehenden hohen Defektdichten vergleichsweise hoch ist [38]. 
Parallel hierzu kann eine verbesserte Diffusion in der Korngrenze den Abbau interkristalliner Poren begünstigen.

5. Im Zwischenstadium kann die Anwesenheit einer flüssigen Phase über eine Beschleunigung der Korngrenzendiffusion das Coble-Kriechen beschleunigen (vgl. Gleichung 2.20). Aber auch das Abgleiten ganzer Teilchen, wie es bei der superplastischen Verformung und der schnellen nichtisothermen Verdichtung vorkommt, kann durch einen flüssigen Film auf der Teilchenoberfläche bzw. durch das Eindringen der flüssigen Phase in die Kontaktbereiche begünstigt werden.

6. Bei größeren Mengen an schmelzfließender Phase kommt es zu einem schnellen Ausfüllen der Poren durch die flüssige Phase und zu einer verhältnismäßig hohen Enddichte [65]. Bei geringeren Zusätzen kann jedoch auch ein gegenläufiger Effekt auftreten. So beschreiben Choi et al. eine Zunahme der intragranularen Porosität und eine Abnahme der Dichte bei $\mathrm{ZnO}$-Keramik mit einem Gehalt von 0,05 mol\% $\mathrm{Bi}_{2} \mathrm{O}_{3}$ [66].

Grundvoraussetzung für alle Mechanismen ist eine gute Benetzung der Festkörperoberfläche und das Eindringen der flüssigen Phase in die Korngrenzen bzw. Kontaktbereiche. Hieraus resultiert insbesondere aufgrund der Punkte 3. - 6. teilweise eine bessere Verdichtung. Es kann aber auch wegen der erhöhten Korngrenzenmobilität zu verstärktem Kornwachstum und zu einer Verringerung der Enddichte kommen. Zovas und German [58] nennen hierfür insbesondere drei Gründe:

1. Der Anteil an Korngrenzen sinkt mit Anwachsen der Korndurchmesser und somit reduziert sich auch der Anteil an Senken für die in der Pore vorhandenen gasförmigen Bestandteile.

2. Es kommt $\mathrm{zu}$ dem im vorigen Abschnitt beschriebenen diskontinuierlichen Kornwachstum und damit verbunden zur Entstehung einer hohen intragranularen Porosität.

3. Ausreichende Porenbeweglichkeit führt andererseits zu Porenkoaleszenz in Korngrenzen und Tripelpunkten.

\subsubsection{Wismutoxid und Molybdäntrioxid als Dotierungen}

Insbesondere bei halbleitenden Zinkoxid-Keramiken, wie sie für die Herstellung von Varistoren verwendet werden, wird erfolgreich Wismutoxid als Dotierung zur Stimulation des Kornwachstums eingesetzt $[67,68]$. Aber auch für weichmagnetische Spinell - Ferrite liegen Ergebnisse vor. 
Hierzu gehören die Untersuchungen zu den Systemen Wismutoxid - Kobaltferrit und Wismutoxid - Nickelferrit von Viting und Golubkova [69]. Die Autoren untersuchten das Phasendiagramm für hohe Wismutoxid Konzentrationen (Abbildung 2.12) und finden Eutektika bei 13,5 Mol.\% bzw. 9 Mol\% Ferrit.

Für das System Kobaltferrit-Wismutoxid ist bei $1100^{\circ} \mathrm{C}$ mit einem Ferritanteil in der Flüssigphase von $40 \mathrm{Mol} . \%$ und bei einer typischen Sintertemperatur von $1350{ }^{\circ} \mathrm{C}$ mit einem Anteil von ungefähr $60 \mathrm{Mol}$ \% zu rechnen.

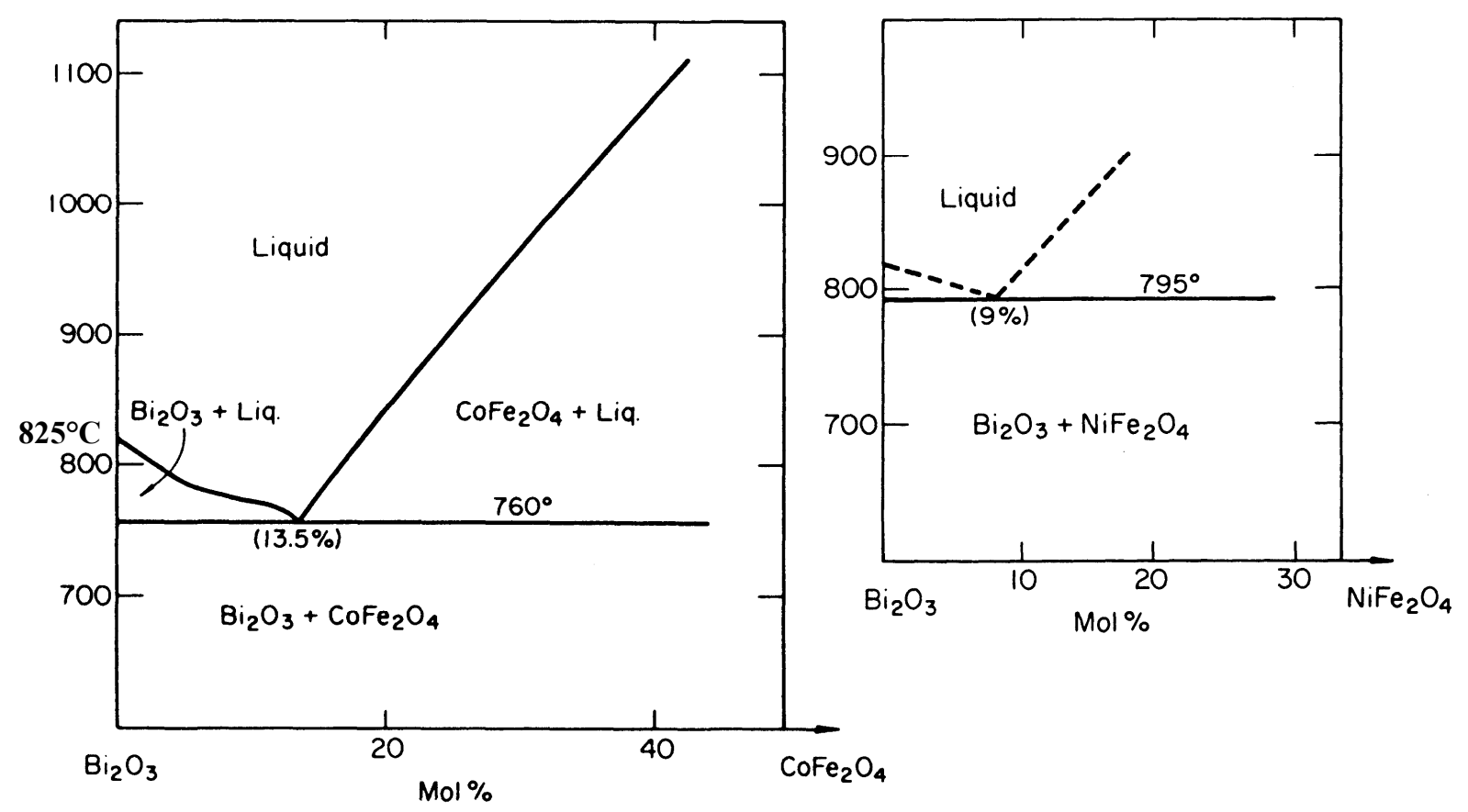

Abbildung 2.12 Phasendiagramm der Systeme Wismutoxid-Kobaltferrit und Wismutoxid-Nickelferrit (nach [69]).

Weitere Veröffentlichungen betreffen den Einsatz von Wismutoxid und Molybdänoxid als Dotierung. Beim Vergleich der Ergebnisse ist jedoch der starke Einfluss des Präparationsprozesses problematisch. Insbesondere bei der Herstellung der keramischen Pulver und beim Sintern dürfen die Einflüsse auch kleinerer Abweichungen auf die erzielten Resultate nicht unterschätzt werden. Die Homogenität der zugegebenen Dotierungen, die Reaktivität des Ferritpulvers sowie die gewählten Sintertemperaturen und Partialdrücke sind in diesem Zusammenhang zu nennen. Trotzdem findet man teilweise ähnliche Aussagen.

Drofenik et al. beschreiben folgendes Verhalten für mit $\mathrm{Bi}_{2} \mathrm{O}_{3}$ dotierte MnZn-Ferrite [70]:

- Bei Dotierungen < 0,05 Gew.\% bleibt die Korngröße konstant bei ca. $12 \mu \mathrm{m}$. 
- Bei Dotierungen zwischen 0,05 Gew.\% bis ca. 0,09 Gew.\% entsteht eine ausgeprägte Duplexstruktur (vgl. Abschnitt 2.2.2).

- Bei Dotierungen $\geq 0,1$ Gew.\% tritt starkes diskontinuierliches Kornwachstum mit hoher intrakristalliner Porosität auf. Es findet sich kein normalgewachsenes Gefüge mehr.

- Bei Dotierungen $\geq 0,3$ Gew.\% finden die Autoren wieder normales Kornwachstum mit Korngrößen von ca. $17 \mu \mathrm{m}$.

In der Patentanmeldung [71] und in [36] werden Änderungen der elektrischen Eigenschaften bei hochpermeablen MnZn-Ferriten durch Zugabe von $\mathrm{Bi}_{2} \mathrm{O}_{3}, \mathrm{MoO}_{3}$ und Kombinationen aus beiden Additiven beschrieben. Bei Zugabe von 0,02 Gew.\% $\mathrm{Bi}_{2} \mathrm{O}_{3}$ erhöht sich die Anfangspermeabilität $\mu_{i}$ von 7200 auf 7800, einhergehend mit mittleren Korngrößen $\bar{X}>50 \mu \mathrm{m}$ und starker intragranularer Porosität. Bei Zugabe von 0,02 Gew.\% $\mathrm{MoO}_{3}$ wird eine Abnahme der Permeabilität auf $\mu_{i}=6900$ und kein nennenswerter Einfluss auf das Kornwachstum angegeben.

Bei den älteren Untersuchungen von Jain et al. wird mit 0,5 Gew.\% vergleichsweise viel Molybdäntrioxid als Zusatz verwendet [72]. Die Autoren beschreiben bei Sinterung in Sauerstoffatmosphäre im Vergleich zur undotierten Probe eine leichte Abnahme der Korngröße.

Die Ergebnisse von Matsuo et al. zur Wirkung von Molybdänoxid beschreiben eine Steigerung der Permeabilität von $\mu_{i} \approx 7500$ auf $\mu_{i} \approx 12000$ bei Zugabe einer verhältnismäßig großen Menge von $0,9 \mathrm{Gew} . \% \mathrm{MoO}_{3}$. Die Korngröße wächst hierbei von ca. 14 auf $22 \mu \mathrm{m}$ und die Porosität nimmt $\mathrm{zu}$, es findet jedoch kein ausgeprägtes diskontinuierliches Kornwachstum statt [73]. Ono et al. beschreiben in [74] für deutlich geringere $\mathrm{MoO}_{3}-$ Dotierungen von $0,06 \mathrm{Gew} \%$ sehr hohe Anfangspermeabilitäten von ca. $\mu_{i} \approx 24000$ bei einer Sintertemperatur von $1400{ }^{\circ} \mathrm{C}$.

\subsection{Grundlagen des Mikrowellensinterns}

In der Praxis ist während des Sinterns immer mit Temperaturinhomogenitäten zu rechnen. Bei der Widerstandsbeheizung muss die Wärme von den verwendeten Heizelementen zum Sintergut gelangen, die Erwärmung des Sintergutes erfolgt entsprechend von außen nach innen. Eine Alternative hierzu stellt die Verwendung von Mikrowellenstrahlung zum Erwärmen des Sintergutes dar. Die Wärme wird durch Wechselwirkung (Kopplung) mit der Strahlung direkt im Sintergut erzeugt. Die Anwendung des kombinierten Verfahrens, der Elektro-Mikrowellen-Hybridsinterung ist 
Teil der durchgeführten Untersuchungen zur Verbesserung der Materialeigenschaften von Ferriten und in Abschnitt 4.2 beschrieben. Im Folgenden wird deshalb eine kurze Einführung in die für das Mikrowellensintern wichtigsten physikalischen Mechanismen gegeben.

\section{Permeabilität, Dielektrizitätszahl und Ausbreitungskonstante}

Die Wechselwirkung eines Körpers mit einem elektromagnetischen Feld wird durch seine Dielektrizitätszahl, seine Permeabilität (vgl. 2.1.4) und seine Leitfähigkeit beschrieben. Dielektrizitätszahl und Permeabilität sind komplexe Größen und sowohl von der Frequenz als auch von der Feldstärke abhängig. Man definiert Real- und Imaginärteil sowie den Verlustwinkel der Dielektrizitätszahl wie folgt (vgl. 3.4.1):

$$
\underline{\mu}=|\underline{\mu}| e^{-i \delta_{\mu}}=\mu^{\prime}-i \mu^{\prime \prime} \text { bzw. } \quad \underline{\varepsilon}=|\underline{\varepsilon}| e^{-i \delta_{\varepsilon}}=\varepsilon^{\prime}-i \varepsilon^{\prime \prime},
$$

mit dem Realteil

$$
\mu^{\prime}=|\underline{\mu}| \cos \delta_{\mu} \quad \text { bzw. } \quad \varepsilon^{\prime}=|\underline{\varepsilon}| \cos \delta_{\varepsilon}
$$

und dem Imaginärteil

$$
\mu^{\prime \prime}=|\underline{\mu}| \sin \delta_{\mu} \quad \text { bzw. } \quad \varepsilon^{\prime \prime}=|\underline{\varepsilon}| \sin \delta_{\varepsilon} .
$$

$\delta_{\mu}$ und $\delta_{\varepsilon}$ sind der magnetische und der elektrische Verlustwinkel. Nicht berücksichtigt ist hier die Wirkung der ohmschen Verluste. Sie lassen sich zusammen mit den dielektrischen Verlusten zu einem effektiven Verlustanteil $\varepsilon_{\text {eff }}^{\prime \prime}$ zusammenfassen:

$$
\underline{\varepsilon}=\varepsilon^{\prime}-i \varepsilon_{e f f}^{\prime \prime}=\varepsilon^{\prime}-i\left(\varepsilon^{\prime \prime}+\frac{\sigma}{\varepsilon_{0} \omega}\right),
$$

( $\sigma=$ Leitfähigkeit in $1 / \Omega \mathrm{m}, \omega=$ Kreisfrequenz). Aus $\underline{\mu}$ und $\underline{\varepsilon}$ berechnet sich die komplexe Ausbreitungskonstante $\underline{\gamma}=\sqrt{\underline{\mu} \underline{\varepsilon}}$, die auch als komplexer Brechungsindex bezeichnet wird. Der dazugehörige Verlustwinkel ergibt sich zu

$$
\delta_{\gamma}=\frac{\delta_{\mu}+\delta_{\varepsilon}}{2}
$$

Die Ausbreitungs- oder Fortpflanzungskonstante $\underline{\gamma}$ beschreibt die Wechselwirkung der elektromagnetischen Welle mit der Materie, also Ausbreitungsgeschwindigkeit, Reflexion, Brechung und Absorption. 


\section{Eindringtiefe und Absorption}

Die in den Festkörper eindringende Mikrowellenstrahlung wechselwirkt mit den vorhandenen magnetischen und elektrischen Momenten. Es kommt zum Ankoppeln des Feldes und insbesondere beim Vorhandensein von Resonanzen zur Energiedissipation. Aus den Maxwellgleichungen ergibt sich für die Ausbreitung einer Welle im Festkörper eine komplexe Wellenzahl $\underline{k}=k \underline{\gamma}$, wobei $k$ die Wellenzahl im Vakuum ist $\left(k=\omega / c=\omega \sqrt{\mu_{0} \varepsilon_{0}}\right)$.

Die Ausbreitungsgleichung einer ebenen Welle wird entsprechend zu:

$$
E=\hat{E} e^{i k \underline{x}-i \omega t}=\hat{E} e^{i k \operatorname{Re}(\underline{\gamma}) x-i \omega t} e^{-k \operatorname{Im}(\underline{\gamma}) x} .
$$

Sie zerfällt in zwei Terme, von denen der erste die Ausbreitung mit der in Materie geänderten Wellenlänge $\lambda^{\prime}$ beschreibt:

$$
\lambda^{\prime}=\frac{\lambda}{\operatorname{Re}(\gamma)}=\frac{\lambda}{\sqrt{|\underline{\varepsilon}||\underline{\mu}|} \cos \delta_{\gamma}} .
$$

Der zweite Term gibt die Dämpfung der Welle wieder. Die Eindringtiefe $d$ ist die Strecke, nach der sich die Feldstärke um einen Faktor 1/e verringert:

$$
d=\frac{1}{k \operatorname{Im}(\gamma)}=\frac{1}{k \sqrt{|\underline{\varepsilon \mid}| \underline{\mu} \mid} \sin \delta_{\gamma}} .
$$

Ein hoher Realteil der Ausbreitungskonstanten verringert also die Wellenlänge, während ein hoher Imaginärteil die Eindringtiefe des Feldes reduziert.

\section{Erwärmung durch Mikrowellenstrahlung und Wärmeleitung}

Das eindringende Feld gibt seine Energie an den Festkörper ab. Die dissipierte Leistung $d P_{\text {diss }}$ hängt von der lokalen magnetischen und elektrischen Feldstärke ab und beträgt für ein Volumen $d V[75,76]$

$$
d P_{d i s s}=\frac{1}{2} \omega\left(\varepsilon_{0} \varepsilon_{e f f}^{\prime \prime}|\vec{E}|^{2}+\mu_{0} \mu_{r}^{\prime \prime}|\vec{H}|^{2}\right) d V
$$

Die so entstandene Wärme wird innerhalb des Festkörpers durch Wärmeleitung weitergegeben. Die Wärmeleitungsgleichung, eine partielle Differentialgleichung zweiter Ordnung, beschreibt diesen Vorgang.

$$
\rho c_{w} \frac{\partial T}{\partial t}=\vec{\nabla} \lambda_{w} \vec{\nabla} T
$$


$\left(\vec{\nabla}=(\partial / \partial x ; \partial / \partial y ; \partial / \partial z), \quad T=\right.$ Temperaturverteilung im Festkörper $[\mathrm{K}], \quad \lambda_{w}=$ Wärmeleitfähigkeit $\left[\mathrm{J} /\left(\begin{array}{lll}\mathrm{K} & \mathrm{s} & \mathrm{m}\end{array}\right)\right] c_{w}=$ Wärmekapazität $[\mathrm{J} /(\mathrm{K} \quad \mathrm{kg})], \rho=$ Dichte des übertragenden Mediums $\left.\left[\mathrm{kg} / \mathrm{m}^{3}\right]\right)$.

Fasst man die Wärmeleitungsgleichung mit dem Quellterm (2.39) zusammen, so erhält man folgenden Zusammenhang:

$$
\frac{1}{2} \omega\left(\varepsilon_{0} \varepsilon_{e f f}^{\prime \prime}|\vec{E}|^{2}+\mu_{0} \mu_{r}^{\prime \prime}|\vec{H}|^{2}\right)=\rho c_{w}\left(\frac{\partial T}{\partial t}\right)-\vec{\nabla}\left(\lambda_{w} \vec{\nabla} T\right) .
$$

Hierbei ist zu beachten, dass sämtliche in der Gleichung vorkommenden Konstanten temperaturabhängig sind. Insbesondere die Verlustanteile von Permeabilität und Dielektrizitätszahl zeigen eine nicht zu vernachlässigende Abhängigkeit. Bei Ferriten kommt es häufig zu einem plötzlichen, starken Anstieg des dielektrischen Verlustanteils mit der Temperatur und damit zur Gefahr der Hotspotbildung. Gleichung 2.41 beschreibt die Wärmeentstehung und Wärmeleitung innerhalb eines Festkörpers oder zwischen Festkörpern im thermischen Kontakt, also in der Praxis zwischen Sintergut und den verwendeten keramischen Trägerplatten bzw. Sinterhilfsmitteln.

Dies gibt die Situation innerhalb eines Ofens nur unvollständig wieder. Hinzu kommt noch der Wärmeaustausch zwischen Oberflächen durch Wärmestrahlung und die Erwärmung durch Konvektion, welche über eine entsprechende Luftumwälzung gezielt für den Temperaturausgleich eingesetzt wird. 


\section{Experimentelle Methoden}

Das Kapitel gibt einen Überblick über die angewandten experimentellen Methoden. In den Abschnitten 3.1 und 3.2 wird auf die Zusammensetzung der Ferritproben und auf die Methoden zur Probenpräparation eingegangen. Abschnitt 3.3 gibt einen Überblick über die verwendete Sintertechnik und beschreibt insbesondere den eingesetzten Elektro Mikrowellen - Hybridsinterofen. Die für die magnetische Charakterisierung und für die Mikrostrukturuntersuchungen angewandten Verfahren werden abschließend in den Abschnitten 3.4 und 3.5 abgehandelt.

\subsection{Zusammensetzung der Ferritproben}

\subsubsection{Hochpermeable MnZn-Ferrite}

Wie in Abschnitt 2.1.4 beschrieben, ist die Permeabilität der Ferrite von der stark temperaturabhängigen magnetokristallinen Anisotropieenergie bestimmt. MnZn-Ferrite zeigen bei Raumtemperatur nur in der Nähe einer Zusammensetzung von 52 Mol.\% $\mathrm{Fe}_{2} \mathrm{O}_{3}, 25 \mathrm{Mol}$ \% MnO und $23 \mathrm{Mol}$ \% $\mathrm{ZnO}$ höchste Permeabilitäten. Das Material ist also leicht überstöchiometrisch und hat bei Raumtemperatur neben einem Nulldurchgang der magnetokristallinen Anisotropieenergie $E_{K}$ auch ein Minimum der magnetostriktiven Energie $E_{M E}$.

Abbildung 3.1 zeigt die Temperaturabhängigkeit des sekundären Permeabilitätsmaximums für eine Versuchsreihe mit variierendem Gehalt an Eisenoxid und konstantem MnO-Gehalt von 25,5 Mol.\% (x Mol.\% $\mathrm{Fe}_{2} \mathrm{O}_{3}, 25,5 \mathrm{Mol} \% \mathrm{MnO}, 74,25$ - x Mol.\% ZnO, 0,25 Mol.\% $\mathrm{TiO}_{2}$ ).

Die zugegebene $\mathrm{TiO}_{2}$-Dotierung beeinflusst über Umladungsprozesse das $\mathrm{Fe}^{2+} / \mathrm{Fe}^{3+}$ Gleichgewicht und die Breite des SPM. Das $\mathrm{Ti}^{4+}$-Ion wird dabei im Kristallgitter bevorzugt auf B-Plätzen eingebaut [77]. 


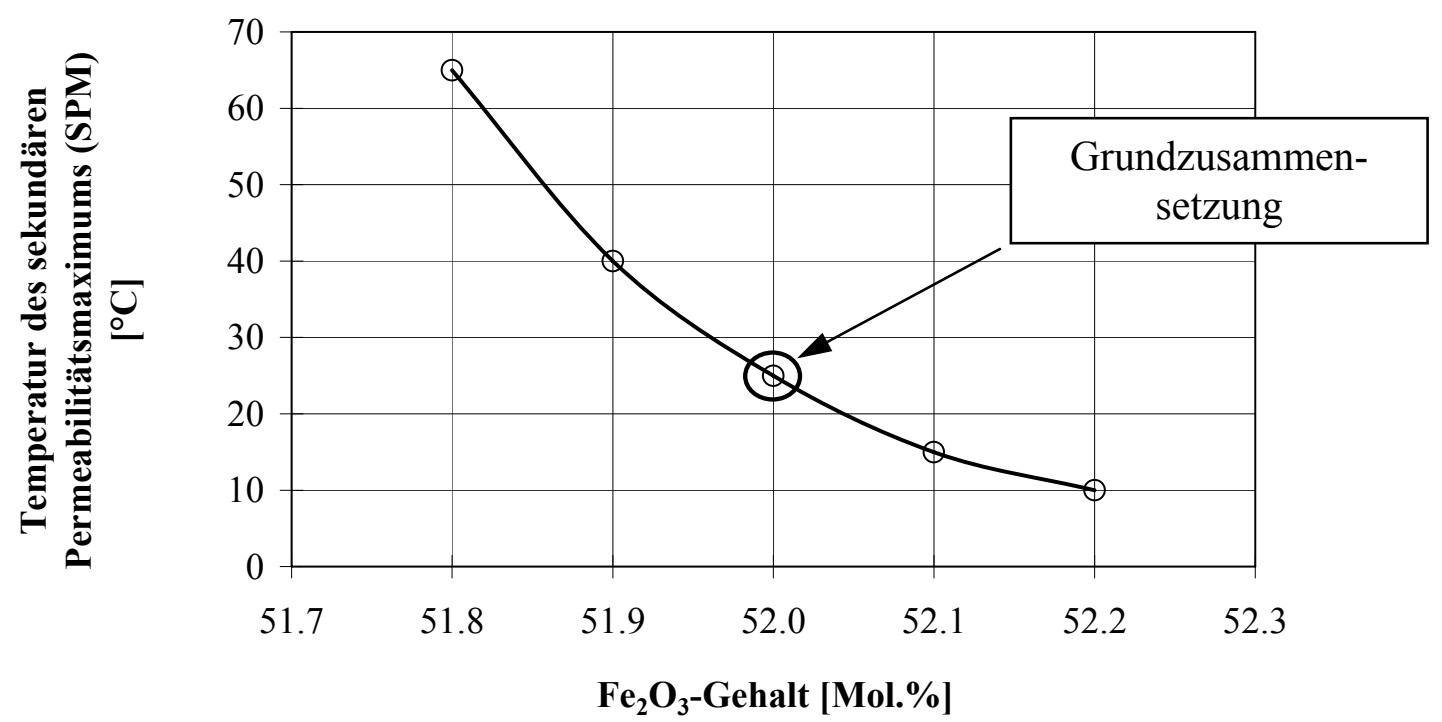

Abbildung 3.1 Auswahl des Eisengehaltes für die Grundzusammensetzung. Mit zunehmendem Eisenoxidgehalt verschiebt sich das Sekundäre Permeabilitätsmaximium zu niedrigeren Temperaturen.

\section{Grundmischung HP1}

Aufgrund dieser Voruntersuchungen wurde die Grundmischung HP1 für die Untersuchungen in Abschnitt 4.1 gewählt. Sie besteht aus

$$
\begin{array}{ll}
\text { 52,05 Mol.\% } & \mathrm{Fe}_{2} \mathrm{O}_{3}, \\
\text { 25,50 Mol.\% } & \mathrm{MnO}\left(\text { Zugabe als } \mathrm{Mn}_{3} \mathrm{O}_{4}\right), \\
\text { 22,20 Mol.\% } & \mathrm{ZnO}, \\
\text { 0,25 Mol.\% } & \mathrm{TiO}_{2} .
\end{array}
$$

\section{$\underline{\text { Dotierungen }}$}

Zur Untersuchung des Flüssigphasensinterns wurden Mischungen mit unterschiedlichen Anteilen an niedrigschmelzenden Additiven hergestellt. Es handelt sich zum einen um Wismutoxid $\mathrm{Bi}_{2} \mathrm{O}_{3}$ mit einem Schmelzpunkt von $825^{\circ} \mathrm{C}$, zum anderen um Molybdäntrioxid $\mathrm{MoO}_{3}$ mit einem Schmelzpunkt von $795^{\circ} \mathrm{C}$. Eine Zusammenstellung der gefertigten Mischungen sowie der im Folgenden verwendeten Kennzeichnungen findet sich in Tabelle 3.1. 


\begin{tabular}{c|c|c|l}
\hline $\begin{array}{c}\text { Grund- } \\
\text { mischung }\end{array}$ & Dotierung & $\begin{array}{c}\text { Menge } \\
{[\mathrm{Gew} . \%]}\end{array}$ & Kennzeichnung \\
\hline $\mathrm{HP} 1$ & keine & - & $\mathrm{HP} 1$ \\
\hline $\mathrm{HP} 1$ & $\mathrm{Bi}_{2} \mathrm{O}_{3}$ & 0,02 & HP1_Bi2 \\
\hline $\mathrm{HP} 1$ & $\mathrm{Bi}_{2} \mathrm{O}_{3}$ & 0,03 & HP1_Bi3 \\
\hline $\mathrm{HP} 1$ & $\mathrm{Bi}_{2} \mathrm{O}_{3}$ & 0,04 & HP1_Bi4 \\
\hline $\mathrm{HP} 1$ & $\mathrm{Bi}_{2} \mathrm{O}_{3}$ & 0,06 & HP1_Bi6 \\
\hline $\mathrm{HP} 1$ & $\mathrm{Bi}_{2} \mathrm{O}_{3}$ & 0,08 & HP1_Bi8 \\
\hline $\mathrm{HP} 1$ & $\mathrm{MoO}_{3}$ & 0,02 & HP1_Mo2 \\
\hline $\mathrm{HP} 1$ & $\mathrm{MoO}_{3}$ & 0,04 & HP1_Mo4 \\
\hline $\mathrm{HP} 1$ & $\mathrm{MoO}_{3}$ & 0,06 & HP1_Mo6 \\
\hline
\end{tabular}

Tabelle 3.1 Übersicht über die gefertigten Ferritproben und deren Zusammensetzung.

\subsubsection{Ferrit für die Leistungsübertragung und NiZn-Ferrit}

Für die Untersuchungen mit dem Elektro-Mikrowellen-Hybridsinterofen (Abschnitt 4.2) wurden Materialien gewählt, bei denen mit einer Eigenschaftsverbesserung durch die erwartete Gefügefeinung zu rechnen ist.

\section{Grundmischung L1}

Bei der Zusammensetzung L1 handelt es sich um MnZn - Ferrit für Leistungsanwendungen bei höheren Frequenzen. Die Zusammensetzung ist optimiert für hohe Sättigungsmagnetisierungen und niedrige Ummagnetisierungsverluste:

$52,80 \mathrm{Mol} . \% \mathrm{Fe}_{2} \mathrm{O}_{3}$,

36,90 Mol.\% $\mathrm{MnO}\left(\right.$ Zugabe als $\left.\mathrm{Mn}_{3} \mathrm{O}_{4}\right)$,

10,30 Mol.\% ZnO . 


\section{Grundmischung Ni1}

Für Sinterungen unter ungeregelter Luft - Atmosphäre eignen sich MnZn-Ferrite nicht. Das Mn-Ion ändert beim Abkühlen seine Wertigkeit und die Spinellphase zerfällt (vgl. Abschnitt 3.2). Besser geeignet sind hier NiZn-Ferrite mit dem gegen Oxidation stabilen zweiwertigen $\mathrm{Ni}^{2+}$-Ion:

$$
\begin{aligned}
& \text { 49,60 Mol.\% } \mathrm{Fe}_{2} \mathrm{O}_{3}, \\
& \text { 16,80 Mol.\% } \mathrm{NiO}, \\
& \text { 33,60 Mol.\% } \mathrm{ZnO} .
\end{aligned}
$$

\subsection{3 Überprüfung der Zusammensetzung mittels RFA}

Der Gehalt an $\mathrm{Bi}_{2} \mathrm{O}_{3}$ wurde mittels Röntgenfluoreszenzanalyse (RFA) bestimmt. Zum Einsatz kam hierbei ein RFA-Gerät vom Typ Philips PW 1404.

Zur Präparation werden die Proben in einem Mörser zerrieben und auf mehreren Brennern, bei bis auf $1000^{\circ} \mathrm{C}$ steigenden Temperaturen, zusammen mit einem Gemisch aus Lithiumtetraborat und Aluminiumoxid erwärmt, aufgeschmolzen, gelöst und homogenisiert (Zusammensetzung: 0,2g Probenmaterial, 3,6g $\mathrm{Li}_{2} \mathrm{~B}_{4} \mathrm{O}_{7}, 0,4 \mathrm{~g} \mathrm{Al} \mathrm{Al}_{2}$ ). Aus der Glasschmelze gießt man Tabletten und analysiert diese mittels eines halbquantitativen Verfahrens. Der relative Fehler ist aufgrund der niedrigen Konzentrationen verhältnismäßig hoch und beträgt $15 \%$.

Das Einschmelzen hat den Vorteil einer höheren Homogenität und Reproduzierbarkeit, verdünnt aber das Probenmaterial nochmals stark.

\subsection{Herstellung von weichmagnetischen Ferriten}

Die zur Herstellung der Formkörper angewandte Methode ist in Abbildung 3.2 skizziert und in [16] detailliert beschrieben. Der Prozess folgt weitestgehend einer Verfahrensvariante, die auch im industriellen Maßstab durchgeführt wird. Es handelt sich um ein Nassverfahren mit oxidischen Ausgangsprodukten. 


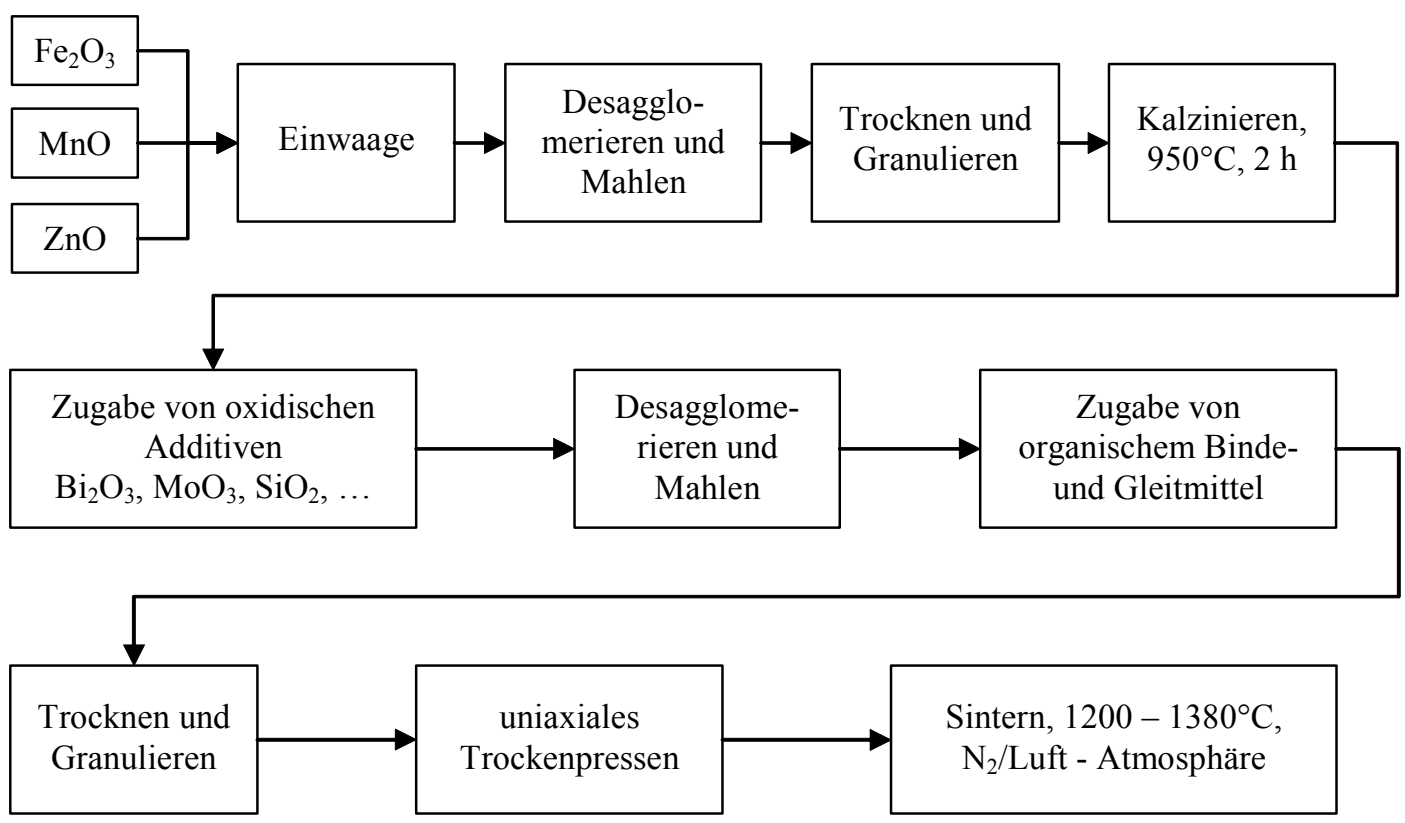

Abbildung 3.2 Schematische Darstellung des Herstellungsprozesses für keramische Formkörper, Nassverfahren unter Verwendung oxidischer Ausgangsprodukte.

\subsubsection{Pulverpräparation und Pressen von Grünkörpern}

\section{Rohstoffe und Einwaage}

Als Rohstoffe dienen Metalloxide mit technischer Reinheit (Tabelle 3.2). Die vorhandenen Verunreinigungen sind, wo möglich, bei der Berechnung der Einwaage berücksichtigt.

\begin{tabular}{l|c|c|c|c|l}
\hline Rohstoff & \multicolumn{2}{|c|}{ Reinheit [Gew.\%] } & \multicolumn{3}{|c}{ Verunreinigungen } \\
\hline Eisenoxid & $\mathrm{Fe}_{2} \mathrm{O}_{3}$ & 99,5 & $70 \mathrm{ppm} \mathrm{SiO}_{2}$ & $80 \mathrm{ppm} \mathrm{CaO}$ & $0,25 \% \mathrm{MnO}$ \\
\hline Manganoxid $^{[i]}$ & $\mathrm{MnO}$ & 92,0 & $30 \mathrm{ppm} \mathrm{SiO}{ }_{2}$ & $10 \mathrm{ppm} \mathrm{CaO}$ & \\
\hline Zinkoxid & $\mathrm{ZnO}$ & 99,8 & $2 \mathrm{ppm} \mathrm{Pb}$ & & \\
\hline Titandioxid & $\mathrm{TiO}_{2}$ & 99,0 & & & \\
\hline Nickeloxid & $\mathrm{NiO}$ & 98,0 & $100 \mathrm{ppm} \mathrm{Fe}$ & $10 \mathrm{ppm} \mathrm{Cu}$ & \\
\hline
\end{tabular}

Tabelle 3.2 Übersicht über die verwendeten Rohstoffe. Die niedrige Reinheit des Manganoxids kommt durch die Verwendung von $\mathrm{Mn}_{3} \mathrm{O}_{4}$ zustande.

[i] Es handelt sich um ein Gemisch unterschiedlicher Manganoxide mit einem hohen Anteil an $\mathrm{Mn}_{3} \mathrm{O}_{4}$. 


\section{Desagglomerieren und Mahlen}

Die Mischungen werden mit Wasser und Stahlkugeln in Polyethylenflaschen über $24 \mathrm{~h}$ gemahlen. Ziel dieses Prozessschrittes ist zum einen eine Homogenisierung der Rohstoffe, zum anderen eine Zerkleinerung der aus Primärpartikeln gebildeten, relativ lose gebundenen Rohstoffagglomerate. Ein Zermahlen der Primärpartikel selbst findet nicht statt. Ein Ansatz besteht aus:

700 g Rohoxide,

1,5 kg Stahlkugeln, $700 \mathrm{ml} \mathrm{H}_{2} \mathrm{O}$,

$3 \mathrm{ml}$ Dispergiermittel (Ammoniumsalz einer polymeren Carbonsäure).

\section{$\underline{\text { Kalzinieren }}$}

Nach dem Mahlen werden die Mahlkugeln entfernt und das Wasser bei $150{ }^{\circ} \mathrm{C}$ abgedampft. Das verbleibende Material wird durch einen Sieb gerieben, in einem Kammerofen bei $950{ }^{\circ} \mathrm{C}$ für eine Stunde kalziniert und langsam im Ofen abgekühlt. Während der Kalzination bilden sich bei Temperaturen $>600{ }^{\circ} \mathrm{C}$ Zinkferrit $\left(\mathrm{ZnFe}_{2} \mathrm{O}_{4}\right)$ und durch eindiffundieren von Mn-Ionen ab $800{ }^{\circ} \mathrm{C}$ auch MnZn-Ferrit [78]. Die so entstandene Spinellphase ist jedoch metastabil und zerfällt beim Abkühlen durch Oxidation.

Anders verhalten sich hier NiZn-Ferrite, bei denen die Spinellphase aufgrund des gegen Oxidation stabilen $\mathrm{Ni}^{2+}$-Ions nicht wieder zerfällt.

Zweck der Kalzination ist eine weitere Homogenisierung des Ferritpulvers.

\section{$\underline{\text { Granulieren }}$}

Man gibt nun die Dotierung der kalzinierten Grundmischung zu und desagglomeriert ein zweites Mal nach dem oben beschriebenen Verfahren mittels der Kugelmühle. Danach durchmischt man das entstandene Pulver in einem Knetgerät mit einer wässrigen Polyvinylalkohol-Lösung (PVA) und dampft gleichzeitig bei $120^{\circ} \mathrm{C}$ einen Großteil des Wassers ab. Der in einer Menge von 0,85 Gew.\% zugegebene Polyvinylalkohol wirkt als Bindemittel und sorgt sowohl für die Stabilität des Pressgranulats als auch des verdichteten Grünkörpers. Bei ca. $40^{\circ} \mathrm{C}$ erfolgt die Einstellung einer Restfeuchte von 0,3 Gew.\%. Die eigentliche Granulation erfolgt, indem die entstandenen Bruchstücke durch ein Sieb der Maschenweite 0,35 mm gerieben werden (Siebgranulation). 


\section{Pressen der Grünkörper}

Um die beim anschließenden Pressen der Grünkörper auftretende Reibung zu reduzieren werden 0,2 Gew.\% Zn-Stearat untergemengt. Das Pressen erfolgt bei Raumtemperatur einachsig, bidirektional mit einer mechanischen Exzenterpresse auf eine Dichte von $3,0 \pm 0,1 \mathrm{~g} / \mathrm{cm}^{3}$. Um die Charakterisierung der magnetischen Eigenschaften einfach $\mathrm{zu}$ gestalten werden Ringe mit den Abmessungen $d_{a} \times d_{i} \times h=19 \times 12 \times 6$ mm$^{3}$ angefertigt. $\left(\mathrm{d}_{\mathrm{a}}=\varnothing_{\text {außen }}, \mathrm{d}_{\mathrm{i}}=\varnothing_{\text {innen }}, \mathrm{h}=\right.$ Höhe des Ringes $)$.

\subsubsection{Sintern von MnZn-Ferriten}

Im Gegensatz $\mathrm{zu}$ anderen keramischen Materialien wie beispielsweise $\mathrm{Al}_{2} \mathrm{O}_{3}$ oder $\mathrm{SiC}$ bildet sich bei den MnZn-Ferriten die gewünschte Festkörperphase erst während des Sintervorgangs über mehrere Reaktionen aus den weitestgehend unmagnetischen Vorprodukten. Gleichzeitig findet die typische Verdichtung statt, und aus dem offenporigen Grünling mit einem Porenvolumen von 40\% und einer Dichte von ca. $3,1 \mathrm{~g} / \mathrm{cm}^{3}$ entsteht ein kompaktierter, polykristalliner Festkörper mit einer geschlossenen Restporosität von $5-10 \%$. Die lineare Schrumpfung beträgt ca. $20 \%$.

Eine typische Sinterkurve für hochpermeable MnZn-Ferrite zeigt Abbildung 3.3. Auf die einzelnen Phasen wird im Folgenden eingegangen.

\section{Entbinderungsphase}

Im Temperaturbereich unter $450{ }^{\circ} \mathrm{C}$ zersetzen sich die organischen Additive und gasen aus dem Ferritkern aus. Dieser Vorgang wird auch als Entbindern bezeichnet. Zur Vermeidung von Rissen und anderen Beschädigungen darf die Aufheizrate in diesem Bereich nicht zu hoch gewählt werden. Raten von $2 \mathrm{~K} / \mathrm{min}$ haben sich als unkritisch erwiesen.

Lässt sich die Entbinderungsphase nicht im eigentlichen Sinterofen ausführen, z.B. bei Verwendung des Mikrowellenofens (siehe 3.3.2), so wird sie extern in einem widerstandbeheizten Ofen durchgeführt. 


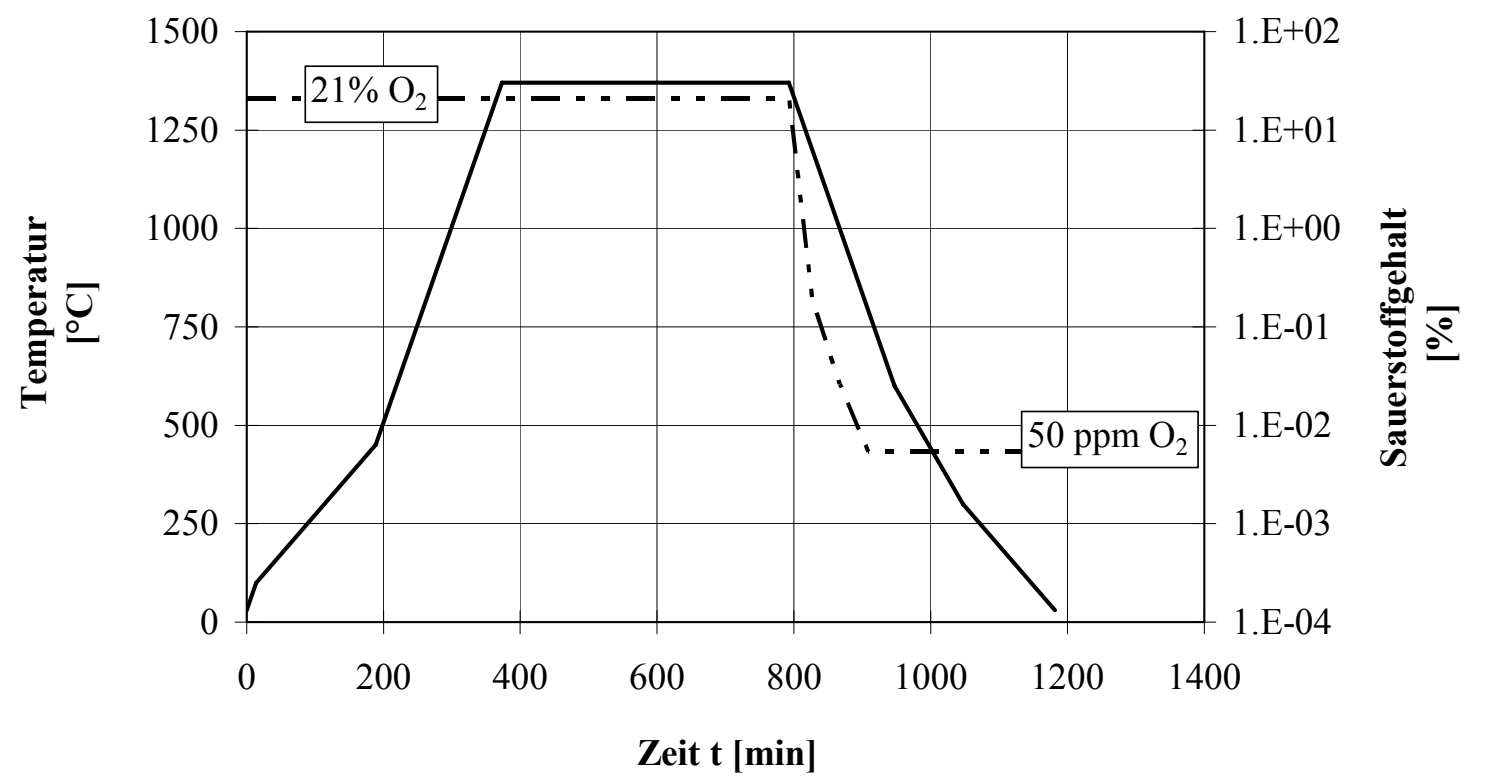

\section{Abbildung 3.3 Temperaturprofil zum Sintern von MnZn-Ferriten. \\ \begin{tabular}{l} 
Temperaturverlauf beim Sintern \\
\hline$\ldots-$ Verlauf der Sauerstoffatmosphäre
\end{tabular}}

Aufheizen und Phasenbildung

Bis zum Erreichen der Sintertemperatur wird das Sintergut normalerweise mit Raten $\leq 7 \mathrm{~K} /$ min erwärmt. Während des Aufheizens von MnZn-Ferriten finden eine Reihe von Phasenbildungsreaktionen statt, die z.B. in [79] und [80] beschrieben sind. Am Ende der Aufheizphase liegt die Spinellphase vollständig vor und der Ferrit hat bereits mehr als $85 \%$ der theoretischen Enddichte erreicht.

\section{Isotherme Haltephase}

Nach Erreichen der Sintertemperatur von 1150 bis $1380{ }^{\circ} \mathrm{C}$ beginnt die Haltephase, die zwar auch einen Beitrag zur Verdichtung des Ferrits leistet, im Wesentlichen aber durch den Prozess des Kornwachstums geprägt ist. In Abhängigkeit von der gewünschten Korngröße und dem vorherrschenden Diffusionsmechanismus (siehe Abschnitt 2.2) kann die Haltezeit zwischen 30 Minuten und 12 h liegen.

\section{Abkühlphase}

Da bei MnZn-Ferriten die Spinellphase unter Luftatmosphäre nur bei Temperaturen über $1000{ }^{\circ} \mathrm{C}$ thermodynamisch stabil ist, bilden sich beim Abkühlen unter Luft durch Oxidation unerwünschte Hämatit-Ausscheidungen. Das Abkühlen muss deshalb geregelt unter reduzierender Stickstoff/Luft-Atmosphäre erfolgen. 
Nach Blank [81] lässt sich die Temperaturabhängigkeit des Sauerstoff-Gleichgewichtspartialdrucks für jede Zusammensetzung und jeden Sauerstoffparameter ${ }^{[i]}$ durch zwei geeignete Konstanten (Blanksche Konstanten) darstellen [82]:

$$
\begin{aligned}
\log \left(C_{O 2}\right)=c_{1} & -\frac{c_{2}}{T}, \\
c_{1}, c_{2} & =\text { Blanksche Gleichgewichtskonstanten, } \\
T & =\text { Temperatur }[\mathrm{K}], \\
C_{O 2} & =\text { Sauerstoffkonzentration }[\%] .
\end{aligned}
$$

Beim Abkühlen unter die Curietemperatur des Materials kommt es zur Entstehung der magnetischen Domänenstruktur. Aufgrund der magnetischen Desakkommodation (vgl. Abschnitt 2.1.2) führt man elektrische und magnetische Messungen frühestens $48 \mathrm{~h}$ nach Beendigung des Sinterprozesses durch [83].

\subsection{Sintertechnik}

Traditionell werden für die industrielle Herstellung von Ferriten widerstandsbeheizte Hubherd- oder Kanalöfen eingesetzt. Erstere bieten aufgrund der geschlossenen Sinterkammer die Möglichkeit einer optimalen Atmosphärenregelung und erlauben das Absenken des Sauerstoffgehalts auf unter $50 \mathrm{ppm} \mathrm{O}_{2}$. Hierdurch sind sie vor allem für MnZn-Ferrite hoher Qualität von Interesse. Letztere haben aufgrund des relativ kleinen Kanalquerschnitts den Vorteil sehr homogener Sinterbedingungen, setzen aber konstruktionsbedingt bei der Temperatur- und Atmosphärenregelung Grenzen. Im Allgemeinen liegen die möglichen Aufheizraten bei maximal 5-7 K/min.

\subsubsection{Laborofen mit Widerstandsbeheizung}

Mit dem verwendeten relativ großvolumigen widerstandsbeheizten Laborofen lassen sich Aufheizraten von maximal $10 \mathrm{~K} / \mathrm{min}$ realisieren. Der Ofen wird vollautomatisch betrieben und erlaubt eine präzise Steuerung der Sinteratmosphäre. Der Sauerstoffpartialdruck lässt sich bis auf ca. $50 \mathrm{ppm}$ absenken. Um einen Einfluss möglicher Temperaturinhomogenitäten innerhalb des Ofenraums auszugleichen, wurden

[i] Der Sauerstoffparameter $\gamma$ gibt den Oxidationsgrad des Ferrits an. Der stöchiometrische Ferrit $\mathrm{A}^{2+} \mathrm{B}^{3+}{ }_{2} \mathrm{O}^{2-}{ }_{4}$ mit den zweiwertigen Kationen $\mathrm{A}^{2+}=\mathrm{Fe}^{2+}, \mathrm{Mn}^{2+}, \mathrm{Zn}^{2+}, \ldots$ und den dreiwertigen Kationen $\mathrm{B}^{3+}=\mathrm{Fe}^{3+}, \mathrm{Mn}^{3+}$ geht bei Erhaltung der Ladungsneutralität und unter Bildung von Kationenleerstellen der Konzentration $\mathrm{C}_{\mathrm{v} \text { kat }}=3 / 4 \gamma$ über in $\mathrm{A}^{2+}{ }_{1-2 \gamma} \mathrm{B}^{3+}{ }_{2+2 \gamma} \mathrm{O}^{2-}{ }_{4+\gamma}$. 
die Proben immer an der gleichen Position innerhalb des Ofens gesintert. Die wichtigsten Eigenschaften des Laborofens sind in Tabelle 3.3 wiedergegeben.

\begin{tabular}{l|l}
\hline $\begin{array}{l}\text { Abmessungen des } \\
\text { Ofenraumes: }\end{array}$ & $1050 \times 750 \times 800 \mathrm{~mm}^{3}$ \\
\hline Ofenvolumen: & $0,63 \mathrm{~m}^{3}$ \\
\hline Heizung: & $\begin{array}{l}\text { Heizleistung P }=4 \times 25 \mathrm{~kW} \text { (vier voneinander } \\
\text { unabhängig geregelte Heizzonen, SiC-Heizstäbe) }\end{array}$ \\
\hline Maximaltemperatur: & $1450{ }^{\circ} \mathrm{C}$ \\
\hline Atmosphärenregelung: & $\begin{array}{l}\text { Die Einstellung des Luft- / Stickstoffgemisches erfolgt } \\
\text { über Mikroprozessorgesteuerte Massendurchflussregler. }\end{array}$ \\
\hline Einblasmengen: & $\begin{array}{l}\text { Stickstoff: } 0-21 \mathrm{~m} / \mathrm{h} \\
\text { Pressluft: } 0-1,2 \mathrm{~m} / \mathrm{h}\end{array}$ \\
\hline Aufheizgeschwindigkeit: & maximal $10 \mathrm{~K} / \mathrm{min}$ \\
\hline Regelung: & $\begin{array}{l}\text { Die Temperatur und der Sauerstoffpartialdruck werden } \\
\text { während der Sinterung kontinuierlich gemessen und } \\
\text { geregelt. }\end{array}$ \\
\hline
\end{tabular}

Tabelle 3.3 Charakteristik des verwendeten widerstandsbeheizten Laborofens.

\subsubsection{Elektro - Mikrowellen - Hybridsintertechnik}

Im Rahmen eines von der Arbeitsgemeinschaft industrieller Forschungsvereinigungen Otto von Guericke geförderten Projektes (AiF Nr: 12072B/2) wurde an der TUBergakademie Freiberg ein Elektro-Mikrowellen-Hybridsinterofen aufgebaut und mit ihm Sinterversuche durchgeführt. Der Ofen besteht aus einer Mikrowellenkammer, in die Mikrowellenstrahlung direkt über einen Hohlleiter eingekoppelt wird. Das in der Kammer befindliche Isolationsmaterial $\left(\mathrm{Al}_{2} \mathrm{O}_{3}\right.$-Fasermaterial KVS 184) ist weitestgehend mikrowellentransparent und bildet die eigentliche Sinterkammer (Abbildung 3.4).

Aufgrund der kompakten Bauweise lassen sich ungewöhnlich hohe Aufheizraten von bis $\mathrm{zu} 50 \mathrm{~K} / \mathrm{min}$ realisieren.

Weitere Charakteristiken sind in Tabelle 3.4 zusammengefasst. 


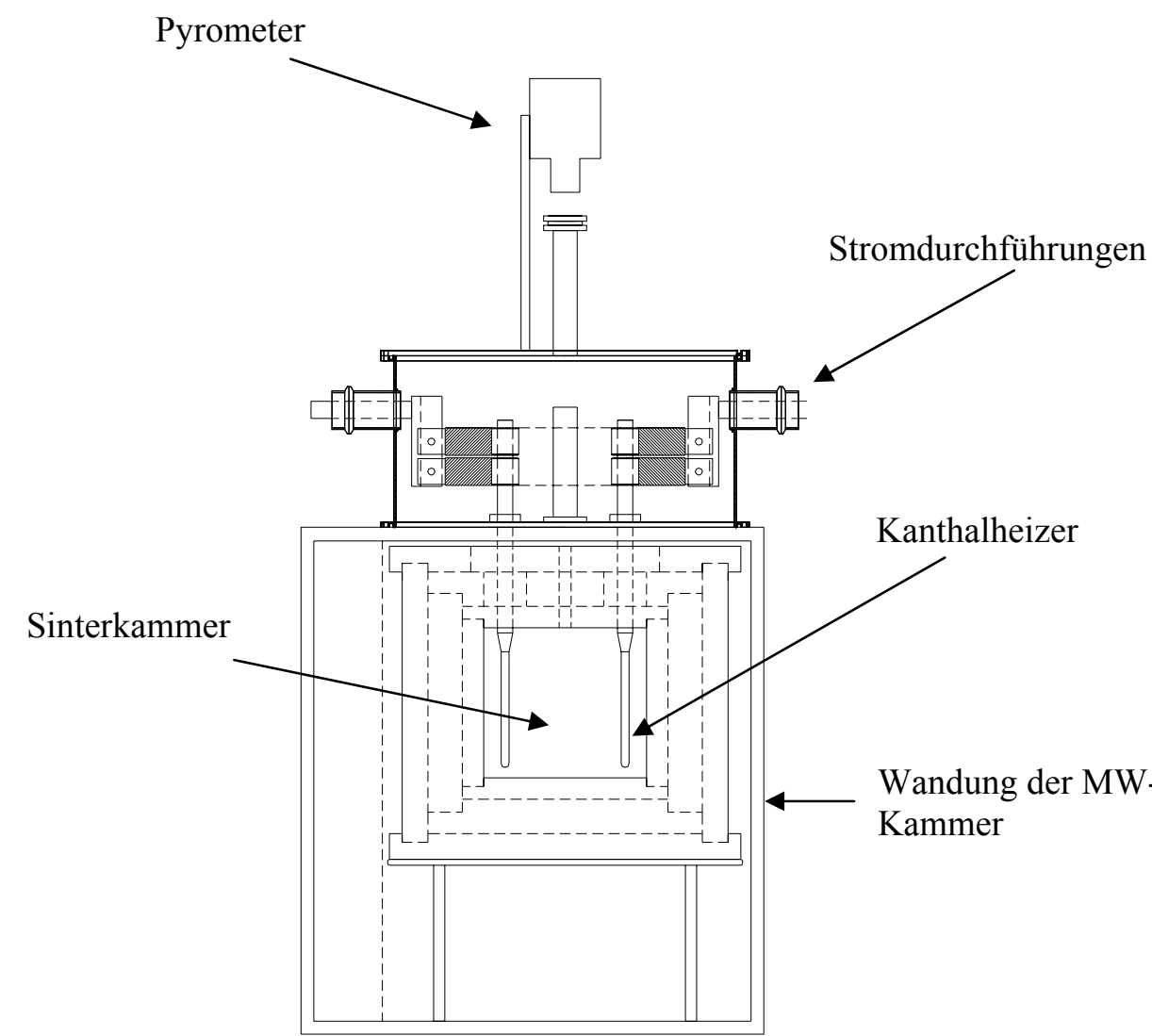

Abbildung 3.4 Schematischer Aufbau des Elektro-Mikrowellen-Hybridsinterofens. Nicht gezeigt sind die Anschlüsse zum Gasaustausch und der Wellenleiter zum Einspeisen der Mikrowellenenergie [84].

\begin{tabular}{l|l}
\hline Abmessungen: & $\begin{array}{l}\text { MW-Kammer: } 380 \times 480 \times 380 \mathrm{~mm}^{3} \\
\text { Sinterkammer: } 100 \times 100 \times 100 \mathrm{~mm}^{3}\end{array}$ \\
\hline Ofenvolumen: & $1000 \mathrm{~cm}^{3}$ \\
\hline Heizung: & $\begin{array}{l}\text { Widerstandsbeheizung }- \text { Kantalheizelemente mit } \\
3 \mathrm{~kW} \text { Heizleistung } \\
\text { Mikrowellenheizung }-6 \mathrm{~kW}, \text { Frequenz } 2,45 \mathrm{GHz}, \\
\text { Regelung von } 10-100 \%\end{array}$ \\
\hline Maximaltemperatur & $1650{ }^{\circ} \mathrm{C}$ \\
\hline Atmosphärenregelung: & $\begin{array}{l}\text { Einstellung des Luft/Stickstoffgemisches über } \\
\text { manuell regelbare Schwebekörperdurchflussmesser. }\end{array}$ \\
\hline Aufheizgeschwindigkeit: & max. 50 K/min \\
\hline Regelung: & $\begin{array}{l}\text { Die Temperatur wird geregelt, die Einstellung des } \\
\text { Sauerstoffpartialdruckes erfolgt manuell. }\end{array}$ \\
\hline
\end{tabular}

Tabelle 3.4 Charakteristik des Elektro-Mikrowellen-Hybridsinterofens 


\subsection{Messung der magnetischen Eigenschaften}

\subsubsection{Messung der komplexen Permeabilität}

Die Messung erfolgt an einem gleichmäßig mit 10 Windungen Litzendraht bewickelten ringförmigen Formkörper mit den Abmessungen $d_{a} \mathrm{x} d_{i} \mathrm{x} h$ (vgl. 3.2.1). Die auftretenden Felder $H$ und $B$ sind homogen, Streufelder können vernachlässigt werden.

Das Verfahren arbeitet mit einer Maxwell-Wien-Brückenschaltung mit automatischem Nullabgleich, bei der über Abstimmung eines parallelen R-C-Gliedes Induktivität und Widerstand bzw. Impedanz $|Z|$ und Phase zwischen Strom und Spannung im Prüfzweig bestimmt werden [85]. Im Frequenzbereich von 10 bis $1000 \mathrm{kHz}$ kommt hierfür ein Impedance - Analyzer HP 4277A der Firma Hewlett Packard und bei Frequenzen größer $1 \mathrm{MHz}$ ein Gerät HP $4191 \mathrm{~A}$ desselben Herstellers zum Einsatz.

Für das Ersatzschaltbild einer Serienschaltung von Induktivität und ohmschem Widerstand gilt $Z=R+i \omega L$. Die Phasenlage wird durch den Verlustwinkel $\delta$ mit $\tan \delta=\frac{R}{\omega L}$ angegeben. Es gilt dann für den Wirkwiderstand $R=|Z| \sin \delta$ und für den Blindwiderstand $\omega L=|Z| \cos \delta$ (Abbildung 3.5).
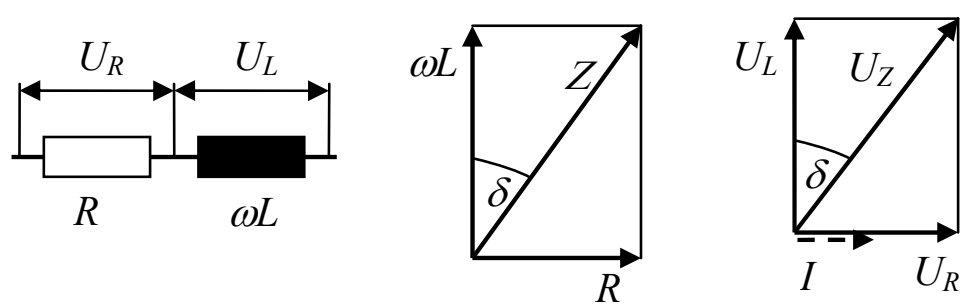

Abbildung 3.5 Serielles Ersatzschaltbild für eine Induktivität. Zeigerdiagramme für den komplexen Widerstand bzw. für Spannung und Strom.

Als geometrieunabhängige Materialkenngröße berechnet man die dimensionslose komplexe Permeabilität $\underline{\mu}=\mu^{\prime}-i \mu^{\prime \prime}$ mit dem Realteil $\mu^{\prime}$ und dem Imaginärteil - $\mu^{\prime \prime}$ :

$$
\begin{aligned}
& \mu^{\prime}=L \frac{2 \pi}{N^{2} \mu_{0} h \ln \left(d_{a} / d_{i}\right)}, \\
& \mu^{\prime \prime}=\frac{R}{\omega} \frac{2 \pi}{N^{2} \mu_{0} h \ln \left(d_{a} / d_{i}\right)}
\end{aligned}
$$

( $L=$ Induktivität der Spule $[\mathrm{H}], R=$ ohmscher Widerstand $[\Omega], N=$ Windungszahl der Spule, $\omega=2 \pi f=$ Kreisfrequenz $[1 / \mathrm{s}], \quad \mu_{0}=4 \pi \cdot 10^{-7} \mathrm{Vs} / \mathrm{Am}=$ magnetische Feldkonstante). 
Das Verhältnis von Imaginärteil zu Realteil bestimmt der Verlustwinkel $\delta^{[\mathrm{i}]}$ bzw. die Güte $Q$ gemäß $\frac{\mu^{\prime \prime}}{\mu^{\prime}}=\tan \delta=\frac{1}{Q}=\frac{R}{\omega L}$.

Bei den Messungen muss nach DIN IEC 60401 eine Induktion $\hat{B} \leq 0,25 m T$ eingehalten werden. Nur so sind die bei höheren Aussteuerungen auftretenden nichtlinearen Verzerrungen im Strom- bzw. Spannungsverlauf vermeidbar.

\subsubsection{Messung der Ummagnetisierungsverluste}

Bei größeren Aussteuerungen reicht die Kenntnis von Phase und Impedanz nicht mehr für die Charakterisierung der Induktivität aus. Der lineare Zusammenhang zwischen Strom und Spannung verschwindet und die Hysterese des Magnetmaterials verursacht merkliche Nichtlinearitäten.

Die Messung der Ummagnetisierungsverluste bei Induktionen $\geq 25 \mathrm{mT}$ erfolgt mittels eines B-H-Analyzers SY-8216 der Firma Iwatsu, der über A/D-Wandler eine Zeitauflösung des Strom- bzw. Spannungssignals ermöglicht. Das Messprinzip ist aus Abbildung 3.6 erkennbar.

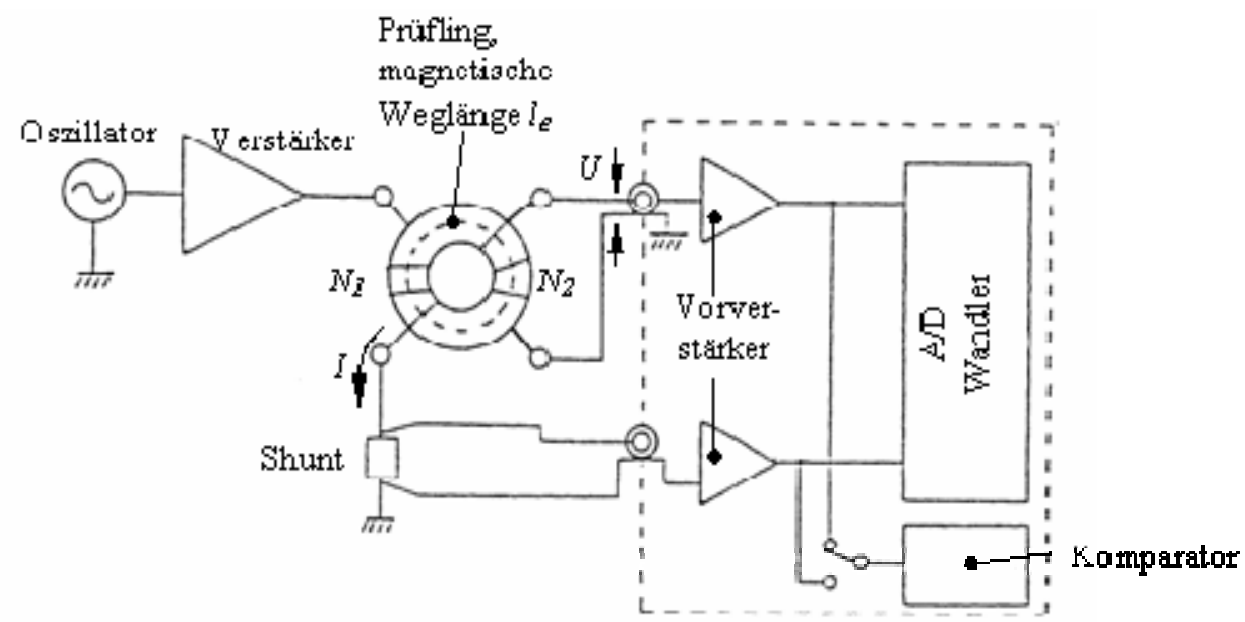

Abbildung 3.6 Messprinzip für die Verlustleistungsmessung (Vierpolmessung). Der durch die Primärwicklung $N_{l}$ fließende Strom $I$ wird mithilfe eines hochpräzisen ohmschen Widerstandes (Shunt) in ein Spannungssignal umgesetzt. Strom und induzierte Spannung werden mit hoher Abtastrate durch einen A/D Wandler erfasst.

[i] Der Verlustwinkel $\delta$ entspricht dem in Abschnitt 2.3 definierten $\delta_{\mu}$. Da hier eine Verwechslung mit $\delta_{\varepsilon}$ ausgeschlossen ist, wird auf den Index verzichtet. 
Die Ummagnetisierungsverluste $P$ bei der Arbeitsfrequenz $f$ werden aus den elektrischen Verlusten unter Berücksichtigung des im Shunt und in der Wicklung auftretenden Anteils $P_{S}$ berechnet:

$$
P=\frac{N_{1}}{N_{2}} f \int_{0}^{1 / f} U(t) \cdot I(t) d t-P_{S}
$$

Des Weiteren lassen sich die magnetischen Felder aus dem Strom- bzw. Spannungsverlauf bestimmen. Aus dem Durchflutungsgesetz folgt (3.5), aus dem Induktionsgesetz (3.6):

$$
\begin{aligned}
& H(t)=\frac{N_{1} I(t)}{l_{e}}, \\
& B(t)=\frac{1}{N_{2} A_{e}} \int_{0}^{t} U\left(t^{\prime}\right) d t^{\prime}
\end{aligned}
$$

Hierbei sind $A_{e}$ der mittlere magnetische Querschnitt, $l_{e}$ die mittlere magnetische Weglänge und $V_{e}=A_{e} \cdot l_{e}$ das magnetisch wirksame Volumen nach DIN EN 60205 [86]. Aus $H(t)$ und $B(t)$ lassen sich die Hysterese und damit auch die Sättigungsmagnetisierung, die Remanenz und die Koerzitivfeldstärke bestimmen.

Einige wichtige Gerätedaten sind in Tabelle 3.5 aufgeführt [87].

\begin{tabular}{l|l}
\hline Messfrequenz: & $50 \mathrm{~Hz}-1 \mathrm{MHz}$ \\
\hline A/D Abtastrate: & 512 x Messfrequenz \\
\hline A/D Auflösung: & $12 \mathrm{bit}$ \\
\hline Messwerte: & $\hat{B}, \hat{H}, B_{r,} H_{c, \text { Verlustleistung } P}$ \\
\hline Genauigkeit: & $\begin{array}{l}\mathrm{H}-\text { Feld, B-Feld } \pm 2 \% \\
\text { Verlustleistung } P \quad \pm 5 \%\end{array}$ \\
\hline externer Verstärker: & $N F-H S A 4101$ \\
\hline
\end{tabular}

Tabelle 3.5 Charakteristik des für die Verlustmessung verwendeten Messsystems.

\subsection{Mikrostrukturuntersuchungen}

\subsubsection{Licht- und Rasterelektronenmikroskopie}

\section{Probenpräparation}

Für die Lichtmikroskopie und teilweise auch für die Rasterelektronenmikroskopie werden die Proben mittels einer metallografischen Schleif- und Poliereinrichtung der Fa. Struers 
(Modell Rotopol 22) präpariert (Anschliffpräparation). Die Proben werden kalt in Acrylharz eingebettet und dann über mehrere, feiner werdende Schleif- und Polierstufen bearbeitet, bis sich eine metallisch spiegelnde Oberfläche einstellt. Korngrenzen sind im polierten Zustand nicht erkennbar, Poren hingegen heben sich kontrastreich von der reflektierenden Oberfläche ab. Die Porosität $\Theta$ lässt sich bei genügendem Kontrast mittels eines Bildbearbeitungsprogramms einfach aus dem Flächenanteil der Poren berechnen: $\Theta=A_{\text {pore }} / A_{\text {gesamt }}\left(A_{\text {pore }}=\right.$ Porenanteil der untersuchten Fläche $\left.A_{\text {gesamt }}\right)$. Nach Exner [88] ist der Flächenanteil der Porosität ist mit dem Volumenanteil identisch:

$$
\Theta=A_{\text {pore }} / A_{\text {gesamt }}=V_{\text {pore }} / V_{\text {gesamt }} .
$$

Um die Korngrenzen sichtbar $\mathrm{zu}$ machen werden die Proben mit einer verdünnten Mischung aus Flusssäure und Salpetersäure im Verhältnis 3:1 für kurze Zeit angeätzt. In der Nähe der Korngrenzen wird hierbei verstärkt Material abgetragen, so dass sich die Korngrenzen nach dem Ätzen als Vertiefungen in der Probenoberfläche abzeichnen.

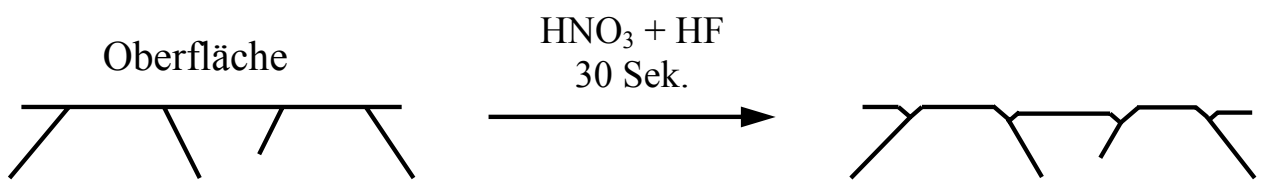

Abbildung 3.7 Materialabtrag in der Nähe der Korngrenze beim Anätzen der polierten Anschliffe.

\section{Gefügeuntersuchungen}

Die Korngröße wurde durchgängig anhand der Anzahl $N$ von einer Linie der Länge $L_{0}$ geschnittenen Körner bestimmt. Es gilt folgende einfache Beziehung für die aus einer Linearanalyse bestimmte mittlere Korngröße (Heynsche Korngröße) $\bar{L}=L_{o} / N$ [88]:

$$
\bar{L}=4 \frac{V / V_{0}}{S / V_{0}}
$$

Hierbei ist $V_{0}$ das untersuchte Testvolumen, $V$ das darin enthaltene Volumen einer untersuchten Phase und $S$ die in $V_{0}$ vorhandene Grenzfläche. Das in Abbildung 2.9 gezeigte, aus Polyedern gebildete Gefügemodell geht auf Coble zurück (Tetrakaidekaedermodell). Die Grenzflächen bestehen aus Quadraten und Hexagonen mit konstanter Kantenlänge. Ist $\bar{X}$ der Abstand zwischen den Mittelpunkten zweier benachbarter Körner, so ergibt sich aus der spezifischen Oberfläche des Polyeders:

$$
\bar{X}=\left(\frac{9}{8}+\frac{\sqrt{3}}{4}\right) \bar{L}=1,558 \bar{L}
$$




\subsubsection{Transmissionselektronenmikroskopie (TEM)}

Für die Transmissionselektronenmikroskopie werden zylinderförmige Ferritstäbe gepresst und gesintert. Aus den Zylindern mit Durchmesser $3 \mathrm{~mm}$ schneidet man mittels einer Diamanttrennscheibe runde Plättchen mit einer Höhe von $1 \mathrm{~mm}$, die mittels eines „Disc-Grinders“ planparallel geschliffen und beidseitig mit einem Muldenschleifer (Dimpler) bis auf eine Stegstärke von $20 \mu \mathrm{m}$ ausgedünnt werden. Eine weitere Dünnung erfolgt durch den Beschuss mit schräg einfallenden Ar - Ionenstrahlen (Ionenätzen), bis zur Ausbildung eines Durchbruchs in der Mitte der Probe. In Abbildung 3.8 und in Tabelle 3.6 sind die einzelnen Präparationsschritte schematisch dargestellt.

a)

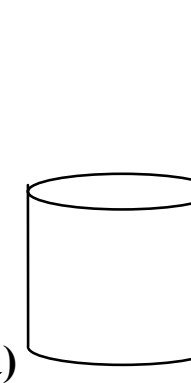

Abbildung 3.8

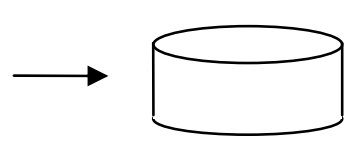

b)

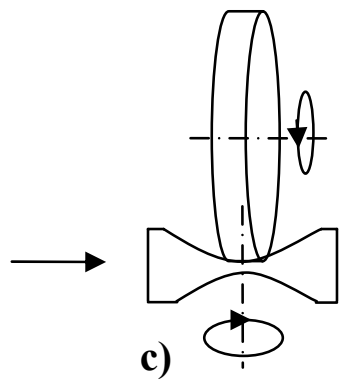

c)

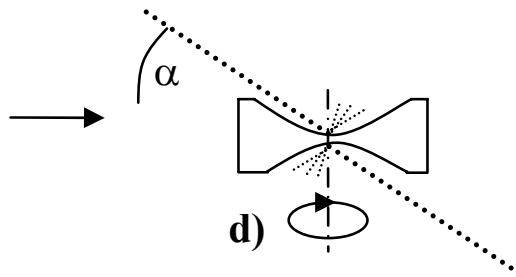

\begin{tabular}{c|l|l}
\hline $\begin{array}{c}\text { Teil- } \\
\text { abbildung }\end{array}$ & Prozessschritt & Prozessparameter \\
\hline a) & Sintern eines Stabes mit $\varnothing 3 \mathrm{~mm}$ & $1380^{\circ} \mathrm{C}, 12 \mathrm{~h}$, Standardbedingungen \\
\cline { 2 - 3 } & $\begin{array}{l}\text { Abtrennen von Scheiben der } \\
\text { Höhe } \approx 1 \mathrm{~mm}\end{array}$ & \\
\hline b) & $\begin{array}{l}\text { Schleifen von planparallelen } \\
\text { Scheiben, Höhe }=200 \mu \mathrm{m}\end{array}$ & Nassschleifpapier mit Körnung 1200 \\
\hline c) & $\begin{array}{l}\text { beidseitiges Einschleifen von } \\
\text { Mulden mit einem Dimpler der } \\
\text { Fa. Gatan }\end{array}$ & $\begin{array}{l}\text { Schleifmittel: } 1 / 4 \mu \mathrm{m} \text { Diamant- } \\
\text { suspension DP-Spray (Struers) }\end{array}$ \\
\hline d) & $\begin{array}{l}\text { beidseitiges Ionenätzen mit Ar- } \\
\text { Ionen }\end{array}$ & $\begin{array}{l}\text { Einfallswinkel: } \alpha=10-15^{\circ} \\
\text { Beschleunigunggspannung: } 5 \mathrm{kV} \\
\text { Probenstrom: ca. } 5 \mu \mathrm{A}\end{array}$ \\
\hline
\end{tabular}

Tabelle 3.6 $\quad$ Prozessparameter für die Herstellung von TEM-Proben, Erläuterungen zu Abbildung 3.8.

Probleme bereitet die Sprödigkeit der Proben. Insbesondere bei einem $\mathrm{Bi}_{2} \mathrm{O}_{3}$-Anteil $>$ 0,4 Gew.\% kommt es häufig zum Ausbruch größerer Bereiche während der Probenpräparation oder beim Einschleusen der Proben in das Elektronenmikroskop.

Die TEM-Untersuchungen wurden größtenteils mit einem Elektronenmikroskop Philips EM 420 ST an der Universität Göttingen durchgeführt. Das Gerät hat eine Beschleunigungsspannung von maximal $120 \mathrm{kV}$ und ein maximales Auflösungsvermögen von 
$0,3 \mathrm{~nm}$ und ermöglicht neben der Bild- und Beugungsanalyse zusätzlich die ortsaufgelöste Messung der Elementverteilung über die spektrometrische Auswertung der entstehenden Röntgenstrahlung (EDX - Analyse).

Die TEM-Untersuchungen an den mit Mikrowelle gesinterten Proben entstanden am MPI für Mikrostrukturanalyse in Halle mit den Mikroskopen Typ Philips CM 20 T und Philips CM 20 FEG mit maximalen Beschleunigungsspannungen von $200 \mathrm{kV}$. 


\section{Messergebnisse}

\subsection{Einfluss schmelzfließender Phasen auf die Eigenschaften von MnZn-Ferriten}

\subsubsection{Untersuchungen zur Wismutoxid-Ferrit-Phasengrenze an Presslingen}

Um einen Einblick in die grundlegende Wechselwirkung einer Wismutoxidschmelze mit einem Ferritpressling zu bekommen, wurden zweiphasige Grünkörper (Tabletten) hergestellt. Hierzu schichtet man Ferritpresspulver ${ }^{[i]}$ (vgl. 3.2.1) und granuliertes Wismutoxid ähnlicher Sekundärkorngröße in einer Pressform übereinander und verpresst die beiden Pulver uniaxial. Danach erfolgt ein einstündiges Auslagern der Proben bei jeweils $600{ }^{\circ} \mathrm{C}, 700{ }^{\circ} \mathrm{C}, 800{ }^{\circ} \mathrm{C}$ und $900{ }^{\circ} \mathrm{C}$. Die Entwicklung der Grenzfläche ist in den Abbildung 4.1 a) - d) in lichtmikroskopischen Aufnahmen dargestellt.
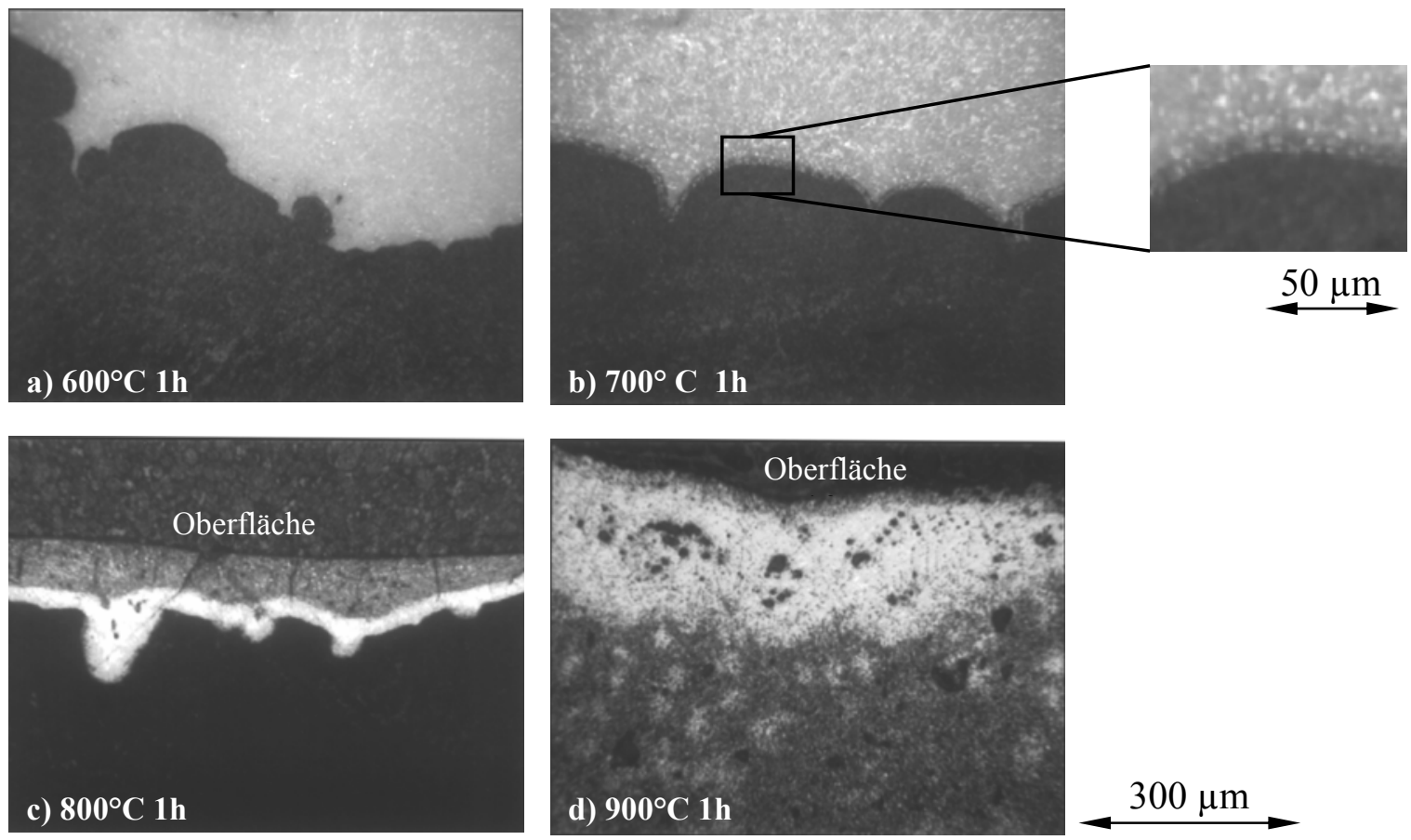

Abbildung 4.1 Lichtmikroskopische Aufnahmen zur Entwicklung der Wismutoxid-FerritPhasengrenze. Teilbild a) zeigt den Verlauf nach dem Pressen, b) das Einsetzen starker $\mathrm{Bi}_{2} \mathrm{O}_{3}$-Diffusion bei Temperaturen von $700{ }^{\circ} \mathrm{C}$, c) das Aufschmelzen des Wismutoxids, wobei sich zwei übereinander liegende Schichten bilden und d) das Eindringen der Schmelze in den offenporigen Ferritpressling.

Nach der $600{ }^{\circ} \mathrm{C}$-Behandlung (Teilbild a) ist kein Unterschied zur unbehandelten Probe erkennbar. Die Grenzfläche zwischen den beiden Pulverschichten ist scharf, ihr Verlauf

[i] Genau genommen handelt es sich um eine kalzinierte und mit organischen Binde- und Gleitmitteln versehene Mischung der Metalloxide mit einem Anteil an magnetischer Spinellphase von ca. 5\%. 
verdeutlicht das unterschiedliche Verhalten der beiden Granulate beim Pressen der Tabletten. Das verhältnismäßig weiche $\mathrm{Bi}_{2} \mathrm{O}_{3}$-Granulat wird bei der Pressung deformiert und ,umfließt“ die härteren Ferritkörner. Stellenweise bilden sich kleine „Wismutoxidkeile“ zwischen den Ferritkörnern aus.

Bei auf $700{ }^{\circ} \mathrm{C}$ erhöhter Auslagerungstemperatur setzt starke Diffusion ein. Dies zeigt sich an einem deutlichen Kristallwachstum in der Wismutoxidschicht und am Entstehen einer ca. $10 \mu \mathrm{m}$ breiten Interdiffusionsschicht an der Grenzfläche (Ausschnitt von Abbildung 4.1 b)).

Die Wärmebehandlung bei $800{ }^{\circ} \mathrm{C}$ bewirkt die Bildung von wismutoxidreicher Schmelze. Die Schmelztemperatur des Wismutoxids $\left(825^{\circ} \mathrm{C}\right.$, vgl. Abbildung 2.12) wird zwar nicht erreicht, die eutektische Temperatur der entstehenden Mischphasen liegt jedoch erwartungsgemäß zwischen $750{ }^{\circ} \mathrm{C}$ und $800{ }^{\circ} \mathrm{C}$.

Nach dem Erstarren der Schmelze findet sich der in Abbildung 4.1 c) und Abbildung 4.2 gezeigte dreischichtige Aufbau.
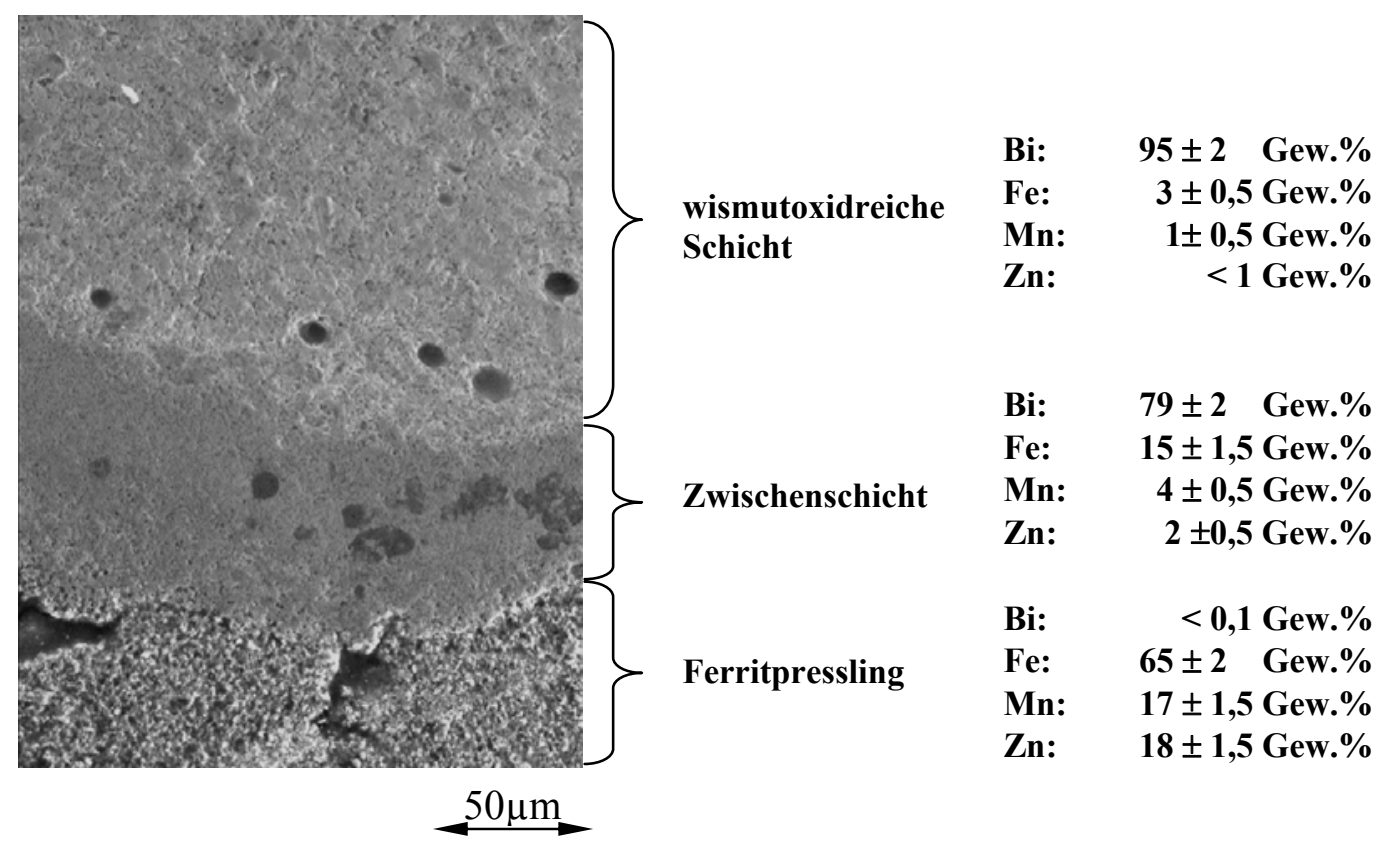

Abbildung 4.2 REM-Aufnahme der bei $800^{\circ} \mathrm{C}$ ausgelagerten Probe. Die angegebenen Konzentrationen sind über eine größere Fläche gemittelt.

In der obersten, wismutoxidreichen Schicht lassen sich nur geringe Mengen der anderen Oxide nachweisen. Im Rückstreubild des Elektronenmikroskops wird sichtbar, dass es sich im Wesentlichen um Ausscheidungen in einer Matrix aus beinahe reinem Wismutoxid handelt (vgl. Abbildung 4.4). Die darunterliegende Zwischenschicht entspricht in ihrer Zusammensetzung ungefähr diesen Ausscheidungen. Die in der Zwischenschicht erkennbaren dunkleren Bereiche zeigen bzgl. der Elementverteilung 
keine signifikanten Abweichungen zur Umgebung. Die unterste Schicht lässt das offenporige und defektbehaftete Gefüge des Ferritpresslings erkennen. Ihre Zusammensetzung ist homogen, der Gehalt an Wismutoxid liegt unter der Nachweisgrenze.

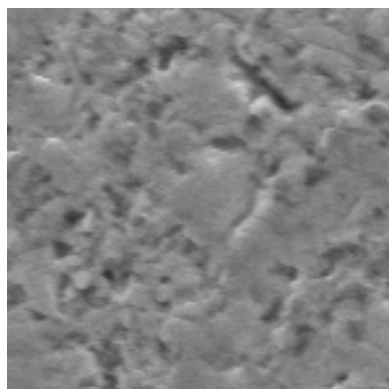

a) $\mathrm{Bi}_{2} \mathrm{O}_{3}$-reiche Schicht

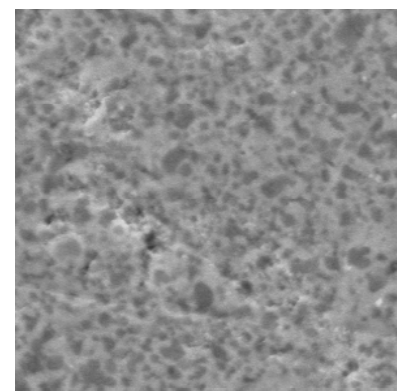

b) Zwischenschicht

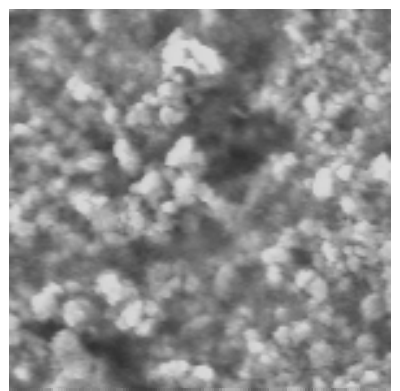

$5 \mu \mathrm{m}$

Abbildung 4.3 Detailaufnahmen der einzelnen Schichten.

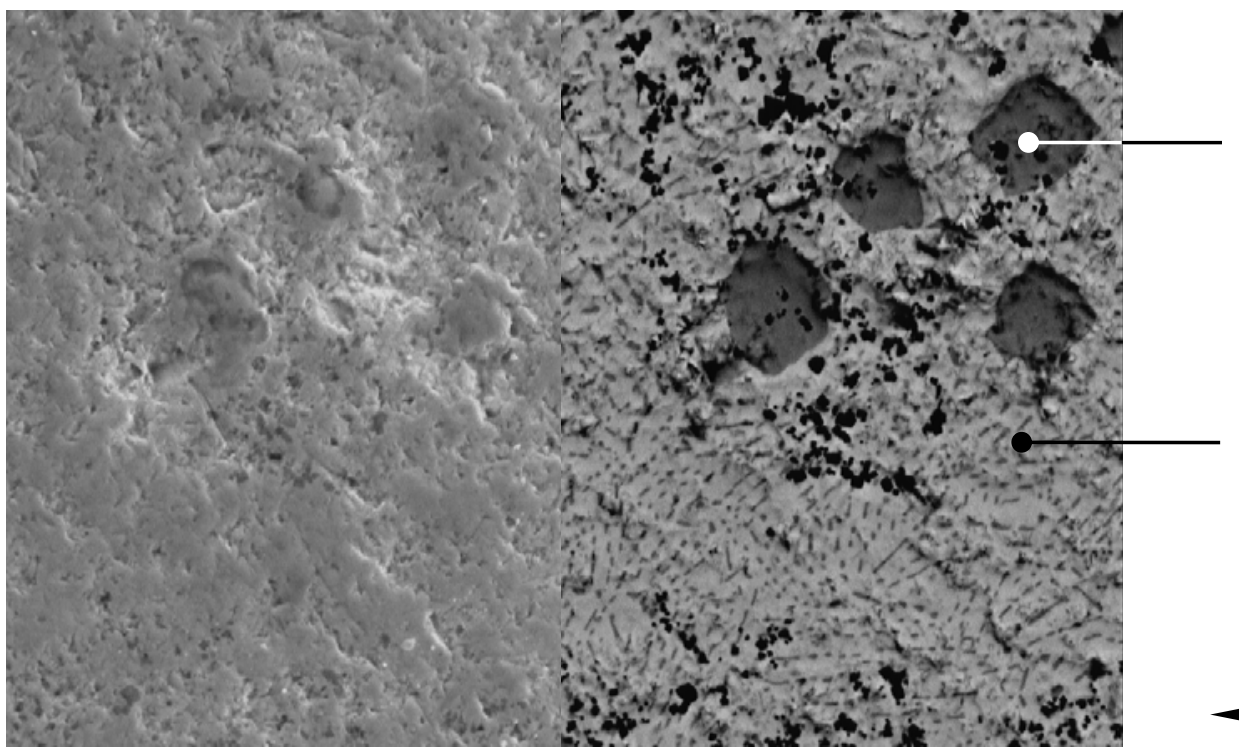

Ausscheidung:

Bi: $83 \pm 2$ Gew. $\%$

Fe: $13 \pm 1$ Gew. \%

Mn: $3 \pm 0,5$ Gew. $\%$

Zn: ca. 0,5 Gew. $\%$

Matrix:

Bi: $99 \pm 2$ Gew.\%

Fe: $\quad 1 \pm 0,5$ Gew. $\%$

Mn: ca. 0,3 Gew.\%

Zn: ca. 0,2 Gew. $\%$

Abbildung 4.4 Sekundärelektronen- (links) und Rückstreuelektronenaufnahme (rechts) der oberen, wismutreichen Deckschicht. Die Aufnahme im Rückstreumodus zeigt Ausscheidungen, in denen insbesondere der Eisengehalt deutlich erhöht ist.

Die letzte in Abbildung 4.1 gezeigte, bei $900{ }^{\circ} \mathrm{C}$ für eine Stunde behandelte Probe zeigt keine scharfen Phasengrenzen zwischen geschmolzener Wismutoxidschicht und Ferrit. Ein Teil der Schmelze befindet sich noch an der Oberfläche, Teile infiltrieren den offenporigen, nur leicht versinterten Ferrit und reichern sich in schlecht verdichteten Bereichen bzw. in Hohlräumen an. Der Formkörper verliert hierdurch an mechanischer Stabilität und wird leicht deformiert.

\subsubsection{Mikrostruktur bei Ferriten mit Wismutoxiddotierung}

Anders als bei den untersuchten Grenzflächen in Presslingen zeigt sich die Wirkung des Schmelzflusses, wenn $\mathrm{Bi}_{2} \mathrm{O}_{3}$ nur als Dotierung in Mengen von einigen hundert ppm in 
das Ferritgranulat homogen eingebracht wird und die thermische Behandlung bei einer für hochpermeable MnZn-Ferrite typischen Sintertemperatur von $1380{ }^{\circ} \mathrm{C}$ stattfindet.

Im Folgenden Abschnitt wird zuerst der Einfluss einer Wismutoxiddotierung im Bereich von $0-800 \mathrm{ppm}$ auf das Gefüge untersucht. Hierbei werden alle Proben unter Standardbedingungen von $12 \mathrm{~h}$ bei $1380{ }^{\circ} \mathrm{C}$ gesintert. Danach erfolgt eine genauere Betrachtung des Kornwachstumsprozesses. Dafür wurde in einer Versuchsreihe die Sinterdauer variiert. Die verwendeten Probenbezeichnungen und Zusammensetzungen folgen der Aufstellung in Tabelle 3.1.

\section{$\underline{\text { Isotherme Sinterzeiten von } 12 \mathrm{~h}}$}

Die durchgeführten Mikrostrukturuntersuchungen belegen den starken Einfluss, den die Anwesenheit geringster Mengen einer schmelzfließenden Phase auf die Gefügebildung hat. Die Ergebnisse der Untersuchungen sind in Tabelle 4.1 zusammengefasst. [89, 90]

\begin{tabular}{c|c|c|c|l}
\hline $\begin{array}{c}\text { Probenbe- } \\
\text { zeichnung }\end{array}$ & $\begin{array}{c}\text { Gehalt an } \\
\mathrm{Bi}_{2} \mathrm{O}_{3} \\
{[\mathrm{Gew.} \mathrm{\% ]}}\end{array}$ & $\begin{array}{c}\text { Porosität } \\
\Theta \\
{[\%]}\end{array}$ & $\begin{array}{c}\text { Korngröße } \\
\bar{X} \\
{[\mu \mathrm{m}]}\end{array}$ & Charakterisierung des Gefüges \\
\hline HP1 & 0,00 & 2,7 & 19,9 & gleichmäßig, leicht bimodal \\
\hline HP1_Bi2 & 0,02 & 1,7 & 28,4 & gleichmäßig, leicht bimodal \\
\hline HP1_Bi3 & 0,03 & 1,4 & 25,8 & gleichmäßig, leicht bimodal \\
\hline HP1_Bi4 & 0,04 & 2,4 & $\begin{array}{c}>1000 \\
\text { bzw. 29,7 }\end{array}$ & $\begin{array}{l}\text { Duplexstruktur, im Innern der Probe } \\
\text { Riesenkornwachstum (X }>1 \text { mm), in } \\
\text { einer 500 - 700 } \mu \text { breiten Randzone } \\
\text { findet normales Kornwachstum statt. }\end{array}$ \\
\hline HP1_Bi6 & 0,06 & 3,8 & $>1000$ & $\begin{array}{l}\text { Duplexstruktur wie bei HP1_Bi4, } \\
\text { normales Kornwachstum findet nur } \\
\text { bzw. 19,2 } \\
\text { noch in einer ca. 50 } \mu \mathrm{m} \text { breiten }\end{array}$ \\
\hline HP1_Bi8 & 0,08 & 3,5 & $>1000$ & $\begin{array}{l}\text { Randzone statt. } \\
\text { Das Gefüge wird vollständig aus } \\
\text { wenigen, sehr großen Körnern } \\
\text { gebildet. }\end{array}$ \\
\hline
\end{tabular}

Tabelle 4.1 Ü̈bersicht über die Änderung der Gefügeeigenschaften bei Wismutoxidzugabe. Die isotherme Haltezeit beträgt bei allen untersuchten Gefügen 12h, die Sintertemperatur $1380{ }^{\circ} \mathrm{C}$.

Schon bei den mit 200 ppm und 300 ppm dotierten Proben HP1_Bi2 und HP1_Bi3 tritt eine Vergrößerung des mittleren Korndurchmessers von $20 \mu \mathrm{m}$ auf ca. 25 - $30 \mu \mathrm{m}$ auf 
(Abbildung 4.5 a) und b). Das Gefüge ist wie bei der undotierten Probe leicht bimodal, ansonsten aber weitestgehend homogen.

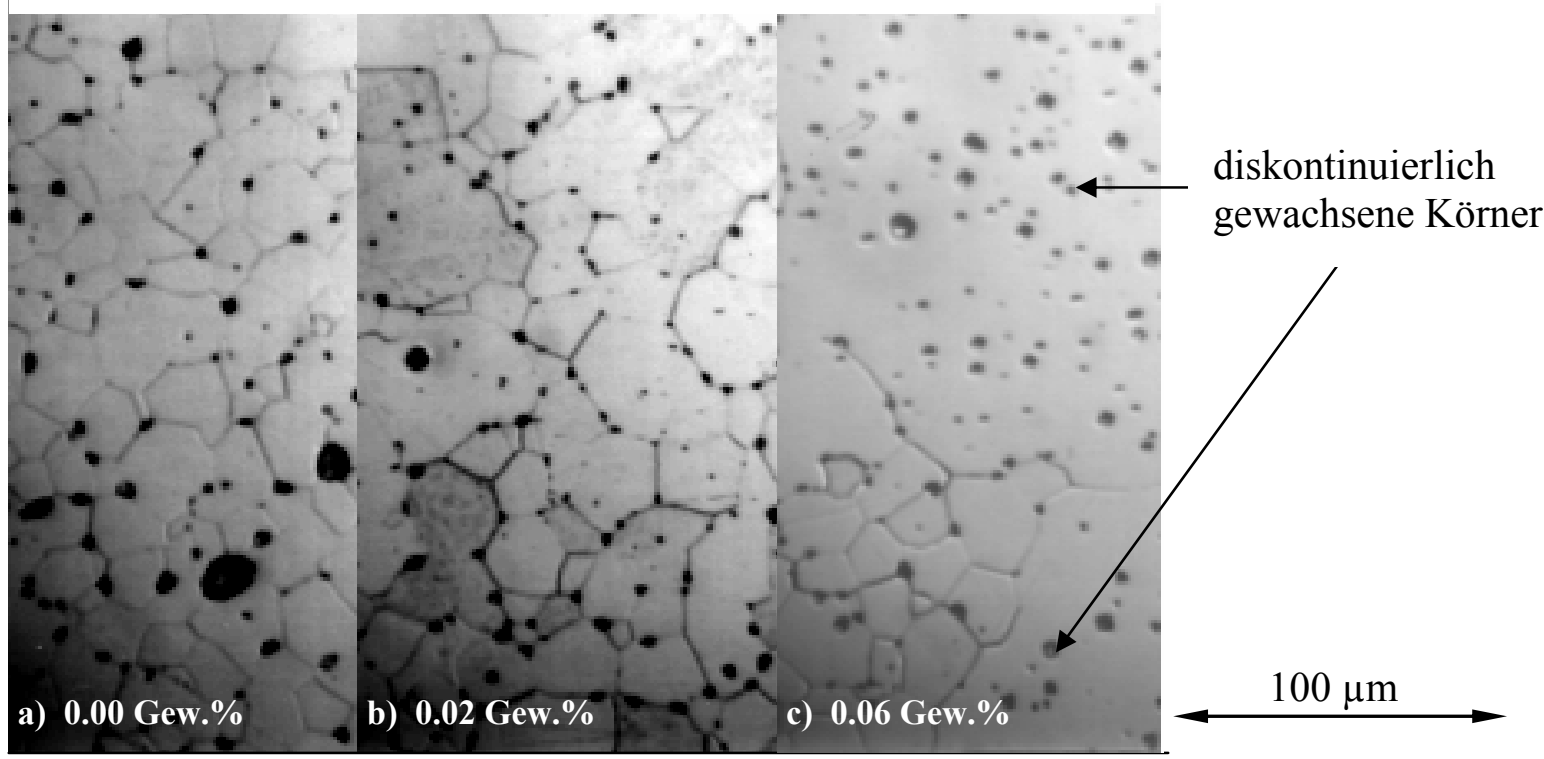

Abbildung 4.5 Änderung des Gefüges bei der Zugabe von $\mathrm{Bi}_{2} \mathrm{O}_{3}$ und die Entstehung von diskontinuierlichem Kornwachstum.

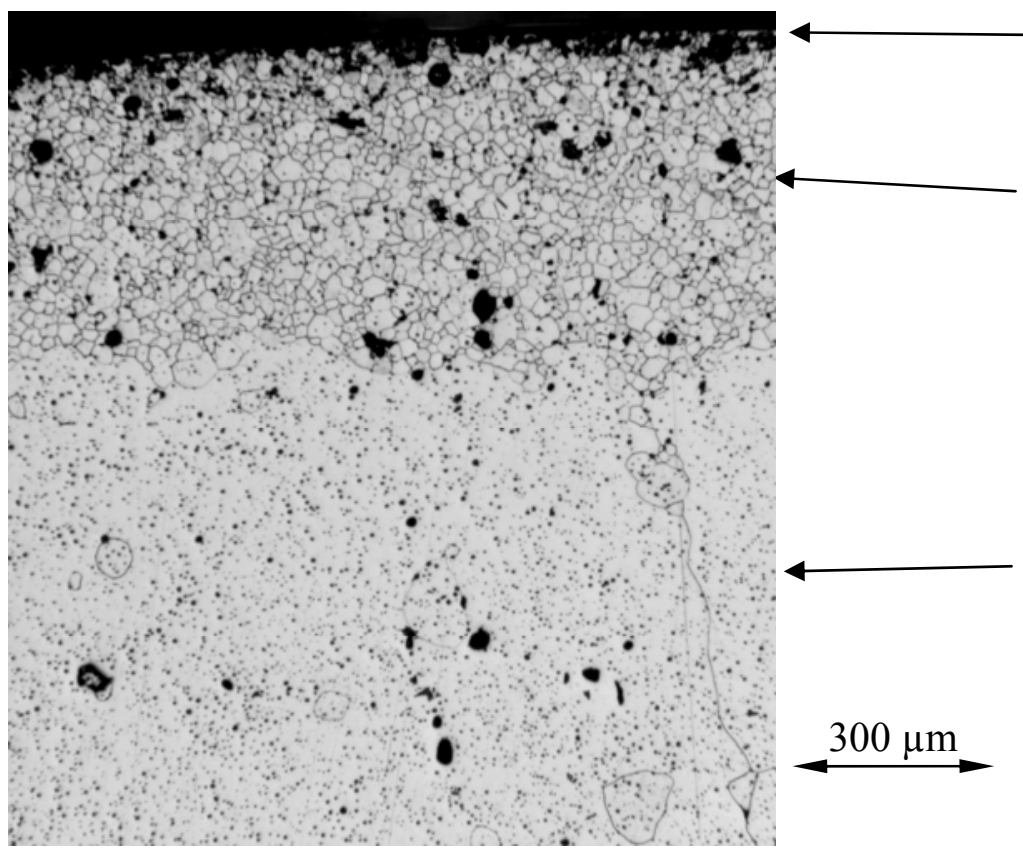

Oberfläche

Schicht mit normalem

Kornwachstum

Abbildung 4.6

Kornstruktur der mit 0,04 Gew.\% $\mathrm{Bi}_{2} \mathrm{O}_{3}$ dotierten Probe HP_Bi4. In einer 500 $700 \mu \mathrm{m}$ breiten Randzone findet sich normalgewachsenes Gefüge, während im Probeninneren diskontinuierlich gewachsene Körner mit hoher intragranularer Porosität erkennbar sind.

Dies ändert sich ab 0,04 Gew.\% Wismutoxidgehalt (Proben HP_Bi4 und HP_Bi6).

Im Inneren des gesinterten Ferritmaterials entstehen diskontinuierlich wachsende Körner mit hochmobilen Korngrenzen, die sich im Verlauf der isothermen Sinterphase auf Kosten des normalgewachsenen Gefüges ausdehnen. Nach der Temperaturbehandlung 
besteht das Gefüge im Probeninneren aus wenigen, sehr großen Körnern mit hoher intragranularer Porosität. Am Probenrand verbleibt eine Zone mit normalgewachsenem Gefüge (Abbildung 4.6). Der Gehalt an Wismutoxid beeinflusst nur noch die Breite dieser Zone. Sie verringert sich von ca. $600 \mu \mathrm{m}$ bei Probe HP1_Bi4 auf ca. $50 \mu \mathrm{m}$ bei Probe HP1_Bi6. Bei Probe HP1_Bi8 ist kein kontinuierlich gewachsenes Gefüge mehr vorhanden.

\section{$\underline{\text { Untersuchungen an intergranularen Poren }}$}

Untersucht man an planen, geschliffenen und geätzten Flächen (vgl. Abschnitt 3.5.1) der Probe HP1_Bi4 die Form der intergranularen Poren, so findet man für Korngrenzen zwischen normal- und diskontinuierlich gewachsenen Körnern Konfigurationen wie in Abbildung 4.7. In Übereinstimmung mit der in Abschnitt 2.2.2 dargestellten Theorie bewegt sich die Korngrenze in das normalgewachsene Gefüge und wird dabei von intergranularen, sich mitbewegenden Poren gehalten. Analog zu Abbildung 2.11 lässt sich der mittlere Öffnungswinkel $\bar{\psi}_{d n}{ }^{[\mathrm{i}]}$ und der Haltewinkel $\theta$ bestimmen. Der Vergleich der Öffnungswinkel $\bar{\psi}$ lässt Aussagen über das Verhältnis von Korngrenzen- und Oberflächenenergie im Poreninneren zu (s. Gleichung 2.30).
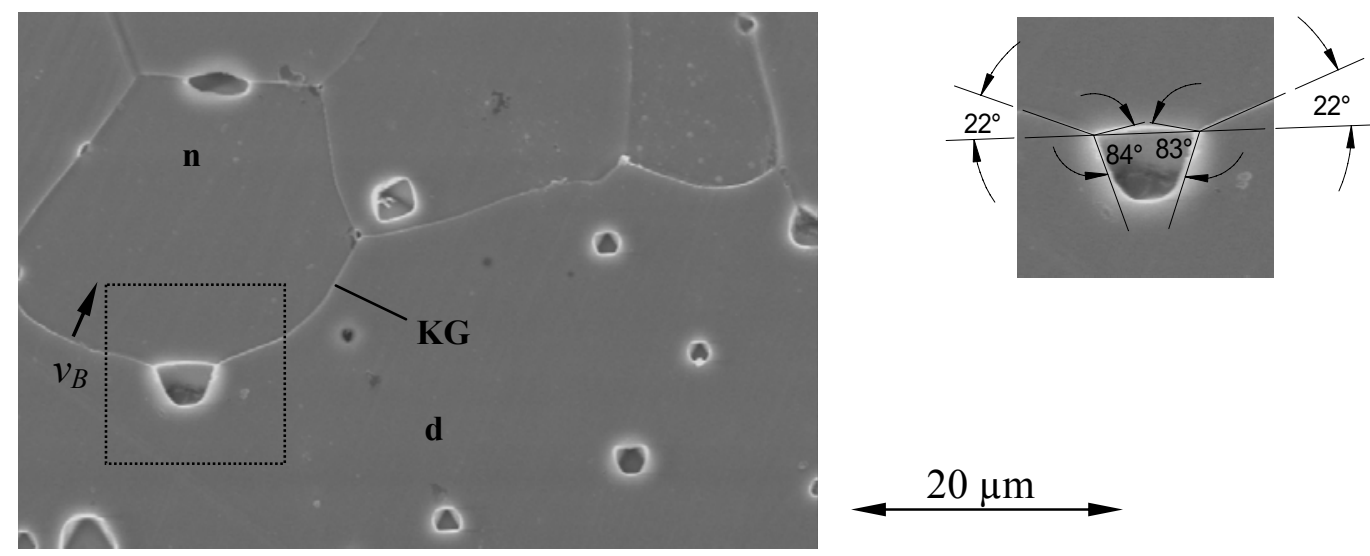

Abbildung 4.7 Übergangsbereich zwischen normal (n) und diskontinuierlich gewachsenem Gefüge (d) bei Probe HP_Bi4. Die Korngrenze (KG) bewegt sich mit der Korngrenzengeschwindigkeit $\bar{v}_{B}$ in das normalgewachsene Korn. Die Pore mit Öffnungswinkel $\psi_{d n} \approx 84^{\circ}$ und Haltewinkel $\theta \approx 22^{\circ}$ bewegt sich mit der Korngrenze mit.

Abbildung 4.7 zeigt jedoch auch anhand der intragranularen Poren im diskontinuierlich gewachsenen Korn, dass sich die Porenform beim Herauspräparieren der Korngrenzen

[i] Die Mittelwertbildung erfolgt über die beiden, an einer Pore gemessenen Öffnungswinkel. Der Index $d n$ steht für ein Korngrenze zwischen diskontinuierlich- und normalgewachsenem Gefüge, der Index $n$ für Korngrenzen innerhalb des normalgewachsenen Gefüges. 
durch Ätzen häufig verändert. Es ist deshalb möglich, dass der mittlere Öffnungswinkel $\bar{\psi}_{d n}$ mit $84^{\circ}$ fälschlicherweise als zu klein angenommen wird. Dies legt auch die Untersuchung an intergranularen Poren im normalgewachsenen Gefüge nahe (s. Tabelle 4.2). Während an den geätzten Schliffflächen relativ große Streubreiten und vergleichsweise niedrige Öffnungswinkel auftreten, so sind die an den Bruchflächen gemessenen mittleren Öffnungswinkel eng verteilt und verhältnismäßig groß.

\begin{tabular}{l|c|c}
\hline $\begin{array}{l}\text { stabile Korngrenzen im } \\
\text { normalgewachsenen Gefüge }\end{array}$ & geätzte Schlifffläche & Bruchfläche \\
\hline mittlerer Öffnungswinkel $\bar{\psi}_{n}$ & $75^{\circ}-95^{\circ}$ & $100^{\circ}-105^{\circ}$ \\
\hline
\end{tabular}

Tabelle 4.2 Öffnungswinkel intergranularer Poren im normalgewachsenen Gefüge.

Allerdings lässt die Form der Bruchflächen vermuten, dass das Gefüge häufig intergranular, also entlang der Korngrenzflächen bricht. Folglich liegt die in Abbildung 4.8 a) gezeigte Pore möglicherweise in einem Tripelpunkt und die Pore in Teilabbildung b) wurde wahrscheinlich ursprünglich von vier Körnern umgrenzt.
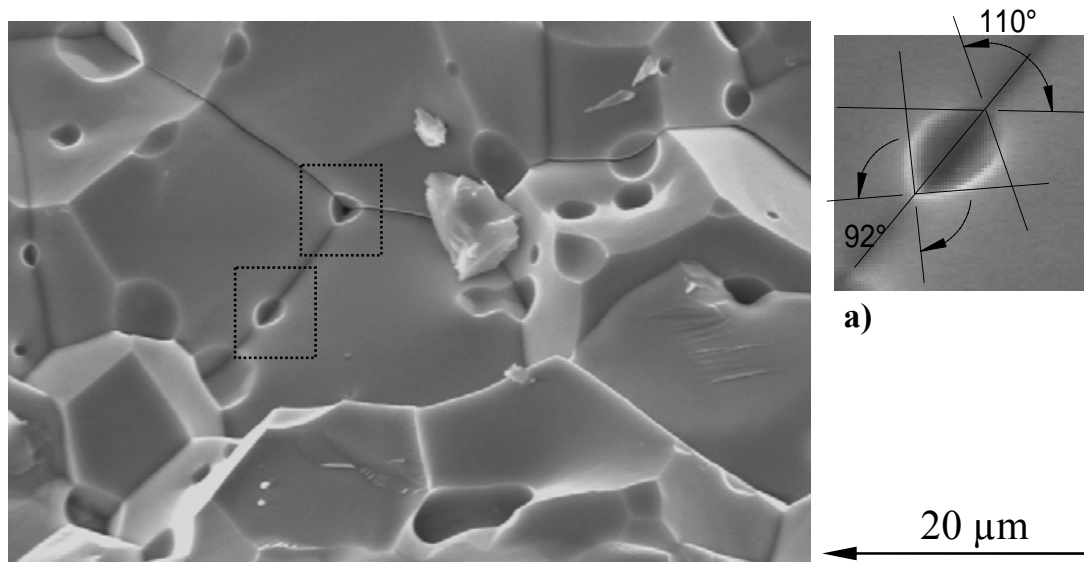

a) $20 \mu \mathrm{m}$

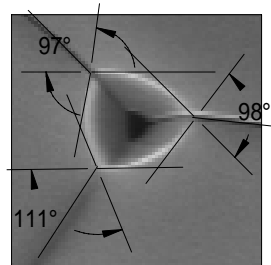

b)

Abbildung 4.8 Analyse des Öffnungswinkels $\psi_{n}$ an einer intergranularen Pore und einem Tripelpunkt.. Untersucht wurde die Bruchflächen des normalgewachsenen Gefüges der Probe HP_Bi4.

\section{Einfluss der Porosität $\Theta$}

Wird für die einzelnen Proben der Flächenanteil der Porosität $\Theta$ untersucht, so findet sich der in Abbildung 4.9 dargestellte Zusammenhang zwischen Porosität, Korngröße, Wismutoxidgehalt und vorhandener Gefügestruktur. Bei der undotierten Probe mit überwiegend intergranularem Porenanteil liegt die Porosität bei ca. 2,7\%. Bei den niedrig dotierten Proben HP1_Bi2 und HP1_Bi3 nimmt der intragranulare Porenanteil etwas zu, die Gesamtporosität aber auf 1,5 \% ab. Einerseits erhöhen schon Wismutoxidanteile von 200 ppm die Korngrenzenbeweglichkeit zumindest lokal so stark, dass es vereinzelt zu einem Loslösen von Korngrenzen aus ihrer Verankerung und zur Entstehung von 
intrakristallinen Poren kommt. Andererseits können sich die intergranularen Poren aufgrund erhöhter Korngrenzendiffusion auch besser abbauen (vgl. Abbildung 4.5 und Abbildung 4.10).

Bei den höher dotierten Proben nimmt die Porosität wieder zu und wächst auf über 3,5 \% bei Dotierungen $>600 \mathrm{ppm}$ an. Es handelt sich hierbei zum überwiegenden Teil um intragranulare Porosität in diskontinuierlich gewachsenen Körnern. Die Korngrenzengeschwindigkeit ist nun so hoch, dass Poren für schnell wachsende Körner kein Hindernis mehr darstellen. Der Vorteil einer verbesserten Diffusion in der Korngrenze bewirkt keine Verringerung der Porosität mehr.

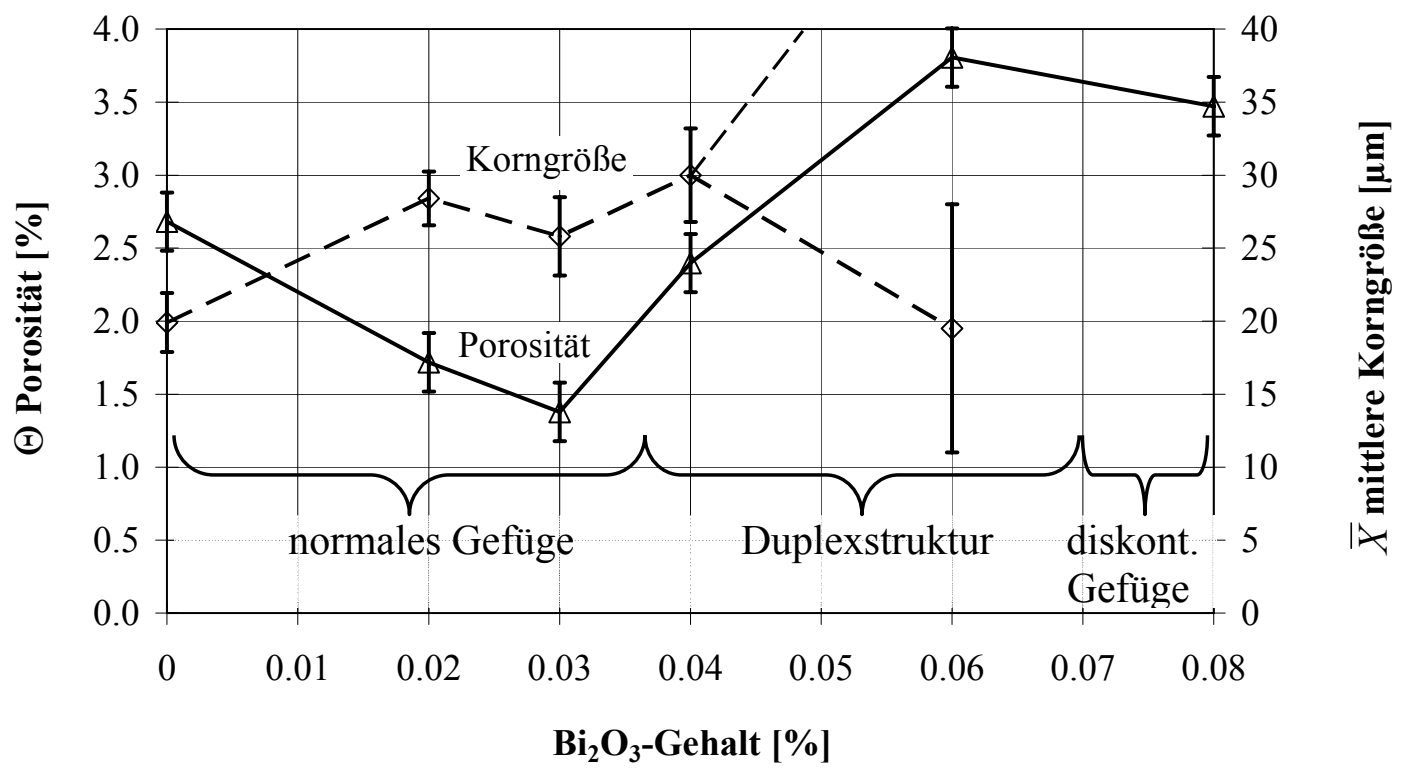

Abbildung 4.9 Änderung von Porosität und Korngröße in Abhängigkeit vom $\mathrm{Bi}_{2} \mathrm{O}_{3}$-Gehalt. Bei der Korngröße sind nur die kontinuierlich gewachsenen Körner erfasst.

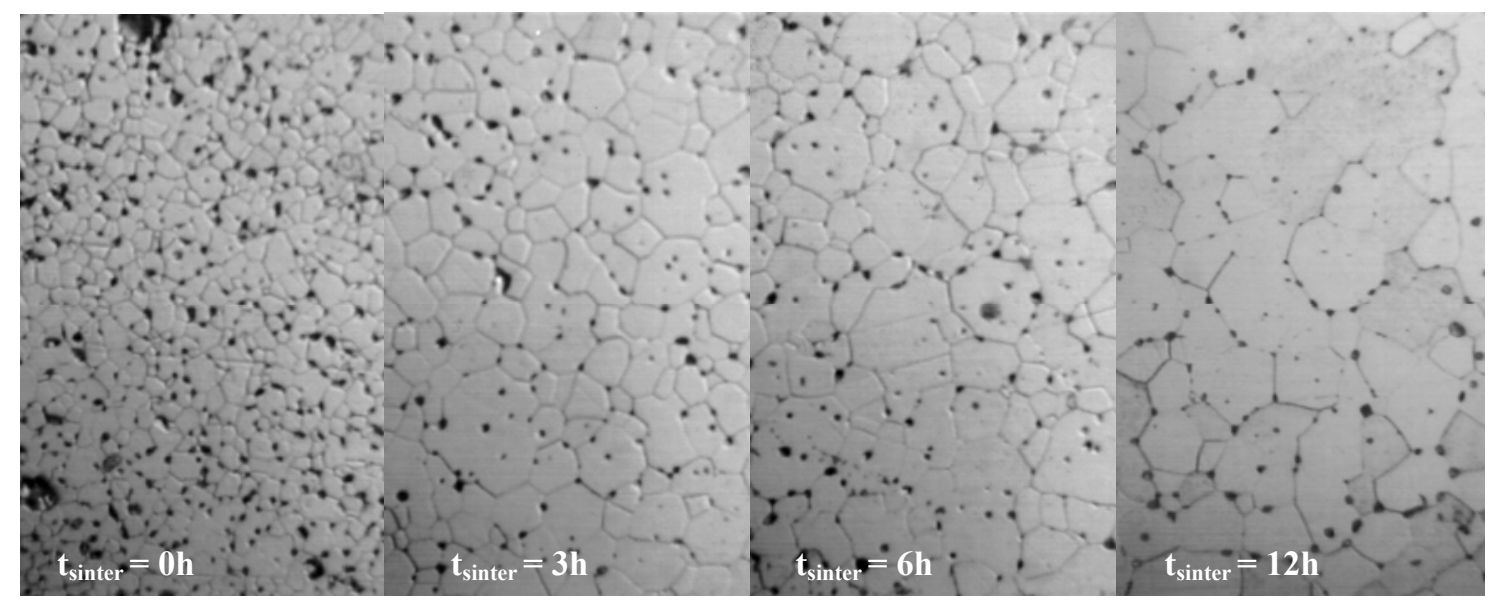

Abbildung 4.10 Veränderung von Porosität und Korngröße in Abhängigkeit von der isothermen Sinterzeit für die

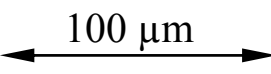
mit 0,02 Gew.\% dotierte Probe. 


\section{Kornwachstum und Änderung der Aktivierungsenergie}

Das Kornwachstum bei konstanter Sintertemperatur wird durch Gleichung 2.23 beschrieben. Zur Bestimmung des Exponenten $n$ wurde eine Versuchsreihe mit konstanter Sintertemperatur und isothermen Haltezeiten von $0 \mathrm{~h}, 3 \mathrm{~h}, 6 \mathrm{~h}$ und $12 \mathrm{~h}$ durchgeführt und die Änderung der Korngröße bei den Proben mit normalgewachsenem Gefüge bestimmt. Die Ergebnisse der Untersuchungen sind in Tabelle 4.3 dargestellt.

\begin{tabular}{c|c|c|c|c|c|c}
\hline $\begin{array}{c}\mathrm{Bi}_{2} \mathrm{O}_{3} \text {-Gehalt } \\
{[\mathrm{Gew} \% \text { \% }}\end{array}$ & $\begin{array}{c}t_{\text {sinter }}=0 \mathrm{~h} \\
\bar{X}[\mu \mathrm{m}]\end{array}$ & $\begin{array}{c}t_{\text {sinter }}=3 \mathrm{~h} \\
\bar{X}[\mu \mathrm{m}]\end{array}$ & $\begin{array}{c}t_{\text {sinter }}=6 \mathrm{~h} \\
\bar{X}[\mu \mathrm{m}]\end{array}$ & $\begin{array}{c}t_{\text {sinter }}=12 \mathrm{~h} \\
\bar{X}[\mu \mathrm{m}]\end{array}$ & $n$ & $\begin{array}{c}\widetilde{k} \\
{\left[\mu \mathrm{m}^{3} / \mathrm{h}\right]}\end{array}$ \\
\hline 0,00 & 10,4 & 14,7 & 16,4 & 19,9 & 3,2 & 580 \\
& $\pm 0,8$ & $\pm 2,3$ & $\pm 0,6$ & $\pm 2,0$ & & \\
\hline 0,02 & 10,4 & 18,7 & 20,7 & 28,4 & 2,3 & 1740 \\
& $\pm 1,2$ & $\pm 1,6$ & $\pm 2,3$ & $\pm 1,6$ & & \\
\hline 0,03 & 11,0 & 18,8 & 20,1 & 25,8 & 3,2 & 1330 \\
& $\pm 0,8$ & $\pm 1,3$ & $\pm 1,6$ & $\pm 2,7$ & & \\
\hline
\end{tabular}

Tabelle 4.3 Einfluss der Sinterzeit auf das Kornwachstum bei den Proben mit normalgewachsenem Gefüge.

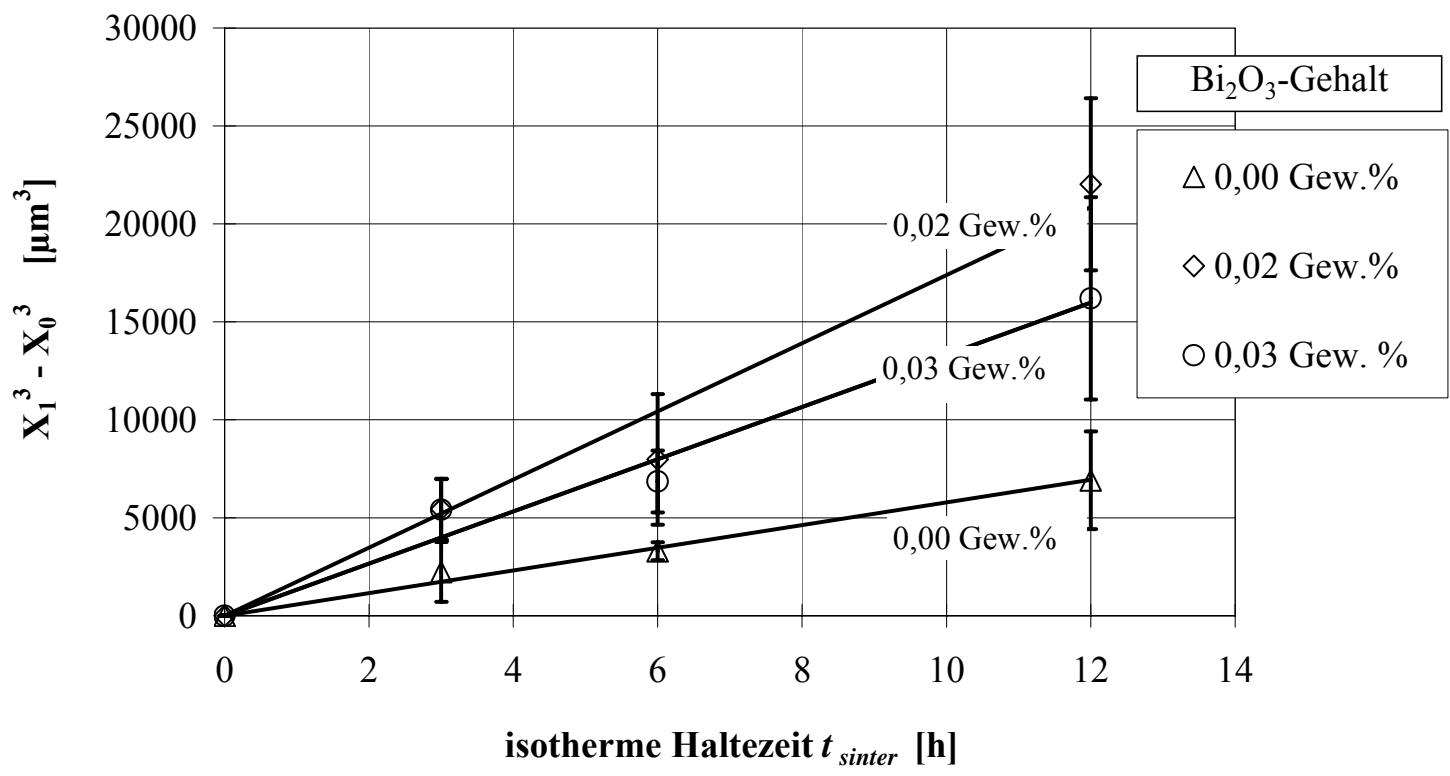

Abbildung 4.11 Zusammenhang zwischen Korndurchmesser $\bar{X}$ und Haltezeit $t_{\text {sinter für das in }}$ Gleichung 2.23 beschriebene Wachstumsgesetz mit $n=3$.

Der Exponent $n$ wird gerade so gewählt, dass der Korrelationskoeffizient der Geraden $\bar{X}^{n}-\bar{X}_{0}^{n}=\widetilde{k} t_{\text {sinter }}$ maximal ist. Übereinstimmend ergibt sich bei der wismutoxidfreien und der mit 0,03 Gew.\% dotierten Mischung $n \approx 3,2$. Bei der mit 0,02 Gew.\% dotierten Mischung findet man $n \approx 2,3$. Die bei der Messung auftretenden Streubreiten sind relativ groß, so dass eine präzise Bestimmung des Exponenten nicht möglich ist. Für die weitere 
Auswertung wurde deshalb $n=3$ gewählt (vgl. Abbildung 4.11). Dies passt gut zu den erzielten Resultaten und wird in der Literatur sowohl für Korngrenzen, deren Beweglichkeit durch Ausscheidungen, Poren oder Verunreinigungen gehemmt ist, als auch für die Anwesenheit schmelzfließender Phasen angegeben [38, 51, 72, 91].

Als wichtiges Ergebnis lässt sich aus den Geradensteigungen die Differenz der Aktivierungsenergien zwischen der undotierten Probe $\left(Q_{1}, \widetilde{k}_{1}\right)$ und den dotierten Proben $\left(Q_{2}, \widetilde{k}_{2}\right)$ abschätzen $\left(T=T_{\text {sinter }}=1653 \mathrm{~K}\right)$ :

$$
\ln \left(\frac{\widetilde{k}_{1}}{\widetilde{k}_{2}}\right)=\frac{Q_{2}-Q_{1}}{k_{B} T} .
$$

Sie beträgt $\Delta Q \approx-0,15 \mathrm{eV}$ bei Zugabe von 0,02 Gew. $\% \mathrm{Bi}_{2} \mathrm{O}_{3}$ und $\Delta Q \approx-0,11 \mathrm{eV}$ bei Zugabe von 0,03 Gew.\% $\mathrm{Bi}_{2} \mathrm{O}_{3}$. Für diskontinuierlich wachsende Körner ist wie in Abschnitt 2.2.1 erwähnt, die zugrundeliegende Gleichung 2.23 nicht gültig.

\subsubsection{Mikrostruktur bei Molybdäntrioxiddotierung}

Neben Wismutoxid wurde Molybdäntrioxid als weiteres niedrigschmelzendes Additiv getestet. Molybdäntrioxid unterscheidet sich von Wismutoxid in zweierlei Hinsicht:

1. $\mathrm{MoO}_{3}$ hat mit $795^{\circ} \mathrm{C}$ einen niedrigeren Schmelzpunkt als Wismutoxid $\left(825^{\circ} \mathrm{C}\right)$.

2. Die Molybdänionen besitzen einen deutlich kleineren Ionenradius als das $\mathrm{Bi}^{3+}-$ Ion. Das Molybdän als Übergangsmetall besitzt Ionenradien, die mit den das Kristallgitter bildenden Kationen vergleichbar sind und kommt in allen Oxidationsstufen von $\mathrm{Mo}^{2+}$ bis $\mathrm{Mo}^{6+}$ vor. Es verursacht allerdings aufgrund seiner im Oxid bevorzugten Wertigkeit von $4+$ bzw. $6+{ }^{[i]}$ beim Einbau in das Kristallgitter Verzerrungen [92] (s. Tabelle 4.4).

\begin{tabular}{|c|c|c|c|c|c|c|c|c|}
\hline Ion & $\mathrm{Bi}^{3+}$ & $\mathrm{Mo}^{4+}$ & $\mathrm{Mo}^{6+}$ & $\mathrm{Fe}^{2+}$ & $\mathrm{Fe}^{3+}$ & $\mathrm{Mn}^{2+}$ & $\mathrm{Mn}^{3+}$ & $\mathrm{Zn}^{2+}$ \\
\hline Ionenradius [Á] & 0,96 & 0,70 & 0,62 & 0,74 & 0,64 & 0,80 & 0,66 & 0,74 \\
\hline
\end{tabular}

Tabelle 4.4 Vergleich der Ionenradien bei einigen relevanten Metallen [93].

Zugegeben wurden wieder Mengen von 0,02 Gew.\%, 0,04 Gew.\% und 0,06 Gew.\% (s. Übersicht über die Zusammensetzung der Ferritproben in Tabelle 3.1). Die Mikrostrukturuntersuchungen zeigen teilweise das Auftreten ähnlicher Effekte wie bei den mit

[i] Das vierwertige $\mathrm{MoO}_{2}$ oxidiert beim Erhitzen unter Luft und Sauerstoff zum sechswertigen, stabilen $\mathrm{MoO}_{3}$. 
Wismutoxid dotierten Proben. Es gibt jedoch auch Unterschiede. Abbildung 4.12 stellt die Gefüge nach dem Sintern unter Standardbedingungen $\left(1380^{\circ} \mathrm{C}, 12 \mathrm{~h}\right)$ dar.
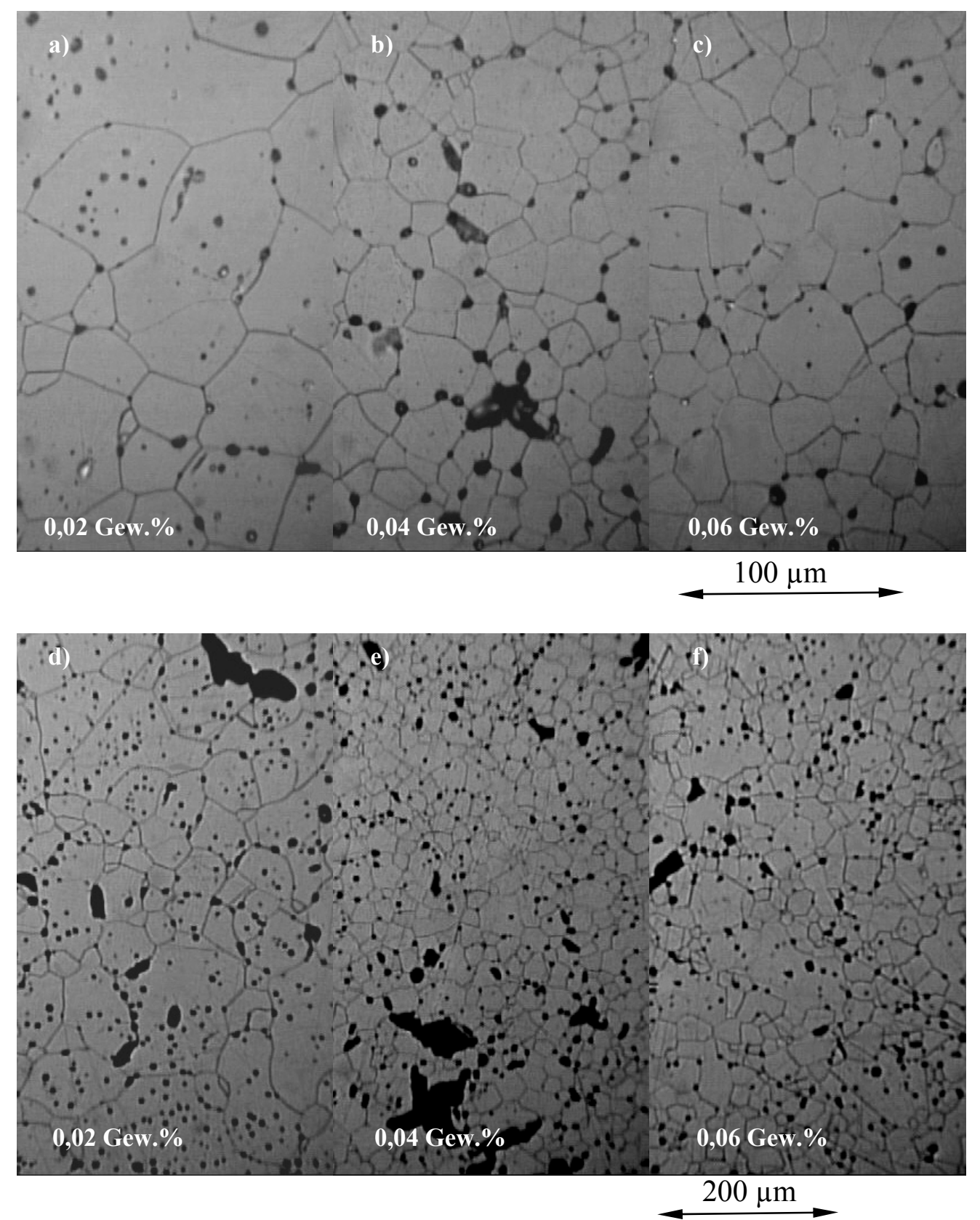

Abbildung 4.12 Änderung der Mikrostruktur bei den mit $\mathrm{MoO}_{3}$ dotierten Proben, gesintert mit einer Sintertemperatur von $1380^{\circ} \mathrm{C}$ und einer isothermen Haltezeit von $12 \mathrm{~h}$.

Im Vergleich fällt auf:

1. Schon bei der mit 0,02 Gew.\% vergleichsweise niedrig dotierten Probe beginnt diskontinuierliches Kornwachstum (bei $\mathrm{Bi}_{2} \mathrm{O}_{3}$ Dotierung erst ab 0,04 Gew.\%). Während sich bei den mit Wismutoxid dotierten Proben im Inneren sehr große Körner mit $\varnothing>1 \mathrm{~mm}$ ausbilden, beobachtet man bei der mit $0,02 \mathrm{Gew} \% \mathrm{MoO}_{3}$ dotierten 
Probe zwar das Losreißen einzelner Korngrenzen aus ihrer durch Poren gegebenen Verankerung, jedoch bleibt die Korngröße im Allgemeinen kleiner als $200 \mu \mathrm{m}$ (vgl. Abbildung 4.13 a)).

2. Es zeigt sich bei allen mit Molybdäntrioxid dotierten Proben eine deutliche Tendenz zur Porenkoaleszenz. Porenagglomerate bilden sich entweder während des Schwindungsintensivstadiums durch Umordnung einzelner Körner bzw. größerer Bereiche oder durch das Anwachsen interkristalliner Poren (Abbildung $4.13 \mathrm{~b}$ ) im Übergang zum Endstadium. Sie haben Abmessungen von bis zu $200 \mu \mathrm{m}$ und bauen sich auch durch langes isothermes Sintern nicht ab (vgl. Abschnitt 2.2.3).

3. Bei den Proben mit 0,04 Gew.\% und 0,06 Gew.\% Dotierung tritt diskontinuierliches Kornwachstum nicht in verstärktem Maße auf. Vielmehr verringert sich die mittlere Korngröße im Vergleich zur mit 0,02 Gew.\% dotierten Probe wieder.

a)

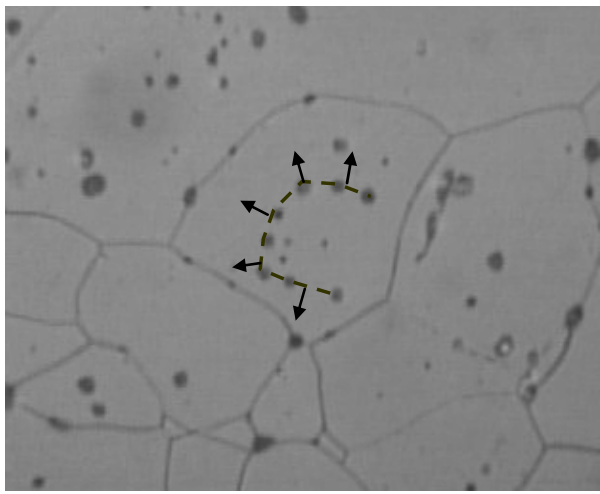

b)

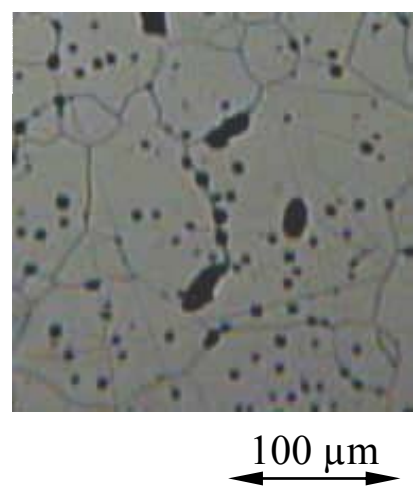

Abbildung 4.13 Ausschnitt aus Abbildung 4.12. Mikrostruktur der mit 0,02 Gew.\% $\mathrm{MoO}_{3}$ dotierten Proben. Teilabbildung a) zeigt eine Korngrenze, deren vermutete ursprüngliche Lage durch eine Reihe von Poren gekennzeichnet ist (gestrichelte Linie). Sie hat sich aus dieser Verankerung gelöst und hinterlässt bei ihrer Bewegung durch das umgebende Gefüge eine weitestgehend porenfreie Zone. Teilabbildung b) zeigt eine Porenagglomeration entlang einer Korngrenze.

In Abbildung 4.14 ist die Korngröße für die mit Wismut- und Molybdäntrioxid dotierten Proben nochmals im Vergleich aufgetragen. Interessant ist der Dotierungsbereich $\leq 0,03$ Gew.\%, da bei höheren Dotierungen das Gefüge wieder merklich feinkristalliner wird bzw. diskontinuierliches Kornwachstum auftritt. Es wird ersichtlich, dass geringe Mengen an Molybdäntrioxid das Kornwachstum stärker beschleunigen als Wismutoxid. Gleichzeitig nimmt die Streubreite der Korngrößenverteilung zu. 


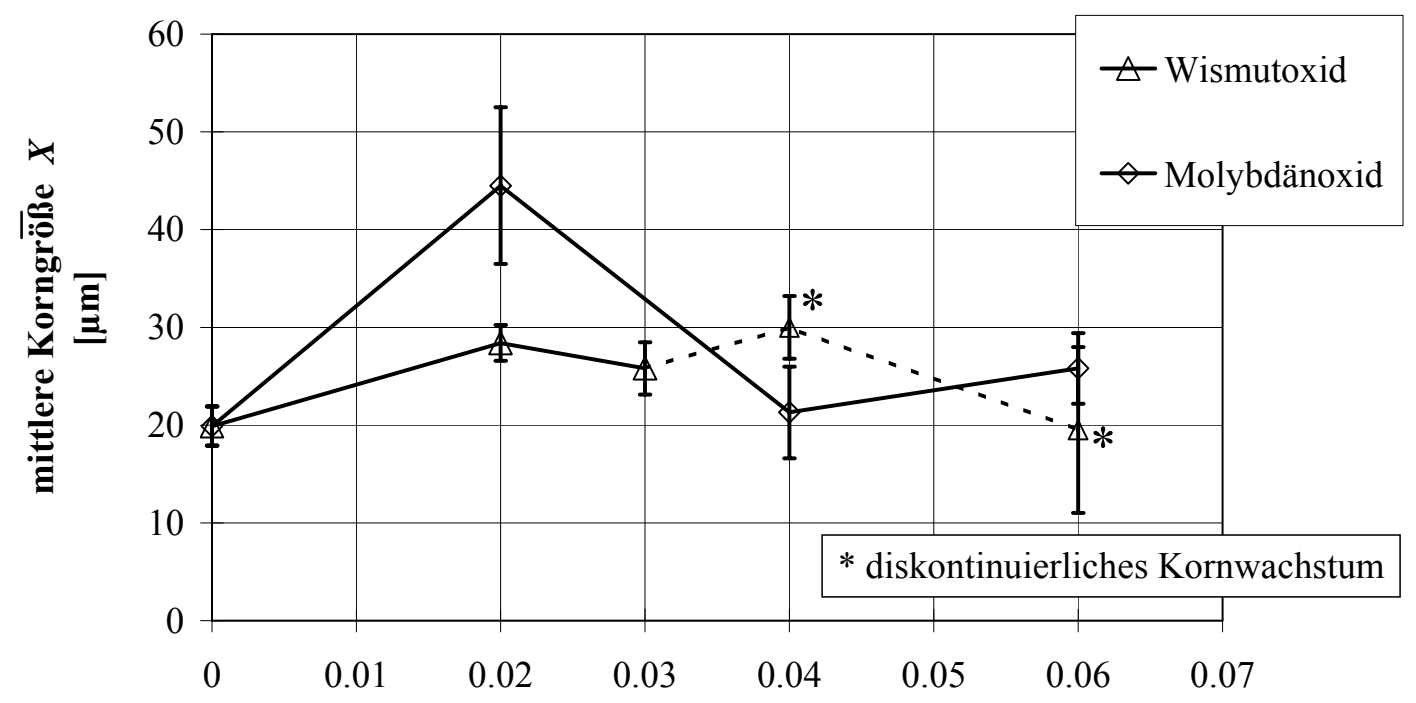

Gehalt in Gew.\%

Abbildung 4.14 Vergleich der Korngrößen für mit Wismut- und Molybdäntrioxid dotierte Proben. Bei den Proben mit Duplexstruktur wurden nur die kontinuierlich gewachsenen Gefügeanteile ausgewertet.

\begin{tabular}{c|c|c|l}
\hline $\begin{array}{c}\text { Probenbe- } \\
\text { zeichnung }\end{array}$ & $\begin{array}{c}\text { Gehalt an } \\
\mathrm{MoO}_{3} \\
{[\mathrm{Gew.} \mathrm{\% ]}}\end{array}$ & $\begin{array}{c}\text { Korngröße } \\
\bar{X} \\
{[\mu \mathrm{m}]}\end{array}$ & Charakterisierung des Gefüges \\
\hline HP1 & 0,00 & 19,9 & gleichmäßig, leicht bimodal \\
\hline HP1_Mo2 & 0,02 & 44,5 & $\begin{array}{l}\text { intragranulare Porosität, beginnendes diskonti- } \\
\text { nuierliches Kornwachstum, agglomerierte Poren }\end{array}$ \\
\hline HP1_Mo4 & 0,04 & 21,3 & $\begin{array}{l}\text { hohe intergranulare Porosität, zum Teil } \\
\text { zusammenhängende Porenagglomerate, geringere } \\
\text { intragranulare Porosität }\end{array}$ \\
\hline HP1_Mo6 & 0,06 & 25,8 & wie bei HP1_Mo4 \\
\hline
\end{tabular}

Tabelle 4.5 Übersicht über die Änderung der Gefügeeigenschaften bei Molybdäntrioxidzugabe. Die isotherme Haltezeit beträgt bei allen untersuchten Gefügen $12 \mathrm{~h}$, die Sintertemperatur $1380^{\circ} \mathrm{C}$. Eine Bestimmung des Porenanteils war wegen der ungleichmäßig verteilten Agglomerate nicht möglich. 


\subsubsection{Transmissionselektronenmikroskopische Untersuchungen (TEM)}

Alle TEM - Untersuchungen wurden an speziell dafür hergestellten Proben mit einem Durchmesser von $3 \mathrm{~mm}$ durchgeführt. Folglich befinden sich die untersuchten Stellen in der Regel in einem Abstand von ca. 1,5 mm vom Probenrand (vgl. Abschnitt 3.5.2). Besondere Aufmerksamkeit gilt den Korngrenzen, die sich in zwei Kategorien unterteilen lassen:

1. Korngrenzen ohne intergranulare Phase: Auch bei höchster Auflösung ist es nicht möglich, eine segregierte Phase zwischen zwei Körnern nachzuweisen.

2. Korngrenze mit intergranularer Phase: Zwischen den Körnern befindet sich eine segregierte Phase mit einer Breite von 10 - $250 \mathrm{~nm}$.

Folgende Ergebnisse liegen vor:

\section{Proben ohne Wismutdotierung}

Ungefähr jede dritte der untersuchten Korngrenzen zeigt eine intergranulare Phase. Die Breite liegt bei ca. $15-35 \mathrm{~nm}$ (Abbildung 4.15 a) und b)). Die in den Korngrenzen mittels EDX-Analyse gemessenen Elementverteilungen bewegen sich im Zusammensetzungsbereich der angrenzenden Körner (Abbildung 4.16). Der Sauerstoffgehalt konnte nicht analysiert werden ${ }^{[\mathrm{i}]}$.

Innerhalb der Nachweisgrenzen von ca. 0,1 Gew.\% ließen sich in den Korngrenzen keine Fremdatome, wie z.B. das in den Rohstoffen als Verunreinigung enthaltene Kalzium oder Silizium (vgl. Abschnitt 3.2.1), nachweisen. Eine genauere Betrachtung der EDXAnalyse lässt allenfalls Spuren von Kalzium vermuten. Dies kann an der durch den Strahldurchmesser von ca. 20 - $30 \mathrm{~nm}$ limitierten Ortsauflösung liegen, die zusammen mit den häufig nicht ganz senkrecht verlaufenden Korngrenzen dazu führt, dass auch Bereiche der benachbarten Körner zur Emission angeregt werden und somit die Analyse verfälschen.

Ein Vergleich der Analyseergebnisse mit der aus der Einwaage berechneten Zusammensetzung ergibt einen Fehler von ca. 2,0 Gew.\% bei der Bestimmung des Eisenanteils. Dies liegt ungefähr im Rahmen der Analysegenauigkeit des EDX-Systems, die durch Wechselwirkungen mit der umgebenden Apparatur des Elektronenmikroskops reduziert wird.

[i] Es ist durchaus denkbar, dass sich aufgrund unterschiedlicher Diffusionsbedingungen der Oxidationsgrad des Ferrits in der Korngrenze von dem Oxidationsgrad im Inneren der Körner unterscheidet. 
Die Breite der Kornzwischenphasen variiert von $10 \mathrm{~nm}$ bis $\mathrm{zu} 50 \mathrm{~nm}$. Im Beugungsmodus lässt sich die Struktur der Zwischenphase nicht auflösen. Es ist beim Überstreichen der Korngrenze jeweils nur ein Übergang der Beugungsbilder der benachbarten Körner sichtbar. Insbesondere konnte keine amorphe Struktur nachgewiesen werden.

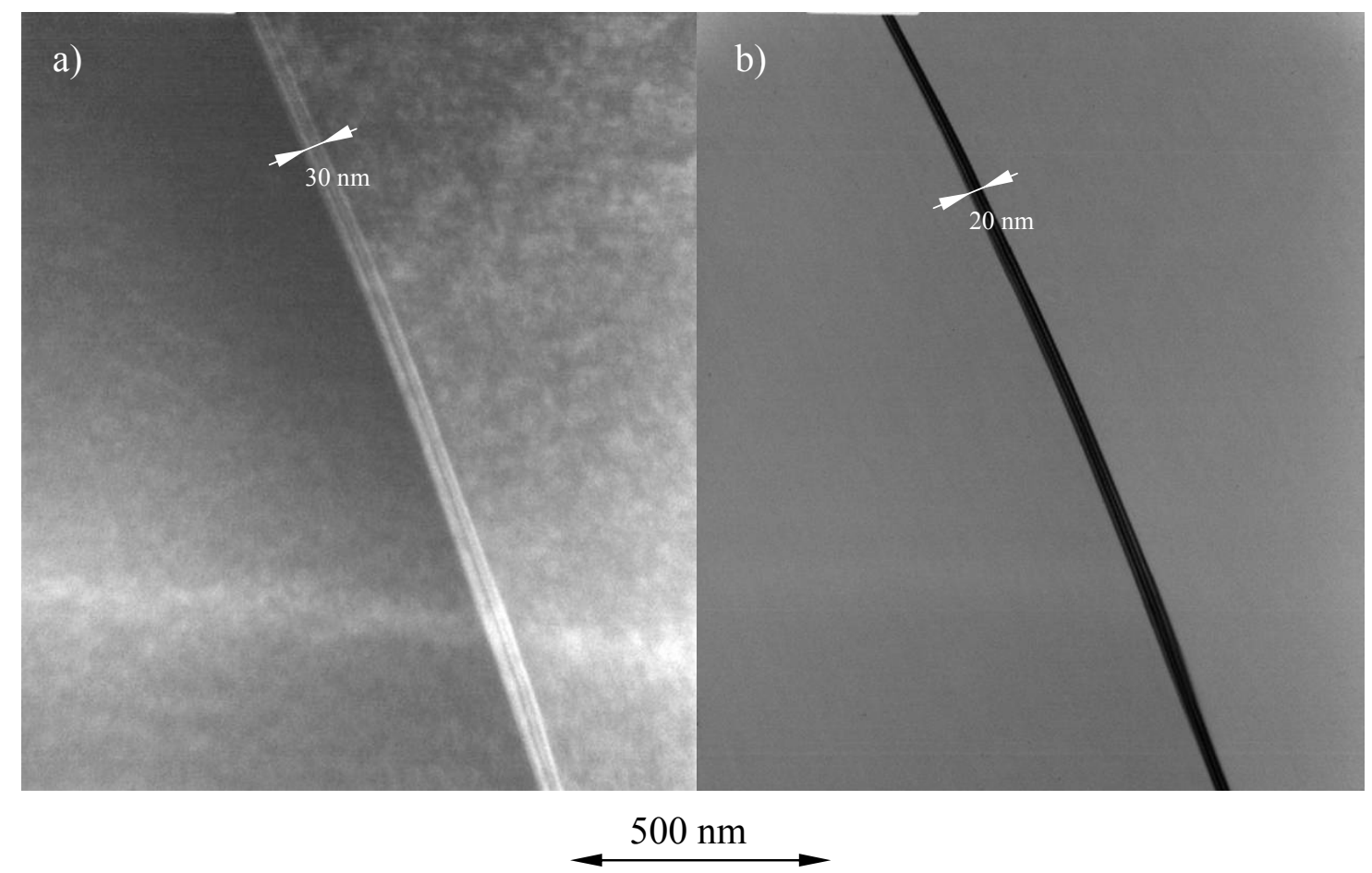

Abbildung 4.15 Typische Korngrenze mit segregierter Zwischenphase in einer undotierten Probe (HP1).

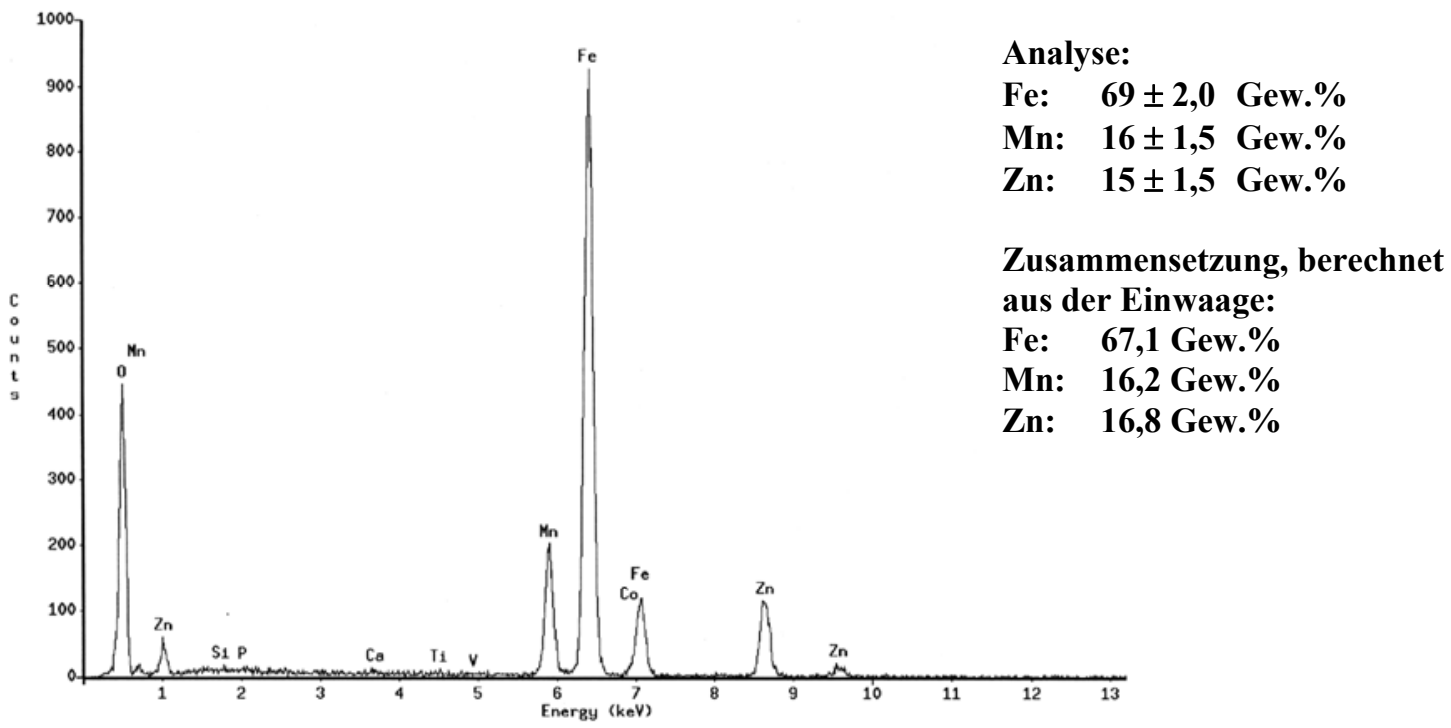

Abbildung 4.16 EDX - Analyse einer Kornzwischenphase der undotierten Probe HP1. Der vorhandene Sauerstoff ist nicht berücksichtigt. 


\section{Proben mit 0,03 Gew.\% Wismutoxid}

Obwohl bei diesen Proben noch keine diskontinuierlich gewachsenen Körner vorliegen, ist die mechanische Stabilität im Vergleich zu den undotierten Proben deutlich reduziert. Beide Typen von Korngrenzen sind auch hier vorhanden. Wieder lässt sich in ungefähr jeder dritten Korngrenze eine segregierte Phase finden. Diese spalten sich jedoch in zwei Typen auf. Der seltener vorkommende Typ 1 ähnelt den bei den undotierten Proben vorkommenden Zwischenphasen.

Typ 2 hat eine andere Erscheinung und andere Eigenschaften. Zum einen ist bei diesem Typ die Breite der Phase deutlich erhöht und liegt teilweise über $200 \mathrm{~nm}$, zum anderen sind die intergranularen Phasen sehr brüchig. Teilweise finden sich Risse in der Phase (Abbildung 4.17) oder die angrenzenden Körner sind während der Probenpräparation ausgebrochen. Die schlechten mechanischen Eigenschaften lassen sich also auf eine geringere Stabilität der Korngrenzen zurückführen.

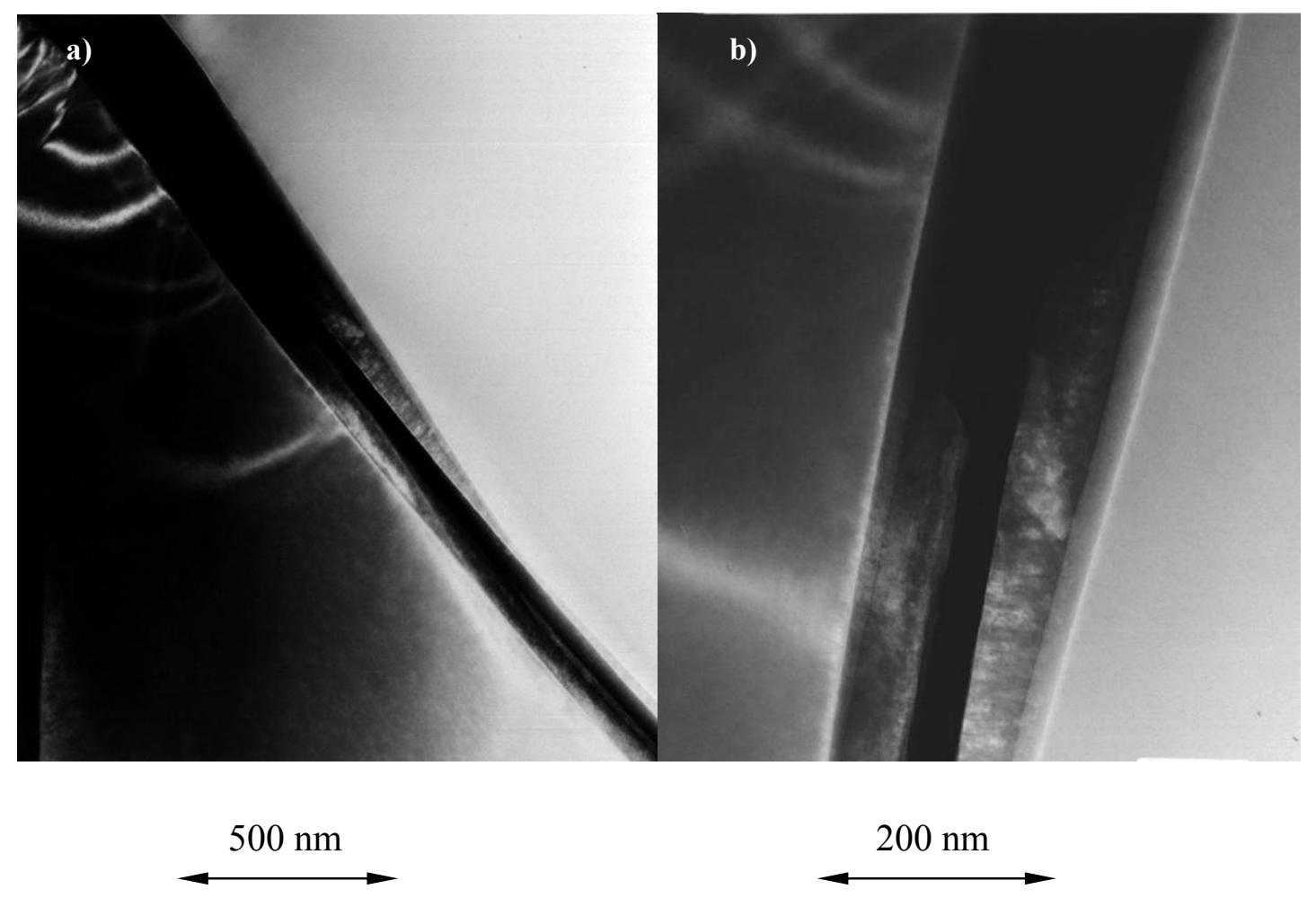

Abbildung 4.17 Kornzwischenphase der mit 0,03 Gew.\% $\mathrm{Bi}_{2} \mathrm{O}_{3}$ dotierten Probe HP1_Bi3 vom Typ 2. Durch die Korngrenze verläuft entweder ein Riss oder Teile der intergranularen Phase sind während der Probenpräparation ausgebrochen [89].

Die Röntgen-Analyse (EDX) der intergranularen Phasen vom Typ 2 zeigt deutlich erhöhte Anteile an Kalzium bzw. Silizium. Allerdings kommen die beiden aus den verwendeten Rohstoffen stammenden Bestandteile in sehr unterschiedlichen Verhältnissen vor. Meistens ist einer der beiden Bestandteile dominant. In Abbildung 4.18 ist eine Anreicherung von Kalzium, in Abbildung 4.19 eine starke Anreicherung von 
Silizium erkennbar. In beiden Fällen handelt es sich mit Sicherheit um oxidische Ausscheidungen, der Sauerstoffgehalt ließ sich wegen der geringen Energie der Röntgenstrahlung nicht bestimmen.

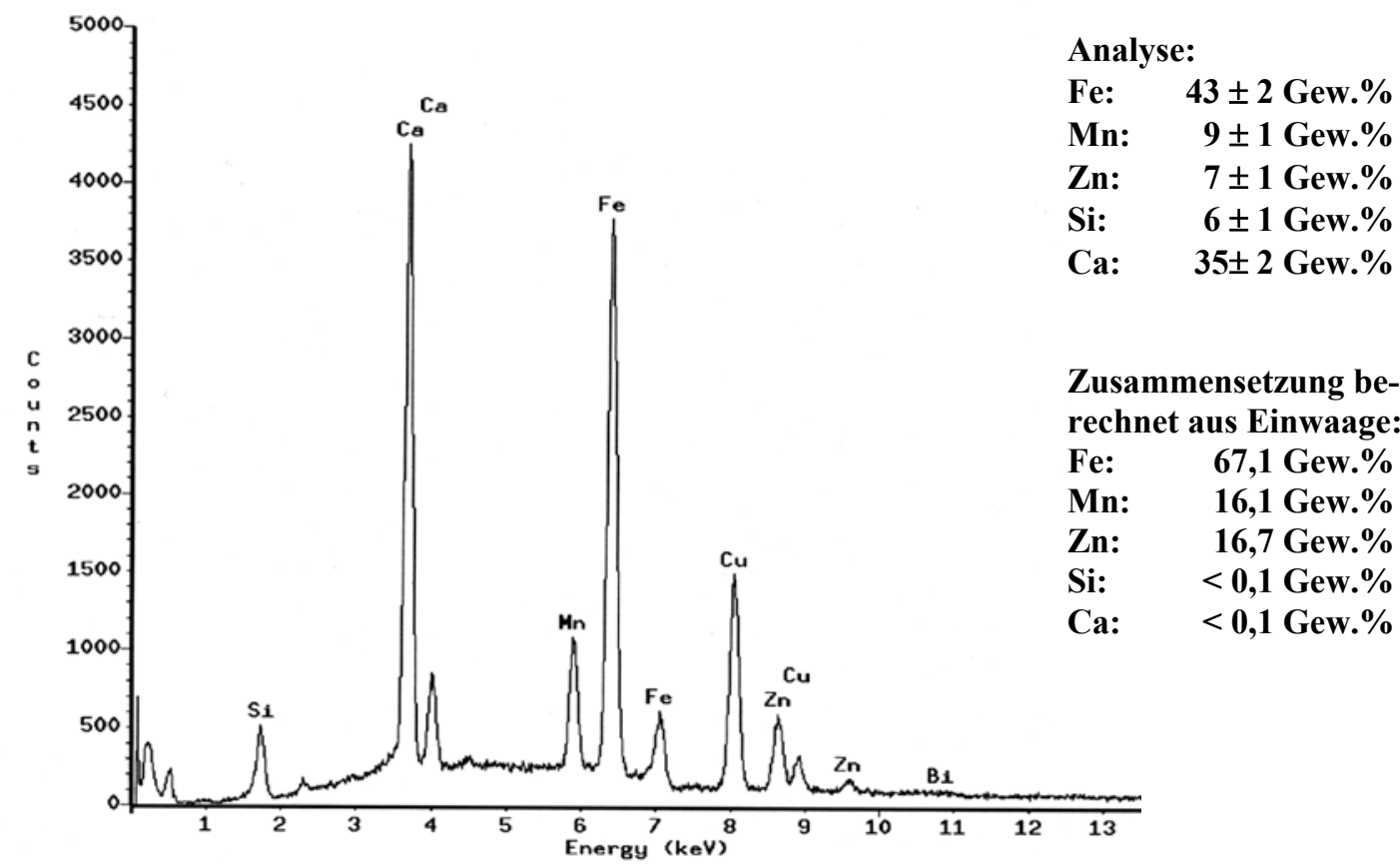

Abbildung 4.18 EDX - Analyse einer Korngrenze mit zersplitterter Zwischenphase. Eine deutlich erhöhte Kalzium- und Siliziumkonzentration ist sichtbar. Es konnte kein Wismut nachgewiesen werden.

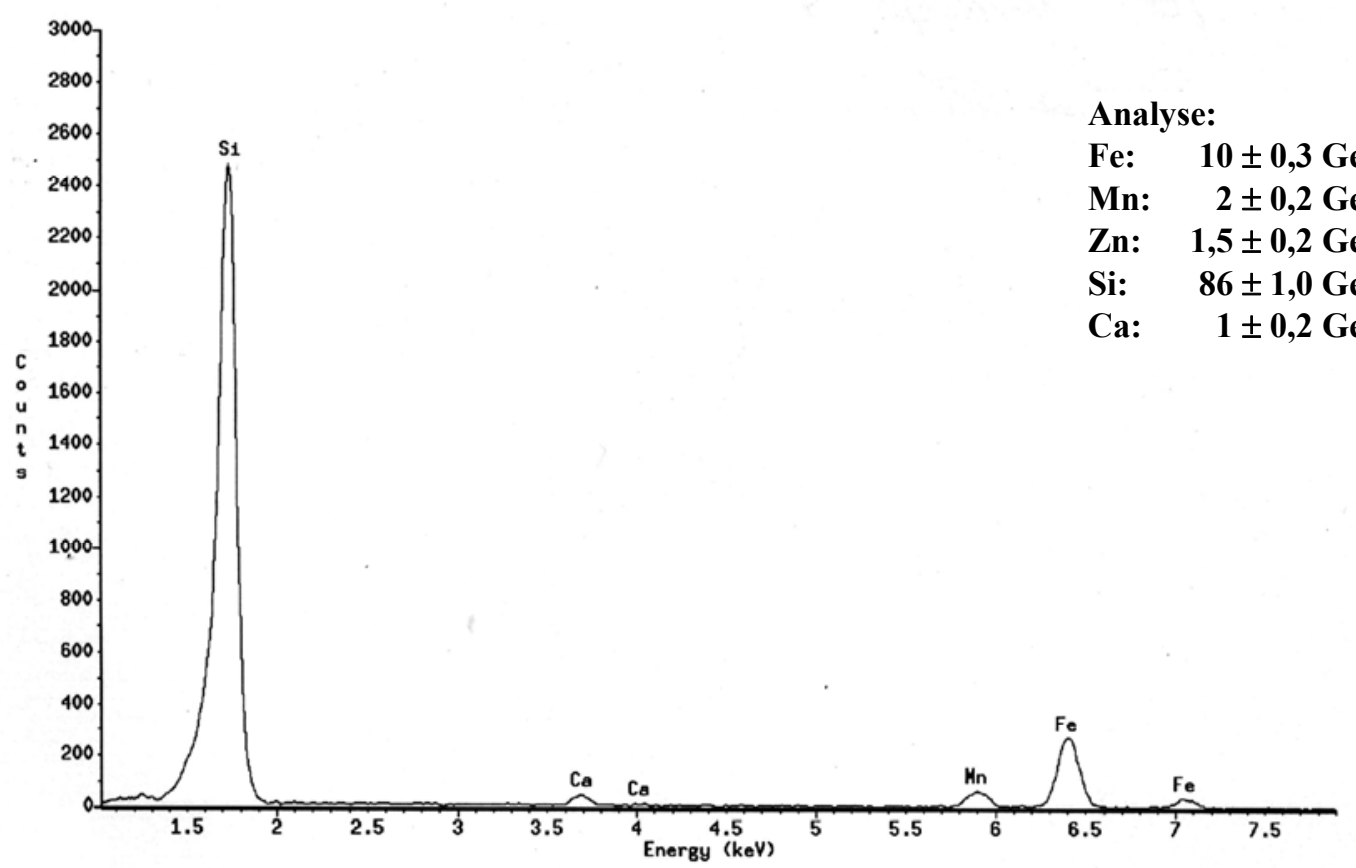

Abbildung 4.19 EDX - Analyse eines Siliziumsplitters aus einer intergranularen Segregation. Der Siliziumgehalt beträgt ohne Berücksichtigung des Sauerstoffs 85 Gew.\%. 
Beachtenswert ist insbesondere die siliziumreiche Ausscheidung. Die Analyse wurde an einem Stück intergranularer Phase durchgeführt, welches sich direkt neben einem bei der Präparation ausgebrochenen Bereich befindet. Die häufig auftretenden Störungen durch benachbarte bzw. schräg unter dem fokussierten Bereich liegende Körner ließen sich aufgrund dieser isolierten Position vermeiden. Die untersuchte intergranulare Phase besteht aus nahezu reinem Siliziumoxid.

Ein weiteres wichtiges Ergebnis ist der fehlende Nachweis von Wismut. Es ist davon auszugehen, dass aufgrund der kleinen Probenabmessungen $(\varnothing=3 \mathrm{~mm})$ das Wismutoxid beim Sintern des Materials größtenteils verdampft (vgl. Abschnitt 4.1 .5 und [70]). Fremdatome und Verunreinigungen lösen sich verstärkt in der Wismutoxidschmelze und verbleiben als interkristalline Ausscheidung nach dem Verdampfen des Wismutoxids. Es kommt also zur Anreicherung von Fremdatomen in den breiten, vormals verflüssigten Kornzwischenphasen. Korngrenzen ohne Schmelze und das Kristallgitter werden von Fremdatomen gereinigt.

\subsubsection{Röntgenfluoreszenzanalyse (RFA)}

An den mit Wismutoxid dotierten Proben wurden Röntgenfluoreszenzmessungen durchgeführt um das Abdampfen des Wismutoxids während des Sinterns zu belegen.

Gemessen wird der mittlere $\mathrm{Bi}_{2} \mathrm{O}_{3}$-Gehalt der Proben. Die ungesinterten Proben dienten hierbei zur Berechnung der Kalibriergeraden (durchgezogene Linie in Abbildung 4.20).

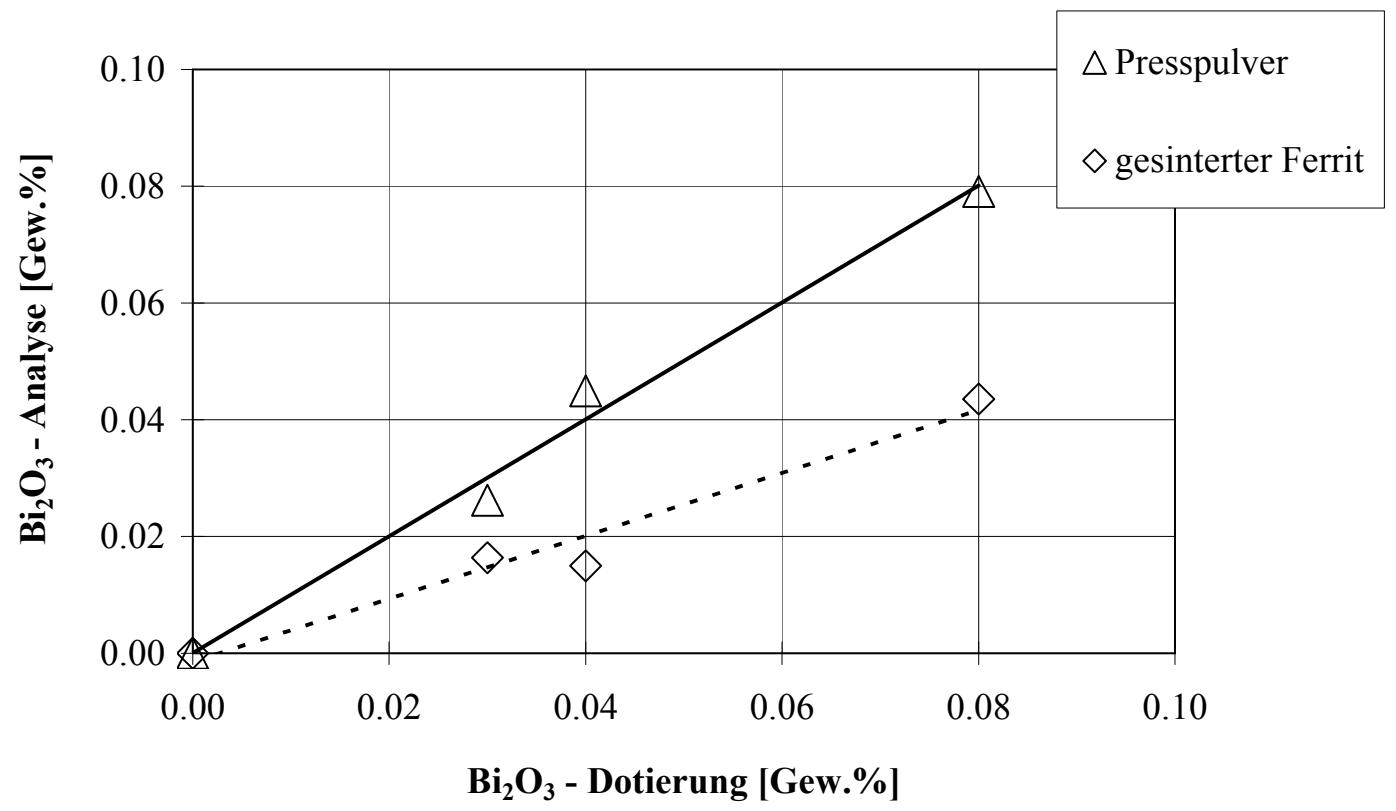

Abbildung 4.20 Vergleich der Zusammensetzungen zwischen keramischem Pulver und gesintertem Ferrit. 
Bei den untersuchten Ringen mit einer Wandstärke von $3 \mathrm{~mm}$ ist nach der Standardtemperaturbehandlung von $1380{ }^{\circ} \mathrm{C} / 12 \mathrm{~h}$ ungefähr die Hälfte des Wismutoxids abgedampft. Bei den für die TEM-Untersuchungen hergestellten Stäben mit $\varnothing 3 \mathrm{~mm}$ ist dieser Anteil wahrscheinlich noch höher.

\subsubsection{Messung der magnetischen Eigenschaften}

Sowohl die Wahl des schmelzfließenden Additivs als auch die Menge an schmelzfließender Phase beeinflussen das Sinterverhalten und damit das Gefüge des Ferritmaterials stark. Es ist zu erwarten, dass sich die vorhandenen Gefügeunterschiede in den elektromagnetischen Eigenschaften widerspiegeln.

\section{Anfangspermeabilität $\underline{\mu}_{i}$}

Die Anfangspermeabilität $\mu_{i}$ ist einer der wichtigsten magnetischen Parameter zur Charakterisierung hochpermeabler MnZn-Ferrite (vgl. Abschnitt 2.1.7). In Abbildung 4.21 ist ihr Verlauf in Abhängigkeit vom Gehalt an Wismut- bzw. Molybdäntrioxid dargestellt. Bei beiden Systemen erkennt man schon bei geringen Zusätzen einen merklichen Anstieg in der Anfangspermeabilität und einen darauf folgenden Abfall bei weiterer Zugabe der Dotierung. Das Maximum liegt in beiden Fällen bei ca. 0,2 Gew.\%.

Die Verläufe für die beiden Additive gleichen sich, obwohl sich das Gefüge, wie im vorigen Abschnitt beschrieben, mit Zunahme der Dotierung sehr unterschiedlich entwickelt.

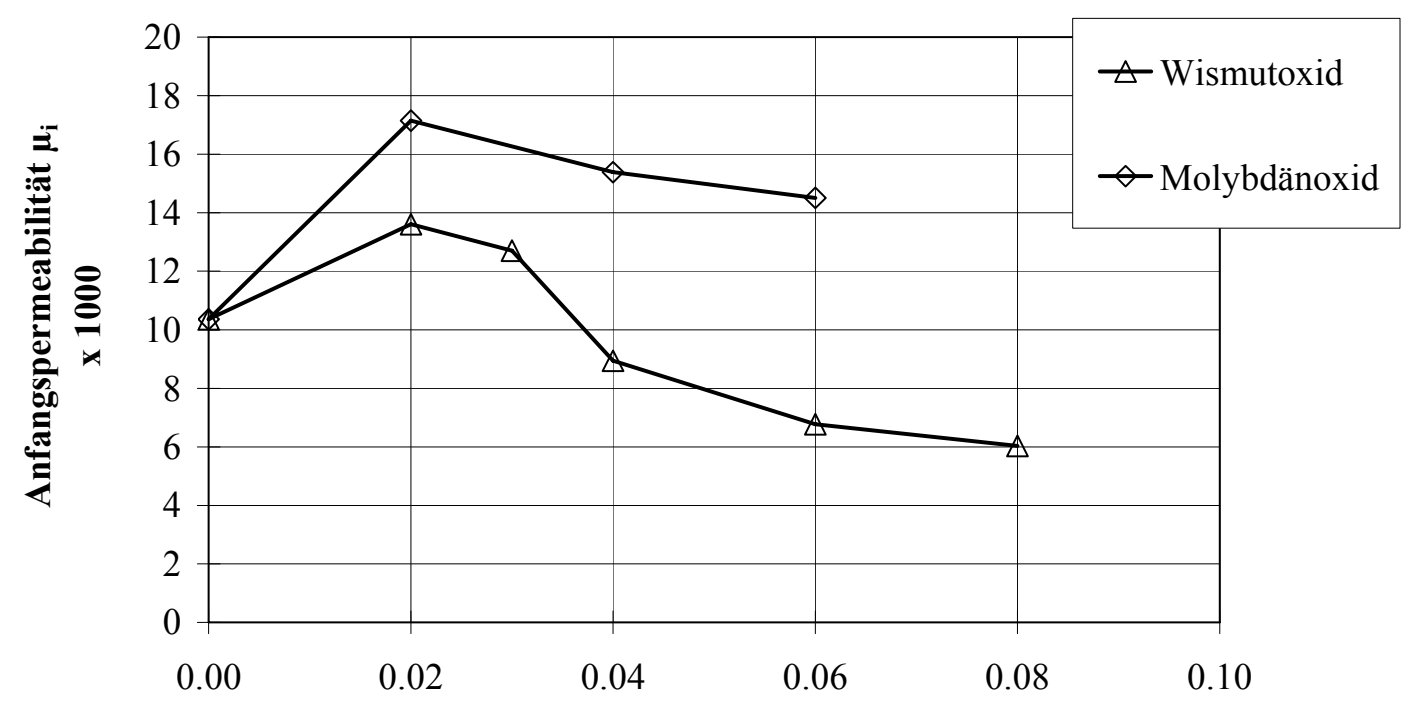

Gehalt in Gew.\%

Abbildung 4.21 Änderung der Anfangspermeabilität mit dem Gehalt an Molybdän- bzw. Wismutoxid. 
Es ist daher sinnvoll, die Permeabilität in Relation zu messbaren Gefügemerkmalen zu setzen. In [29] wird in Übereinstimmung mit dem Modell von Globus [27], eine proportionale Zunahme der Anfangspermeabilität mit der Korngröße beschrieben (vgl. 2.1.7.) Die hierbei untersuchten Gefüge sind weitestgehend porenfrei und zeigen kein diskontinuierliches Kornwachstum. Abbildung 4.22 zeigt diesen linearen Zusammenhang für die $\mathrm{Bi}_{2} \mathrm{O}_{3}$-dotierten Proben, bei denen kein diskontinuierliches Kornwachstum auftritt. Bei den $\mathrm{MoO}_{3}$-dotierten Proben mit ihrer hohen Porosität und Neigung zur Porenkoaleszenz ist ein ähnlicher Zusammenhang jedoch nicht mehr gegeben.

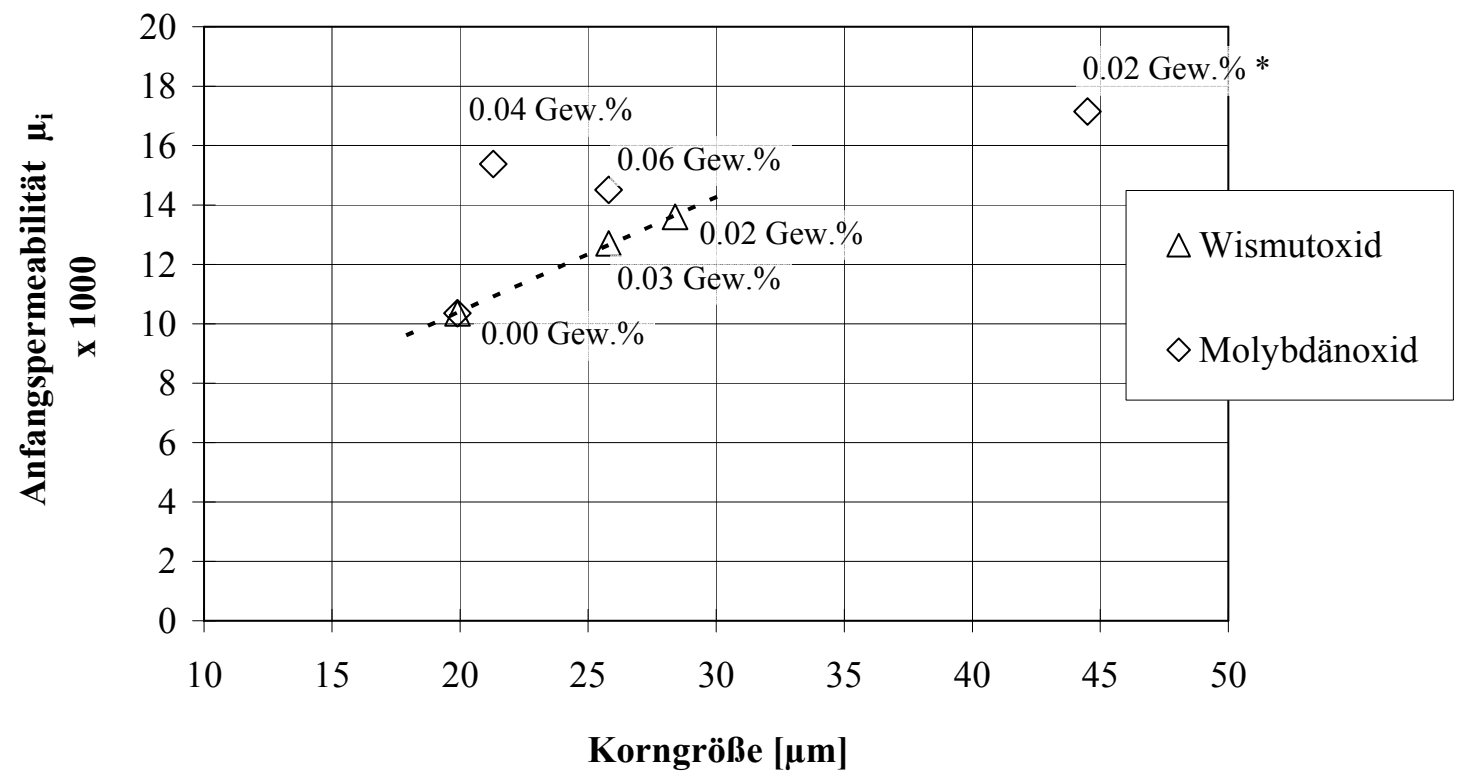

\footnotetext{
Abbildung 4.22 Zusammenhang zwischen Korngröße und Permeabilität für kontinuierliches Kornwachstum. Bei dem mit * gekennzeichneten Messpunkt findet der Übergang zum diskontinuierlichen Kornwachstum statt.
}

Es liegt daher nahe, den Einfluss der Porosität zu untersuchen. Unabhängig von der Korngröße bzw. vom Auftreten diskontinuierlichen Kornwachstums zeigt Abbildung 4.23 eine weitestgehend lineare Abhängigkeit der Permeabilität von der Porosität bei den mit Wismutoxid dotierten Proben. Die Porosität der Proben mit Molybdäntrioxiddotierung konnte wegen der oben erwähnten ungleichmäßigen Porenverteilung und dem Auftreten von Porenagglomeraten nicht bestimmt werden. 


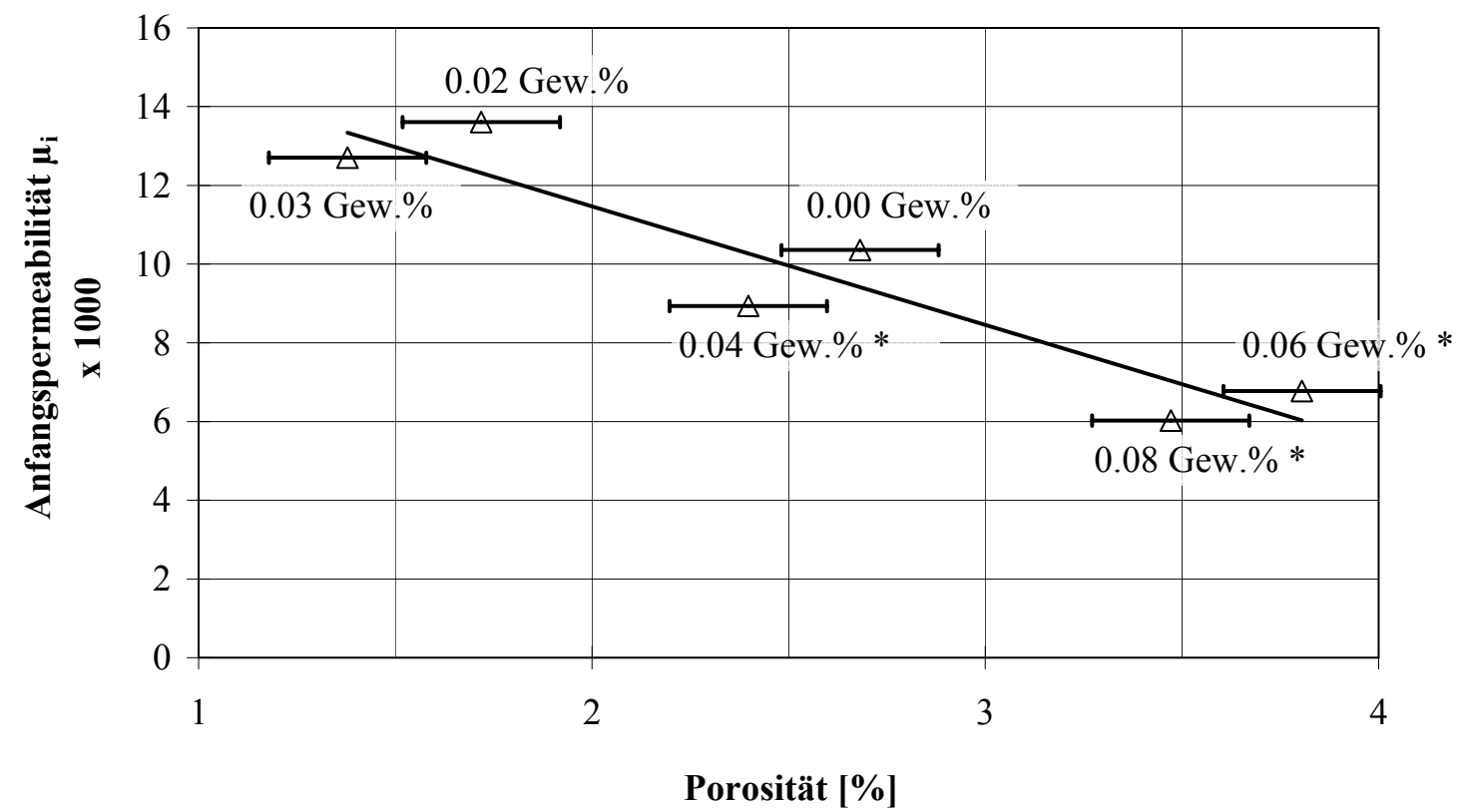

Abbildung 4.23 Abhängigkeit der Anfangspermeabilität von der Porosität für die mit Wismutoxid
dotierte Probenreihe. Bei den mit * gekennzeichneten Proben tritt diskontinuier-
liches Kornwachstum auf.

\subsubsection{Messung weiterer magnetischer Eigenschaften}

Die Messung weiterer magnetischer Parameter wie der Sättigungsmagnetisierung, der Remanenz und der Koerzitivfeldstärke bestätigen die in Abschnitt 2.1.7 beschriebenen bekannten Zusammenhänge zwischen Mikrostruktur und Magnetismus.

Die Sättigungsmagnetisierung passt gut zu den gemessenen Dichten und Porositäten, die Koerzitivfeldstärke und Remanenz sind aufgrund von Blochwand-Pinning-Effekten insbesondere bei den Proben mit hoher intrakristalliner Porosität erhöht. 


\begin{tabular}{c|c|c|c|c|c}
\hline Probe & $\begin{array}{c}\text { Magneti- } \\
\text { sierung } \\
\text { Bsat [mT] }\end{array}$ & $\begin{array}{c}\text { Koerzitiv- } \\
\text { feldstärke } \\
\text { Hc }[\mathrm{A} / \mathrm{m}]\end{array}$ & $\begin{array}{c}\text { Remanenz } \\
\mathrm{Br}[\mathrm{mT}]\end{array}$ & $\begin{array}{c}\text { Porosität } \\
\Theta \\
{[\%]}\end{array}$ & $\begin{array}{c}\text { Korngröße } \\
\bar{X} \\
{[\mu \mathrm{m}]}\end{array}$ \\
\hline HP1 & 461 & 7,9 & 221 & 2,7 & 19,9 \\
\hline HP1_Bi2 & 472 & 8,0 & 205 & 1,7 & 28,4 \\
\hline HP1_Bi3 & 471 & 8,2 & 207 & 1,4 & 25,8 \\
\hline HP1_Bi4 & 452 & 10,7 & 248 & 2,4 & $\begin{array}{c}>1000 \\
\text { bzw. } 29,7\end{array}$ \\
\hline HP1_Bi6 & 451 & 11,6 & 258 & 3,8 & $>1000$ \\
\hline HP1_Mo6 & 455 & 7,9 & 221 & - & bzw. 19,2 \\
\hline HP1_Mo2 & 458 & 6,1 & 224 & 21,3 \\
\hline
\end{tabular}

Tabelle 4.6 $\quad$ Sättigungsmagnetisierung, Koerzitivfeldstärke und Remanenz im Vergleich zu Korngröße und Porosität. Bei den mit Molybdäntrioxid dotierten Proben konnte die Porosität wegen des Auftretens ungleichmäßiger Porenagglomerate nicht bestimmt werden (vgl. die Abschnitte 4.1.2 und 4.1.3). 


\subsection{Gefügeoptimierung durch Elektro - Mikrowellen - Hybridsintern}

Der zweite Komplex der durchgeführten Untersuchungen betrifft den Einsatz neuer, innovativer Sintertechnik. Bei den im vorigen Abschnitt untersuchten hochpermeablen MnZn-Ferrite ist ein vergleichsweise grobkristallines Gefüge vorteilhaft. Für einige Anwendungen ist jedoch umgekehrt ein möglichst feinkristallines Gefüge günstiger. Dies sind z.B. MnZn-Ferrite, die zum Übertragen hoher Leistungen bei Aussteuerungen von $50-200 \mathrm{mT}$ und Arbeitsfrequenzen $>300 \mathrm{kHz}$ eingesetzt werden (vgl. Abschnitt 2.1.7). Eine Strategie zur Herstellung feinkristalliner Gefüge ist eine schnelle Verdichtung, die nach Möglichkeit schon mit Erreichen der Maximaltemperatur abgeschlossen ist, eine kurze Haltezeit, in der es zu keinem nennenswerten Kornwachstum kommt und ein schnelles Abkühlen der Proben unter Beachtung der Gleichgewichtsbedingungen. Für einen solchen Sinterzyklus bietet sich das bisher nur unzureichend und an eher kleinen Ofenvolumina erprobte Konzept des Mikrowellensinterns an [94].

\subsubsection{Vergleich mit konventioneller Sinterung}

Erste Versuche wurden mit NiZn-Ferriten der Zusammensetzung Ni1 durchgeführt. NiZn-Ferrite benötigen im Gegensatz zu den MnZn-Ferriten beim Abkühlen keine Regelung des Sauerstoffpartialdruckes und können unter Luft-Atmosphäre gesintert werden (vgl. Abschnitt 3.1.2 und 3.2.2).

Bei den gesinterten Ferritproben handelt es sich um dünnwandige Ringe mit den Abmessungen $\varnothing_{\text {außen }} \times \varnothing_{\text {innen }} \times \mathrm{h} \approx 8 \mathrm{~mm}$ x 5,3 mm x $10 \mathrm{~mm}$. Die Sinterzyklen sind sehr kurz, ein kalt-kalt-Zyklus hat eine Dauer von 200 min. Allerdings müssen im Vorfeld die organischen Bestandteile separat ausgebrannt werden, weshalb sich die Sinterdauer für einen direkten Vergleich nochmals um 150 min verlängert. In Tabelle 4.7 werden drei unterschiedliche Temperaturprofile bzw. Sintertechnologien miteinander verglichen. Dies sind a) ein konventioneller Sinterzyklus mit reiner Widerstandsbeheizung und niedrigen Aufheizraten von ca. $5 \mathrm{~K} / \mathrm{min}$ und langer Sinterdauer, b) ein Sinterzyklus mit kombinierter Widerstands-Mikrowellen-Beheizung mit einem Mikrowellenanteil von $40 \%$ und c) ein Sinterzyklus mit reiner Widerstandsbeheizung und hohen Aufheizraten von $25-30 \mathrm{~K} / \mathrm{min}$. Die Sintertemperatur wurde bei den Proben b) und c) bewusst höher gewählt, um die verkürzte Sinterzeit $\mathrm{zu}$ kompensieren und eine vergleichbare Anfangspermeabilität von ca. $1500 \mathrm{zu}$ erreichen. Mit der konventionellen 
Widerstandsbeheizung gelingt dies mit einem verhältnismäßig langen Sinterzyklus (1060 min).

\begin{tabular}{l|c|c|c}
\hline Eigenschaft & $\begin{array}{l}\text { a) Widerstands- } \\
\text { beheizung, niedrige } \\
\text { Aufheizrate }\end{array}$ & $\begin{array}{l}\text { b) Elektro- } \\
\text { Mikrowellen- } \\
\text { Hybridsinterofen }\end{array}$ & $\begin{array}{l}\text { c) Widerstands- } \\
\text { beheizung, hohe } \\
\text { Aufheizraten }\end{array}$ \\
\hline Ofenvolumen & $0,63 \mathrm{~m}^{3}(\mathrm{vgl} .3 .3 .1)$ & $0,01 \mathrm{~m}^{3}(\mathrm{vgl} .3 .3 .2)$ & $0,01 \mathrm{~m}^{3}(\mathrm{vgl} .3 .3 .2)$ \\
\hline Heizung & Widerstandsbeheizung & $\begin{array}{l}\text { Widerstands }-\mathrm{MW}- \\
\text { Beheizung } 40 \% \\
\mathrm{MW}\end{array}$ & Widerstandsbeheizung \\
\hline Aufheizrate & $4-7 \mathrm{~K} / \mathrm{min}$ & $25 \mathrm{~K} / \mathrm{min}$ & $25 \mathrm{~K} / \mathrm{min}$ \\
\hline isotherme Haltezeit & $500 \mathrm{~min}$ & $30 \mathrm{~min}$ & $30 \mathrm{~min}$ \\
\hline $\begin{array}{l}\text { isotherme } \\
\text { Sintertemperatur }\end{array}$ & $1200{ }^{\circ} \mathrm{C}$ & $1250{ }^{\circ} \mathrm{C}$ & $1250{ }^{\circ} \mathrm{C}$ \\
\hline $\begin{array}{l}\text { Länge des Ofenzyklus } \\
\text { kalt-kalt) }\end{array}$ & $1060 \mathrm{~min}$ & $\begin{array}{c}200 \mathrm{~min} \\
+150 \mathrm{~min} \mathrm{Entbind.}\end{array}$ & $+150 \mathrm{~min} \mathrm{Entbind.}$ \\
\hline $\begin{array}{l}\text { Dichte der Ferritproben } \\
\text { Korngröße: }\end{array}$ & $4,8 \mathrm{~g} / \mathrm{cm}^{3}$ & $5,2 \mathrm{~g} / \mathrm{cm}^{3}$ & $5,2 \mathrm{~g} / \mathrm{cm}^{3}$ \\
\hline $\begin{array}{l}\text { Anfangspermeabilität } \mu_{i} \\
\text { der Proben bei } 10 \mathrm{kHz}\end{array}$ & $10-50 \mu \mathrm{m}$ & $<10 \mu \mathrm{m}$ & $<15 \mu \mathrm{m}$ \\
\hline $\begin{array}{l}\text { bezogener Verlustfaktor } \\
\text { tan } \delta / \mu_{\mathrm{i}} \mathrm{x} 10^{-6} \text { bei } \\
500 \mathrm{kHz}\end{array}$ & 1500 & 1700 & 1340 \\
\hline
\end{tabular}

Tabelle 4.7 Vergleich zwischen drei unterschiedlichen Sinterzyklen.

Bei Einsatz der Mikrowellen-Hybridsintertechnik werden vergleichbare bzw. sogar leicht verbesserte Eigenschaften mit einer um $67 \%$ verkürzten Sinterdauer erreicht. Die Permeabilität der Proben ist sogar leicht erhöht und der bezogene Verlustfaktor $\tan \delta / \mu_{\mathrm{i}}$ um ca. $23 \%$ erniedrigt. Diese Eigenschaftsverbesserung geht mit einer Zunahme der Enddichte einher.

Im Vergleich hierzu zeigt die mit dem ebenfalls verkürzten Sinterzyklus c) von außen erwärmte Probe zwar ein ähnlich feinkristallines Gefüge, jedoch sind die elektrischen Werte verschlechtert. Die Anfangspermeabilität ist mit 1340 niedriger, der Verlustfaktor und die Enddichte ähnlich wie bei den mit Mikrowelle gesinterten Proben. 


\section{Gefügeuntersuchungen}
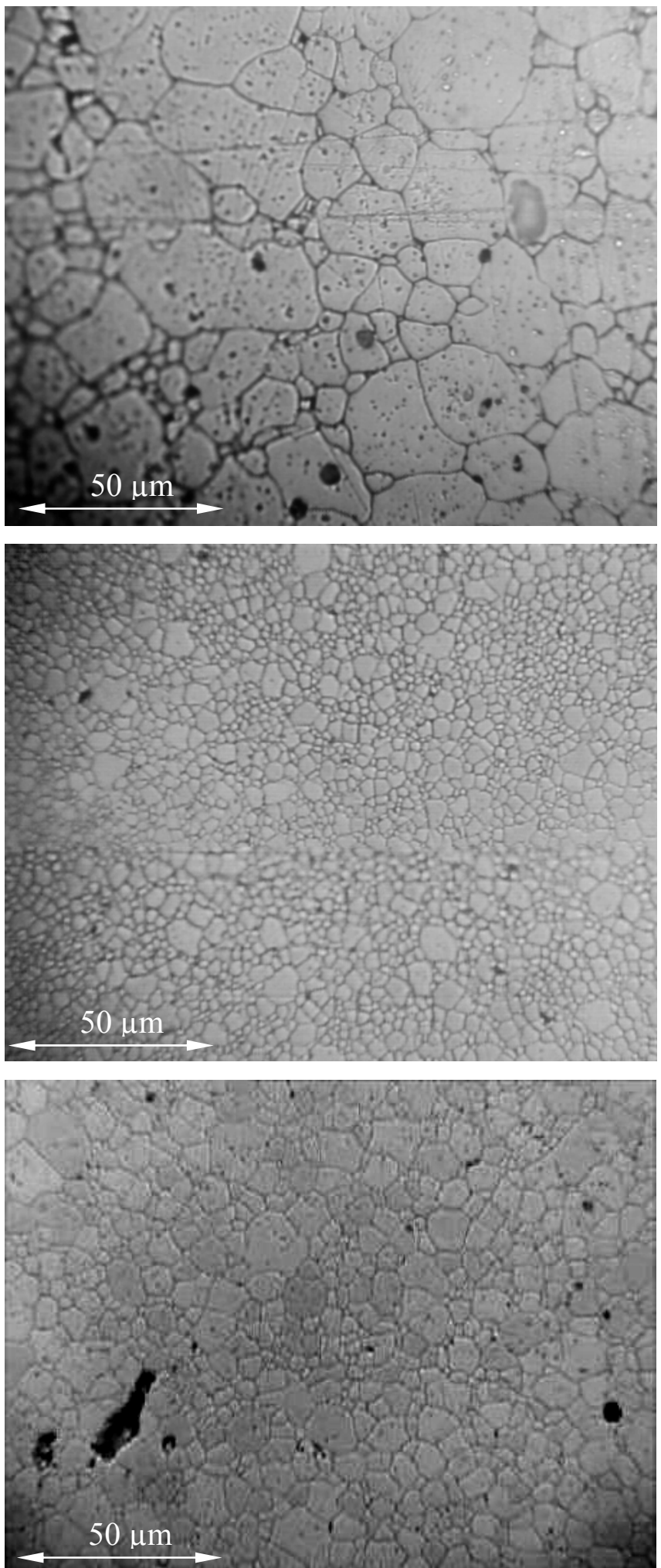

a) konventionelle Widerstandsbeheizung,

4-7 K/min Aufheizrate,

$500 \mathrm{~min}$ isotherme Haltezeit.

b) Mikrowellen-Widerstands-

Hybridbeheizung,

$25 \mathrm{~K} / \mathrm{min}$ Aufheizrate,

$30 \mathrm{~min}$ isotherme Haltezeit.

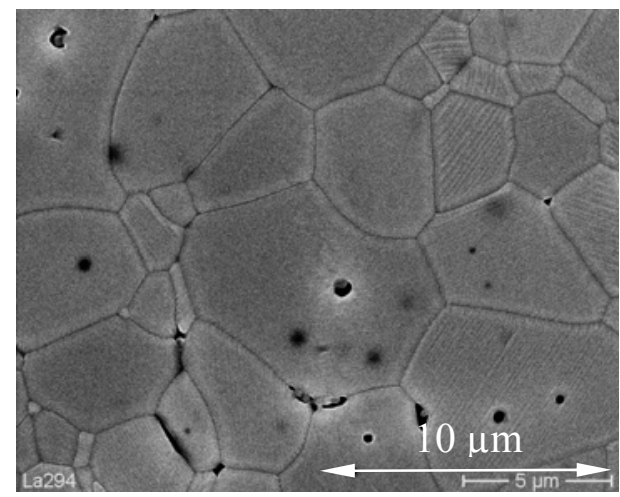

c) Schnelle Widerstandsbeheizung, $25 \mathrm{~K} / \mathrm{min}$ Aufheizrate, $30 \mathrm{~min}$ isotherme Haltezeit.

Abbildung 4.24 Gefügestruktur von konventionell und mit Mikrowelle gesintertem NiZn-Ferrit [95].

Die unterschiedlichen Sinterbedingungen zeigen sich auch in den unterschiedlichen Gefügen der Proben (Abbildung 4.24). Das langsame Aufheizen und die lange Sinterzeit beim konventionellen Sintern führen zu einem grobkörnigen, bimodalen Gefüge mit hoher intragranularer Porosität und Korngrößen von 10 - $50 \mu \mathrm{m}$. Offensichtlich stellen 
die Poren auch hier kein wesentliches Hindernis für die sich bewegenden Korngrenzen dar. In starkem Gegensatz hierzu besitzt die mit Mikrowellen gesinterte Probe b) ein sehr feinkörniges, relativ porenfreies Gefüge mit Korngrößen kleiner $10 \mu \mathrm{m}$. Die auftretenden Poren sind sehr fein und liegen sowohl intra- als auch intergranular vor. Etwas grobkristalliner und mit erhöhter Porosität ist das Gefüge bei Probe c).

\subsubsection{Optimierung des Mikrowellenanteils bei NiZn-Ferriten}

In einer weiteren Versuchsreihe wurde der Mikrowellenanteil optimiert. Allerdings lässt sich konstruktionsbedingt die Leistung der Magnetrons und damit der Mikrowellenanteil nicht beliebig absenken. Bei eingeschalteter Mikrowellenheizung ist ein Anteil von mindestens $25 \%$ Mikrowellenenergie vorhanden. Die sonstigen Prozessparameter entsprechen Tabelle 4.7 b). Untersucht wurden die Permeabilität, die Dichte und die Korngröße der Proben (s. Abbildung 4.25, Abbildung 4.26 und Abbildung 4.27).

Bei ungefähr 30\%-35\% Mikrowellenanteil zeigt die Anfangspermeabilität ein Maximum bei Werten größer 1800.

Mit der im Gegensatz zu den vorherigen Untersuchungen nochmals präzisierten Dichtemessungen erkennt man nun auch hier leichte Unterschiede. Die gemessenen Dichten sind bei den Proben mit höchster Permeabilität sehr hoch und liegen mit Werten um 5,24 g/ $\mathrm{cm}^{3}$ bei ca. $97 \%$ der theoretischen Dichte. Auffallend ist der Unterschied zur ohne Mikrowelle gesinterten Probe mit einer Dichte von nur 5,19 g/ $\mathrm{cm}^{3}$.

Anders verhält es sich mit der Korngröße. Sie ist bei den Proben mit höchster Permeabilität minimal und wächst mit zunehmendem Mikrowellenanteil an. Dies steht in direktem Gegensatz zu den in Abschnitt 2.1.7 erörterten, aus der Literatur bekannten Ergebnissen.

Die mit reiner Widerstandserwärmung gesinterte Probe hat abweichend ein relativ grobkörniges Gefüge. 


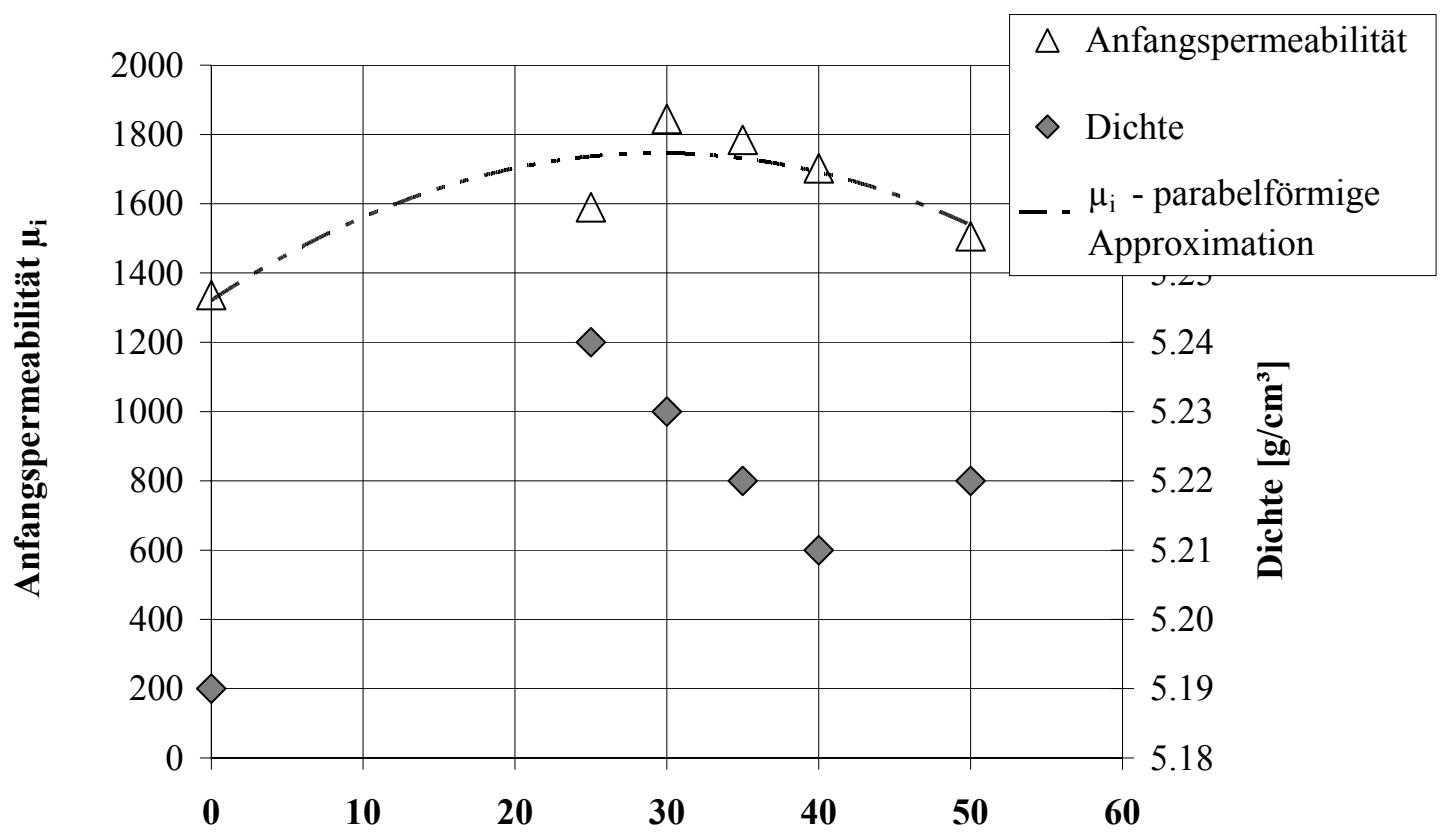

Anteil an Mikrowellenleistung [\%]

Abbildung 4.25 Permeabilität und Sinterenddichte in Abhängigkeit vom Anteil an Mikrowellenleistung für NiZn-Ferrite [95].

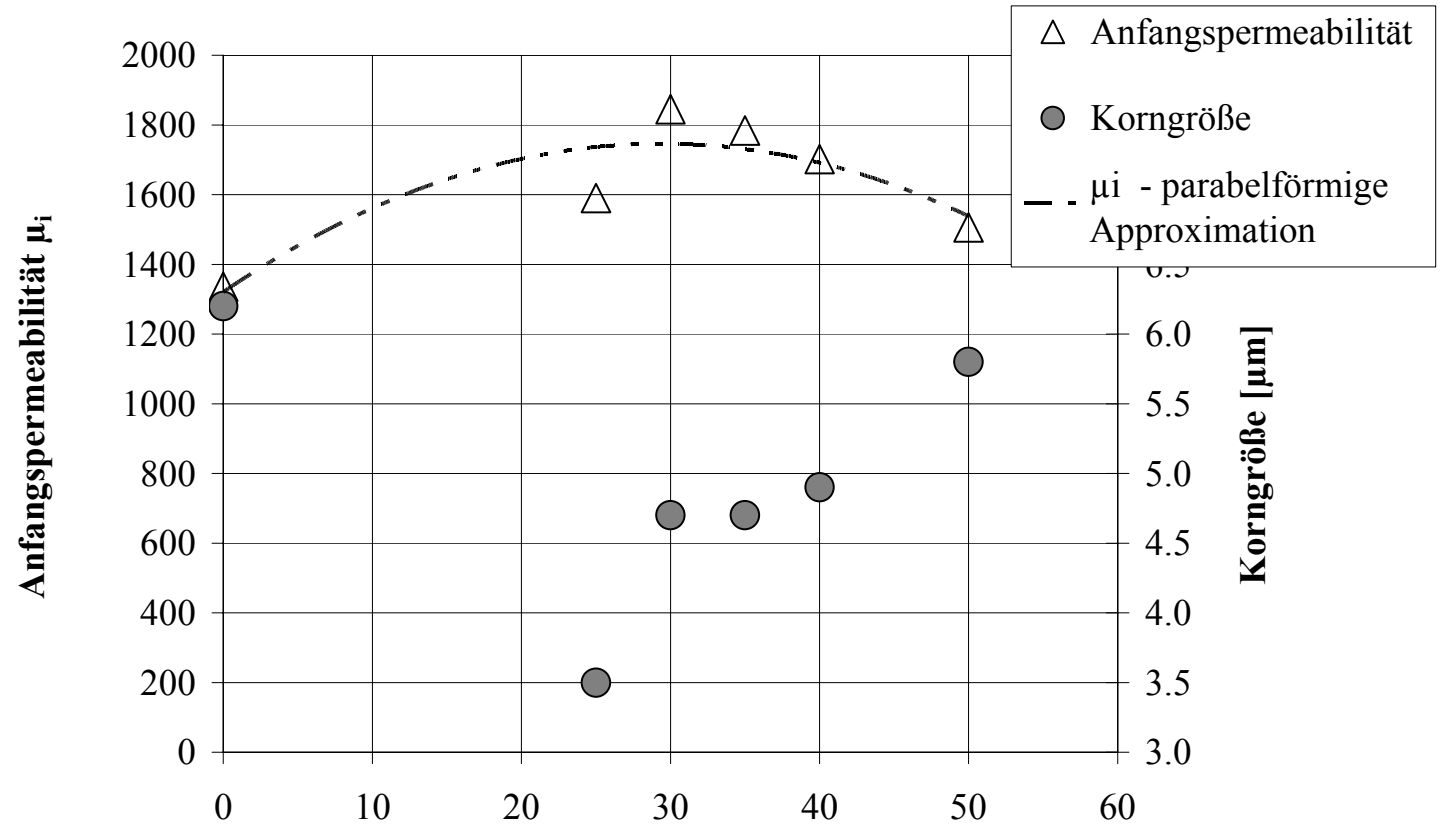

Anteil an Mikrowellenleistung [\%]

Abbildung 4.26 Permeabilität und Korngröße bei den mit Mikrowelle gesinterten NiZn-Ferriten [95]. 


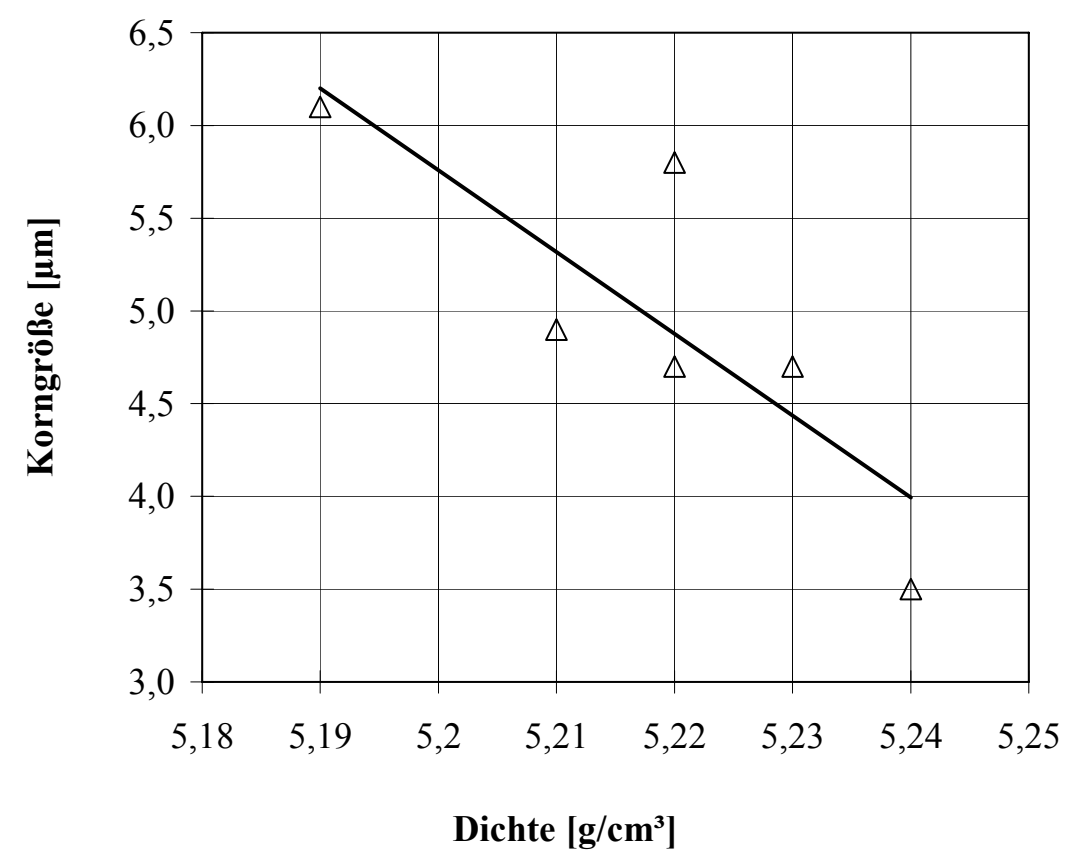

Abbildung 4.27 Zusammenhang zwischen Dichte und Korngröße. Mit zunehmender Dichte nimmt die Korngröße ab.

\subsubsection{Versuche mit MnZn-Ferriten für die Leistungsübertragung}

Untersucht wurden zwei unterschiedliche ringförmige Proben, mit den Abmessungen $\varnothing_{\text {außen }} \times \varnothing_{\text {innen }} \mathrm{x} \mathrm{h} \approx 16 \mathrm{~mm}$ x $10 \mathrm{~mm}$ x $5 \mathrm{~mm}$, aus dem Material L1 (vgl. Abschnitt 3.1.2). Die Sinterung erfolgte zum einen konventionell, mit Widerstandsbeheizung und niedrigen Aufheizraten, zum anderen wieder mit kombinierter Widerstands-MikrowellenHybridbeheizung mit einem Mikrowellenanteil von $30 \%$ und Aufheizraten von $25 \mathrm{~K} / \mathrm{min}$. Um die kurze isotherme Haltezeit bei der Hybridsinterung zu kompensieren wurde die Temperatur von $1160{ }^{\circ} \mathrm{C}$ auf $1200^{\circ} \mathrm{C}$ angehoben. Eine Übersicht über die Versuchsbedingungen und Resultate gibt Tabelle 4.8. Das wichtigste Ergebnis ist die Absenkung der Ummagnetisierungsverluste bei einer um $60 \%$ verkürzten Sinterzeit. Ursache ist das feinere Gefüge der hybridgesinterten Probe, das zu einer merklichen Abnahme der Wirbelstromverluste führt. Nimmt man das Vorherrschen intrakristalliner Wirbelströme an, so berechnet sich nach Gleichung 2.15 eine Verringerung des Wirbelstromverlustfaktors $\alpha_{w}$ um $31 \%$, bei Abnahme der mittleren Korngröße von 4,6 $\mu$ m auf 3,7 $\mu \mathrm{m}$. Die Verlustauftrennung nach Gleichung 2.16 ergibt in guter Übereinstimmung eine Abnahme um 33 \% und bestätigt damit die Dominanz intrakristalliner Wirbelströme. Allerdings nimmt aufgrund des feineren Gefüges der Hystereseverlustfaktor $\alpha_{\mathrm{h}}$ um $31 \%$ zu, was jedoch wegen der linearen Frequenzabhängigkeit der Hystereseverluste bei höheren Frequenzen eine untergeordnete Rolle spielt. Wie die Verlustauftrennung in 
Abbildung 4.29 erkennen lässt, sind die Ummagnetisierungsverluste der hybridgesinterten Probe schon bei Frequenzen größer 300 kHz kleiner als bei den konventionell gesinterten Proben.

\begin{tabular}{l|c|c}
\hline Eigenschaft & $\begin{array}{l}\text { a) Widerstandsbeheizung, } \\
\text { niedrige Heizrate }\end{array}$ & $\begin{array}{l}\text { b) Elektro-Mikrowellen- } \\
\text { Hybridsinterofen }\end{array}$ \\
\hline Ofenvolumen & $0,63 \mathrm{~m}^{3}(\mathrm{vgl} .3 .3 .1)$ & $0,01 \mathrm{~m}^{3}(\mathrm{vgl} .3 .3 .2)$ \\
\hline Heizung & Widerstandsbeheizung & $\begin{array}{c}\text { Widerstands - MW } \\
\text {-Beheizung } 30 \% \mathrm{MW}\end{array}$ \\
\hline Aufheizrate & $5 \mathrm{~K} / \mathrm{min}$ & $25 \mathrm{~K} / \mathrm{min}$ \\
\hline Isotherme Haltezeit & $300 \mathrm{~min}$ & $30 \mathrm{~min}$ \\
\hline Isotherme Sintertemperatur & $1160^{\circ} \mathrm{C}$ & $1200{ }^{\circ} \mathrm{C}$ \\
\hline $\begin{array}{l}\text { Länge des Ofenzyklus } \\
\text { kalt-kalt) }\end{array}$ & $900 \mathrm{~min}$ & $200 \mathrm{~min}$ \\
\hline Dichte der Ferritproben & $4,5 \mathrm{~g} / \mathrm{cm}^{3}$ & $4,5 \mathrm{~g} / \mathrm{cm}^{3}$ \\
\hline $\begin{array}{l}\text { mittlere Korngröße } \bar{X} \\
\text { Anfangspermeabilität } \mu_{i} \text { der } \\
\text { Proben bei } 10 \mathrm{kHz}\end{array}$ & $4,6 \mu \mathrm{m}$ & $3,7 \mu \mathrm{m}$ \\
\hline $\begin{array}{l}\text { Ummagnetisierungsverluste } \\
\text { bei } 100{ }^{\circ} \mathrm{C}, 50 \mathrm{mT}, 1 \mathrm{MHz}\end{array}$ & 1200 & 1000 \\
\hline $\begin{array}{l}\text { Wirbelstromverlustfaktor } \\
\alpha_{\mathrm{w}}\end{array}$ & $620 \mathrm{~W} / \mathrm{m}^{3}$ & $440 \mathrm{~W} / \mathrm{m}^{3}$ \\
\hline $\begin{array}{l}\text { Hystereseverlustfaktor } \\
\alpha_{\mathrm{h}}\end{array}$ & $5,410^{-4} \mathrm{Ws} / \mathrm{m}^{3}$ & $3,610^{-4} \mathrm{Ws} / \mathrm{m}^{3}$ \\
\hline
\end{tabular}

Tabelle 4.8 Vergleich zweier unterschiedlicher Sinterzyklen für MnZn-Leistungsferrite.

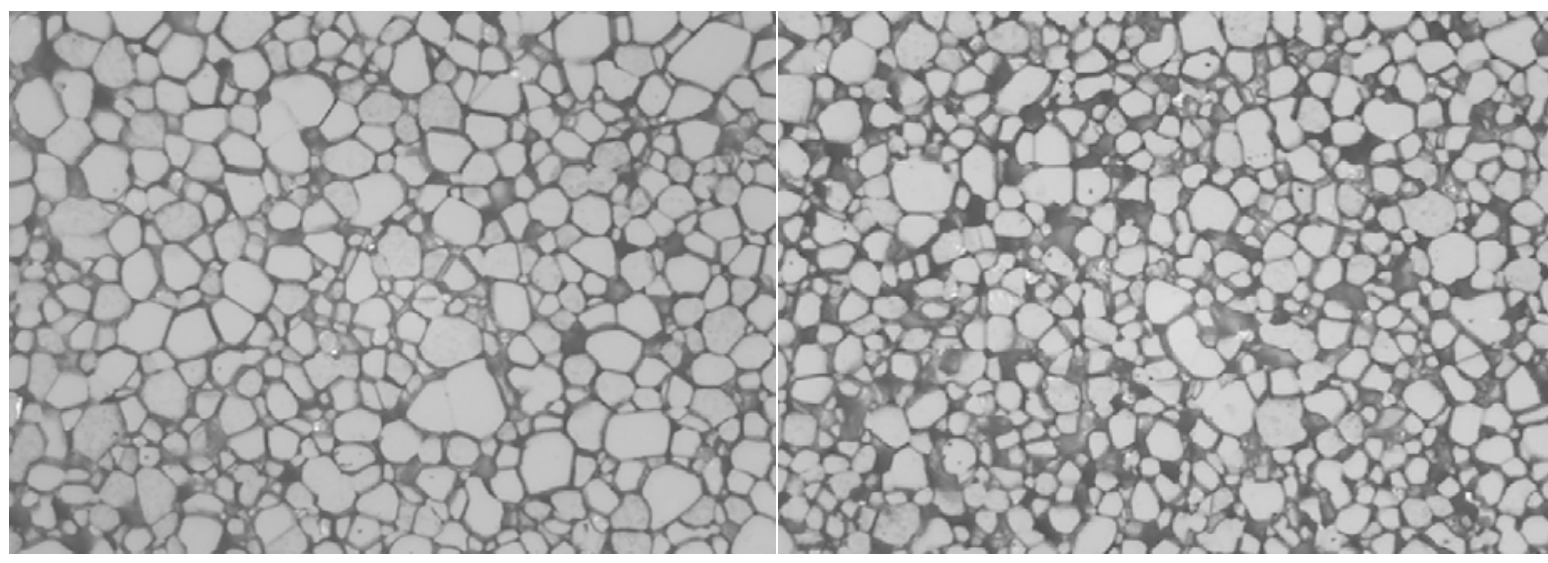

a)

Abbildung 4.28
$20 \mu \mathrm{m}$

b)

Gefügeaufnahmen einer konventionell gesinterten Probe a) im Vergleich zum mit einem Mikrowellenanteil von $30 \%$ gesinterten Ferrit b) [84]. 
a) konventionell gesintert

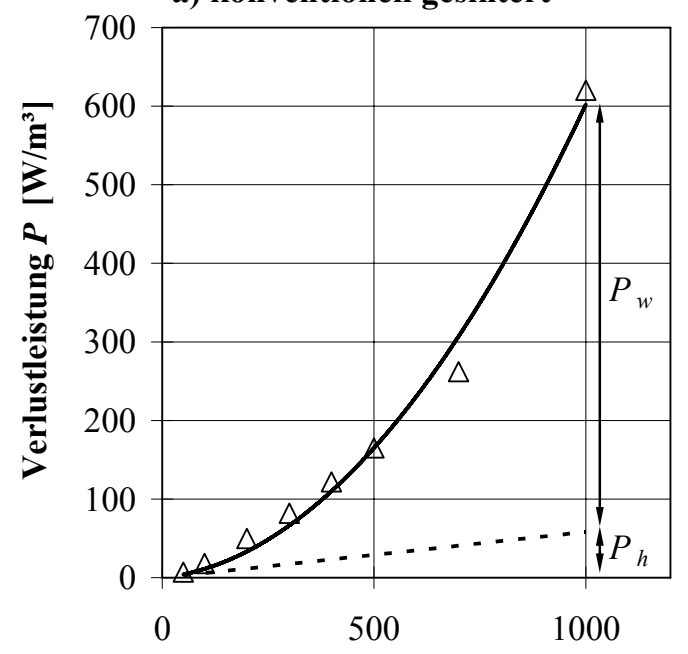

Frequenz $f[\mathrm{kHz}]$ b) Mikrowelle hybridgesintert

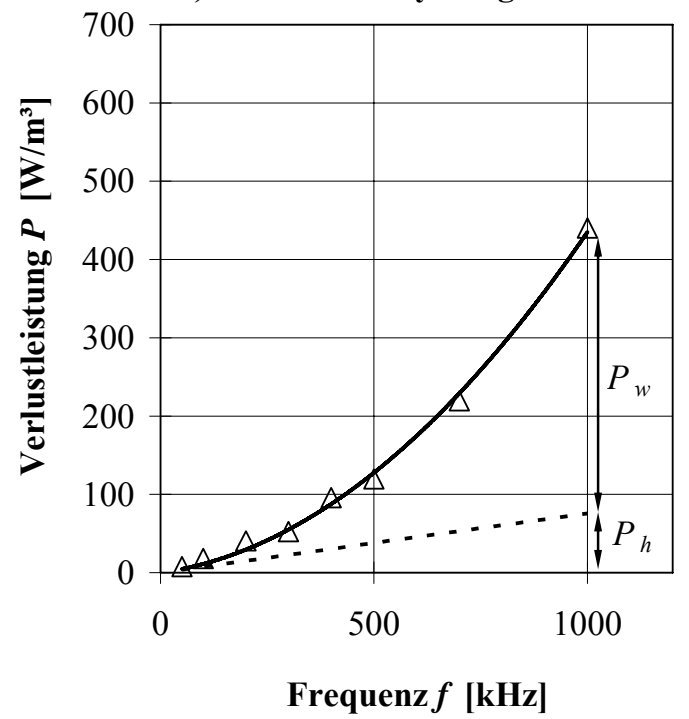

Abbildung 4.29 Auftrennung der Ummagnetisierungsverluste in Hystereseverluste und Wirbelstromverluste gemäß Gleichung 2.16 für die konventionell gesinterte Probe a) im Vergleich zum mit Mikrowelle hybridgesinterten Ferrit b). Gemessen bei $B=\mathbf{5 0}$ mT und $T=100{ }^{\circ} \mathrm{C}$.

\subsubsection{TEM-Untersuchung der Korngrenzen}

Die TEM-Untersuchungen ${ }^{[i]}$ an den hybridgesinterten Proben belegen das schon bei den Lichtmikroskopischen Untersuchungen aufgefallene dichte feinkristalline Gefüge.

Abbildung 4.30 zeigt die porösere, durch die Probenpräparation teilweise ausgebrochene, Mikrostruktur der MnZn-Ferritprobe. Typische Korngrößen liegen im Bereich weniger Mikrometer, zum Teil aber auch darunter. Die untersuchten Korngrenzen und Tripelpunkte sind frei von Poren (Abbildung 4.32).

Allerdings zeigen sich sowohl bei den MnZn-Ferriten als auch bei den NiZn-Ferriten vereinzelte Kornzwischenphasen mit einer Breite von wenigen nm. Größere in den Tripelpunkten liegende Ausscheidungen, wie sie teilweise in der Literatur beschrieben werden [96-98] wurden nicht gefunden (Abbildung 4.32, Abbildung 4.33 und Abbildung 4.31).

[i] Die TEM-Aufnahmen in diesem Abschnitt wurden im Rahmen des vom BMBF geförderten Projektes Einsatz von nanodispersen Pulvern zur Herstellung von Mn-Ferriten mit verbesserten Eigenschaften vom MPI für Mikrostrukturanalyse in Halle erstellt. 


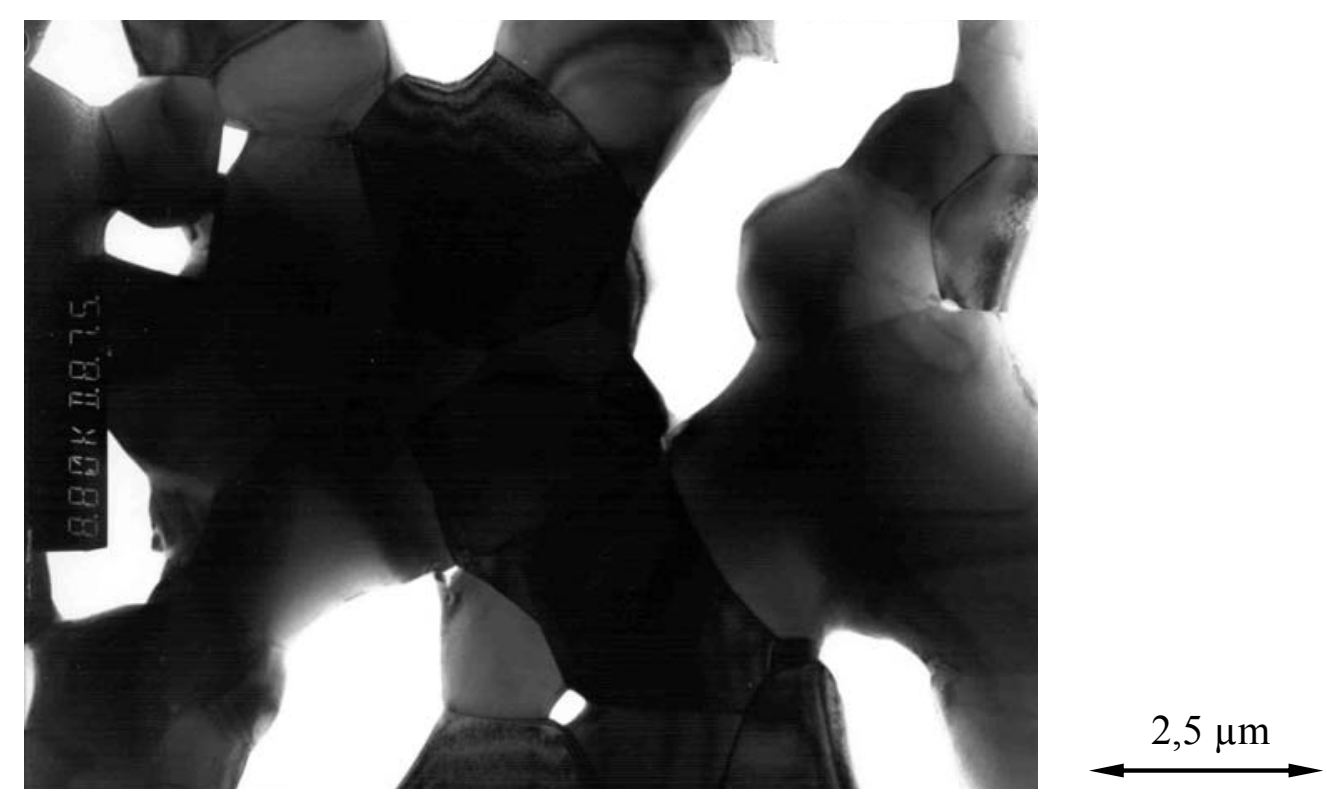

Abbildung 4.30 TEM-Aufnahme einer mit 30\% Mikrowellenleistung gesinterten MnZnFerritprobe (Tabelle 4.8 b). Bei den Hohlräuumen handelt es sich um Poren oder um bei der Präparation ausgebrochene Körner. Dazwischen ist ein sehr feines und dichtes Gefüge erkennbar.

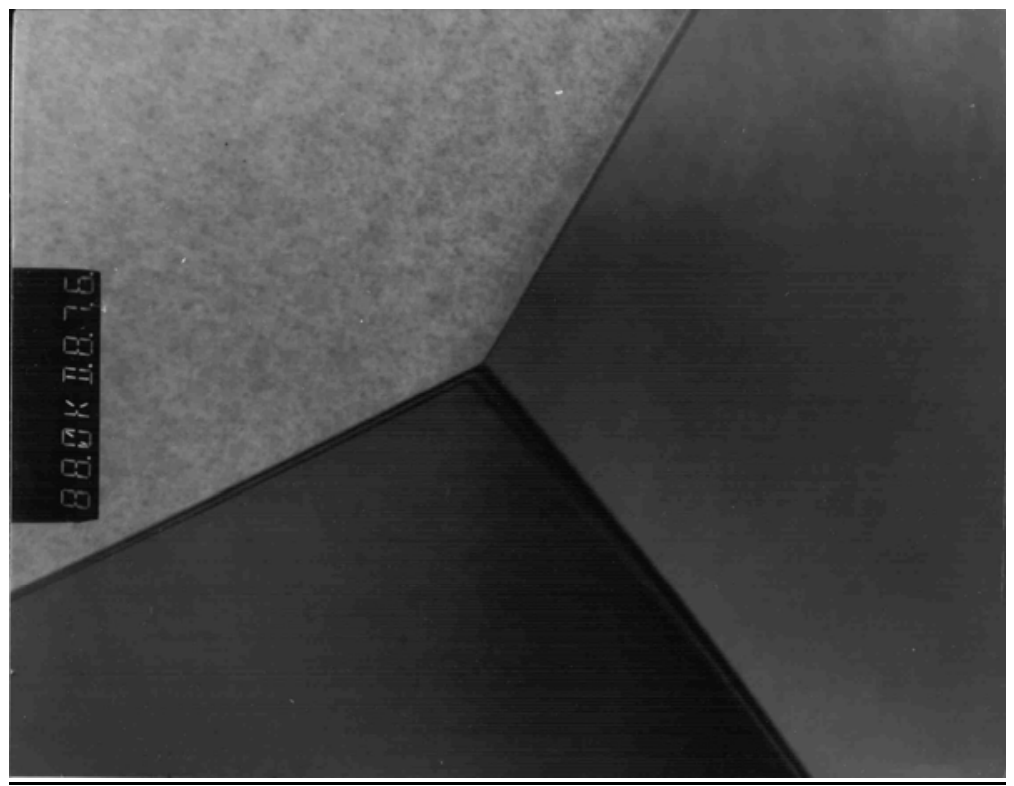

$200 \mathrm{~nm}$

Abbildung 4.31 Ferritkernprobe wie in Abbildung 4.30. Auch bei erhöhter Vergrößerung sind Tripelpunkt, Korngrenzen und Körner weitestgehend frei von Poren. 


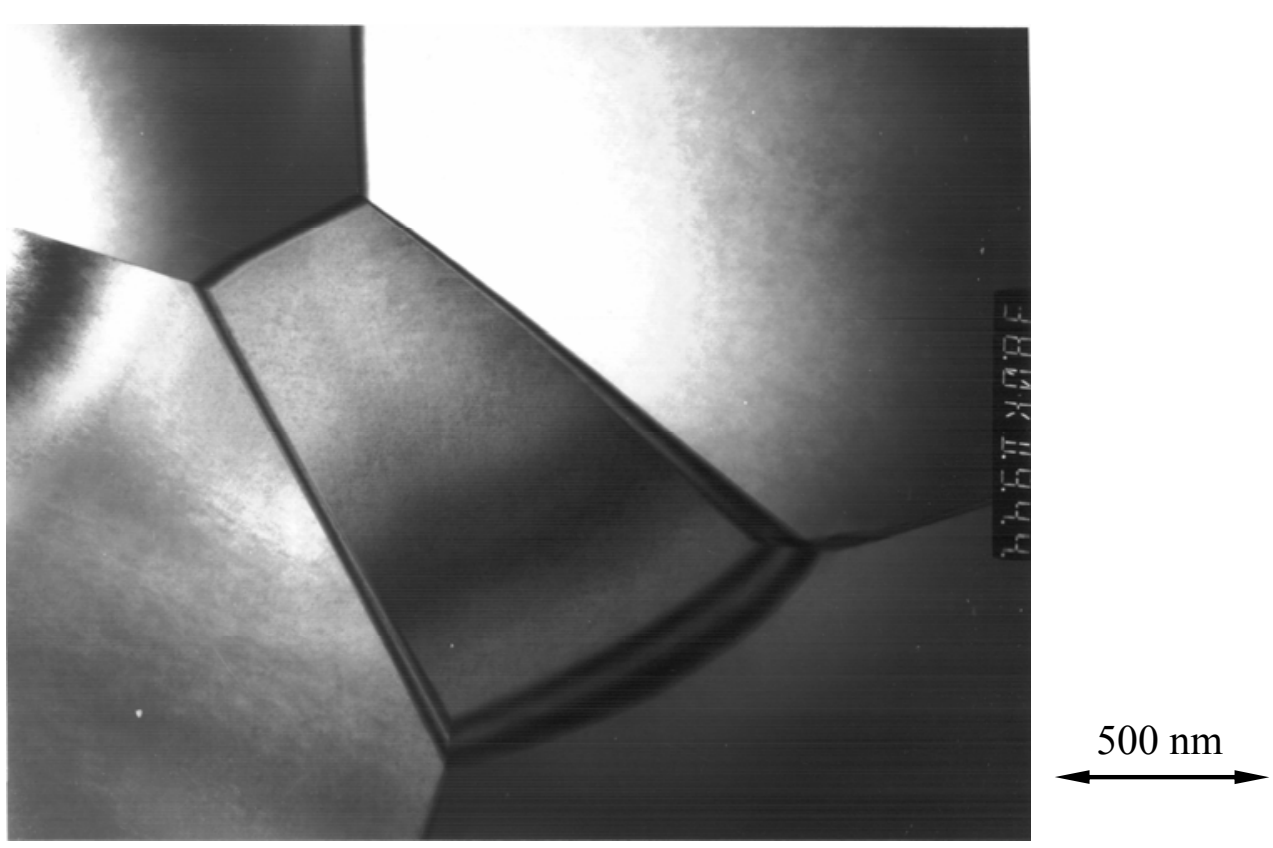

Abbildung 4.32 TEM-Untersuchung an einer mit 30\% Mikrowellenleistung hybridgesinterten NiZn-Ferritprobe. In Korngrenzen und Tripelpunkten ließen sich keine Poren nachweisen.

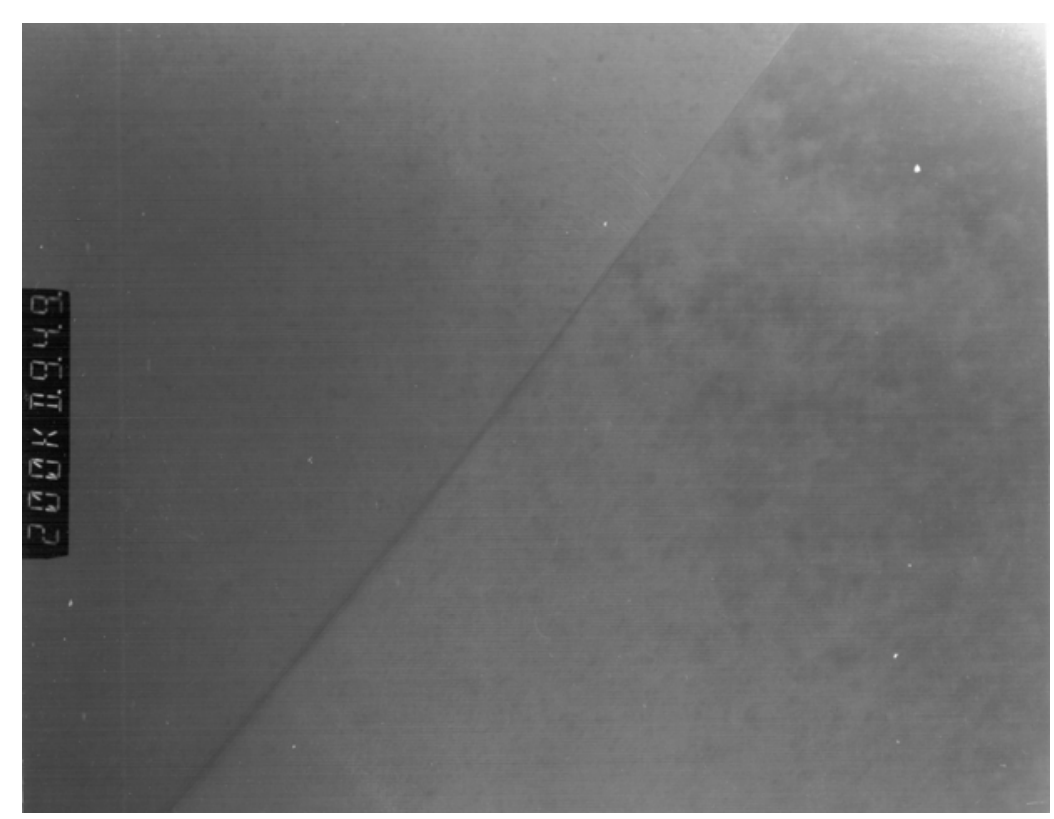

$100 \mathrm{~nm}$

Abbildung 4.33 Kornzwischenphase mit einer Breite von ca. $2 \mathrm{~nm}$ finden sich bei der Probe aus Abbildung 4.32. 


\section{Diskussion}

\subsection{Wirkung von $\mathrm{Bi}_{2} \mathrm{O}_{3}$ und $\mathrm{MoO}_{3}$ als schmelzfließende Zusätze}

\subsubsection{Charakterisierung der Phasen}

Wie die Untersuchungen zur Wismutoxid-Ferrit-Phasengrenze in Abschnitt 4.1.1 zeigen, bildet Wismutoxid mit den anderen im Ferritpressling vorliegenden Metalloxiden bzw. Mischoxiden eutektische Systeme. Es kommt zum Schmelzfluss bei Temperaturen von $700-800^{\circ} \mathrm{C}$. Die mikroskopischen Untersuchungen zeigen hierbei das Entstehen zweier übereinanderliegender, flüssiger Schichten von geringer Mischbarkeit. In der aus ca. 98 Mol.\% Wismutoxid bestehenden Deckschicht ließen sich Ausscheidungen mit einer ungefähren Zusammensetzung von

$$
\begin{gathered}
53 \mathrm{Mol} . \% \mathrm{Bi}_{2} \mathrm{O}_{3}, \\
31 \mathrm{Mol} \% \mathrm{Fe}_{2} \mathrm{O}_{3}, \\
14 \mathrm{Mol} \% \mathrm{MnO}, \\
2 \mathrm{Mol} \% \mathrm{ZnO},
\end{gathered}
$$

nachweisen. Die darunterliegende zweite Schicht hat eine ungefähre Zusammensetzung von

$$
\begin{gathered}
45 \mathrm{Mol} \% \mathrm{Bi}_{2} \mathrm{O}_{3}, \\
31 \mathrm{Mol} \% \mathrm{Fe}_{2} \mathrm{O}_{3}, \\
17 \mathrm{Mol} \% \mathrm{MnO}, \\
7 \mathrm{Mol} \% \mathrm{ZnO} .
\end{gathered}
$$

Dieses Verhalten unterscheidet sich von den von Viting und Golubkova an Co- und NiFerriten durchgeführten Untersuchungen [69], in denen jeweils nur ein einziges Eutektikum bei einer eutektischen Konzentration von 13,5 Mol.\% bzw. 9 Mol.\% Ferrit nachgewiesen wurde (Abbildung 2.12).

Ein Ansatz zur Erklärung der Unterschiede ist das bei den MnZn-Ferriten nach der Kalzination vorhandene, oxidische Phasengemisch. Rossel [79] weist das Auftreten von $\mathrm{Fe}_{2} \mathrm{O}_{3}$ (Hämatit), $\mathrm{MnFe}_{2} \mathrm{O}_{4}$ (Jacobsit) und $\mathrm{Mn}_{2} \mathrm{O}_{3}$ (Bixbyit - C) nach. Zusätzlich entsteht beim Erwärmen des Formkörpers ab $600{ }^{\circ} \mathrm{C}$ Zn-Ferrit $\left(\mathrm{ZnFe}_{2} \mathrm{O}_{4}\right)$ und ab ca. $800{ }^{\circ} \mathrm{C}$ MnZn-Ferrit [78, 80] (vgl. Abschnitt 3.2.1). Es handelt sich also streng genommen nicht um ein binäres, sondern um ein Mehrkomponenten-System, das Eutektika unterschiedlicher Konzentrationen mit entsprechend auch unterschiedlichen eutektischen Temperaturen ausbildet. Wahrscheinlich exisitert bei $800^{\circ} \mathrm{C}$ bei den MnZn-Ferriten 
neben der Spinellphase insbesondere Hämatit $\left(\mathrm{Fe}_{2} \mathrm{O}_{3}\right)$. Im Gegensatz dazu sind sowohl Nickel- als auch Kobaltferrit gegen Oxidation stabile Systeme und liegen daher phasenrein als Spinell vor.

In diesem Kontext sind Untersuchungen mittels Hochtemperatur-Röntgendiffraktometrie, die von Levin und Roth an mehreren Metalloxid - Wismutoxid Systemen durchgeführt wurden [99], von Interesse. Beim Hämatit - Wismutoxid System finden die Autoren eine kubisch - raumzentrierte (krz) Phase bei einem Gehalt von ca. 4 Mol.\% $\mathrm{Fe}_{2} \mathrm{O}_{3}$ und vermuten eine weitere, metastabile $\mathrm{BiFeO}_{3}$ Phase, die ein zusätzliches Eutektikum bildet (Abbildung 5.1).

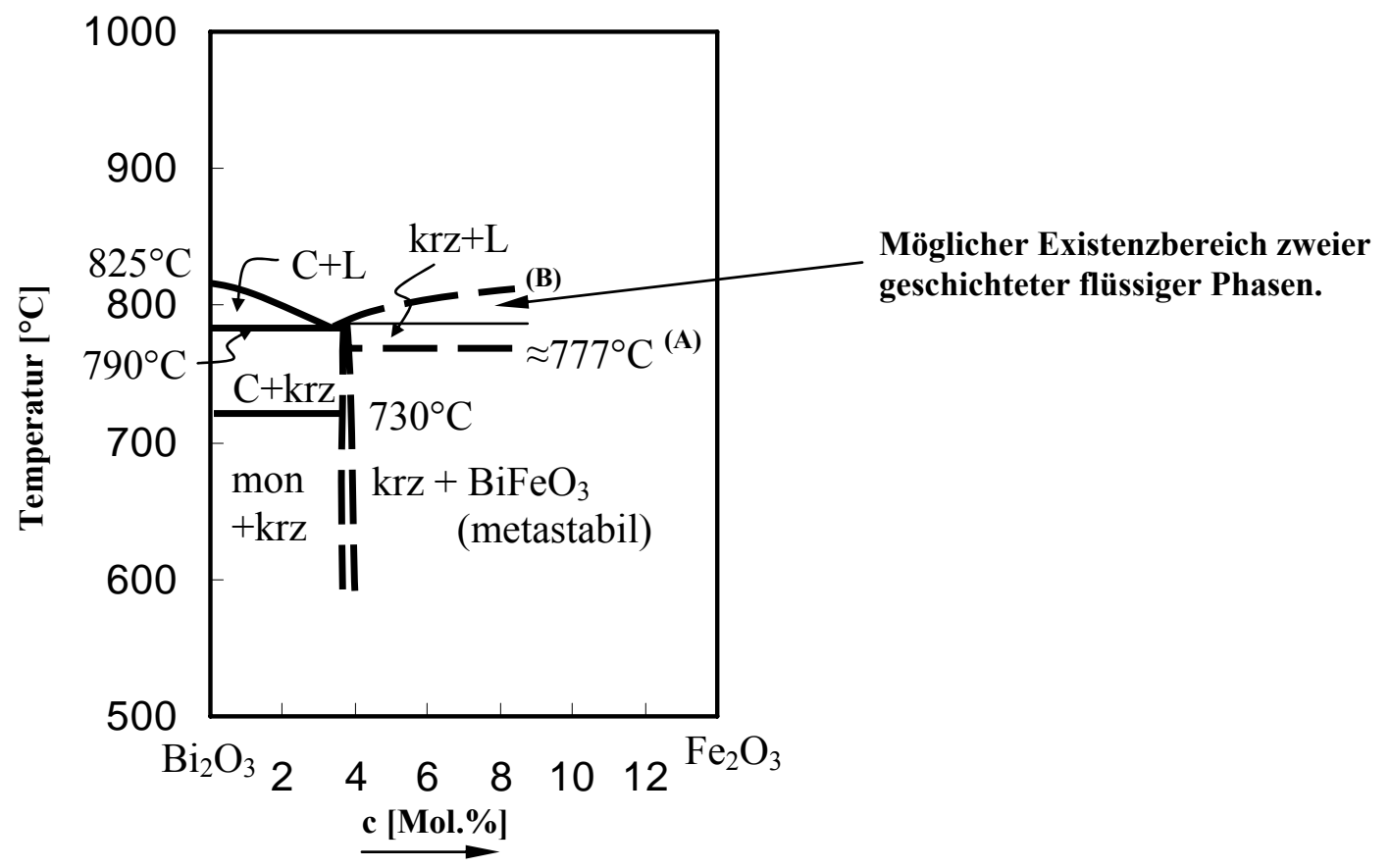

Abbildung 5.1 Phasendiagramm des Systems $\mathrm{Bi}_{2} \mathrm{O}_{3}-\mathrm{Fe}_{2} \mathrm{O}_{3}$, gemessen mittels Hochtemperatur Röntgendiffraktometrie nach [99]. Neben dem Eutektikum bei ca. 3,8 Mol.\% $\mathrm{Bi}_{2} \mathrm{O}_{3}$ existiert eine stabile krz-Phase mit einem Gehalt von ca. $4 \mathrm{Mol} . \% \mathrm{Fe}_{2} \mathrm{O}_{3}$. Vermutet wird weiterhin ein metastabiles Eutektikum zwischen der krz - Phase und der metastabilen $\mathrm{BiFeO}_{3}$-Phase. Die eutektische Temperatur (A) liegt bei ca. $777^{\circ} \mathrm{C}$. Das Maximum der Liquidus-Kurve (B) liegt über dem Schmelzpunkt der krz-Phase.

Im Zusammenhang mit diesem zweiten Eutektikum lassen sich sowohl die gemessenen hohen Konzentrationen an $\mathrm{Fe}_{2} \mathrm{O}_{3}$ in der Zwischenschicht und in den Ausscheidungen, als auch das Auftreten zweier geschichteter flüssiger Phasen verstehen. Es handelt sich wahrscheinlich um chemisch mit $\mathrm{BiFeO}_{3}$ verwandte Phasen oder Phasengemische mit einem entsprechenden Anteil an Mn- und Zn-Ionen. Insbesondere die Substitution von $\mathrm{Fe}^{3+}$ durch $\mathrm{Mn}^{3+}$ ist aufgrund der gleichen Wertigkeiten und wegen der ähnlichen Ionenradien (0,64 Á bzw. 0,66 ̊́ [93]) schlüssig. 
Die durchgeführten Untersuchungen bestätigen weiterhin die gut benetzenden Eigenschaften und die daraus resultierende schnelle Infiltration des offenporigen Ferrits und damit eine merkliche Verringerung der Oberflächenenergie im benetzten Zustand.

\subsubsection{Zugabe kleinster Mengen}

Bei Zugabe von Mengen von ca. 0,02 - 0,03 Gew.\% Wismutoxid wird die Korngrenzenbeweglichkeit erhöht und das Kornwachstum beschleunigt. Die mittlere Korngröße nimmt auf ca. 25 - $30 \mu \mathrm{m}$ zu. Dies drückt sich in einer Abnahme der Aktivierungsenergie um ca. $0,1-0,15 \mathrm{eV}$ in der das Kornwachstum beschreibenden Gleichung 2.23 aus. Eine Änderung des Exponenten $n$ konnte nicht nachgewiesen werden. Sowohl bei den dotierten als auch bei den undotierten Proben ergab sich in guter Übereinstimmung mit der vorhandenen Literatur $n \approx 3[50,51]$.

Der Kapillardruck als Triebkraft für die Verdichtung nimmt bei Abnahme der Korngrenzenenergie (vgl. Gleichung 2.18) zu. Wahrscheinlich verbessert sich gleichzeitig die Diffusion in den Korngrenzen, was in der Summe zu der beobachteten Abnahme der Porosität von über 2,5\% auf unter 1,5\% führt.

Es kommt im Idealfall noch nicht zur Entstehung intrakristalliner Poren und zu diskontinuierlich gewachsenen Körnern, sondern Poren können sich durch Diffusion entlang der Korngrenzen abbauen (vgl. Abbildung 4.10). Die Anfangspermeabilität $\mu_{i}$ nimmt proportional zur Korngröße von 10000 auf ca. 14000 zu.

Anders verhalten sich die mit Molybdäntrioxid dotierten Proben. Hier beobachtet man schon bei Zugabe von ca. 0,02 Gew. $\% \mathrm{MoO}_{3}$ eine Erhöhung der intrakristallinen Porosität, das Losreißen von Korngrenzen aufgrund einer im Vergleich zum $\mathrm{Bi}_{2} \mathrm{O}_{3}$ erhöhten Korngrenzenmobilität und folglich ein Anwachsen der mittleren Korngröße von $20 \mu \mathrm{m}$ auf $45 \mu \mathrm{m}$. Die Anfangspermeabilität $\mu_{i}$ erreicht sehr hohe Werte von ca. 17000. Diese Ergebnisse passen gut $\mathrm{zu}$ den von Matsuo et al. [73] durchgeführten Untersuchungen (vgl. Abschnitt 2.2.4).

\section{$\underline{\text { TEM - Untersuchungen }}$}

Die TEM-Untersuchungen an den wismutoxiddotierten Proben belegen das Auftreten intrakristalliner Phasen bei ca. 1/3 der Korngrenzen. Der Großteil dieser Kornzwischenphasen ist während des Sinterns verflüssigt. Die Breite der flüssigen Phase variiert ungefähr von $50 \mathrm{~nm}$ bis $200 \mathrm{~nm}$. In diesen Zwischenphasen werden teilweise starke Anreicherungen von Kalzium- und Siliziumoxid gefunden. An einer Stelle ließen sich reine Siliziumoxid-Ausscheidungen nachweisen. 
Das Auftreten der flüssigen Phase bei weniger als 1/3 der Korngrenzen kann durch den sehr geringen Gehalt an Wismutoxid erklärt werden. Allerdings ist es sehr wahrscheinlich, dass ein Teil der vorkommenden Korngrenzen, insbesondere Zwillingskorngrenzen und Korngrenzen hoher Symmetrie, so niederenergetisch sind, dass sich ihre Korngrenzenenergie durch Eindringen der flüssigen Phase nicht weiter reduzieren lässt. Vergleichbare Ergebnisse wurden bisher nicht veröffentlicht.

\section{Entspannungs- und Reinigungseffekte}

Neben einem verbesserten Kornwachstum und der bei den mit $\mathrm{Bi}_{2} \mathrm{O}_{3}$ dotierten Proben beobachteten verbesserten Verdichtung, gibt es einen Entspannungs- und Reinigungseffekt. Fremdatome, die sich ohne Zugabe der schmelzenden Additive teilweise im Spinell lösen und $\mathrm{zu}$ Gitterspannungen führen, reichern sich in den Kornzwischenphasen an. Bei den undotierten Proben führen hingegen lokale, im Kristallgitter vorhandene Spannungen zu einer Zunahme der magnetoelastischen Energie und damit verbunden $\mathrm{zu}$ einer Verschlechterung der magnetischen Eigenschaften (vgl. Abschnitt 2.1.3). Insbesondere nimmt nach Gleichung 2.9 die Anfangspermeabilität ab. Interessant sind hierbei die Untersuchungen von Nakata et al., die eine Verschlechterung der magnetischen Eigenschaften bei hochpermeablen MnZn-Ferriten aufgrund von Gitterdefekten in der Nähe der Korngrenze direkt nachweisen [97]. Daneben wird der Abbau von Gitterspannungen durch die in der Schmelze nachgewiesenen hohen Konzentrationen an Kalzium und Silizium und durch folgende Argumente gestützt.

Zum einen ist dies das in Abbildung 4.12 e) gezeigte Gefüge der mit 0,04 Gew.\% $\mathrm{MoO}_{3}$ dotierten Probe, welches aufgrund der geringen Korngröße und der hohen Porosität eine relativ niedrige Anfangspermeabilität erwarten lässt (vgl. Abschnitt 2.1 .7 und [29]). Gemessen wurden jedoch verhältnismäßig hohe Permeabilitäten $>15000$. Ähnliches findet man für Wismutoxiddotierung auch bei Drofenik et al. [70] (vgl. Abschnitt 2.2.4), wo bis $\mathrm{zu}$ einer Konzentration von 0,05 Gew.\% $\mathrm{Bi}_{2} \mathrm{O}_{3}$ keine Verstärkung des Kornwachstums, jedoch eine Erhöhung der Permeabilität von 9000 auf 11500 festgestellt wird. Eine Erhöhung der Anfangspermeabilität kann zwar, wie Abbildung 4.22 oder die Untersuchungen von Röss und Hanke [29], in Übereinstimmung mit dem Modell von Globus [27] zeigen, mit einer Zunahme der mittleren Korngröße einhergehen, tut dies jedoch nicht immer.

Zum anderen unterstützen Messungen aus früheren, an hochpermeablen MnZn-Ferriten durchgeführten Versuchen [16] die Existenz eines Entspannungseffektes. Betrachtet man nämlich den Temperaturverlauf der Anfangspermeabilität in der Nähe des sekundären 
Permeabilitätsmaximums (vgl. Abschnitt 2.1.4) bei einem Ferrit, dessen Oberfläche abgeätzt wurde und vergleicht diesen Verlauf mit dem des unbehandelten Materials, so findet sich eine Verschiebung des SPMs bei gleichzeitiger Erhöhung der Permeabilität. Dieses Verhalten wird normalerweise durch von der Oberfläche ausgehende mechanische Verspannungen erklärt, die sich durch das Abätzen lösen. Wie Abbildung 5.2 zeigt, tritt ein vergleichbarer Effekt bei Zugabe geringer Mengen von Molybdäntrioxid auf.

a)

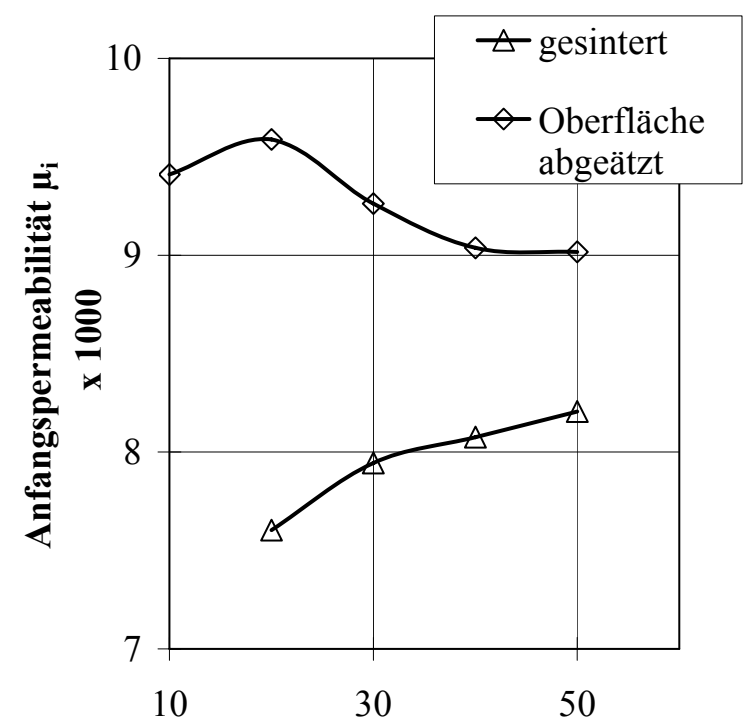

Temperatur $\mathbf{T}\left[{ }^{\circ} \mathrm{C}\right]$ b)

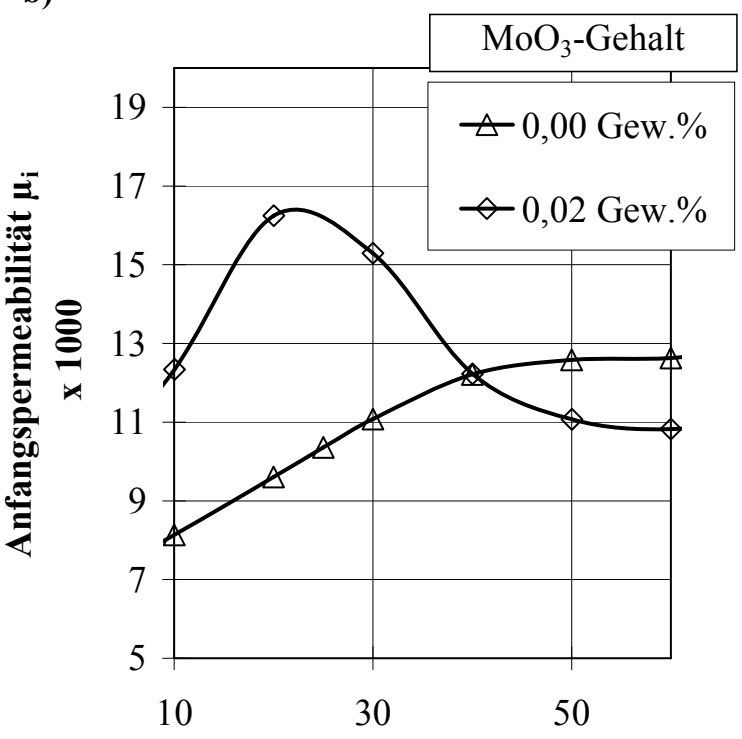

Temperatur $\mathbf{T}\left[{ }^{\circ} \mathrm{C}\right]$

Abbildung 5.2 Änderung des sekundären Permeabilitätsmaximums durch die Verringerung innerer Spannungen beim Abätzen a) und durch Zugabe geringer Mengen $\mathrm{MoO}_{3}$ b). Die in Teilabbildung a) gezeigten Ergebnisse entstammen den älteren Untersuchungen [16]. Die Zusammensetzung, die Sinterbedingungen und der $\mu(T)-$ Verlauf weichen daher etwas von den in Teilabbildung b) gezeigten Proben HP1 beziehungsweise HP1_Mo2 ab.

Kuroda et al. [100] untersuchten Leistungsferrite und führten ein ähnliches Verhalten vor allem auf eine Veränderung des im Spinell gelösten Kalziumoxidanteils zurück ${ }^{[i]}$. Bei richtig gewählten Abkühlbedingungen weisen die Autoren eine Verschiebung des sekundären Permeabilitätsmaximums in Abhängigkeit vom $\mathrm{Ca}^{2+}$-Gehalt nach (Abbildung 5.3). Allerdings werden als Erklärung weniger Entspannungsvorgänge als vielmehr eine Verschiebung des $\mathrm{Fe}^{2+} / \mathrm{Fe}^{3+}$-Gleichgewichts angenommen. Inwieweit Verspannungen im Kristallgitter oder Reduktionsvorgänge für den Effekt verantwortlich sind, lässt sich nicht abschließend klären.

[i] $\mathrm{Ca}^{2+}$ - Ionen werden während der Hochtemperaturbehandlung auf Oktaederplätzen in den Spinell eingebaut. 


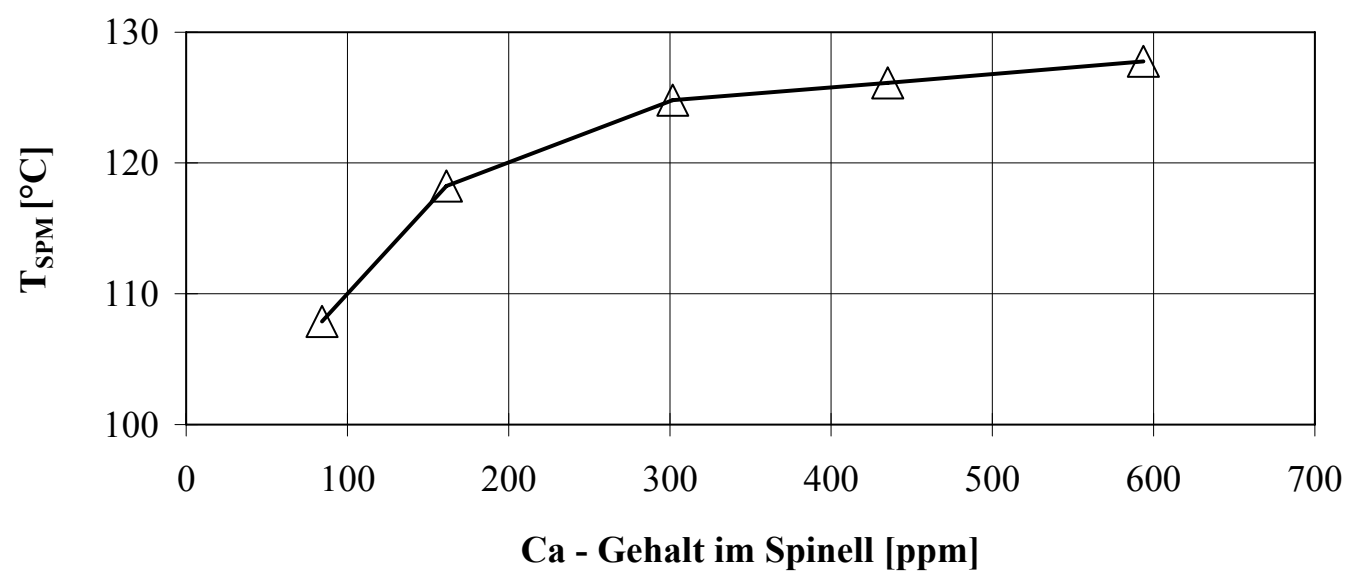

Abbildung 5.3 Verschiebung des sekundären Permeabilitätsmaximums in Abhängigkeit vom im Spinell gemessenen Ca-Gehalt für MnZn-Leistungsferrite, die nach Sinterung bei $1300{ }^{\circ} \mathrm{C}$ während des Abkühlens nochmals über 3 Stunden bei $1200{ }^{\circ} \mathrm{C}$ unter $\mathrm{N}_{2}$ Atmosphäre gehalten wurden [100].

In der Summe führen sowohl die veränderte Mikrostruktur als auch eine Verringerung von Fremdatomen im Spinellgitter zu einem Ansteigen der Anfangspermeabilität von ca. 10000 auf ca. 13500 bei den mit Wismutoxid dotierten Proben und auf ca. 17000 bei Molybdäntrioxiddotierung.

\subsubsection{Entstehung von diskontinuierlichem Kornwachstum}

Bei den mit Wismutoxid dotierten Proben kommt es bei Konzentrationen $\geq 0,04$ Gew.\% $\mathrm{zu}$ diskontinuierlichem Kornwachstum. Auffallend ist, dass sich das normalgewachsene Gefüge nur in einer Zone in der Nähe der Probenoberfläche befindet (vgl. Abbildung 4.6). Höchstwahrscheinlich verdampft ein Teil des Wismutoxids während des Sintervorgangs aus den Randzonen. Dies belegen auch die RöntgenfluoreszenzUntersuchungen aus Abschnitt 4.1.5. Die Wirkung der schmelzfließenden Phase beschränkt sich also vor allem auf das Innere des Formkörpers, wo es aufgrund einer höheren Korngrenzenmobilität zum Losreißen der Korngrenzen aus ihrer durch interkristalline Poren gegebenen Verankerung, zu diskontinuierlichem Kornwachstum und zur Ausbildung einer hohen intrakristallinen Porosität kommt.

Faktoren, die zur Erhöhung der Korngrenzenbeweglichkeit $M_{B}$ beitragen, sind insbesondere ein durch Lösung und Wiederausscheidung vereinfachter Übergang von einzelnen Ionen zwischen den benachbarten Körnern (vgl. Abschnitt 2.2 .2 und [64]) sowie die Lösung von Fremdatomen und Verunreinigungen in der flüssigen Phase. Hierzu gehören die im Gitter gelösten und über den in Abschnitt 2.2.2 beschriebenen Solute-Drag-Mechanismus wirksamen Fremdatome, wie sie im vorigen Abschnitt 
diskutiert wurden, aber auch die in der Korngrenze segregierten unlöslichen Bestandteile oder Fremdphasen.

Ein weiterer, nicht $\mathrm{zu}$ vernachlässigender Faktor ist die Verringerung der Korngrenzenenergie durch das Auftreten der flüssigen Phase. Instabile Korngrenzenkonfigurationen mit starker Krümmung werden hierdurch begünstigt (vgl. Abschnitt 2.2.2. und Abbildung 2.11). Laval et al. [101] stellen in ihrer Untersuchung an MnZn-Ferriten fest, dass sich Korngrenzen ohne Zwischenphase durch eine hohe Symmetrie bzw. Koinzidenz der benachbarten Körner auszeichnen. Gekrümmte Korngrenzen sind nach Ansicht der Autoren energetisch sehr ungünstig und werden häufig durch die Bildung von Facetten verhindert ${ }^{[i]}$. Hierdurch lässt sich das Auftreten ungünstiger Ladungszustände über die Korngrenze hinweg und insbesondere die direkte Benachbarung gleichnamiger Ladungen verhindern. Intergranulare Phasen sind in der Lage, diese ungünstigen Ladungszustände zu neutralisieren und führen zum Auftreten stärker gekrümmter und damit instabilerer Korngrenzen. Dies illustriert Abbildung 5.4 im Vergleich zwischen einer Korngrenze im diskontinuierlich gewachsenen Gefüge und der Korngrenzenstruktur im normalgewachsenen Gefüge bei einer mit 0,04 Gew.\% $\mathrm{Bi}_{2} \mathrm{O}_{3}$ dotierten Probe.

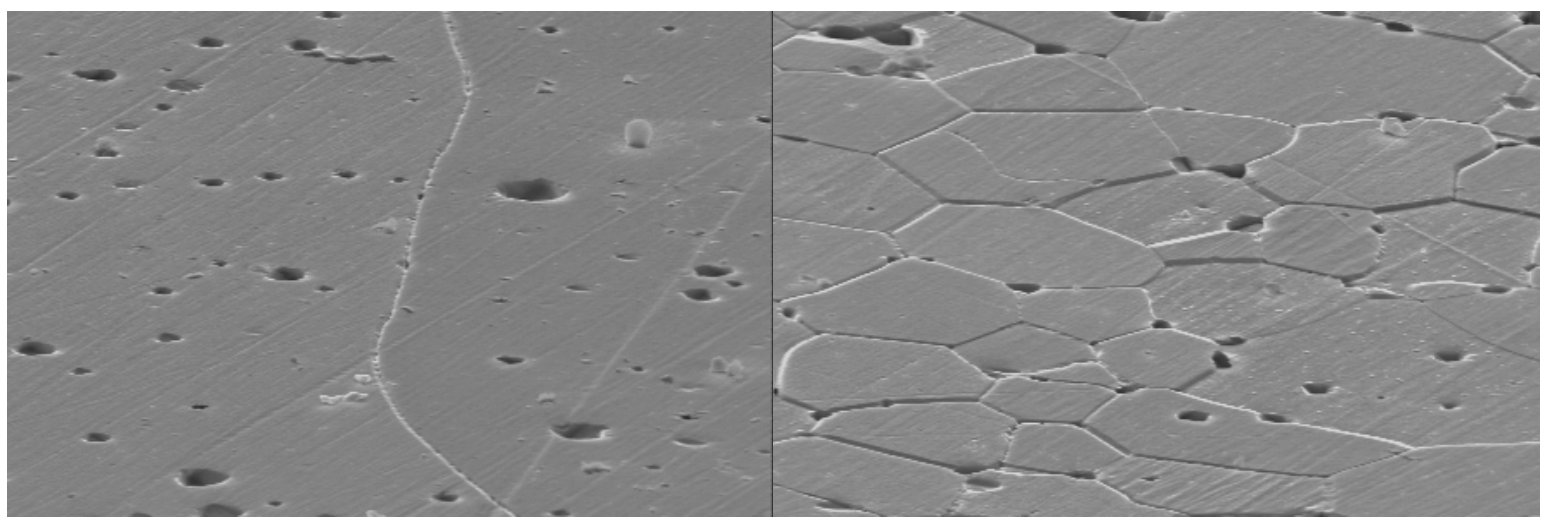

a)

$20 \mu \mathrm{m}$

b)

Abbildung 5.4 Verlauf von Korngrenzen a) zwischen zwei diskontinuierlich gewachsenen Körnern im Inneren und b) im normalgewachsenen Gefüge am Rand der mit 0,04 Gew. $\% \mathrm{Bi}_{2} \mathrm{O}_{3}$ dotierten Probe.

Bei den mit Molybdänoxid dotierten Proben tritt diskontinuierliches Kornwachstum in deutlich geringerem Maße auf. Die Tendenz hierzu zeigt sich insbesondere bei den mit 0,02 Gew.\% dotierten Proben und nimmt bei höheren Konzentrationen wieder ab.

[i] Facettierte Korngrenzen wurden in dieser Arbeit auch bei den undotierten Proben nicht gefunden. Dies kann an ihrem seltenen Vorkommen liegen. Zudem haben alle Proben einen verhältnismäßig hohen Gehalt an Fremdatomen wie Kalzium und Silizium. Fremdatome können durch Segregation in der Korngrenze die Korngrenzenenergie absenken und eine Facettierung verhindern. 
Hierzu passt, dass Nomura und Ochiai Molybdäntrioxid zusammen mit Wolfram, Natrium und Kalium in die Stoffgruppe einordnen, die bei Ferriten überstarkes Kornwachstum unterdrückt [36]. Auch zeigen die Untersuchungen von Jain et al. [72], dass größere Mengen (0,5 Gew.\%) an Molybdänoxid zu einer Abnahme des Kornwachstums führen können (vgl. Abschnitt 2.2.4).

Im Allgemeinen ist mit dem Entstehen einer flüssigen Phase nicht immer ein verbessertes Kornwachstum verbunden. Vielmehr kann es bei größeren Konzentrationen aufgrund der verlängerten Diffusionswege in der interkristallinen Phase auch zu einer Verringerung des Kornwachstums kommen. Des Weiteren lösen sich wahrscheinlich geringe Anteile an Molybdänionen im Kristallgitter und verringern über den Solute-Drag-Mechanismus die Korngrenzenbeweglichkeit. Auch bei Wismutoxiddotierung wird gelegentlich eine Abnahme der Korngröße bei höheren Gehalten beobachtet. So finden Drofenik et al. [70] bei Gehalten $\geq 0,3$ Gew. $\% \mathrm{Bi}_{2} \mathrm{O}_{3}$ ein wieder feiner werdendes Gefüge.

Insgesamt geht das Entstehen von diskontinuierlichem Kornwachstum mit einer hohen intrakristallinen Porosität und einer verringerten Enddichte einher. Die elektrischen Eigenschaften verschlechtern sich zum einen aufgrund der geringeren Dichte (geringeres magnetisches Volumen, innere Scherung), zum anderen führen wahrscheinlich komplexe dolchförmige Bereichsstrukturen und Blochwand-Pinning-Effekte im Inneren der Körner $\mathrm{zu}$ einer Verringerung der Blochwandbeweglichkeit und $\mathrm{zu}$ einer Abnahme der Permeabilität (vgl. Abschnitt 2.1.5, Abbildung 2.6).

\section{Vergleich von theoretischen Ansätzen zur Berechnung des kritischen Haltewinkels}

Vergleicht man den Ansatz zur Berechnung des kritischen Haltewinkels von Hsueh et al. [62] mit dem von Svoboda und Riedel [63] für die in Abbildung 4.7 gezeigte PorenKorngrenzen-Konfiguration, mit einem Haltewinkel $\theta \approx 22^{\circ}$ und einem Öffnungswinkel $\psi_{d n}$, der durch das Anätzen bei der Präparation eher verkleinert wird und mit $\psi_{d n} \geq 84^{\circ}$ abgeschätzt werden kann (vgl. Abschnitt 4.1.2), so findet sich ein Widerspruch zu den Berechnungen von Hsueh et al.. Die Autoren geben für eine solche Konfiguration einen kritischen Haltewinkel $\theta_{k r i t} \leq 16^{\circ}$ an und sagen somit ein Loslösen der Pore aus der Korngrenze voraus. Die Analyse von Svoboda und Riedel ergibt hingegen einen kritischen Haltewinkel $\theta_{k r i t} \approx 37^{\circ}$ und bestätigt die beobachtete Bewegung der Pore mit der Korngrenze. 


\subsubsection{Porenkoaleszenz}

Bei der Verwendung von Molybdäntrioxid als Additiv findet man bei Dotierungen $\geq 0,02$ Gew.\% das Entstehen von Porenagglomeraten. Sie bilden sich zum einen während des Aufheizens im Schwindungsintensivstadium durch lokale Umordnung einzelner Körner oder Kornagglomerate (vgl. Abschnitt 2.2.1). Wie in Abschnitt 2.2.3 beschrieben, kann dieser Effekt durch die Anwesenheit einer Schmelze begünstigt werden. Weiterhin können hohe, ungleichmäßig wirkende Kapillarkräfte, die zu einem Zeitpunkt auftreten, an dem der Formkörper noch über unzureichende Festigkeit verfügt, die Bildung von sehr großen Poren bzw. Porenagglomeraten erleichtern. Ungleichmäßige Kapillarkräfte entstehen z.B. durch Dichteunterschiede im Grünkörper oder durch eine inhomogene Verteilung des schmelzfließenden Additivs. Das Zwischenstadium des Sinterns setzt ein, ohne dass sich ausreichend stabile Kontaktstellen gebildet haben.

Zum anderen können Poren durch Diffusion entlang der Korngrenze anwachsen und sich zu großen Poren agglomerieren [102]. Schatt [38] spricht in diesem Zusammenhang auch vom Prozess des inneren Sinterns.

Wahrscheinlich treten beide Mechanismen auf. Abbildung $4.13 \mathrm{~b}$ ) gibt einen starken Hinweis auf Porenkoaleszenz durch Korngrenzendiffusion. Die größeren Poren, wie sie in Abbildung 4.12 e) gezeigt werden, können evtl. auch schon im Schwindungsintensivstadium entstanden sein. Einmal entstanden, führen die Agglomerate aufgrund des großen Porendurchmessers $R$ nach Gleichung $2.22\left(\bar{p}=A 2 \gamma_{P} \Theta / \bar{R}\right)$ zu einer Verringerung des Kapillardruckes und bauen sich auch bei längerem Sintern nicht ab. 


\subsection{Elektro - Mikrowellen - Hybridsintern}

\subsubsection{Der Verdichtungsprozess}

Bei der Diskussion des Verdichtungsprozesses ist zuerst der Einfluss der Aufheizgeschwindigkeit zu beachten. Ungeachtet des Anteils an Mikrowellenenergie tritt bei ihrer Erhöhung von ca. $5 \mathrm{~K} / \mathrm{min}$ auf $25 \mathrm{~K} / \mathrm{min}$ bei den untersuchten NiZn-Ferriten eine Zunahme der Dichte von 4,8 g/ $\mathrm{cm}^{3}$ auf ca. $5,2 \mathrm{~g} / \mathrm{cm}^{3}$ auf.

Offensichtlich kommt es $\mathrm{zu}$ der in Abschnitt 2.2.1 beschriebenen schnellen nichtisothermen Verdichtung. Das entstandene Gefüge ist sehr feinkristallin, zeichnet sich durch eine geringe Korngrenzenmobilität aus und ist stabil gegen diskontinuierliches Kornwachstum. Im Vergleich hierzu haben die konventionell gesinterte Proben eine hohe intraktristalline Porosität und ein verhältnismäßig grobkristallines Gefüge. Auch wenn die Neigung zu diskontinuierlichem Kornwachstum deutlich geringer ist als bei den $\mathrm{Bi}_{2} \mathrm{O}_{3}$ - und $\mathrm{MoO}_{3}$-dotierten Proben aus Abschnitt 5.1, so stellen die vorhandenen Poren bei den konventionell gesinterten Proben doch kein starkes Hindernis für die sich bewegende Korngrenze dar.

Bei den MnZn-Leistungsferriten lässt sich diese Dichteerhöhung nicht beobachten. Ursache sind höchstwahrscheinlich die schon am Anfang des Kapitels diskutierten, komplexen, diffusionsgesteuerten Phasenbildungsvorgänge, die bei den kalzinierten NiZn-Ferriten nicht stattfinden.

Bei genauerer Betrachtung erkennt man, dass auch der Anteil an Mikrowellenleistung einen Einfluss auf die Enddichte hat (Abbildung 4.25). Hier zeigt sich ein Effekt, der wahrscheinlich auf die gleichmäßigere Erwärmung des Sintergutes bei kombinierter Mikrowellen - Widerstandsbeheizung zurückzuführen ist und in Abschnitt 5.2.4 weiter diskutiert wird. Bei $25 \%$ Mikrowellenanteil an der zugeführten Energie findet sich mit $5,24 \mathrm{~g} / \mathrm{cm}^{3}$ ein Maximum in der Enddichte von $97 \%$ der theoretischen Dichte (vgl. Abbildung 4.25). Durch die Ofenkonstruktion bedingt konnte der der Bereich von 0 $25 \%$ Mikrowellenleistung nicht untersucht werden. Bei der mit reiner Widerstandsbeheizung gesinterten Probe wurde allerdings eine vergleichsweise geringe Dichte von nur 5,19 $\mathrm{g} / \mathrm{cm}^{3}$ gefunden. Dies lässt vermuten, dass das Dichtemaximum tatsächlich in der Nähe von $25 \%$ Mikrowellenleistung erreicht ist. 


\subsubsection{Kornwachstum und Defektstruktur}

Abbildung 4.26 und vgl. Abbildung 4.27 zeigen den Einfluss der Mikrowellenerwärmung auf die Korngröße mit einem Minimum bei $25 \%$ Mikrowellenleistung, also gerade bei den Proben mit maximaler Dichte. Die Entstehung der unterschiedlichen Korngrößen im Sinterprozess ist nur schwer erklärbar und es ist fraglich, ob eine homogenere Erwärmung der Proben für diesen Effekt verantwortlich ist. Dagegen spricht, dass bei den ohne Mikrowellenenergie gesinterten Proben keine Gefügeunterschiede zwischen Randbereich und Korninnerem auftreten. Zudem erfolgt der Temperaturausgleich innerhalb der Proben relativ schnell. Die dafür maßgebliche thermische Relaxationszeit $\tilde{t}$ lässt sich aus den Koeffizienten der Wärmeleitungsgleichung (2.40) und der Wandstärke der Probe $d$ abschätzen und beträgt bei Raumtemperatur für einen Ausgleich zwischen Kern und Rand der Probe ca. 0,6 s:

$$
\widetilde{t}=\frac{c_{w} \rho}{\lambda_{w}} \frac{d^{2}}{4} \approx 0,6 \mathrm{~s}
$$

$\left(c_{w} \approx 10^{3} \mathrm{~J} /(\mathrm{kg} \mathrm{K})\right.$ für $\mathrm{T} \approx 500-900^{\circ} \mathrm{C}[103], \rho \approx 5 \cdot 10^{3} \mathrm{~kg} / \mathrm{m}^{3}, \lambda_{w} \approx 4 \mathrm{~W} /(\mathrm{K} \mathrm{m})$ bei $23^{\circ} \mathrm{C}$ [104], $\left.d \approx 1,4 \cdot 10^{-3} \mathrm{~m}\right)$. Bei reiner Widerstandsbeheizung mit einer Aufheizgeschwindigkeit von $25 \mathrm{~K} / \mathrm{min}$ ergibt sich daraus eine Temperaturdifferenz von $0,3 \mathrm{~K}$. Selbst wenn man annimmt, dass sich die Wärmeleitfähigkeit $\lambda_{w}$ bei höheren Temperaturen um eine Größenordnung verringert, verbleibt eine Temperaturdifferenz von wenigen Kelvin. Eine wesentliche Verlangsamung des Aufheizvorganges im Probeninneren tritt also bei den gewählten Aufheizraten auch bei konventioneller Erwärmung nicht auf und kann die beobachteten Unterschiede in der Korngröße nicht erklären.

An dieser Stelle sind weitere Untersuchungen zum Kornwachstums- und Verdichtungsprozess, sowie zur Wechselwirkung zwischen Mikrowellenstrahlung und der Defektstruktur, notwendig. Von besonderem Interesse ist hierbei der Einfluss der für das schnelle nichtisotherme Sintern wichtigen Versetzungen (vgl. Abschnitt 2.2.1).

Schlüssiger ist hingegen die in Abbildung 4.27 gezeigte Abnahme der Sinterenddichte mit zunehmender Korngröße. Sie setzt voraus, dass die in den Korngrenzen vorliegenden Strukturstörungen zwar im Sinterprozess als Leerstellensenken dienen, aber nicht wesentlich zu einer Verringerung der Dichte des gesinterten Körpers beitragen, was aufgrund der durchgeführten TEM-Untersuchungen (Abschnitt 4.2.4) plausibel erscheint. 
Folglich können sich Poren im feinkristallinen Gefüge besser abbauen. Dieses Verhalten ist experimentell vielfach belegt und passt gleichzeitig $\mathrm{zu}$ den durch Coble [42] am Tetrakaidekaedermodell vorgenommenen Berechnungen (vgl. die Gleichungen 2.19 und 2.20).

\subsubsection{Anfangspermeabilität}

Die Anfangspermeabilität der Proben hängt bei gleicher Zusammensetzung von der Mikrostruktur ab. Die bekannten Untersuchungen und theoretischen Ansätze zur Beschreibung des Zusammenhanges zwischen Korngröße und Anfangspermeabilität (vgl. Abschnitt 2.1.7) finden immer ein Abfallen der Anfangspermeabilität bei kleiner werdender Korngrößen. Allerdings muss der Einfluss der Porosität zumindest im Vergleich zwischen langsam erwärmten, konventionell hergestellten und den hybridgesinterten NiZn-Ferritproben beachtet werden. Insbesondere intrakristalline Poren können wie erwähnt, über Blochwand-Pinning-Effekte und die sich ausbildenden dolchförmigen Domänen, die Permeabilität deutlich verringern. Es zeigt sich nämlich, dass mit einem dichten, feinkristallinen Gefüge ähnliche oder höhere Permeabilitäten wie mit einer verhältnismäßig grobkristallinen, jedoch poröseren Struktur erreichbar sind (vgl. Abbildung 4.24 und Tabelle 4.7). Bei den mit einem Mikrowellenanteil von $30 \%$ gesinterten Proben wurden Permeabilitäten von ca. 1800 gemessen. Dies entspricht einem Anstieg von ungefähr $20 \%$ gegenüber den konventionell gesinterten Proben, bei ansonsten gleichbleibenden oder verbesserten magnetischen Eigenschaften. Offensichtlich lässt sich der Einfluss des feinkristallinen Gefüges durch eine dichtere Mikrostruktur mit einem geringeren Anteil an intrakristallinen Poren kompensieren. Die an den hybridgesinterten Proben durchgeführten TEM-Untersuchungen zeigen zwar das auftreten vereinzelter interkristalliner Phasen, mit einer Breiten von ca. $2 \mathrm{~nm}$, aber ansonsten von Poren und anderen Defekten freie Körner und Korngrenzen.

Gleichzeitig ließ sich die Sinterdauer deutlich reduzieren. Berücksichtigt man eine zusätzliche Entbinderungszeit bei den hybridgesinterten Proben, so ergibt sich eine Verkürzung um ca. $67 \%$ von $1060 \mathrm{~min}$ auf ca. $350 \mathrm{~min}$.

\section{Besonderheiten beim Hybridsintern}

Betrachtet man die mit unterschiedlichem Anteil an Mikrowelle gesinterten Proben und vergleicht den Einfluss von Dichte und Korngröße auf die Anfangspermeabilität, so findet sich das in Abbildung 5.5 gezeigte Verhalten. 


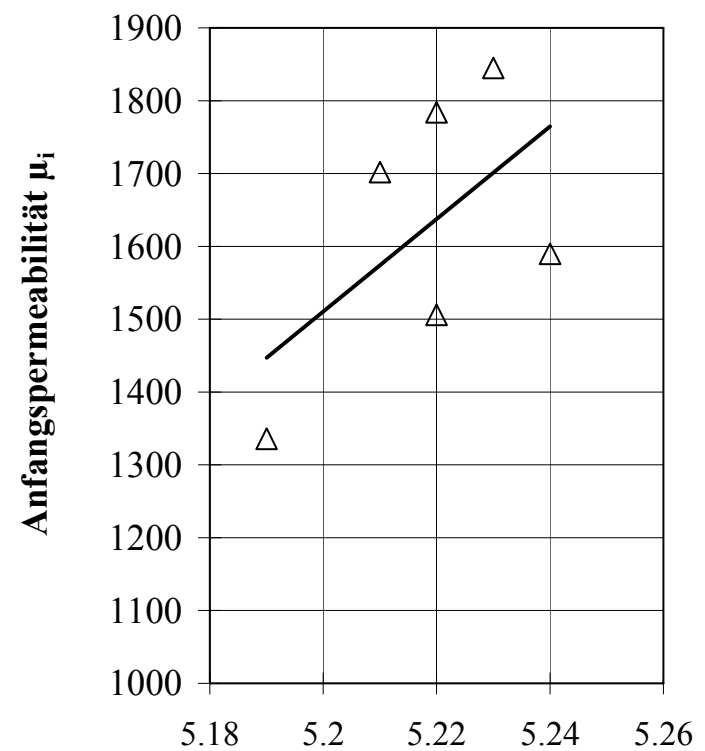

Dichte $\left[\mathrm{g} / \mathrm{cm}^{3}\right]$

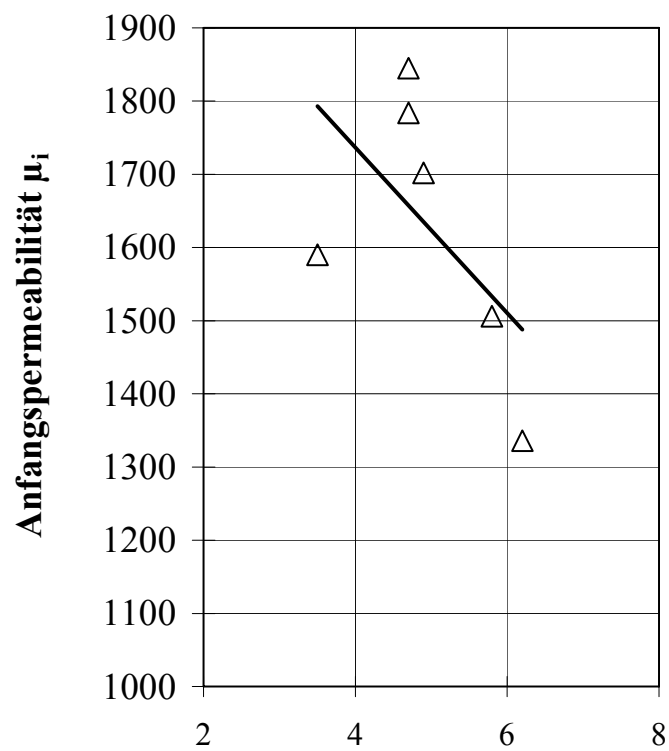

Korngröße $[\mu \mathrm{m}]$

Abbildung 5.5 Zunahme der Anfangspermeabilität mit der Probendichte und mit kleiner werdender Korngröße für NiZn-Ferrite, die mit unterschiedlichem Anteil an Mikrowellenenergie gesintert wurden (vgl. Abbildung 4.27).

Die Anfangspermeabilität nimmt erwartungsgemäß mit der Dichte zu. Ähnlich wie schon in Abschnitt 5.1.2 bei den mit Molybdäntrioxid dotierten Proben erörtert, ist auch hier die Zunahme nicht an ein Anwachsen der mittleren Korngröße gebunden. Vielmehr wächst die Permeabilität trotz kleiner werdender mittlerer Korngröße. Das für kleine Korngrößen erfolgreich eingesetzte NMGB-Modell von Johnson und Visser [21, 23, 25, 28] beschreibt den Zusammenhang zwischen Permeabilität und Korngröße gemäß

$$
\mu_{e}=\frac{\mu_{i} \bar{X}}{\mu_{i} b+\bar{X}}
$$

$\left(\mu_{e}=\right.$ effektive Anfangspermeabilität, $\mu_{i}=$ Anfangspermeabilität im Korninneren, $b=$ effektive Breite der unmagnetischen Korngrenze, $\bar{X}=$ mittlerer Korndurchmesser). Das beobachtete Verhalten kann im Rahmen dieses Modells entweder durch ein Anwachsen der Permeabilität im Korninneren oder durch eine Verringerung der effektiven Korngrenzenbreite $b$ erklärt werden. Beides ist denkbar, wenn man von einer Abnahme der magnetoelastischen Energie aufgrund verringerter mechanischer Spannungen ausgeht. Im korngrenznahen Bereich kann dies die effektive Breite der entmagnetisierten Korngrenze reduzieren, im Korninneren steigt die Permeabilität gemäß Gleichung (2.9).. Offen bleibt, wie sich die Domänenstruktur bei den dicht gesinterten Proben verändert. Neutronenstreuexperimente an NiZn-Ferriten [24] beschreiben den Übergang von 
interkristallinen Blochwänden zu Eindomänenkörnern bei Korngrößen von ca. $3 \mu \mathrm{m}$ (vgl. Abschnitt 2.1.5) und lassen somit ein Gemisch aus zweidomänigen und eindomänigen Körnern erwarten. Denkbar ist allerdings auch, dass sich bei einer Verringerung der effektive Breite der entmagnetisierten Korngrenze über die Korngrenzen hinwegreichende magnetische Domänen ausbilden.

\subsubsection{Der optimale Anteil an Mikrowellenleistung}

Die höchsten Dichten treten bei den feinkristallineren Proben auf (Abbildung 4.27). Diese Proben wurden gerade mit dem optimalen Anteil an Mikrowellenleistung (25\%-30\% der Gesamtleistung) gesintert und besitzen eine ganze Reihe wünschenswerter Eigenschaften.

Dies sind die

- höchsten Sinterenddichten,

- die niedrigste Porosität,

- das feinste Gefüge,

- die höchste Anfangspermeabilität.

Die Kombination dieser Eigenschaften spricht für ein sehr homogenes und spannungsfreies Gefüge.

Wodurch ist der optimale Anteil an Mikrowellenleistung gegeben und welche Mechanismen führen zu den beschriebenen Eigenschaften?

Eine Aufklärung ist aufgrund der Komplexität der Vorgänge äußerst schwierig. Zum einen stellt die Erwärmung einen komplizierten Vorgang dar, bestehend aus Wärmeleitung, Strahlung, Konvektion im Ofenraum und der mit der Temperatur stark zunehmenden Dissipation von Mikrowellenenergie im Ferrit, zum anderen sind die Mechanismen, die bei der schnellen, nichtisothermen Verdichtung wirken, nur unzureichend erforscht.

Es muss davon ausgegangen werden, dass das gefundene Optimum an Mikrowellenleistung in starker Weise von der Konstruktion des Sinterofens und wegen möglicher Abschirmeffekte auch von der Anordnung des Sintergutes im Ofen abhängt. Wie bereits vermutet, ist es gerade durch ein besonders homogene Erwärmung, bzw. durch ein Minimum im Temperaturgradienten $\vec{\nabla} T$ innerhalb des für die Verdichtung entscheidenden Temperaturbereichs von ca. $900-1200{ }^{\circ} \mathrm{C}$ gegeben. Wahrscheinlich kommt es innerhalb weniger Minuten zu einer sehr schnellen Kompaktierung der Formkörper. 
Die dabei entstehende Korngrenzenkonfiguration ist so stabil, dass nach ihrem Entstehen kein nennenswertes Kornwachstum mehr stattfindet.

\subsubsection{Verlustabsenkung bei Leistungsferriten}

Die vorherigen Bemerkungen beziehen sich weitestgehend auf die an den NiZn-Ferriten durchgeführten Untersuchungen. Bei den MnZn-Ferriten wurden aufgrund der oben erwähnten Unterschiede bei der Phasenbildung weder vergleichbare Dichten noch eine ähnlich hohe Permeabilität erzielt.

Allerdings lässt sich auch hier ein feineres Gefüge und damit einhergehend eine merkliche Absenkung der Ummagnetisierungsverluste erreichen. Wie in Abschnitt 4.2.3 gezeigt, werden vornehmlich die intrakristallinen Wirbelstromverluste reduziert und dadurch das Anwachsen der Hystereseverluste kompensiert. Bei einer Frequenz von $1 \mathrm{MHz}$ und einer Induktion von $50 \mathrm{mT}$ lässt sich die Gesamtverlustleistung um $30 \%$ absenken. 


\section{Zusammenfassung und Ausblick}

Die an hochpermeablen MnZn-Ferriten durchgeführten Untersuchungen belegen das Auftreten einer niedrigviskosen, gut benetzenden Schmelze bei Zugabe geringer Mengen an Wismutoxid und Molybdäntrioxid. Wismutoxid bildet mit dem im MnZn-Ferrit auftretenden Phasengemisch mindestens zwei Eutektika mit deutlich unterschiedlichen Gehalten an Eisen- Mangan- und Zinkoxid und eutektischen Temperaturen zwischen $750{ }^{\circ} \mathrm{C}$ und $800^{\circ} \mathrm{C}$. Hiermit verbunden entstehen bei Temperaturen von $800^{\circ} \mathrm{C}$ zwei geschichtete nicht mischbare flüssige Phasen, wobei der Gehalt von umgerechnet ca. 50 Mol.\% Ferrit in einer der Schichten die von Viting und Golubkova für Nickel- und Kobaltferrit [69] angegebenen Konzentrationen deutlich übersteigt. Es handelt sich hierbei wahrscheinlich um eine Phase oder ein Phasengemisch das mit der von Levin und Roth vermuteten metastabilen $\mathrm{BiFeO}_{3}$ - Phase [99] verwandt ist.

Mittels der transmissionselektronenmikroskopischen Untersuchungen konnte bei den wismutoxiddotierten Proben erstmals die Entstehung sehr breiter, intergranularer Flüssigphasen mit einem hohen Anteil an Kalzium und Silizium nachgewiesen werden. Fremdatome reichern sich in der schmelzfließenden Phase an und reduzieren dadurch die im Gefüge auftretenden Spannungen.

Das Kornwachstum der undotierten Probe lässt sich während der isothermen Sinterphase durch $\bar{X}^{n}-\bar{X}_{0}^{n}=\widetilde{K} \exp \left(-Q / k_{B} T\right) \cdot t_{\text {sinter }}$ mit einem Exponenten $\mathrm{n} \approx 3$ gut beschreiben. Bei Dotierung mit Wismutoxidmengen $\leq 0,03$ Gew.\% ändert sich der Exponent nicht. Die Aktivierungsenergie $Q$ senkt sich jedoch um ca. 0,1 eV, was sich in einer Erhöhung der mittleren Korngröße von ca. $20 \mu \mathrm{m}$ auf $25-30 \mu \mathrm{m}$ widerspiegelt. Gleichzeitig können sich Poren über Korngrenzendiffusion gut abbauen und die Porosität $\Theta$ sinkt von über 2,5 \% auf unter 1,5\%, während sich die Permeabilität von 10000 auf ca. 14000 erhöht.

Mit Molybdäntrioxiddotierung lässt sich die Korngröße sogar auf ca. $45 \mu \mathrm{m}$ erhöhen und Permeabilitäten von ca. 17000 erreichen.

Neben Korngröße und Porosität sind die oben erwähnten Entspannungs- und Reinigungseffekte für das Ansteigen der Anfangspermeabilität verantwortlich. Sie führen zu einer Verschiebung des sekundären Permeabilitätsmaximums (SPM). 
Nachteilig wirken sich hingegen Porenkoaleszenz und die Entstehung von diskontinuierlich wachsenden Körnern mit hoher intragranularer Porosität aus. Verantwortlich für letzteres ist neben der erhöhten Korngrenzenbeweglichkeit eine verstärkte Krümmbarkeit und damit erhöhte Instabilität der Korngrenzen mit intergranularer flüssiger Phase.

Strategien für eine weitere Erhöhung der Anfangspermeabilität liegen zum einen in einer verbesserten Verdichtung durch Abbau interkristalliner Poren aufgrund verstärkter Korngrenzendiffusion. Zum anderen muss die Entstehung diskontinuierlich wachsender Körner während des Sintervorgangs unterbunden werden. Dies kann durch eine Verringerung des Anteils an flüssiger Phase oder durch den Einsatz von Additiven geschehen, welche durch Segregation in den Korngrenzen deren Beweglichkeit reduzieren.

Des Weiteren kommt der Vermeidung von Gefügespannungen eine große Bedeutung zu. Gefügespannungen können durch im Kristallgitter gelöste Fremdatome, durch in der Korngrenze segregierte Ausscheidungen und in den Körnern auftretende Inhomogenitäten und Gradienten verursacht werden.

Um Porenkoaleszenz zu vermeiden, ist das Erreichen eines ausreichend stabilen und homogen verdichteten Sinterkörpers vor dem Auftreten der Schmelze von Bedeutung. Hierbei spielen die Temperaturführung beim Erwärmen sowie die Morphologie des Grünkörpers eine wichtige Rolle. Die Untersuchungen zum Mikrowellen-Hybridsintern legen nahe, dass sich über einen schnellen Aufheizprozess eine homogene Verdichtung und eine schnelle Verringerung der Porosität erreichen lässt.

Ein weiterer Punkt ist es, das Verdampfen der flüssigen Phase beim Sintern zu vermeiden. Dies geschieht am einfachsten durch eine effektive Kapselung des Sintergutes. Hierdurch ließen sich in Folge dieser Untersuchungen Ferrite mit Anfangspermeabilitäten von über 20000 herstellen [105 - 107].

Ein zweiter Weg zum Erreichen einer hohen Enddichte und eines defektfreien Gefüges wurde mit der erstmaligen Anwendung der Mikrowellen-Hybridsintertechnik bei weichmagnetischen Ferriten beschritten.

Unabhängig vom Anteil an Mikrowellenleistung kommt es durch eine Erhöhung der Aufheizrate von $5 \mathrm{~K} / \mathrm{min}$ auf $25 \mathrm{~K} / \mathrm{min}$ bei NiZn-Ferriten zu einem deutlichen Anstieg 
der Enddichte von ca. $4,8 \mathrm{~g} / \mathrm{cm}^{3}$ auf $5,2 \mathrm{~g} / \mathrm{cm}^{3}$ durch schnelle, nichtisotherme Verdichtung. Allerdings wird bei MnZn-Ferriten dieser Vorgang durch die während des Aufheizens stattfindenden diffusionsgesteuerten Phasenumwandlungsprozesse unterbunden.

Diese störenden Prozesse können voraussichtlich verhindert werden, wenn sowohl die Kalzination als auch der Aufheizprozess unter Stickstoffatmosphäre durchgeführt wird. Man verwendet dazu symmetrische Sinterkurven und regelt den Sauerstoffpartialdruck auch beim Erwärmen entsprechend den in Gleichung 3.1 beschriebenen Blankschen Gleichgewichtsbedingungen.

Weitere Untersuchungen belegen einen Einfluss der Mikrowellenleistung auf die Enddichte. Bei einem optimalen Anteil von ungefähr $25 \%$ Mikrowellenleistung wurde bei NiZn-Ferriten die höchste Enddichte von $5,24 \mathrm{~g} / \mathrm{cm}^{3}$ oder ca. $97 \%$ der theoretischen Dichte erreicht. Die so hergestellten Proben zeigen die niedrigste Porosität, das feinste Gefüge und die höchste Anfangspermeabilität. Nach Erreichen der Sintertemperatur findet kein nennenswertes Kornwachstum mehr statt.

Die beobachtete Kombination von hoher Permeabilität mit einem feinkristallinen Gefüge ist außergewöhnlich und lässt sich im Rahmen des NMGB-Modelles nur verstehen, wenn man von einer deutlichen Abnahme innerer Spannungen ausgeht. Wahrscheinlich kommt es bei optimierter Mikrowellenleistung zu einer besonders gleichmäßigen Erwärmung im Schwindungsintensivstadium bei Temperaturen von ca. $900-1200{ }^{\circ} \mathrm{C}$.

Aufgrund der hohen Aufheizraten und der trotz des feinkörnigen Gefüges erreichten hohen Permeabilitäten ließ sich die Dauer der isothermen Haltephase reduzieren und der Sinterzyklus um $65 \%$ verkürzen.

Trotz der geringeren Enddichte konnten bei MnZn-Ferriten mit Aufheizraten von 25 $\mathrm{K} / \mathrm{min}$ und einem Mikrowellenanteil von $30 \%$ Permeabilitäten von ca. 1000 erzielt werden. Im Vergleich zur konventionellen Sinterung sind die Wirbelstromverluste durch das feinere Gefüge deutlich reduziert.

Strategien zur weiteren Absenkung der Verluste beinhalten neben einer Verfeinerung des Gefüges, eine Optimierung der intergranularen Phasen und damit einhergehend eine verbesserte Korngrenzenisolation und eine Reduzierung der interkristallinen Wirbelströme. Daneben ist aber auch die Anhebung des spezifischen Widerstandes im Korninneren und die Verringerung von intrakristallinen Wirbelströmen erfolgversprechend. Letzteres kann sowohl durch eine Reduktion des $\mathrm{Fe}^{2+}$-Gehaltes, als auch 
durch eine Verringerung bzw. Substitution der Mn-Ionen erreicht werden. Möglicherweise lassen sich auch NiZn-Ferrite mit niedriger magnetokristalliner Anisotropie für Leistungsanwendungen einsetzen.

Ziel der Maßnahmen ist die Erhöhung der oberen Einsatzfrequenz für die Leistungsübertragung bei Ferriten von derzeit $1 \mathrm{MHz}$ auf ca. $5 \mathrm{MHz}$.

Insgesamt belegen die Untersuchungen zum Mikrowellen-Hybridsintern das Potential einer schnellen Verdichtung für die Herstellung von Ferriten. Neben einer Verkürzung der Sinterzyklen kommt es $\mathrm{zu}$ einer merklichen Verbesserung der magnetischen Eigenschaften.

Wahrscheinlich haben die so hergestellten Materialien aufgrund der feineren Gefüge auch eine verbesserte Bruchfestigkeit und mechanische Stabilität. Diesbezügliche Untersuchungen sind in Vorbereitung.

Möglichkeiten ergeben sich auch für die Herstellung hoch- und mittelpermeabler MnZnFerrite. Mittlere Permeabilitäten von bis zu 8000 lassen sich voraussichtlich schon mit sehr kurzen Sinterzyklen aufgrund des zwar feinen, aber spannungs- und porenfreien Gefüges erreichen. Hohe und höchste Permeabilitäten von über 30000 kann man erwarten, wenn es gelingt, durch kontrolliertes Kornwachstum und den Einsatz niedrigschmelzender Additive ein gleichmäßiges Gefüge mit mittleren Korngrößen von ca. $50 \mu \mathrm{m}$ auszubilden. 


\section{Symbol- und Abkürzungsverzeichnis}

\begin{tabular}{|c|c|c|}
\hline Symbol & Einheit & Bezeichnung \\
\hline$\alpha_{h}$ & $W s / m^{3}$ & Verlustfaktor der Hystereseverluste \\
\hline$\alpha_{w}$ & $W s^{2} / m^{3}$ & Verlustfaktor der Wirbelstromverluste \\
\hline$\gamma$ & $J / m^{2}$ & Oberflächenenergie \\
\hline$\gamma_{B}$ & $J / m^{2}$ & Oberflächenenergie der Korngrenze \\
\hline$\gamma_{B W}$ & $J / m^{2}$ & Blochwandenergie \\
\hline$\gamma_{P}$ & $J / m^{2}$ & Oberflächenenergie einer Porenoberfläche \\
\hline$\underline{\gamma}$ & & materialabhängige Ausbreitungskonstante $\underline{\gamma}=\sqrt{\underline{\mu} \underline{\varepsilon}}$ \\
\hline$\delta, \delta_{x}$ & & Verlustwinkel $(x=\varepsilon, \mu, \gamma)$ \\
\hline$\varepsilon_{0}$ & $A s / V m$ & Elektrische Feldkonstante $\varepsilon_{0}=8,85410^{-12} \mathrm{As} / \mathrm{Vm}$ \\
\hline$\underline{\varepsilon}, \varepsilon^{\prime}, \varepsilon^{\prime \prime}$ & & komplexe Dielektrizitätszahl, ihr Real- und Imaginärteil \\
\hline$\varepsilon$ & & Schwindung $\quad \Delta L / L_{0}$ teilw. in $\%$ \\
\hline$\dot{\varepsilon}$ & $1 / s$ & Schwindungsgeschwindigkeit \\
\hline$\theta$ & $\circ$ & Haltewinkel zwischen Pore und Korngrenze \\
\hline$\Theta$ & & Porosität (Volumenanteil, teilw. in \%) \\
\hline$\Theta_{B}$ & $1 / m^{2}$ & mittlere Porendichte in der Korngrenze \\
\hline$\kappa$ & $1 / m$ & mittlere Oberflächenkrümmung \\
\hline$\lambda$ & & Magnetostriktionskonstante \\
\hline$\lambda_{w}$ & $J /(K s m)$ & Wärmeleitfähigkeit \\
\hline$\mu_{0}$ & $V s / A m$ & Magnetische Feldkonstante $\mu_{0}=4 \pi 10^{-7} \mathrm{Vs} / \mathrm{Am}$ \\
\hline$\mu_{a}$ & & Amplitudenpermeabilität \\
\hline$\mu_{e}$ & & effektive Anfangspermeabilität \\
\hline$\mu_{i}$ & & Anfangspermeabilität \\
\hline$\mu_{B}$ & $A m^{2}$ & Bohrsches Magneton $\mu_{B}=9,27410^{-24} \mathrm{Am}^{2}$ \\
\hline$\underline{\mu}, \mu^{\prime}, \mu^{\prime \prime}$ & & komplexe Permeabilität, ihr Real- und Imaginärteil \\
\hline$\rho$ & $\Omega m$ & Spezifischer Widerstand \\
\hline$\rho$ & $m$ & Oberflächenradius \\
\hline$\sigma$ & $1 / \Omega m$ & Leitfähigkeit \\
\hline$\sigma$ & $N / m^{2}$ & mechanische Spannung \\
\hline$\psi_{x}, \bar{\psi}_{x}$ & $\circ$ & (mittlerer) Öffnungswinkel, $x$ indiziert die Lage (n, dn) \\
\hline$\omega$ & $1 / s$ & Kreisfrequenz $\omega=2 \pi f$ \\
\hline$\Omega$ & $m^{3}$ & Atomvolumen oder Leerstellenvolumen \\
\hline$A^{\prime}$ & $J / m$ & effektive Austauschkonstante \\
\hline$A_{\varepsilon}$ & $m^{2}$ & Effektiver magnetischer Querschnitt \\
\hline$b$ & $m$ & (wirksame) Breite der Korngrenze \\
\hline$B$ & $T=V S / m^{2}$ & Magnetische Induktion \\
\hline$\hat{B}$ & $T$ & Spitzenwert der magnetischen Induktion \\
\hline$B_{r}$ & $T$ & Remanenzinduktion \\
\hline
\end{tabular}




\begin{tabular}{|c|c|c|}
\hline Symbol & Einheit & Bezeichnung \\
\hline$B_{\text {sat }}$ & $T$ & Sättigungsinduktion \\
\hline$c$ & $\mathrm{~m} / \mathrm{s}$ & Lichtgeschwindigkeit $c=2,99810^{8} \mathrm{~m} / \mathrm{s}$ \\
\hline$c_{1}$ & & Blanksche Gleichgewichtskonstante \\
\hline$c_{2}$ & $K$ & Blanksche Gleichgewichtskonstante \\
\hline$c_{w}$ & $J /(K \mathrm{~kg})$ & Wärmekapazität \\
\hline$C_{V}$ & & Leerstellenkonzentration $C_{V}=N_{V} / N_{\text {Ion }}$ \\
\hline$C_{V 0}$ & & Leerstellengleichgewichtskonzentration $C_{V 0}=N_{V 0} / N_{I o n}$ \\
\hline$C_{V k a t}$ & & Kationenleerstellenkonzentration $\quad C_{\text {Vkat }}=N_{\text {Vkat }} / N_{\text {kat }}$ \\
\hline$d$ & $m$ & Blochwandbreite \\
\hline$D$ & $m^{2} / s$ & Diffusionskonstante \\
\hline$D_{V}$ & $m^{2} / s$ & bei Volumenselbstdiffusion \\
\hline$D_{B}$ & $m^{2} / s$ & bei Korngrenzendiffusion \\
\hline$D_{S}$ & $m^{2} / s$ & bei Oberflächendiffusion \\
\hline$D_{F}$ & & Desakkommodationsbeiwert \\
\hline$E$ & $J$ & Energie \\
\hline$E_{K}$ & $J$ & Magnetokristalline Anisotropieenergie \\
\hline$E_{U}$ & $J$ & bei einachsiger induzierter Anisotropie \\
\hline$E_{M E}$ & $J$ & Magnetoelastische Energie \\
\hline$E, \hat{E}$ & $V / m$ & Elektrische Feldstärke \\
\hline$f$ & $1 / s$ & Frequenz \\
\hline$F_{P}$ & $N$ & Haltekraft, die eine Pore auf eine Korngrenze ausübt \\
\hline$\hbar$ & $J_{S}$ & Drehimpulsquantum $\hbar=h /(2 \pi)$ mit $h=6,626210^{-34} J_{S}$ \\
\hline$H$ & $A / m$ & Magnetische Feldstärke \\
\hline$\hat{H}$ & $A / m$ & Spitzenwert der magnetischen Feldstärke \\
\hline$H_{c}$ & $A / m$ & Koerzitivfeldstärke \\
\hline$K_{1}, K_{2}$ & $J / m^{3}$ & Konstanten der Kristallanisotropie \\
\hline$K_{U}$ & $J / m^{3}$ & Konstante der einachsigen induzierten Anisotropie \\
\hline$\widetilde{K}, \tilde{k}$ & $m^{n} / s$ & Konstanten des Kornwachstums $(\mathrm{n}=2 \ldots 6)$ \\
\hline$k, \underline{k}$ & $1 / m$ & Wellenzahl \\
\hline$k_{B}$ & $J / K$ & Boltzmannkonstante $k_{B}=1,380510^{-23} \mathrm{~J} / \mathrm{K}$ \\
\hline$L$ & $H=V S / A$ & Induktivität \\
\hline$L$ & $\mathrm{Nm}$ & Bahndrehmoment \\
\hline$l_{e}$ & $m$ & Effektive magnetische Weglänge \\
\hline$M_{B}$ & $m^{3} /(N s)$ & Korngrenzenbeweglichkeit \\
\hline$M_{P}$ & $m /(N s)$ & Porenbeweglichkeit \\
\hline$M_{S}, M_{A}, M_{B}$ & $A / m$ & $\begin{array}{l}\text { Sättigungsmagnetisierung, tw. bezogen auf die Untergitter } \\
\text { A und B. }\end{array}$ \\
\hline$n$ & & Exponent der Kornwachstumsgleichung $n=2 \ldots 6$ \\
\hline$N$ & & Windungszahl \\
\hline $\bar{N}_{V}$ & $1 / m^{3}$ & Versetzungsdichte \\
\hline$P_{\text {diss }}, P_{S}, P$ & $W=J / s$ & Verlustleistung \\
\hline
\end{tabular}




\begin{tabular}{c|c|l}
\hline Symbol & Einheit & Bezeichnung \\
\hline$P_{w .} P_{h}$ & $W / m^{3}$ & Wirbelstromverluste, Hystereseverluste \\
$p, \bar{p}$ & $N / m^{2}$ & Druck, Kapillardruck \\
$Q$ & $J$ & Energie, Aktivierungsenergie \\
$Q$ & & Güte \\
$R$ & $\Omega$ & Wirkwiderstand \\
$R$ & $m$ & Porenradius \\
$S$ & $N m$ & Spindrehmoment \\
$\tilde{t}$ & $S$ & thermische Relaxationszeit \\
$t_{\text {sinter }}$ & $h$ & isotherme Sinterzeit \\
$T$ & $K$ & Temperatur \\
$T_{c}$ & $K$ & Curietemperatur \\
$v_{B}, v_{P}$ & $m / s$ & Korngrenzen- bzw. Porengeschwindigkeit \\
$V, V_{e}$ & $m^{3}$ & Volumen bzw. effektives magnetisches Volumen \\
$X, \bar{X}$ & $m$ & Korngröße (Korndurchmesser), mittlerer Korngröße \\
$Z, \underline{Z}$ & $\Omega$ & Impedanz $\underline{Z}=R+i \omega L$ \\
\hline
\end{tabular}




\section{Literaturverzeichnis}

[1] L. Néel, Thèse, Strasbourg, 1931

[2] S. Hilpert, Ber. Dtsch. Chem. Ges., 42 (1909), S. 2248

[3] S. Hilpert, R. Schweinhagen, Z. Phys. Chem., (B)31 (1935), S. 1

[4] Y. Tako, T. Takei, Japan Patent Nr. 98.844, (1932)

[5] M. Guyot, Science of Ferrite in the $20^{\text {th }}$ and $21^{\text {st }}$ Centuries, Proc. ICF 8, Kyoto, The Japan Society of Powder and Powder Metallurgy (2000), S. XXV

[6] E.J.W. Verwey, E.L. Heilmann, J. Chem. Phys. 15 (1947), S. 174

[7] L. Néel, Propriétés Magnétiques des Ferrites: Ferrimagnétisme et Antiferromagnétisme, Les Annales de Physique (1948)

[8] J.L. Snoek, New developments in Ferromagnetic Materials, Physica, 3 (1936), S. 463

[9] J.L. Snoek, New Developments in Ferromagnetic Materials, Elsevier Ed., Amsterdam, 1949, S. 68-98

[10] J. Smit, H.P.J. Wijn, Ferrites, Philips Technical Library, Eindhoven, 1959

[11] S. Chikazumi, Physics of Magnetism, John Wiley \& Sons, New York, 1964

[12] S.Krupicka, Physik der Ferrite und der verwandten magnetischen Oxide, Vieweg \& Sohn, Braunschweig, 1973

[13] L. Michalowsky, Ferritwerkstoffe, Akademie-Verlag, Berlin, 1985

[14] A. Goldman, Modern Ferrite Technology, Van Nostrand Reinhold, New York, 1990

[15] J.C. Slonczewski, Origin of Magnetic Anisotropy in Cobalt-Substituted Magnetite, J. Appl. Phys., 30 (1959), S. 310 S

[16] J. Dreikorn, Anfangspermeabilität und Frequenzstabilität von Mn-Zn-Ferriten mit oxidischen Additiven, Diplomarbeit, Universität Göttingen, 1995

[17] P. Beyer, Hermsdorfer Techn. Mitteilungen, 12 (1972), S. 1059

[18] V.N. Shambalev, L.A. Bashkirov, V.V. Pankov, Self-Diffusion of Cations in the Solid Solution System of Ti-Substituted Mn-Zn Ferrites, Phys. Stat. Sol. (a), 112 (1989), S. K71 
[19] J.-H. Lee, M. Martin, H.-I. Yoo, Self- and impurity cation diffusion in manganese zinc ferrite, J. Physics and Chemistry of Solids, 61 (2000), S. 1597 - 605

[20] R .Freer, Self-diffusion and impurity diffusion in oxides, J. Materials Science, 15 (1980), S. 803-24

[21] P.J. van der Zaag, et al., The initial permeability of polycrystalline MnZn ferrites: The influence of domain and microstructure, J. Appl. Phys., 74 [6] (1993), S. 4085-95

[22] J. Aarts, I. Abu Shiekah, P.J. van der Zaag, Domain structure in polycrystalline MnZn ferrite imaged by magnetic force microscopy, J. Appl. Phys., 85 [10] (1999), S. 7302-9

[23] M.T. Johnson et al., Magnetic permeability and intragranulare domain structure in polycrystalline ferrites, J. Magn. Magn. Mat., 104-107 (1992), S. 421-2

[24] P.J. van der Zaag, P.J. van der Valk, M.Th. Rekveldt, A domain size effect in the magnetic hysteresis of NiZn-ferrites, Appl. Phys. Lett., 69 [19] (1996), S. 2927-9

[25] P.J. van der Zaag, M. Kohlenbrander, M.Th. Rekveldt, The effect of intragranulare domain walls in MgMnZn-ferrite, J.Appl. Phys., 83 [11], S. 6870-2

[26] L. Néel, Cahiers Phys., 25 (1944), S. 21

[27] A. Globus, Some physical considerations about domain wall size theory of magnetization mechanisms, J. Phys. 38, Suppl. C1, (1977) S. 1-15

[28] M.T. Johnson, E.G. Visser, A coherent model for the complex permeability in polycrystalline ferrites, IEEE Trans. Magn. MAG-26 [5] (1990) S. 1987-9

[29] E. Roess, I. Hanke, Die Mikrostruktur hochpermeabler Ferrite, Z. Angew.Phys., 29 [4] (1970), S. 225

[30] C.G. Koops, On the Dispersion of Resistivity and Dielectric Constant of some Semiconductors at Audiofrequencies, Phys. Rev., 83 [1] (1951), S. 121-4

[31] P.F. Bongers, F.J.A. den Broeder, J.P.M. Damen, Defects, Grain Boundary Segregation and Secondary Phases of Ferrites in Relation to the Magnetic Properties, Proc. ICF 3, Kyoto, Japan (1980) 
[32] K. Yasuhara, K. Takagawa, The Effect of Cooling Conditions in Sintering Process..., Proc. ICF 8, Kyoto, Jap. Soc. of Powder and Powder Metallurgy (2000), S. 393-5

[33] T. Kuroda, H. Saita et al., Microstructure and Magnetic Properties of MnZn Ferrites in relation to Behavior of Ca in Sintering Process, ebd. S. 506-8

[34] M.Drofenik, A.Znidarsic, D.Makovec, Ca Redistribution in MnZn Ferrite Grain Boundaries during Heat Treatment in a Reducing Atmosphere, ebd., S.530 - 2

[35] R. Mauczok, V.T. Zaspalis, Binder burnout-material-process interaction during sintering of MnZn-ferrites, J. of the European Ceramic Society, 20 [12] (2000), S. 2121

[36] T. Nomura, T. Ochiai, Current topics in the field of materials technology of soft ferrites, Proc. Magnetic Ceramic Symp., Indianapolis, Am. Ceram. Soc., (1994), S. $211-26$

[37] J.E. Geguzin, Physik des Sinterns, Dt. Verlag für Grundstoffindustrie, Leipzig, 1973

[38] W. Schatt, Sintervorgänge, VDI-Verlag, Düsseldorf, 1992

[39] R.L. Coble, Sintering Crystalline Solids I. Intermediate and Final Stage Sintering Models J. Appl. Phys., 32 (1961), S. 787-92

[40] L. Michalowsky (Hrsg.), Neue keramische Werkstoffe, Dt. Verlag für Grundstoffindustrie, Leipzig, 1994

[41] J.S. Reed, Introduction to the principles of ceramic processing, John Wiley \& Sons, New York, 1988

[42] N.J. Shaw, Densification and coarsening during solid state sintering of ceramics [...], Powder Metallurgy International, 21 (1989), S. 6

[43] F. Wakai et al., Superplasticity: Deformation Mechanisms and Microstructures, Materials Characterization, 37 [5] (1996); S. 331-41

[44] I.W. Chen, L.A. Xue, Development of Superplastic Structural Ceramics, J. Am. Ceram. Soc., 73 [9] (1990), S. 2585-2609

[45] W. Schatt, B. Vetter, E. Friedrich, Non-isothermal shrinkage of compacts, Powder Metallurgy, 34 [3] (1991), S. 179-82 
[46] J.H. She, T. Ohji, Rapid densification of alumina ceramics with fine grains and high strength, J. Mat. Sci. Lett., 21 [18] (2002), S. 1405-6

[47] N. Murayama, W. Shin, Effect of rapid heating on densification and grain growth in hot pressed alumina, J. Ceram. Soc. Jpn., 108 [9] (2000), S. 799 - 802

[48] L. Minyoung-Lee, M.P. Borom, Rapid rate sintering of $\mathrm{Al}_{2} \mathrm{O}_{3}$-TiC composites for cutting tool applications, Adv. Ceram. Mater., 3 [1] (1988), S. 38-44

[49] M. Paulus, Cinétique de croissance normale des cristaux dans les ferrites polycrystallins, Phys. Stat. Sol., 2 (1962), S. 1181

[50] R.J. Brook, The Impurity Drag Effect and Grain Growth Kinetics, Scr. Metall., 2 [7] (1968), S. $355-78$

[51] K.W. Lay, Grain Growth in $\mathrm{UO}_{2}-\mathrm{Al}_{2} \mathrm{O}_{3}$ in the Presence of a liquid Phase, J. Am. Ceram. Soc., 51 [7] (1968), S. 373-6

[52] S.I. Nunes, R.C. Brandt, Grain Growth of $\mathrm{ZnO}-\mathrm{Bi}_{2} \mathrm{O}_{3}$ Ceramics with $\mathrm{Al}_{2} \mathrm{O}_{3}$ Additions, J. Am. Ceram. Soc., 78 [9] (1995), S. 2469-75

[53] Y. Huang, F.J. Humphreys, Measurements of Grain Boundary Mobility During Recrystallization [...], Acta Metall. 47 [7] (1999), S. 2259-68

[54] A.D. Rollett, G. Gottstein, L.S. Shvindlerman, D.A. Molodov, Grain boundary mobility - a brief review, Z. Metallkd., 95 [4] (2004), S. 226-9

[55] A. Kazaryan, B.R. Patton, S.A. Dregia, Y. Wang, On the the Theory of Grain Growth in Systems with Anisotropic Boundary Mobility, Acta mater., 50 (2002), S. $499-510$

[56] M. Upmanyu, D.J. Srolovitz, L.S. Shvindlerman, G. Gottstein, Misorientation Dependence of Intrinsic Grain Boundary Mobility [...], Acta mater., 47 [14] (1999), S. 3901-14

[57] E. Brosh, R.Z. Shneck, Effect of Strong Anisotropy in Grain-Boundary Energy on Boundary Mobility in Abnormally Grown Grains, J. Am. Ceram. Soc., 87 [4] (2004), S. 640-3

[58] P.E. Zovas, R.M. German, Retarded Grain Boundary Mobility in Activated Sintered Molybdenum, Metallurgical Transactions, 15A (1984), S. 1103-10 
[59] A.H. Heuer, The role of $\mathrm{MgO}$ in the sintering of alumina, J. Am. Ceram. Soc., 62 [5-6] (1979), S. 317-8

[60] A.H. Heuer, Reply to:Comments to „The role of $\mathrm{MgO}$ in the sintering of alumina “ J. Am. Ceram. Soc., 63 [3-4] (1980), S. 230-1

[61] M.-P. Harmer, R.J. Brook, The effect of $\mathrm{MgO}$ additions on the kinetics of hot pressing in $\mathrm{Al}_{2} \mathrm{O}_{3}$, J. Mat. Sci., 15 (1980), S. 3017-24

[62] C.H. Hsueh, A.G. Evans, R.L. Coble, Microstructure development during final /intermediate Stage - I. Pore / Grain Boundary Separation, Acta metall., 30 [7] (1982), S. 1269-79

[63] J. Svoboda, H. Riedel, Pore-Boundary Interaction and Evolution Equations for the Porosity and the Grain size During Sintering, Acta metall. mater., 40 [11] (1992), S. 2829-40

[64] R.M. German, Liquid Phase Sintering, Plenum Press, New York, 1985

[65] H.-H. Park, S.-J. Cho, D.N. Yoon, Pore Filling Process in Liquid Phase Sintering, Metall. Trans., 15A (1984), S. 1085-90

[66] J.-H. Choi, N.-M. Hwang, D.-Y. Kim, Pore-Boundary Behaviour During Sintering of Pure and $\mathrm{Bi}_{2} \mathrm{O}_{3}$-Doped Ceramics, J. Am. Ceram- Soc., 84 [6] (2001), S. $1398-400$

[67] T. Senda, R.C. Bradt, Grain growth in sintered $\mathrm{ZnO}$ and $\mathrm{ZnO}-\mathrm{Bi}_{2} \mathrm{O}_{3}$ ceramics J. Am. Ceram. Soc., 73, [1] (1990), S. 106-14

[68] J. Kim,T. Kimura; T.Yamaguchi, Effect of bismuth oxide content on the sintering of zinc oxide, J. Am. Ceram. Soc., 72 [8] (1989), S. 1541-4

[69] L.M. Viting, G.P. Golubkova, R.S. Roth (Ed.) Phase Diagrams for Ceramists 4, Fig. 5402 und Fig. 5422, Am. Ceram. Soc. Columbus (Ohio), 1981

[70] M. Drofenik, A. Žnidaršič, D. Makovec, Influence of the addition of $\mathrm{Bi}_{2} \mathrm{O}_{3}$ on the Grain Growth and magnetic Permeability of MnZn Ferrites, J. Am. Ceram. Soc., 81 [11] (1998), S. 2841-8

[71] A. Matsukawa (TDK Corp.), Europäisches Patent, EP 0628524 A1, (1994) 
[72] G.C. Jain, B.K. Das, N.C.Goel, Effect of $\mathrm{MoO}_{3}$ Addition on the Grain Growth Kinetics of a Manganese Zinc Ferite, J. Am. Ceram. Soc., 62 [1-2] (1979), S. 7985

[73] Y. Matsuo, K. Ono, M. Ishikura, Effects of $\mathrm{MoO}_{3}$ Addition on Manganese Zinc Ferrites, IEEE Trans. on Magnetics 33 [5] (1997), S. 3751-3

[74] K. Ono, S. Fukunaga, Y. Matsuo, MnZn-Ferrites with $\mathrm{MoO}_{3}$ and other Additives, IEEE Trans. on Magnetics 35 [5] (1999), S. 3406-8

[75] R.W. Bruce, Microwave Processing of Materials, MRS Symposium Proceedings, 124 (1988), S. 3

[76] W.R. Tinga, Microwave Processing of Materials, MRS Symposium Proceedings, 124 (1988), S. 33

[77] J. Yang, B. Zhang; Z. Xu et al., Neutron diffraction study of Mn-Zn ferrite containing titanium, Chin. J. Phys. USA, A11 (1987), S. 1610-13

[78] C. Michalk, Mössbauer Study of MnZn Ferrite Formation, J Magn. Magn Mat., 68 (1987), S. 157-9

[79] J. Rossel, Mikrostrukturelles Konstruieren zur Gefügeoptimierung von MnZnFerriten [...], Dissertation, Universität Göttingen (1999)

[80] C. Michalk, Festkörperreaktionskinetik bei der Spinellbildung und Oxidation von MnZn-Ferriten, Habilitation, Universität Leipzig (1990)

[81] J.M. Blank, Equilibrium atmosphere schedules for the cooling of ferrites, J. Appl. Phys. Suppl. to 32 (1961), S. 3

[82] R. Morineau, M. Paulus, Chart of $\mathrm{PO}_{2}$ versus temperature and oxidation degree [...], IEEE Trans. Magn., Mag-11 [5] (1975), S. 1312-4

[83] A. Goldman, Modern Ferrite Technology, Van Nostrand Reinhold, NewYork, 1990

[84] Abschlußbericht zum Projekt: AiF.-Nr. 12072 B/2: Mikrowellen-HybridSinterung, (2002)

[85] DIN IEC 62044-3: Cores made of soft magnetic materials - Measuring methods Part 3: Magnetic properties at high excitation level 
[86] DIN EN 60205: Berechnung der effektiven Kernparameter magnetischer Formteile

[87] IWATSU ELECTRIC Co. LTD, Tokyo, Operation Manual SY-8216

[88] H.E.Exner, H.P.Hougardy, Einführung in die quantitative Gefügeanalyse, Deutsche Gesellschaft für Metallkunde, Oberursel, 1986

[89] J. Dreikorn, L. Michalowsky; Influence of $\mathrm{Bi}_{2} \mathrm{O}_{3}$ on microstructure of High Permeability MnZn Ferrites, Proc. ICF 8, Kyoto, The Japan Society of Powder and Powder Metallurgy (2000), S. 554-5

[90] J. Dreikorn. H.C. Freyhardt, L. Michalowsky, Einfluß niedrigviskoser Phasen auf die Gefügebildung hochpermeabler MnZn-Ferrite, Abstract in: Verhandlungen der DPG, Frühjahrstagung, Münster (1999), S.802

[91] K.J. Brook, Controlled Grain Growth. Treatise on Material Science and Technology, 9 (Ed. F.F.Y.Wang), New York, Academic Press, 1976

[92] R.B. Heslop, P.L. Robinson, Inorganic Chemistry, Elsevier, Amsterdam, 1967

[93] W. Kleber nach Ahrens 1952, An Introduction to Crystallography, VEB Verlag Technik, Berlin, 1970, S. 153-5

[94] C.Y. Tay, K.S. Liu I.N. Lin, Improvement on Power Loss Properties of Mn-Zn Ferrites by Microwave Sintering Process, Proc. ICF 8, Kyoto, The Japan Society of Powder and Powder Metallurgy (2000), S. 585-7

[95] J. Dreikorn, M. Buryan, S. Leparoux, H. Krause, T. Lampke, Microwave Hybrid Sintering of Soft Ferrites, Proc. ICF9, San Francisco, The American Ceramic Society (2004), S.15-20

[96] H. Baumgartner, J. Dreikorn, R.Dreyer, L- Michalowsky, E. Pippel, J. Woltersdorf, Manganese-Zinc-Ferrites with improved Magnetic and Mechanical Properties, Proc. ICF 7, Bordeaux, J. Phys. IV France, 7(1996), S. C1-67

[97] A. Nakata, H. Chihara, A. Sasaki, Microscopy study of grain boundary region in polycristalline ferrites, J. Appl- Phys., 57 [1] (1985), S. 4177-9

[98] E. Otsuki, S. Yamada, Analysis of Power Loss in Mn-Zn Ferrites for Switching Power Supplies, Proc. Magnetic Ceramic Symp., Indianapolis, Am. Ceram. Soc., (1994), S. 147-54 
[99] E.M. Levin, R.S. Roth, Polymorphism of Bismuth Sesquioxide. II. Effect of additions on the polymorphism of $\mathrm{Bi}_{2} \mathrm{O}_{3}$ J. Res. Nat. Bur. Stand. - A Phys and Chem., 68A [2] (1964), S. 197-206

[100] T. Kuroda, H. Saita et al., Microstructure and Magnetic Properties of MnZn Ferrites in relation to Behavior of $\mathrm{Ca}$ in Sintering Process, Proc. ICF8, Kyoto, Jap. Soc. of Powder and Powder Metallurgy (2000), S.506-8

[101] J.-Y. Laval, C. Cabanel, M.-H. Berger, Local Electrical Behavior, Crystallography, and Chemistry of Grain Boundaries in Mn-Zn Ferrites, J. Am. Ceram. Soc., 81, [5] (1998), S. 1133-40

[102] M.D. Drory, A.G. Evans, Effects of Voids at Three-Grain Junctions on Sintering and Creep Damage, J. Am. Ceram. Soc., 67, [11] (1984), S. 727-31

[103] N.A. Landiya, V.S. Varazashvili, G.D. Chachanidze, Enthalpie and heat capacity of nickel-zinc ferrites in the range of 25-1000 C, Viniti N185-67dep. (1967), S. 12, Infotherm Datenbank, www.fiz-chemie.de

[104] Y.S. Touloukian (Hrsg), Thermophysical properties of matter, Teil 2: Thermal conductivity, nonmetalic solids, New York, IFI/ Plenum, 1970, S. 298

[105] J. Dreikorn, Manganese-zinc ferrites with improved properties, Magnews, August 1997, S.13

[106] J. Dreikorn et al. Manganese-zinc ferrites with improved properties, Journal de physique / 4, 8 [2] (1998), S. 457-60

[107] KASCHKE KG GmbH \& Co, Göttingen, Materialdatenblatt K 20000 


\section{Danksagungen}

Herrn Prof. Dr. H. C. Freyhardt danke ich herzlich für die Anregung und für die stete Unterstützung und Förderung dieser Arbeit. Seine Ideen und Vorschläge haben viel zum Gelingen beigetragen.

Ein herzliches Dankeschön geht auch an die Kaschke KG GmbH \& Co. und insbesondere an Herrn Prof. Dr. L. Michalowsky für seine engagierte Betreuung und für anregende und weiterführende Gespräche und Vorschläge. Die Zusammenarbeit mit ihm war immer sehr hilfreich und angenehm. Besonders bereichernd war die Möglichkeit, in mehreren Forschungsprojekten auf nationaler und internationaler Ebene mitzuwirken.

Ein besonderes Dankeschön gilt allen Mitarbeiter der Kaschke KG GmbH \& Co, die durch fachliche Anregungen, tatkräftige Hilfe und motivierende Nachfrage zum Erfolg dieser Arbeit beigetragen haben. Insbesondere möchte ich hierbei Herrn Dr. J. Rossel, Herrn Dr. R. Dreyer und die Mitarbeiter der Materialentwicklung, Herrn Dr. U. Sicker, Frau I. Dykta, Frau I. Degener und Frau M. Krebs erwähnen.

Bedanken möchte ich mich auch bei den Mitarbeitern des Instituts für Materialphysik, insbesondere bei Herrn Dr. P.-J. Wilbrandt für die Einarbeitung in die Transmissionselektronenmikroskopie, bei Frau H.-P. Quan für die Hilfe bei der Probenpräparation, sowie bei Herrn D. Plischke für die Unterstützung bei den REM-Aufnahmen.

Ein weiteres Dankeschön geht an die im Rahmen der Forschungsgemeinschaft Industrieofenbau (FOGI) am Forschungsprojekt Mikrowellen-Hybridsintertechnik beteiligten Firmen und Institute, sowie an das BMWi für die finanzielle Unterstützung der Forschung.

Ganz besonders bedanken möchte ich mich bei den mitwirkenden Mitarbeitern des Instituts für Wärmetechnik und Thermodynamik der TU Bergakademie Freiberg unter Führung von Herrn Prof. Dr.-Ing. G. Walter, bei Frau Dr.-Ing. S. Leparoux, Herrn Dr.-Ing. H. Krause, und Herrn Dr.-Ing M. Buryan, für die sehr gute und freundliche Zusammenarbeit und für die am Institut durchgeführten Sinterversuche mit dem Hybridsinterofen. Das gleiche gilt für Herrn Dr.-Ing. T. Lampke vom Institut für Maschinenbau der TU Chemnitz, welcher im Rahmen des Projektes für die mikrostrukturellen Untersuchungen zuständig war. 
Ein Dankeschön geht auch an Herrn Dr. D. Hesse vom MPI für Mikrostrukturphysik in Halle, der im Rahmen des von der Kaschke KG GmbH \& Co. und dem Hermsdorfer Institut für Technische Keramik (HITK) initiierten und vom BMBF geförderten Verbundvorhabens Einsatz von nanodispersen Pulvern zur Herstellung von Mn-Ferriten mit verbesserten Eigenschaften die TEM-Untersuchungen an den hybridgesinterten Ferriten vorgenommen hat.

Abschließend möchte ich mich bei meiner Familie und meinen Freunden bedanken. Diejenigen, die mich während des Erstellens der Arbeit unterstützt, ermuntert und immer wieder motiviert haben. Herzlichen Dank auch an alle, die bei der Durchsicht und Korrektur der Arbeit geholfen haben. 


\section{Lebenslauf}

Am 14. April 1968 wurde ich in Freiburg im Breisgau als Sohn von Werner Dreikorn und seiner Frau Sieglinde (geb. Schütz) geboren. Ich besitze die deutsche Staatsangehörigkeit. Von 1974 - 1978 besuchte ich dort die Tulla-Grundschule, von 1978 - 1984 die WeiherhofRealschule und ab 1984 die Richard-Fehrenbach-Gewerbeschule, ein berufliches Gymnasium technischer Richtung, das ich im Mai 1987 mit der Allgemeinen Hochschulreife verließ.

Vom Februar 1988 bis zum September 1989 leistete ich Wehrersatzdienst in der Kinderheimat-Erlenbach in Fürth-Erlenbach/Odw..

Von Oktober 1989 bis zum September 1991 studierte ich Physik an der Albert-LudwigUniversität in Freiburg im Breisgau. Mit dem Erhalt des Vordiploms im Herbst 1991 wechselte ich an die Technische Universität Chemnitz, wo ich mein Studium bis zum September 1992 fortsetzte. Nach einem erneuten Wechsel an die Georg-AugustUniversität Göttingen wählte ich als Vertiefungsrichtung Material und Festkörperphysik und fertigte ab Januar 1994 am Institut für Metallphysik (heute Institut für Materialphysik) unter Anleitung von Prof. Dr. H. C. Freyhardt in Zusammenarbeit mit der Kaschke KG $\mathrm{GmbH} \&$ Co meine Diplomarbeit mit dem Titel „Anfangspermeabilität und Frequenzstabilität von Mn-Zn-Ferriten mit oxidischen Additiven“ an. Am 23. Juni 1995 erhielt ich mein Diplom des Fachbereichs Physik der Georg - August - Universität Göttingen.

Seit August 1995 bin bei der Kaschke KG GmbH \& Co in der Entwicklungsabteilung für weichmagnetischen Ferrite fest angestellt, seit 1999 leite ich die Abteilung. Zu meiner Tätigkeit im Bereich der Materialentwicklung, gehört auch die Mitarbeit in mehreren Forschungsprojekten, wie dem Verbundvorhaben Exakt II zur Laserbearbeitung von Keramiken (1995-1998), dem EU-Projekt BE-4562 zum Thema MultifrequenzMikrowellensintern von Keramiken (1998-2002) und dem FOGI-Projekt AiF.-Nr. 12072 B/2 zum Mikrowellen-Hybridsintern von Werkstoffen (1999-2001).

Seit Dezember 1998 arbeite ich an meiner von Prof. Dr. H. C. Freyhardt betreuten und von Prof. Dr. L. Michalowsky seitens der Firma angeleiteten externen Dissertation, in die sowohl Ergebnisse aus der innerbetrieblichen Forschung, als auch aus den FOGIForschungsprojekt einflossen. 